\title{
Modeling the oriented strandboard manufacturing process and the oriented strandboard continuous rotary drying system
}

\author{
John R. Noffsinger \\ West Virginia University
}

Follow this and additional works at: https://researchrepository.wvu.edu/etd

\author{
Recommended Citation \\ Noffsinger, John R., "Modeling the oriented strandboard manufacturing process and the oriented \\ strandboard continuous rotary drying system" (2004). Graduate Theses, Dissertations, and Problem \\ Reports. 2104. \\ https://researchrepository.wvu.edu/etd/2104
}

This Dissertation is protected by copyright and/or related rights. It has been brought to you by the The Research Repository @ WVU with permission from the rights-holder(s). You are free to use this Dissertation in any way that is permitted by the copyright and related rights legislation that applies to your use. For other uses you must obtain permission from the rights-holder(s) directly, unless additional rights are indicated by a Creative Commons license in the record and/ or on the work itself. This Dissertation has been accepted for inclusion in WVU Graduate Theses, Dissertations, and Problem Reports collection by an authorized administrator of The Research Repository @ WVU. For more information, please contact researchrepository@mail.wvu.edu. 
Modeling the Oriented Strandboard Manufacturing Process and the Oriented Strandboard Continuous Rotary Drying System

John R. Noffsinger

Dissertation submitted to the

Davis College of Agriculture, Forestry, and Consumer Sciences at West Virginia University

in partial fulfillment of the requirements for the degree of

\author{
Doctor of Philosophy \\ in
}

Forest Resource Science

\author{
R. Bruce Anderson, Ph.D., Chair \\ James P. Armstrong, Ph.D. \\ Benjamin Dawson-Andoh, Ph.D. \\ Dick Kleinknecht, Ph.D. \\ Elemer Lang, Ph.D.
}

Department of Wood Science and Technology

Morgantown, West Virginia

2004

Keywords: Oriented Strandboard, Multivariate Data Analysis, Projection to Latent Structures, Rotary Drying, Multivariate SPC

Copyright 2004 John R. Noffsinger 


\begin{abstract}
Modeling the Oriented Strandboard Manufacturing Process and the Oriented Strandboard Continuous Rotary Drying System
\end{abstract}

John R. Noffsinger

Oriented Strand Board (OSB) is the leading structural panel product used in residential building construction. This dissertation describes three models and a statistical process control technique all designed to aid manufacturers to cost effectively manufacture OSB. The first model is an OSB Mill Process Flow Model that defines the processing steps and the desired outcomes. The second model is an OSB Mill Model, an Excel ${ }^{\circledR}$ based computer program, designed to answer operational "what if" and "tradeoff" questions. The model is a spreadsheet representation of the OSB production process. The third model is an OSB Dryer Model that predicts the dryer outlet moisture content derived using a multivariate data analysis technique called projection to latent structures by means of Partial Least Squares (PLS). PLS was instrumental in identifying outlet temperature and heat source temperatures as the most influential dryer system variables in predicting dryer outlet moisture content. The SPC technique is Multivariate Statistical Process Control (MSPC) that uses multivariate scores or Hotelling $\mathrm{T}^{2}$ to determine the state of the drying process; and if the drying process is out of control, what process variables influenced the process shift. 
To Dr. Terrell and Juanita Noffsinger, my parents, for their encouragement and love

To Karen, my loving wife, for her encouragement, patience, and support

To Nicole, Tasha, and Julianne, my daughters, for bringing joy and happiness into my life and who I love and deeply admire. 


\section{ACKNOWLEDGEMENTS \\ Many Hands Make the Burden Light \\ Latin Proverb}

This work would not be possible without the support, guidance, and help from many. The author would like to express his sincere appreciation to the committee and to Dr. R. Bruce Anderson, the chairman. Dr. Anderson provided his guidance and positive support throughout even when times got "tough". It was helpful to have Dr. Anderson's perspective as a student who also earned his doctorate while working full time as the author has done. Appreciation is also expressed to Dr. Elemer Lang who showed me a different side of teaching - that of a mentor for his students who sincerely cares for them and their success. It goes without saying Dr. Ben Dawson-Andoh helped the author with his enthusiastic attitude toward learning, and if I ever felt down, just "hanging" around Dr. Dawson-Andoh in the hallway would change my point of view. Dr. Jim Armstrong was very supportive of my efforts to work full time for Weyerhaeuser and to pursue my doctorate. I appreciate his openness when I first approached him about undertaking this endeavor. The final member of the committee is Dr. Dick Kleinknecht who works for Weyerhaeuser as an R\&D statistician. It was Dr. Kleinknecht's foresight that allowed the author to attend a conference on Multivariate Data Analysis's Projection to Latent Structures (PLS) by Dr. Svante Wold. Dr. Wold is the author of the analytical technique used in this research. The conference opened up new avenues on how to deal with the masses of data collected in today's forest product manufacturing plants. Dick was very supportive in helping the author understand PLS and was persistent in getting the data collected and analyzed.

The author thanks his employer, Weyerhaeuser Company, for its support both emotionally as well as financially while I worked full time as the Sutton OSB plant technical manager. David Craft, General Manager, was very supportive of my educational endeavors, as was Chris Serbyn, Technical Team Leader who filled in for me at the plant while I was in Morgantown sitting in class or in the library. Kerry Miller, General Manager, who has since replaced David Craft was instrumental in getting me moving again after a year spent on developing a new flooring product. He stated after some discussion on the value of this work, "What do I need to get this model sooner, send 
you home for 30 days?" This helped me to realize the value of the work. Jody Gray and Kenny Shaver who worked to ensure the instruments and devices used in the work were properly calibrated. Brian Wilson, Technical Team Leader, covered for the author while he worked on the data collection and analysis phases of this work and the writing of the dissertation. A-team and specifically Duane Read who ran the dryers when the data were collected and when the model was verified, and Grayg Ralphsnyder who had ideas on how to install devices to make the model more useful.

I have listed numerous people who had a "hand" in this work, but it would not be possible if it were not for Karen, my wife, who has stood by me over the past 33 years and has encouraged me to do whatever I wanted to do. She has been very supportive through this work but also during the last months. I want to acknowledge my parents who encouraged me to do more that I thought I could do and I want to say thank you to my father, Dr. Terrell L. Noffsinger, who instilled in me the real love of learning.

I want to acknowledge my three daughters. Nicole is completing her doctorate in counseling psychology and is at Harvard completing her internship. Tasha is a BS Registered Nurse pursuing her masters as a family nurse practitioner while working full time at Virginia Commonwealth University hospital. Julianne has blessed me with a beautiful granddaughter and grandson who help me see the fun side of life.

I want to acknowledge my Lord Jesus Christ who has provided the Peace and Comfort at times when I struggle with life. For without Him, there is no Peace. 


\section{TABLE OF CONTENTS}

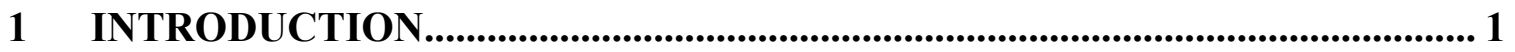

2 OBJECTIVES AND DISSERTATION STRUCTURE ........................................ 6

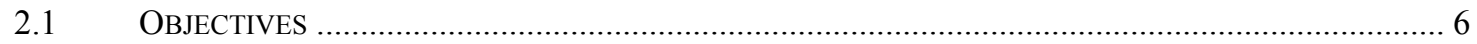

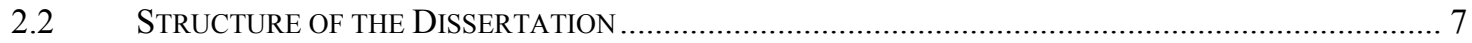

3 LITERATURE REVIEW ...................................................................................... 9

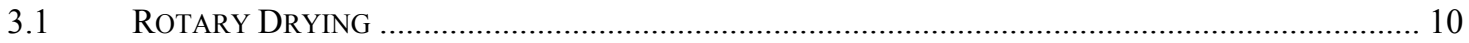

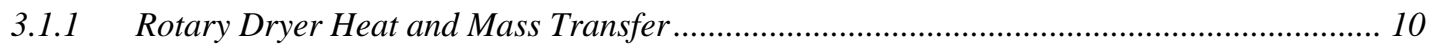

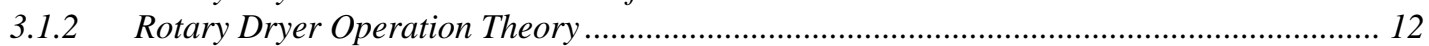

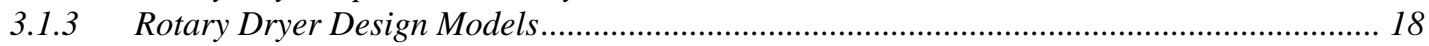

3.1.4 Dryer Variables Effect on Outlet Moisture Content ........................................................... 19

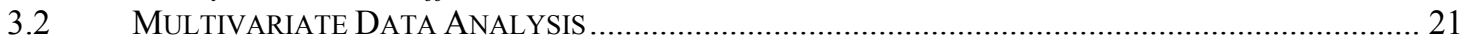

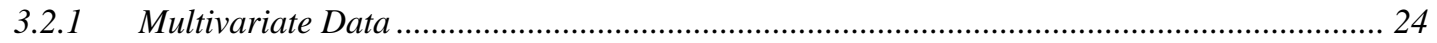

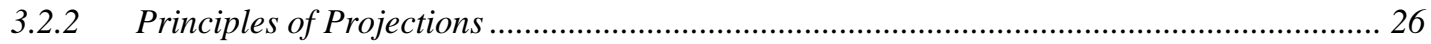

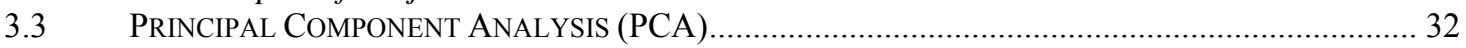

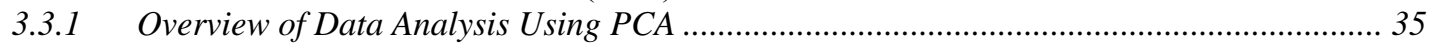

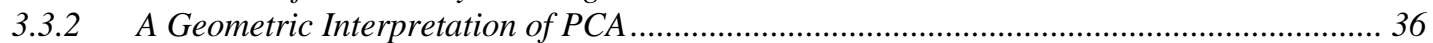

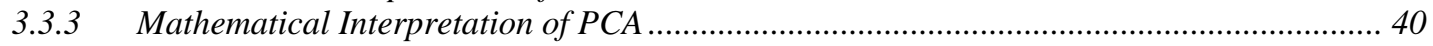

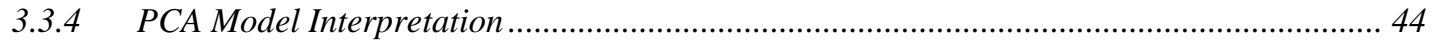

3.4 Projection to Latent Structures By Means of Partial Least SQuare ......................... 47

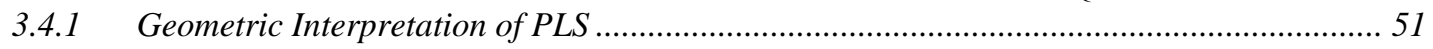

3.4.2 Mathematical Interpretation of PLS - Classical PLS Algorithm.......................................... 58

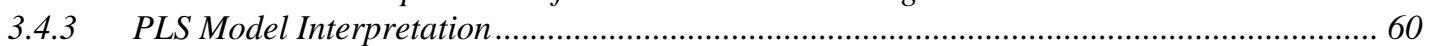

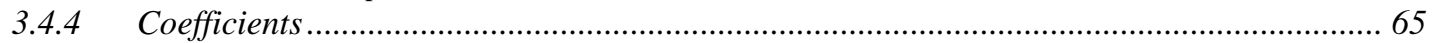

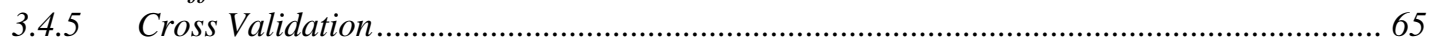

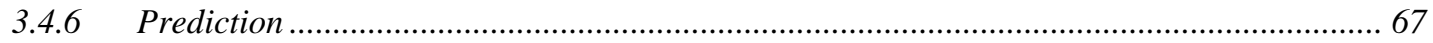

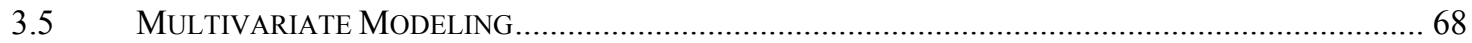

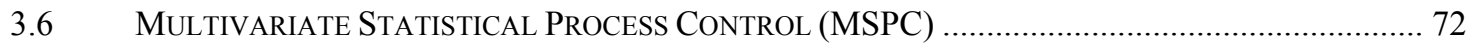

4 METHODS AND MATERIALS ................................................................... 76

4.1 OSB OPERATIONS - OSB PROCESS FLOW AND OSB MILL MODELING....................................... 76

4.2 DRYER HEAT SOURCE AND ROTARY DRYER - DRYER MODELING ………….................................... 77

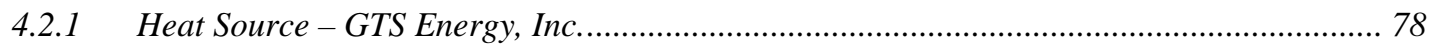

4.2.2 Dryer System - Operation Using Outlet Temperature Control............................................ 83

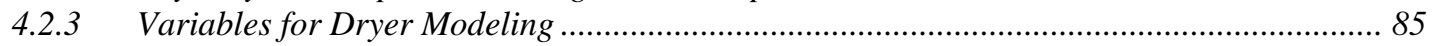

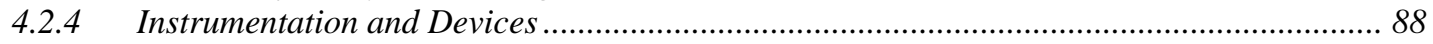

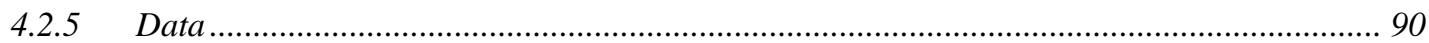

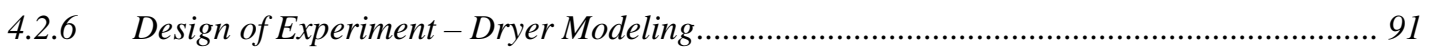

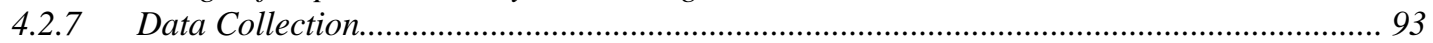

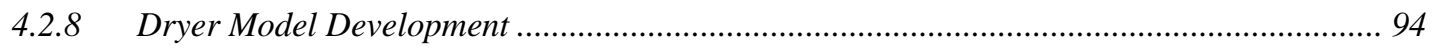

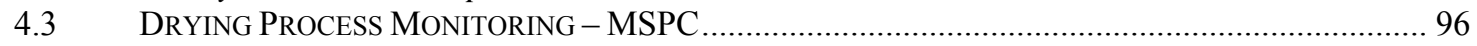

5 THE OSB PROCESS FLOW MODEL ............................................................... 97

5.1 OSB Mill Process Flow - Key Process VARIABLeS ……................................................ 97

6 THE OSB MILL MODEL ..................................................................................... 102

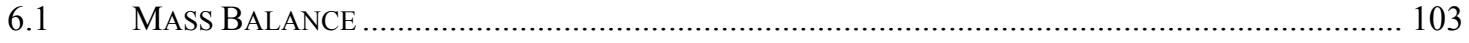

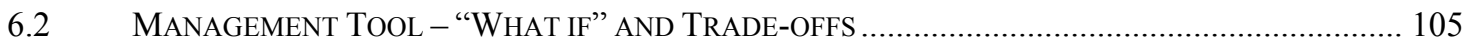

6.2.1 Strander Knife Changes vs. Advance Time..................................................................... 105

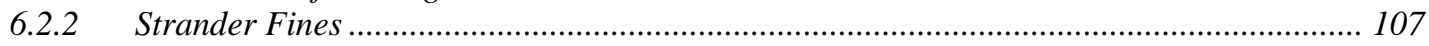

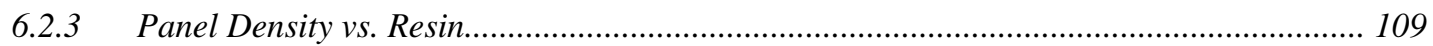


7 DRYING MODEL DEVELOPMENT, VALIDATION, AND PREDICTION111

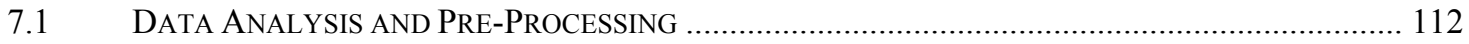

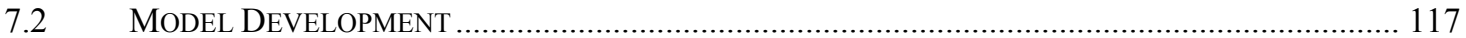

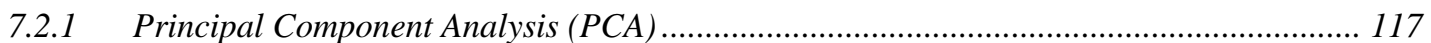

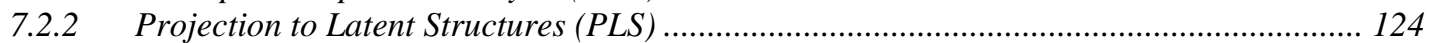

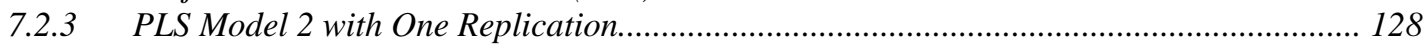

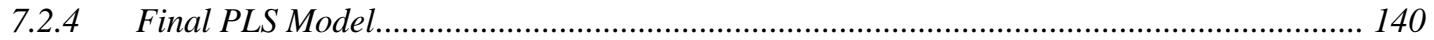

7.2.5 Summary of PLS Model Development ……….................................................................. 153

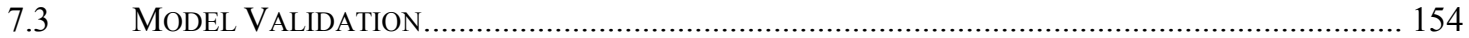

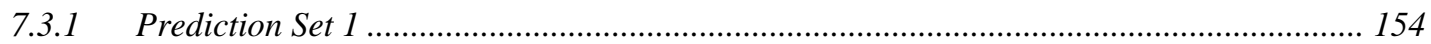

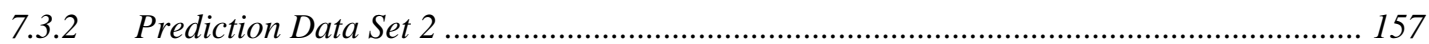

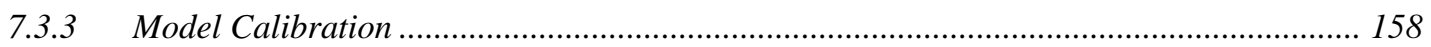

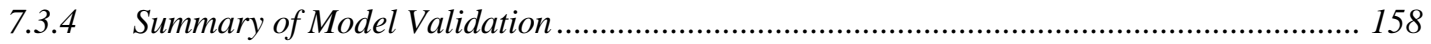

8 MULTIVARIATE STATISTICAL PROCESS CONTROL ............................. 160

8.1 MSPC USING OSB DRYING DATA AND THE PLS DRYER MODEL ........................................... 163

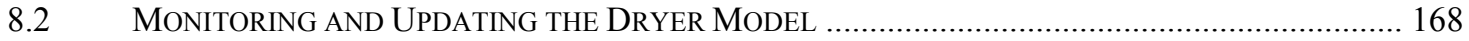

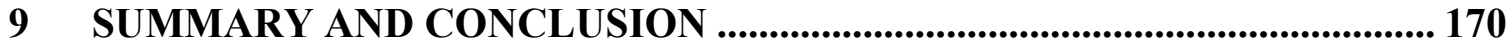

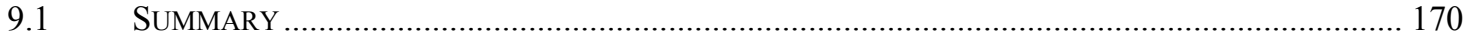

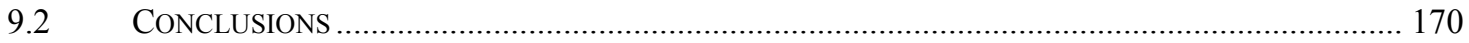

10 RECOMMENDATONS FOR FURTHER RESEARCH ............................. 172

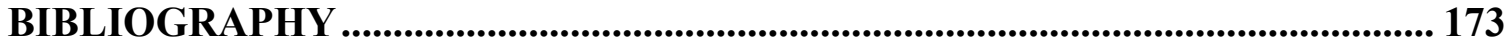

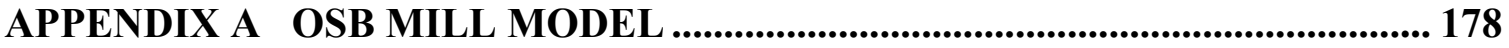

APPENDIX B EFFECT OF AUTOCORRELATED DATA ON COMPOSITE PANEL PRODUCTION MONITORING AND CONTROL: A COMPARISON OF SPC TECHNIQUES (PUBLISHED IN FOR PROD J. 2002. CO-AUTHORED

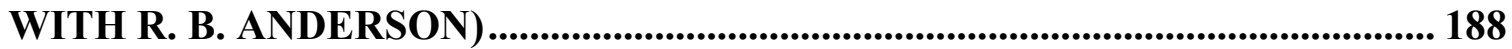

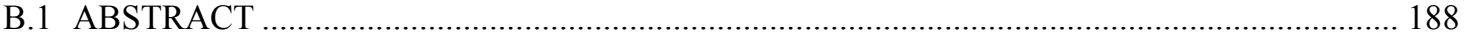

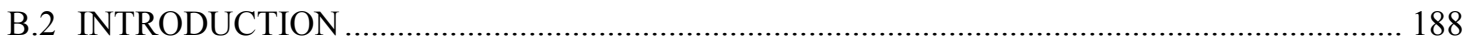

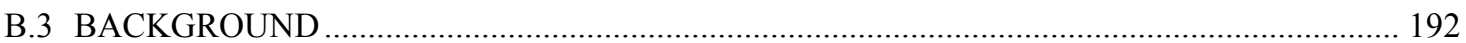

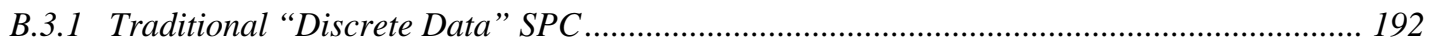

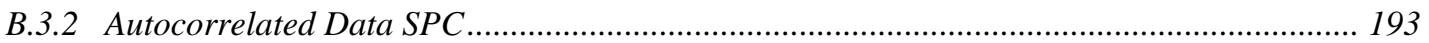

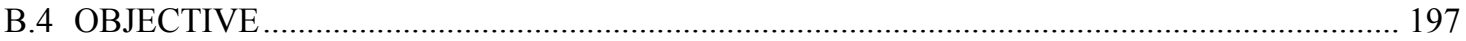

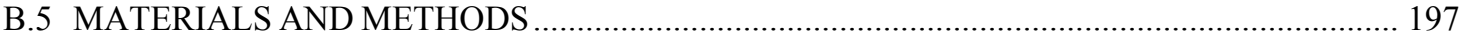

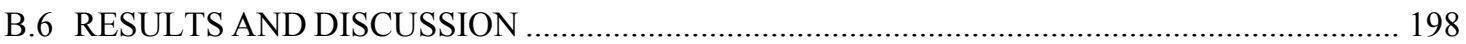

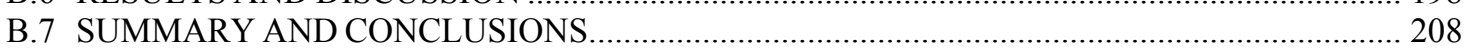

APPENDIX C - ARIMA MODELS.................................................................... 210

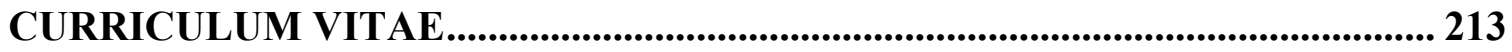




\section{LIST OF TABLES}

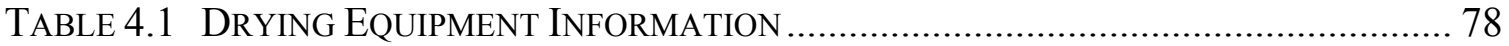

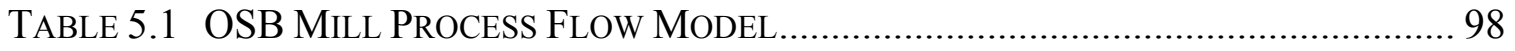

TABLE 6.1 STRANDER FINES VS. RECOVERY WoOd COST AND FINES IN BOARD ........... 109

TABLE 7.1 DRYER MODEL STUDY VARIABLES........................................................... 113

TABLE 7.2 TREATMENTS Run ORder Classes AND SAMPle SizE AFTER THE LAG ...... 114

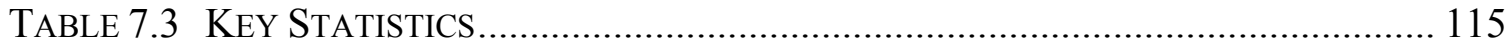

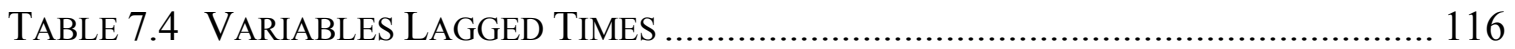

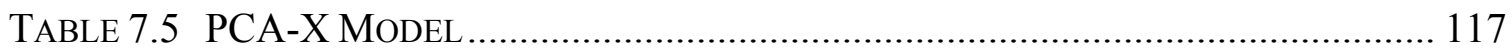

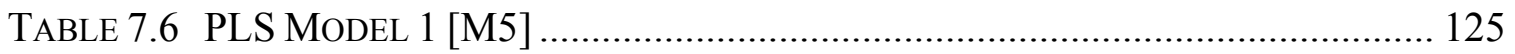

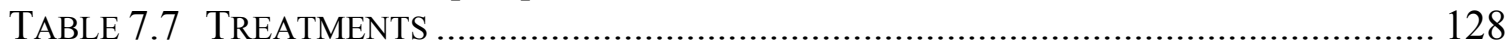

TABLE 7.8 PLS MODEL 2 [M6] ONE REPLICATION OF TREATMENTS ............................. 129

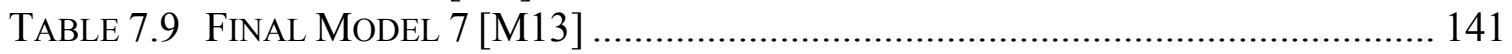

TABLE 7.10 VARIABLE WEIGHTS BY COMPONENT ..................................................... 143

TABle 7.11 Model Coefficients [M13] - Centered and Scaled AS WELl AS RAW

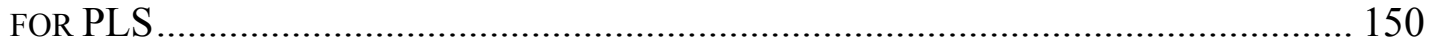

TABLE B.1 SUMMARY OF CONTROL CHARTS INVESTIGATED. ......................................... 203

TABle B.2 Models Used to Approximate the Rotary Dryer Moisture Content

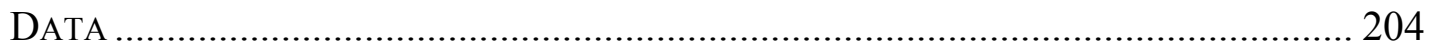




\section{LIST OF FIGURES}

FIGURE 3.1 THE X-Y COORDINATE SYSTEM DEPICTING GRAPHICALLY THE RELATIONSHIP BETWEEN HEIGHT AND WEIGHT.

FIGURE 3.2 THE X-Y-Z COORDINATE SYSTEM DEPICTING GRAPHICALLY THE RELATIONSHIP BETWEEN HEIGHT, WEIGHT, AND AGE................................................................ 28

FIGURE 3.3 THE PLANE IS A WINDOW INTO THE MULTIDIMENSIONAL SPACE. EACH OBSERVATION IS PROJECTED TO THE PLANE IN ORDER TO GET NEW COORDINATE VALUES (SCORES) IN THE NEW COORDINATE SYSTEM (ERIKSSON ET AL. 2001)........... 29

FIGURE 3.4 DATA TABLE OR MATRIX $(15$ X 20) WITH SCORES FOR EACH OBSERVATION AND LOADINGS FOR EACH VARIABLE.

FIGURE 3.5 PCA DERIVES A MODEL THAT FITS THE DATA IN A LEAST SQUARES SENSE. PCA CAN BE UNDERSTOOD AS MAXIMIZING THE VARIANCE OF THE PROJECTION COORDINATES (ERIKSSON ET AL. 2001).

FigURE 3.6 CENTERING DATA IS DEPICTED BY MOVING THE AVERAGE POINT TO THE ORIGIN OF THE GRAPH (ERIKSSON ET AL. 2001).

FIGURE 3.7 THE DIRECTION OF THE PC1 IN RELATION TO THE ORIGINAL VARIABLES IS GIVEN BY THE COSINE OF THE ANGLES $\alpha_{1}, \alpha_{2}$, AND $\alpha_{3}$

Figure 3.8 THE PLS MODEL ( $M=1$ ) IS DEPICTED AS REDUCING THE VARIATION IN THE $Y$ VARIABLE AS THE COMPONENTS ARE DETERMINED IN THE LEFT PORTION OF THE FIGURE THE RESIDUALS F VALUES ARE REDUCED AS THE VARIATION IS REMOVED BY THE COMPONENTS AS SHOWN IN THE RIGHT PORTION OF THE FIGURE (ERIKSSON ET AL. 2001) 52

FIGURE 3.9 PLS MATRICES (UMETRICS, INC. 2003). 57

Figure 4.1 WonderWare Computer Screen of THE Heat Source (GTS Energy,

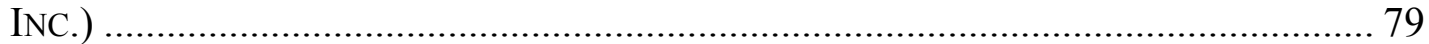

Figure 4.2 WONDERWARE COMPUTER SCREEN OF DRYING SYSTEM ............................ 84

Figure 6.1 MASS BALANCE CHARTS ......................................................................... 104

Figure 6.2 Strander Production Study Knife Changes vs. CARriage Advance TIME AND THEIR AFFECT ON PRODUCTION............................................................. 107

Figure 6.3 Fines vs. Stroke Count ANd EFFECT ON WoOd Cost ............................. 108

Figure 7.1 PCA-X for Dryer Model DeVelopment - Score Plot t[1]/T[2] .......... 118

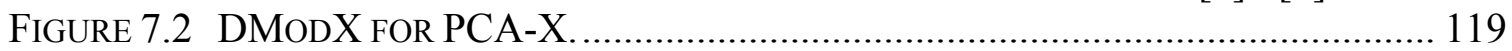

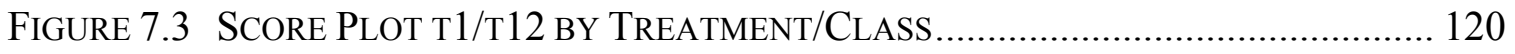

FIGURE 7.4 LOADING PLOT FOR PCA-X P[1]/P[2] ...................................................... 121

FiguRE 7.5 RESIDUALS BY TREATMENT AND Run FOR PLS-MODEL 1 ........................ 126

Figure 7.6 SCORE PlOt For PLS MOdEL WITH ALL RUNS ....................................... 127

Figure 7.7 Residuals By TREATMENT AND Run FOR PLS-MODEL 1 REPLICATION .... 128

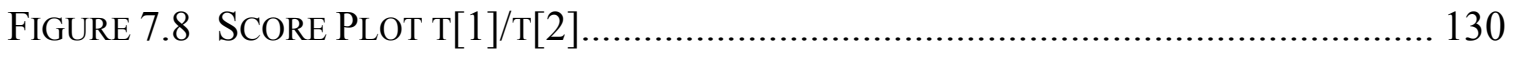

Figure 7.9 PLS-Model 2, t[1] vs. Y [OUTLET Moisture CONTENT] ........................ 131

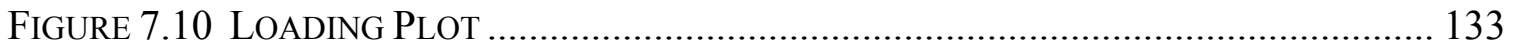

Figure 7.11 Times Series Plot OF the SCORES FOR PLS-Model 2 COMPONENT $1 \ldots 135$

Figure 7.12 Time Series Plot of the Actual Outlet Temperature Data

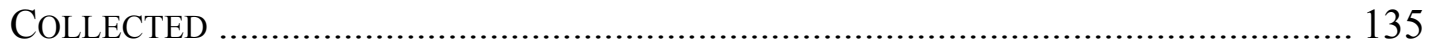

Figure 7.13 Times Series Plot of the Scores for PLS-Model 2 Component 2 ... 136

FIGURE 7.14 DMODX......................................................................................... 138

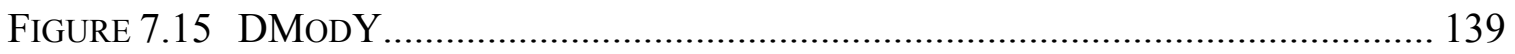




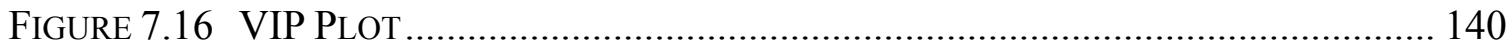

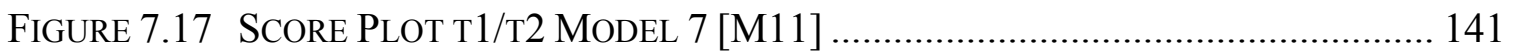

FIGURE 7.18 DMODX PLOT FOR PLS-MODEL 7 [M11] …........................................ 142

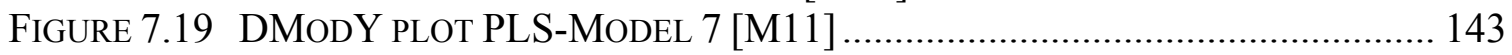

FigURE 7.20 LOADING PLOT FOR MODEL 7 [M13]................................................... 144

Figure 7.21 Score Plot With Treatment \#1 Pattern 1 to 12 IN THE Fourth

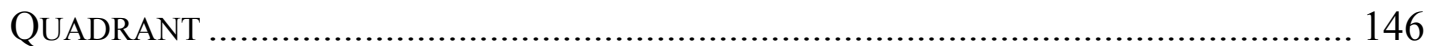

Figure 7.22 Combustion Temperature Time Series Plot....................................... 147

Figure 7.23 T[1] AND T[2] Time SERIES Plot ......................................................... 147

Figure 7.24 Contribution Plot For ObSERVATION 11 .......................................... 148

Figure 7.25 Outlet Temperature Time Series Plot - Circle Shows DroP in

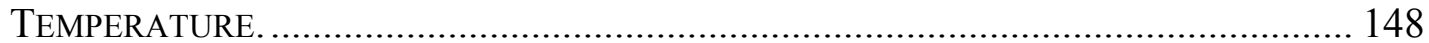

FiguRE 7.26 CONTRIBUTION Plot FOR OBSERVATION 135 ........................................ 149

FigURE 7.27 VARIABLE INFLUENCE ON PROJECTION (VIP)......................................... 150

FIGURE 7.28 COEFFICIENTS (CS) FOR PLS MODEL 7 [M13] ........................................ 151

FigURE 7.29 VALIDATE MODEL WITH 20 PERMUTATIONS ............................................. 153

Figure 7.30 Outlet Moisture Content Predicted Scatter Plot ……………...... 155

Figure 7.31 Score Plot Predicted Scores for Dryers and HeAt SOURCE............... 155

Figure 7.32 DMODX FOR PREDICTION SET 1 ………............................................ 156

FigURE 7.33 DModY For PREDICTION SET 1 ……….............................................. 156

Figure 7.34 Outlet Moisture Content Predicted Scatter Plot - Prediction Set

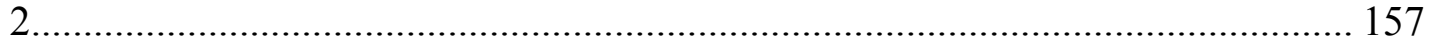

Figure 8.1 SHEWHART CHARTS FOR EACH INFLUENTIAL VARIABLE TO MONITOR DRYER

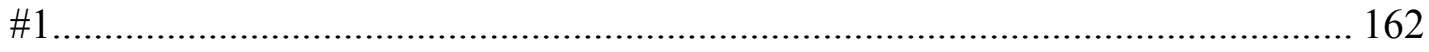

Figure 8.2 Control Charts of Correlated VARIABles. SCATter Plot SHOWS OUTLIER IN LOWER RIGHT CORNER ............................................................... 163

Figure 8.3 SheWHART CHART FOR DRYER SCORES - DATA SET JAN 14 \& 15, 2004 ..... 164

Figure 8.4 Shewhart Chart For Hotelling T ${ }^{2}$ - Data SET JAN $14 \&$ 15, 2004 ...... 164

Figure 8.5 OutLet Moisture Contents Jan 16, 2004 00:00 HRS to 16:00 HRS......... 165

Figure 8.6 SheWhart Chart For SCORE T[1] - Data SET JAN 16, 2004 .................... 166

Figure 8.7 Hotelling T² CONTROL CHART FOR JAN 21, 2004 12:05 AM to 1:05 AM 167

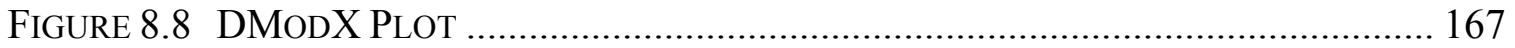

Figure 8.9 Plot of Actual vs. Predicted MC for Feb 6, 2004 data ...................... 168

FIGURE B. 1 TIME SERIES PLOT OF MOISTURE CONTENTS FROM A ROTARY, TRIPLE PASS

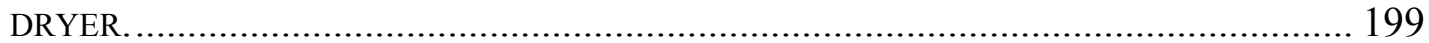

FIGURE B.2 AUTOCORRELATION FOR MOISTURE CONTENT TIME SERIES DATA WITH A 95\%

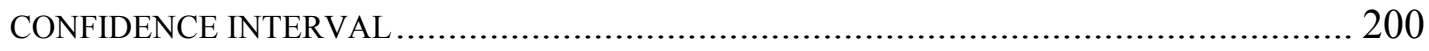

FIGURE B.3 SHEWHART INDIVIDUALS CONTROL CHART FOR MOISTURE CONTENTS FROM A

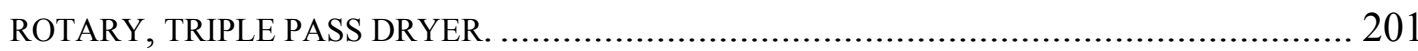

FigURE B.4 MOVING AVERAGE CONTROL CHART FOR MOISTURE CONTENTS FROM A ROTARY, TRIPLE PASS DRYER.................................................................... 202

FIGURE B.5 CUSUM CONTROL CHART FOR MOISTURE CONTENTS FROM A ROTARY, TRIPLE

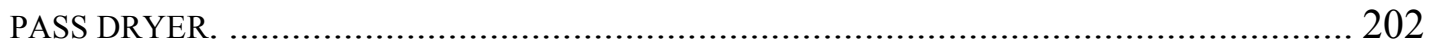

FIGURE B.6 EWMA CONTROL CHART FOR MOISTURE CONTENTS FROM A ROTARY, TRIPLE PASS DRYER. 203 
FIGURE B.7 ARIMA $(1,0,2)$ MODEL WITH ACTUAL MOISTURE CONTENTS FROM A ROTARY,

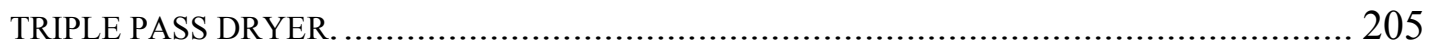

FIGURE B.8 SHEWHART INDIVIDUALS CONTROL CHART OF RESIDUALS FROM THE ARIMA

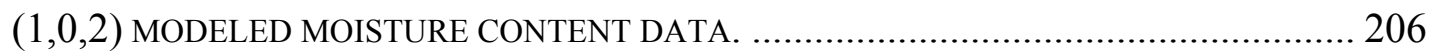

FIGURE B.9 ARIMA $(1,0,2)$ INDIVIDUALS CONTROL WITH LIMITS BASED ON THE ONE-

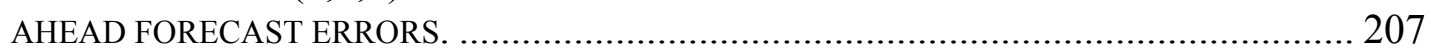

FIGURE B.10 ARIMA $(1,0,2)$ INDIVIDUALS CONTROL CHART FOR NEW MOISTURE CONTENT DATA FROM A ROTARY, TRIPLE PASS DRYER...................................................... 208 


\section{LIST OF SYMBOLS AND ABBREVIATIONS}

$\hat{T}$

$\wedge$

$\mathbf{y}$

$\hat{b}$

$\hat{\mathbf{E}}$

$\overline{\mathbf{x}}^{\prime}$

$\hat{\boldsymbol{\tau}}_{\alpha}$

$v$

$\hat{\mathbf{y}}_{j}$

$V(\hat{Y})$

$\sigma^{2}$

$r_{1}$

$\overline{m R}$

$\bar{X}$

$\nabla$

$\alpha$

$\mu$

$\lambda$

$\varepsilon_{\mathrm{t}}$

$\left(\mathbf{w}^{*} \mathbf{c}\right)$

$\Delta \mathrm{T}_{\mathrm{lm}}$

$\pi$

A

APA

AR

ARIMA

ARMA

b

B

C

$\mathrm{C}$

$\mathrm{c}_{\mathrm{a}}$

CI

Comp(A)

COST

$\mathrm{CS}$ set point of outlet temperature controller

least squares estimator for $\mathrm{y}$

coefficients, vector

residuals matrix $(\mathrm{M} \times \mathrm{K})$

mean

eigenvalue

correction factor that is a function of the number of observations and the number of components and is slightly larger than one

predicted estimate of $y_{j}$

variance of the predicted $\hat{Y}$

the $y$ error variance estimated from the sum of squares of the residuals divided by the degrees of freedom for PLS

autocorrelation at lag 1

average moving range

process average

backwards operator

drum slope

the process mean

damping factor

a sequence of independently and identically distributed random variables.

the X-weights $\left(\mathbf{w}^{*}\right)$ and, the Y-weights (c) plotted on the same scatter

plot

log-mean of the temperature difference between the hot air and the

product at the inlet and outlet respectively, C

pi

components

Engineered Wood Organization

autoregressive

autoregressive integrated moving average

autoregressive moving average

linear intercept

matrix of regression coefficients

Celsius

$\mathbf{Y}$ weights matrix $(\mathrm{A} \times \mathrm{M})$

weight vector of the y-data "a" components

continuous improvement

component A

considering one separate variable at a time

centered and scaled 


\begin{tabular}{|c|c|}
\hline $\begin{array}{l}\text { Cusum, } \\
\text { CV }\end{array}$ & $\begin{array}{l}\text { cumulative sum chart } \\
\text { cross validation }\end{array}$ \\
\hline $\mathrm{D}$ & drum diameter \\
\hline$d_{2}$ & value used to calculate control limits for X-bar charts \\
\hline D-Crit & critical distance to the model or maximum tolerable distance \\
\hline DF & degrees of freedom \\
\hline DModX & distance to the model $-\mathbf{X}$ data \\
\hline DModY & distance to the model - $\mathbf{Y}$ data \\
\hline DOE & design of experiment \\
\hline$e_{i k}$ & the X-residuals of observation i. \\
\hline EPC & engineering process control \\
\hline$e_{t}$ & prediction error \\
\hline EWMA & exponentially weighted moving average \\
\hline $\mathrm{F}$ & solids feed rate or fixed feed rate \\
\hline $\mathrm{F}$ & Y residuals \\
\hline $\mathrm{F}$ & Fahrenheit \\
\hline$f_{a}$ & residual vector of $y$ for " $\mathrm{a}^{\text {th" }}$ component \\
\hline GTS & heat source \\
\hline $\mathrm{H}$ & drum holdup \\
\hline $\mathrm{H}$ & residuals \\
\hline HMI & human machine interface \\
\hline Hotelling $\mathrm{T}^{2}$ & squared function of the PC-scores - $\mathrm{T}^{2}=(X-\bar{X})^{\prime} S^{-1}(X-\bar{X})$ \\
\hline I & integrated \\
\hline ID & induced draft \\
\hline IMA & integrated moving average \\
\hline IP2 $1^{\mathrm{TM}}$ & data historian by Aspen Tech \\
\hline $\mathrm{J}$ & joules \\
\hline $\mathrm{K}$ & constant \\
\hline $\mathrm{k}$ & number of $\mathrm{x}$-variables \\
\hline $\mathrm{K}$ & $\mathrm{X}$-variables \\
\hline $\mathrm{kg}$ & kilograms \\
\hline $\mathrm{L}$ & drum length \\
\hline $\mathrm{L}$ & length \\
\hline $\mathrm{m}$ & meter \\
\hline M & Y-variables \\
\hline M & number of y-variables \\
\hline $\mathrm{M}(3 / 8$ ths $)$ & one thousand square feet surface measure on a $3 / 8$ " basis \\
\hline MA & moving average \\
\hline MC-OD & moisture content oven dry basis \\
\hline MDF & medium density fiberboard \\
\hline MSE & mean square error \\
\hline MSPC & Multivariate Statistical Process Control \\
\hline N. & drum rotation speed \\
\hline $\begin{array}{l}\mathrm{n}_{\mathrm{f}} \\
\mathrm{NIR}\end{array}$ & $\begin{array}{l}\text { Number of lifting flights } \\
\text { near infrared }\end{array}$ \\
\hline
\end{tabular}




\begin{tabular}{|c|c|}
\hline OD & oven dry \\
\hline OSB & Oriented Strand Board \\
\hline OVAT & one variable at a time \\
\hline $\mathrm{P}$ & loading matrix (A x K) \\
\hline $\mathrm{p}_{\mathrm{a}}$ & loadings, $\mathrm{a}=$ components \\
\hline PCA & Principle Component Analysis \\
\hline $\mathrm{PC}_{\mathrm{A}}$ & principle component or dimension, A \\
\hline PC & principle component \\
\hline PCA-X & principal component analysis for $\mathrm{X}$ \\
\hline & pounds per cubic foot \\
\hline PID & proportional integral derivative \\
\hline PLC & programmable logic controller \\
\hline PLS & Projection to Latent Structures by means of partial least square \\
\hline pMDI & Polymeric diphenol dimethyl diisocyanate \\
\hline PRESS & $\begin{array}{l}\text { predictive residual sum of square, which is the summed squared } \\
\text { difference between predicted and observed values }\end{array}$ \\
\hline PS2-92 & performance standard for structural panels \\
\hline Q & total heat transferred \\
\hline$Q^{2}$ & goodness of prediction \\
\hline $\mathrm{Q}^{2}(\mathrm{Cum})$ & cumulative goodness of prediction \\
\hline $\mathrm{Q}^{2}$ & $\begin{array}{l}\text { the predictive ability of the model, Goodness of Prediction } \\
\mathrm{Q}^{2}=1 \text {-PRESS / SSX }\end{array}$ \\
\hline $\mathrm{q}_{\mathrm{s}}$ & rate of heat transfer to particles, $\mathrm{J} / \mathrm{s}$ \\
\hline $\mathrm{R}$ & slope of linear function between inlet/outlet temperatures. \\
\hline $\mathrm{R}$ & radius \\
\hline $\mathrm{R}^{2}$ & $\begin{array}{l}\text { Goodness of Fit; how much of the variation is explained by the model } \\
\mathrm{R}^{2}=1 \text { - RSS / SSX }\end{array}$ \\
\hline $\mathrm{R}^{2} \mathrm{VX}$ & explained variation for a variable, $\mathrm{x}$ \\
\hline $\mathrm{R}^{2} \mathrm{VY}$ & explained variation for a variable, $\mathrm{y}$ \\
\hline $\mathrm{R}^{2} \mathrm{X}$ & goodness of fit for $\mathrm{X}$ \\
\hline $\mathrm{R}^{2} \mathrm{X}(\mathrm{Cum})$ & cumulative goodness of fit for $\mathrm{X}$ \\
\hline$R^{2} Y$ & goodness of fit, $y$-variables \\
\hline $\mathrm{R}^{2} \mathrm{Y}$ & goodness of fit of $Y$ \\
\hline $\mathrm{R}^{2} \mathrm{Y}(\mathrm{Cum})$ & cumulative goodness of fit for $\mathrm{Y}$ \\
\hline RMSEP & root mean square error or prediction \\
\hline RSS & residual sum of squares \\
\hline RTD & resistance temperature device \\
\hline s & second \\
\hline $\mathbf{S}$ & covariance matrix \\
\hline $\mathrm{S}^{2} \mathrm{OX}$ & residual observation variance \\
\hline SPC & statistical process control \\
\hline SSVX & residual variable variation. $S S V X=\sum_{i} e_{i k}^{2}$ \\
\hline
\end{tabular}




\begin{tabular}{|c|c|}
\hline SSVY & residual response variable variation $S S V Y=\sum_{i} f_{i m}^{2}$ \\
\hline $\mathrm{SSX}_{\text {tot.corr }}$ & total variation in the $\mathbf{X}$ matrix after mean centering \\
\hline $\mathrm{t}$ & mean residence time of the particles \\
\hline $\mathbf{T}$ & score matrix $(\mathrm{M} \times \mathrm{A})$ \\
\hline $\mathbf{T} * \mathbf{P}$ & matrix product which models the data structure \\
\hline $\mathrm{t}_{\mathrm{a}}$ & scores, $\mathrm{a}=$ components \\
\hline $\mathrm{t}[1]$ & scores, component 1 \\
\hline $\mathrm{Ti}$ & inlet temperature, ${ }^{\circ} \mathrm{F}$ \\
\hline$T_{i}$ & inlet temperature \\
\hline $\mathrm{T}_{\mathrm{o}}$ & outlet temperature, ${ }^{\circ} \mathrm{F}$ \\
\hline $\mathrm{T}_{\mathrm{w}}$ & wet bulb temperature, ${ }^{\circ} \mathrm{F}$ \\
\hline $\mathrm{U}$ & volumetric heat transfer coefficients, $\mathrm{W} / \mathrm{m}^{30} \mathrm{C}$ \\
\hline $\mathbf{U}$ & $\begin{array}{l}\text { resulting matrix when scaling the principle component scores for each } \\
\text { component to length one }\end{array}$ \\
\hline $\mathbf{U}$ & Y scores matrix $(\mathrm{N} x \mathrm{~A})$ \\
\hline $\mathrm{u}_{\mathrm{a}}$ & Y score vector for "a" components \\
\hline V & gas velocity through the drum. \\
\hline $\mathrm{V}_{\mathrm{d}}$ & Volume of dryer drum, $\mathrm{m}^{3}$ \\
\hline VDP & vertical density profile \\
\hline VIP & variable influence on projection \\
\hline VOC & volatile organic compounds \\
\hline $\mathrm{w}^{*}$ & $\mathbf{X}$ variable weights \\
\hline $\mathbf{W}_{\mathbf{a}}$ & $\mathrm{X}$ variable weight for the $\mathrm{a}^{\text {th }}$ component \\
\hline W.C. & water column \\
\hline $\mathrm{X}$ & outlet moisture content, $\% \mathrm{MC}$ \\
\hline $\mathbf{X}$ & matrix $\mathrm{n}$ x $\mathrm{k}$ for $\mathrm{x}$-variables \\
\hline $\mathbf{X}^{\prime} \mathbf{X}$ & correlation matrix \\
\hline $\mathbf{X}_{\mathrm{a}-1}$ & preliminary residuals matrix \\
\hline $\mathrm{X}_{\mathrm{ik}}$ & observation i, variable $\mathrm{k}$ \\
\hline$X_{\text {in }}$ & Moisture content \\
\hline $\mathbf{Y}$ & matrix $\mathrm{n} \times \mathrm{m}$ for $\mathrm{y}$-variables \\
\hline $\mathbf{Y}$ & Matrix of $\mathrm{Y}(\mathrm{N} x \mathrm{M})$ \\
\hline$y_{\text {im }}$ & observation i, variable m \\
\hline $\mathrm{Z}_{\mathrm{it}}$ & observation i, variable t \\
\hline
\end{tabular}




\section{INTRODUCTION}

"The significant problems we face cannot be solved at the same level of thinking we were at when we created them." Albert Einstein

Oriented strand board (OSB) is a structural, wood composite panel product that has seen significant growth in the United States residential housing market over the past ten years. In 2000 OSB accounted for over $50 \%$ of all structural panels produced, and by the end of 2003 OSB had increased to $57.9 \%$ (Panel World 2004).

The reasons for the rapid growth and adoption of OSB can be attributed to lower costs, better quality, and aggressive marketing.

OSB production costs are lower than plywood as the processes are more automated requiring less labor. The labor requirement is only about twenty percent of that of plywood. In addition, log recovery is greater for OSB due to the utilization of the entire log and not having a residual plywood peeler core. These lower costs result in OSB being more profitable even with lower market pricing than plywood.

The switch from plywood to OSB is occurring due in part to the decline over the past decade in the quality of softwood plywood as a structural panel. This decline is due to the reduction in the availability of quality veneer as the quantity of veneer logs has been curtailed with the changes to the U.S. Forest Service policy on logging in the National Forests. To compound the issue the demand for quality structural veneers by the newer, laminated veneer lumber segment has further stressed the supply of quality veneer.

Improved OSB panel quality with less delamination and less warp typically found in plywood is another reason for the switch. In addition edge swell, a common complaint heard in the past with OSB, has been reduced with the newer "OSB-type" products such 
as J.M. Huber's Advantech, L.P.'s Top Notch, and Weyerhaeuser's Structurwood EDGE Gold.

Finally, marketing has entered the picture in speeding the adoption of OSB with new proprietary design values for "OSB-type" products such as TJ-Performance Plus Panel ${ }^{\circledR}$. This product sanctions a floor design system's approach with engineering design values approved by the International Code Council for both wood I-joists and the floor panel. Marketing has also "sweetened the pot" by offering a limited lifetime warranty on OSB products.

As products mature and become "commodity-like" where consumer distinction is blurred, price erosion typically follows. Businesses must then improve process efficiencies to reduce costs in order to maintain an attractive profit margin and return on investment for their stockholders. This means each step of the production process must be reviewed, analyzed, and improved to allow the business to remain competitive, much like many companies are doing with Six Sigma, a quality methodology used to improve products and processes.

The OSB production process consists of seven basic processing steps - log preparation, strand production, strand drying, strand blending, mat forming, mat pressing, and finishing. Each of these steps affects the quality of the final product as well as the cost and must be understood. There is a need to increase the understanding of each of these steps and their trade-offs as well as gain an appreciation for the relationships between the processing steps. For example what is the trade-off or consequence of adding more "particle-type" furnish to the OSB board if additives must be increased to ensure 
board quality? What about the trade-off or cost benefit in using denser hardwoods that could be used as long as panel density is increased?

One of the seven steps, strand drying, is least understood and managed by OSB operations. OSB production technology has evolved from other sectors of the wood products industry and from other industries. Strand drying on the other hand has not really evolved. The same technological methods used for drying agricultural grains in the US in the late 1950 's are still used for drying OSB strands.

Strand moisture content is a key process variable in the production of quality OSB. In the strand blending process, strand moisture content affects phenol formaldehyde resin curing with "wash-out" and "wash-in" of the resin occurring with "too high" and "too low" moisture contents respectively. In the mat forming process, varying strand moisture contents lead to varying mat weights that lead to varying finished panel thickness. In the mat pressing process, low strand moisture contents reduces mat consolidation and compaction and this lack of mat consolidation affects strand to strand contact and bonding which adversely affects strength properties. High moisture content strands, on the other hand, lead to "blows" or delamination.

Drying process improvement with better strand drying technology could reduce OSB production costs even further by reducing resin costs, mat densities, and press cycles while providing safer operations with fewer dryer fires. Reduced resin costs could be possible by controlling the application rate to actual bone dry weight of strands. Reduction in mat density targets could occur, as more uniform mat moisture results in more uniform mat weights with fewer low weight panels that have low strength properties. Reduced press cycles could be possible with more uniform mat moisture, as 
there would be less high weight mats that have a tendency to "blow"; thus increasing press productivity resulting in reduced costs. Finally better drying technology could mean safer drying operations with fewer dryer fires.

The research discussed in this dissertation is concerned with improving the OSB manufacturing process by 1) modeling the overall process from stranding to finishing to understand each step and the trade-offs and cost, and 2) modeling the strand drying process to reduce moisture variation. "The goal of many process improvement studies is to find those process variables, $\mathrm{X}^{\prime}$ s both controlled and uncontrolled, that affect the process outputs, Y's" (Snee 2002). The objective of this research is to do likewise, to gain an understanding of the process variables that affect the process outputs, but to go an extra step in developing first an overall OSB plant model to understand the steps and the trade-offs followed by a drying model so the output moisture content is more consistent.

Snee (2002) commented that "useful models should practice the principle of parsimony" or simplicity. He discussed "four types of models, which differ in their level of complexity and sophistication. 1) The key variables and direction of their effects, positive and negative, are known. 2) The key variables and direction and magnitude (linear effect) are known. 3) The prediction equation / model and the key variables, magnitude and direction of effects and functional form such as interaction and squared terms are known. 4) Theoretical models based on scientific and engineering fundamentals." The research in this dissertation targeted the first three models discussed by Snee. The first was the OSB Mill Process Flow Model that used a flow chart process, the second was the OSB Mill Model that used a Microsoft Excel ${ }^{\circledR}$ program, and the third was the OSB Dryer Model that used a multivariate modeling technique, projection to 
latent structures by means of partial least squares (PLS). In addition a statistical process control technique was used to monitor the drying process for shifts and trends and to alert management when it may be time to modify the drying model. 


\section{OBJECTIVES AND DISSERTATION STRUCTURE \\ "All Models are wrong, but some models are more useful than others." Anonymous}

\subsection{Objectives}

The objectives of the research described in this dissertation focused on various aspects of the OSB manufacturing process through a series of models - beginning with a very general model and each subsequent model progressively becoming more specific. The research began with study of the overall OSB manufacturing process and the development of a general process flow diagram model aimed at increasing the understanding of the various processing steps in an effort to continuously improve the process. The research was then narrowed with an investigation into modeling the OSB manufacturing process through the development of an OSB mill model as a management tool to answer "what if" and trade-off type questions. Finally, the research focused on just the drying processing step by developing an OSB drying model along with a statistical process control technique to facilitate improving the consistency of dry strand moisture contents.

To accomplish the research, the following were developed: 1) an OSB mill process flow model of the process to increase understanding and gain an appreciation for the key process variables and their effects on quality and productivity; 2) an OSB mill model to assist manufacturing management with understanding "what if" and trade-off type opportunities; 3) an OSB drying model to identify the process variables that effect strand moisture content so the OSB strand drying process can produce more consistent dry strand moisture contents; and 4) a monitoring / quality control technique using a 
multivariate statistical process control (MSPC) to guide dryer parameter adjustments. The research included the following tasks:

1. Modeling the OSB process flow using a flow chart / diagram of the major processing steps.

2. Modeling the overall OSB production process using Microsoft Excel ${ }^{\circledR}$ program.

3. Modeling the drying process using the multivariate technique, Projection to Latent Structures by Means of Partial Least Squares (PLS) using SIMCA-P, the multivariate data analysis software program by Umetrics (2001).

4. Developing a monitoring / quality control method for the drying process using a multivariate statistical process control (MSPC) technique.

\subsection{Structure of the Dissertation}

Chapter 3 contains a summary of rotary dryer drying research and rotary dryer operation to include dryer design models. A discussion on multivariate data analysis is also contained in this section. It includes the projection technique, the principal component analysis (PCA), and Projection to Latent Structures by means of Partial Least Squares (PLS).

Chapter 4 introduces the methods and materials used in the development of the various models along with the MVDA method used in developing the OSB dryer model.

Chapter 5 presents the OSB mill process flow model with the key process control variables and their effects both positive and negative.

Chapter 6 introduces the OSB mill model as a management tool. 
Chapter 7 presents the dryer model, outlining the variables of influence, the mathematical model, and the validation of the model.

Chapter 8 describes the MSPC technique to monitor the dryer process model developed in Chapter 7.

Chapter 9 is the conclusion of the dissertation; while chapter 10 provides recommendations for future research.

Appendix A is the OSB Mill Model Excel ${ }^{\circledR}$ program.

Appendix B is a discussion on univariate SPC techniques, and the effect of autocorrelated data on the occurrence of false "special causes"

Appendix C explains the ARIMA model used with autocorrelated data. 


\section{LITERATURE REVIEW}

"Learning is not compulsory...neither is survival.” W. Edwards Deming

Drying strands to consistent, final moisture contents is one of seven key process steps in manufacturing quality structural panels. Variation in the moisture content of the dried strands adversely impacts the "down stream" processing steps of blending, forming, pressing, and finishing.

Davis (1997) pointed out with liquid phenol formaldehyde (LPF) resin, high moisture contents can cause "wash out" while low moisture contents can cause "wash in" both causing bonding issues. Paulin (2003) noted with polymeric diphenol dimethyl diisocyanate (pMDI) resin, strand moistures below three percent will cause bonding problems while strand moistures over ten percent will increase internal mat pressure so press cycles, specifically the or venting phase, will have to be extended to prevent delamination.

High mat moisture variation in the forming process has been shown to increase the finished panel weight variation unless the forming process has a "sophisticated" control strategy with robust moisture meters to compensate for changing furnish moistures.

Maloney (1993) stated the variation of furnish moisture content in the pressing process leads to high internal, mat steam pressures during pressing and to "blows" or delamination in the final product with the product being downgraded.

Furnish moisture content variation in the finishing process was reported by Honda (1984) to cause finished product thickness variation with product grade, based on panel thickness, being adversely affected. 


\subsection{Rotary Drying}

\subsubsection{Rotary Dryer Heat and Mass Transfer}

Rotary dryer heat transfer has been studied empirically by a number of researchers. Van Krevelen and Hoftijzer (1949) commented that the total heat transferred, Q, increases with drum rotation speed, N. Friedman and Marshall (1949) found with a rotary dryer that increasing the air velocity increased the total heat transferred. Seaman and Mitchell (1954) reported air temperatures in the lower half of the drum were consistently higher than in the upper half and deduced that the air was entrained downward by the cascading particles.

Kamke (1983) commented that heat transfer calculations in rotary dryers have been studied from either 1) an analysis based on an empirically derived overall heat transfer coefficient or 2) an approximation analysis using individual particle heat transfer coefficients. The first is characterized by:

$$
q_{s}=U V_{d} \Delta T_{l m}
$$

where: $q_{s}=$ rate of heat transfer to particles, $\mathrm{J} / \mathrm{s}$

$$
\begin{aligned}
& \mathrm{U}=\text { volumetric heat transfer coefficients, } \mathrm{W} / \mathrm{m}^{3 \circ} \mathrm{C} \\
& \mathrm{V}_{\mathrm{d}}=\text { volume of drum, } \mathrm{m}^{3} \\
& \Delta T_{l m}=\text { log-mean of the temperature difference between the hot air and the } \\
& \text { product being dried at the inlet and outlet respectively, }{ }^{\circ} \mathrm{C}
\end{aligned}
$$

The second requires assumptions concerning the geometry of the system, particularly concerning the dynamics of the gas-particle interaction. 
Kamke (1983) discussed the work of McCormick (1962) in which it was reported the heat transfer coefficient varies with the characteristics of the solids, flight arrangement, flight capacity, drum speed, and drum holdup. Sharples et al. (1964) rotary dryer model, discussed by Kamke (1983), had a value of U (volumetric heat transfer coefficient) that was proportional to the cascade rate, drum speed, and the gas flow rate. The work of Miller et al. (1942) was also discussed by Kamke (1983) in estimating the rate of heat transfer in a rotary dryer. The rate of heat transfer was calculated as the sum of the sensible heat acquired by the solids and liquid water plus the latent heat of evaporation of the water driven off. An overall heat transfer coefficient was determined using equation (3-1) above by assuming a mean driving force to be the log-mean temperature difference between the air and solids evaluated at the inlet and outlet of the drum.

Research in mass transfer in rotary dryers is even more sparse than heat transfer and is typically studied in the context of overall drying and as such is really a study of simultaneous heat and mass transfer. Thorne's (1979) work discussed by Kamke (1983) developed a vapor diffusion model to describe the drying mechanism. Drying was assumed to occur during the particle's fall period and during the time of travel of the particle on the lifting flights. While resting on the flights, the particles are believed to be in a "soaking" period in which moisture was not allowed to cross the particle surface. Thorne concluded that gas velocity, as it affects particle mechanics, is an intricate component of the rotary drying process.

Commercial rotary dryers it is further complicated by the "cascade" motion of the solids though the dryer. The cascade cycle can be divided into cascading and resting in 
the flights. In the cascading phase the solids are subjected to heat and mass transfer process. In the resting period the processes are slowed and possibly stopped entirely. Kamke (1983) referred to the resting period as "soaking". During this period the mean particle temperature remains constant. In his work he found the total amount of heat transferred to the particle bed was negligible.

Due to the cascading process it is difficult to describe the drying process as a whole by the mathematical equations of heat and mass transfer. This is because it involves not only the properties of the solids or wood and the drying gases, but also the design and operating characteristics of a rotary dryer. These characteristics may be the number of dryer flights, the flight cascade pattern, the ratio of falling to resting times, the physical size of the dryer, and many others (Mujumdar 1989).

With the difficulty in using heat and mass transfer equations, the research discussed in this dissertation was designed to empirically determine the relationships of the drying variables for rotary dryers and the outlet temperature and moisture content.

\subsubsection{Rotary Dryer Operation Theory}

Convection drying is the most common mode used in drying particulate solids. It is also referred to as direct drying. These types of dryers are air suspension dryers such as rotary, fluid bed, flash, or spray dryers. In convection drying, heated air or gas flowing over the surface of the solid supplies the heat energy. Heat for evaporation is supplied by convection to the exposed surface of the material and the evaporated moisture is carried away by the drying medium. In the initial constant rate drying period, that is drying where the surface moisture is removed, the solid surface takes on the wet bulb temperature corresponding to the air temperature and the humidity conditions at the same 
spot. In the falling rate period, the solids temperature approaches the dry bulb temperature of the air (Mujumdar 1989).

The basic features used in design of a rotary dryer are: solids feed rate $\mathrm{F}$, moisture content $\mathrm{X}_{\text {in }}$, drum diameter $\mathrm{D}$, drum length $\mathrm{L}$, drum slope $\alpha$, rotational speed $\mathrm{N}$, lifting flights number $\mathrm{n}_{\mathrm{f}}$, flight profile, drying gas direction, co-current or countercurrent, and velocity $\mathrm{V}$ through drum. Based on these features, the operational parameters of drum holdup or drum loading $\mathrm{H}$ and outlet moisture content $\mathrm{X}_{\text {out }}$ can be theoretically derived (Mujumdar 1989).

Three basic processes occur within a rotary dryer and are defined as follows: 1) movements of the particles as they progress by cascade and kiln or sliding motion through the drum length, 2) heat transfer from the hot gases to the particles providing the latent heat of vaporization to the moisture within the particles, and 3) mass transfer of the moisture from within the particles to the surface and then to the hot gases in the drum.

Although the principal objectives of the drying process are the simultaneous heat and mass processes as defined in \#2 and \#3 above, to adequately analyze their respective rates requires an understanding of the particle dynamics through the drum. Therefore most of the investigations into rotary drying operations have focused on understanding the movement of particles down the drum length. In fact until a satisfactory model of the particle dynamics is provided, the heat and mass transfer phenomena theories are limited in their applications. Furthermore, the particle movement forms the basis for the development of the residence time model that gives the average time the particles spend in the drum, as well as the distribution about the average (Mujumdar 1989). 
Particles progress through the drum in a series of cascades and a sliding, rolling, or kiln motion between flights down the walls of the drum. In each cascade, the average particle is captured by the flight in the lower half of the drum and carried stationary in the flight into the upper half of the drum. When its angle of repose in the flight is greater than its equilibrium angle, the particle cascades off the lip of the flight and falls through the hot gases back into the lower half of the drum. In falling through the gases, the particles are subjected to drag from the gases and are carried forward along the drum length. Mujumdar (1989) breaks the particle movements over the length of the drum into three components:

1) forward movement of the cascading particles due to the drum angle,

2) forward movement due to the drag on the particles from the drying gases, and

3) kiln or sliding rolling action between flights in the lower half of the drum.

The angle of repose is the maximum angle particles will sustain with a horizontal surface when they are poured from a container. This angle depends on factors such as particle size, particle geometry, moisture content that will affect their "stickiness" and the surface coefficient of friction. Kamke (1983) reported that particles with 146 percent moisture content have a mean angle of repose eight degrees higher than for particles with 10 percent moisture content. Particle geometry affects the angle of repose with granular particles approaching a more free flowing state with a lower angle. Long and curly particles or strands tend to interlock and strengthen the bridging of the material thus sustaining a greater angle of repose.

There are three forces acting on the rolling particles. These are gravitational, frictional, and centrifugal. It has also been reported that in observing wood particles 
cascading in a rotary drum, the flow is not even but rather an intermittent release of particles.

\subsubsection{Drum Particle Holdup}

In analyzing the operation of the rotary dryer, the quantity of particles in the drum during steady-state operation is referred to as drum holdup, $\mathrm{H}$ and is a key parameter in dryer analysis. For a fixed feed rate F, the mean residence time of the particles, $t$, is related as:

$$
t(\mathrm{sec})=\frac{H}{F}
$$

where: $\mathrm{H}$ is in $\mathrm{m}^{3}$ or $\mathrm{kg}$, and

$\mathrm{F}$ is in $\mathrm{m}^{3} / \mathrm{sec}$ or $\mathrm{kg} / \mathrm{sec}$

Drum holdup has been described as a function of the number of flights and the flight holdup. Industry experience calls for the drum holdup to be in the range of 10-15\% of the total drum volume Mujumdar (1989).

A drum may be underloaded, design loaded or overloaded. Underloading is a highly inefficient operation as the gas flow will take the path of least resistance and will flow down the drum where the particles are not cascading. With an overloaded drum, there is a rolling load of particles in the bottom half of the drum that bypasses the flights. This will result in under dried particles and an unacceptable spread of moisture contents. A fully loaded drum is one in which the rotating flights are full when they pass upward through the drums horizontal axis, thus ensuring cascade right across the drum and minimizing the rolling load Mujumdar (1989). It is generally preferable to operate a drum dryer in slightly overloaded conditions. This is because the cascade rate falls off rapidly 
at underloaded conditions, thus allowing the gases to bypass the cascading particles. Mujumdar (1989) reported when a drum is at $96 \%$ of the design load, the flights are only $75 \%$ loaded as they pass upward through the horizontal drum axis. Kamke (1983), on the

other hand, reported that rotary dryers used for drying wood articles are operated at below design holdup of the flights rather than overloaded. Overloading causes a decrease in the gas-particle interaction, requiring additional residence time to achieve the desired degree of drying. Underloading will actually increase the angle at which cascading begins, the furnish actually stays on the flight longer before falling, thus giving more fall time in which the particles are exposed to the gas stream.

\subsubsection{Residence Time}

Mujumdar (1989) related residence time empirically to five principal parameters, drum diameter $\mathrm{D}$, length $\mathrm{L}$, slope $\alpha$, drum speed $\mathrm{N}$, and the gas velocity $\mathrm{V}$ through the drum. It is also known that other factors such as drum holdup or loading $\mathrm{H}$, particle geometry, flight profile, and particle distribution on the flights contribute to residence time.

One widely accepted equation for residence time, $t$, is

$$
t=\frac{L}{f(H) D N(\alpha-k V)}
$$

Where: $f(\mathrm{H})$ is 3.14 for a heavily loaded drum and 2 for a lightly loaded drum, and $\mathrm{K}$ is a constant equal to 0.001 to $0.008 \mathrm{sec} /$ foot.

The assumption is the horizontal drift of the cascading particle is linearly related to the air velocity and the length of fall. The distance the particle moves forward in a 
drum is dependent on the height of the fall, the slope of the drum, and the gas velocity Mujumdar (1989).

Kamke (1983) measured residence time using a radioactive tracer technique. He reported an increase in drum speed decreased the average residence time but it was not proportional. The effect was most pronounced for the 1.2 meter diameter drum at drum speeds less than four revolutions per minute. It was also reported that as the drum diameter increased the residence time decreased at a constant drum speed. This is a result of the longer distance a particle falls with more time for the gas-particle interaction which resulted in more longitudinal movement per cascade. The residence time was also decreased as the gas velocity increased.

Kamke (1983) found, for the particle sizes studied, during a cascade, the particles fall in curtains separated by relatively particle free areas. Within a curtain, particle contact and shielding with the bulkier particles can affect the flow. It was reported that the denser the curtain the more interaction; therefore, the individual particle flow characteristics are influenced by the bulk particle flow. Larger particles did not have a longer residence time but there was an interaction between particle size and residence time.

Kamke (1983) also found that as the fractional drum holdup increased (the drum is more fully loaded) the residence time decreased. To increase the fractional drum holdup, the feed rate must be increased. The increase in the feed rate is proportionally greater than the increase in the drum holdup, resulting in a decrease in the residence time. 


\subsubsection{Rotary Dryer Design Models}

The industry has been moving from designing dryers based on experience developed over many years as well as pilot plant studies to the use of computer models to design dryers. There are several weaknesses to the computer models, however, as discussed by Mujumdar (1989). These are:

1) Kiln or rolling load action is difficult to quantify and may account for over $50 \%$ of the movement of the particles through the dryers.

2) Effect of loading, more specifically underloading, is difficult to incorporate into a model, so most models are set up for either design or overloading.

3) Effect of gas velocity is probably the single greatest weakness. The physical situation within the dryer is quite complex, with cascading curtains moving across the drum at right angles to the gas flow.

Industry uses models to help with "debottlenecking" the process where the dryer might be the limitation in getting more production. The models do provide some value in the calculation of residence time. In this case the easiest variable to adjust would be drum speed and then investigate its effect on residence time.

From a heat and mass transfer viewpoint, Mujumdar (1989) believed the parameter to be maximized would be the proportion of total drum holdup cascading through the drying gases, as the particles in the bottom of the drum or in flights are relatively inactive. This would suggest a short fat dryer with the longest distance of fall is optimum; however, fuel costs would be higher. Consequently, longer dryers with lengthdiameter ratio of 6 to 8 are usually found for commercial dryers. 


\subsubsection{Dryer Variables Effect on Outlet Moisture Content}

Kamke (1983) reported that the inlet particle moisture content has the greatest affect on predicted outlet moisture content. Then in decreasing order of influence were: blend box gas temperature, drum diameter, air leakage, drum length, gas volumetric flow rate, particle size, particle sphericity, drum speed, and angle of repose.

Increasing the gas volumetric flow rate causes a particle to pass through the drum quicker, i.e. fewer cascades. However, an increase in the flow enhances the convection rate of heat transfer. "These are counteracting affects on the extent of drying, and thus an optimal gas flow must exist" (Kamke 1983).

Shinskey and Fadum (1980) presented a relationship between the outlet moisture content of the wood and the inlet, outlet, and wet bulb temperatures. A differential slice of a dryer is taken and integrated over the length to get the following equation:

$$
X=K \ln \left(\frac{T_{i}-T_{w}}{T_{o}-T_{w}}\right)
$$

where: $\mathrm{X}=$ outlet moisture content, $\% \mathrm{MC}$

$$
\begin{aligned}
& \mathrm{T}_{\mathrm{i}}=\text { inlet temperature, }{ }^{\circ} \mathrm{F} \\
& \mathrm{T}_{\mathrm{o}}=\text { outlet temperature, }{ }^{\circ} \mathrm{F} \\
& \mathrm{T}_{\mathrm{w}}=\text { wet bulb temperature, }{ }^{\circ} \mathrm{F} \\
& \mathrm{K}=\text { constant. }
\end{aligned}
$$

He believed outlet moisture content could be maintained constant if the ratio of inlet, outlet, and wet bulb temperature could be maintained constant. If the temperatures could be accurately measured and adjusted accordingly to maintain the ratio, the outlet moisture would be more consistent. In many cases, accurately measuring the wet bulb 
temperature is difficult with the contaminated air stream at the exit of the dryer. The wet bulb temperature is a function of relative humidity and inlet temperature and could be calculated if a reliable relative humidity sensor could be obtained. Dewcon, Inc. does manufacture such a device, but as pointed out by Mujumdar (1989), these devices are high maintenance items.

Shinskey and Fadum (1980) showed that increasing dryer load can be compensated for by increasing the inlet temperature and outlet temperatures to maintain set point outlet moisture. The relationship can be approximated by a linear function at elevated temperatures. The ratios are different for different outlet moistures. The assumption is that the outlet temperature is a function of inlet temperature and the wet bulb temperature is approximately linear at higher temperatures. Shinskey and Fadum (1980) used the relationship to calculate the set point for the outlet temperature controller.

$$
\hat{T}_{o}=b+R T_{i}
$$

where: $\hat{T}_{o}=$ set point of outlet temperature controller

$$
\begin{aligned}
& \mathrm{b}=\text { linear intercept } \\
& \mathrm{R}=\text { slope of linear function between inlet/outlet temperatures. } \\
& T_{i}=\text { inlet temperature. }
\end{aligned}
$$

While the literature indicates multiple variables influence the operation of a rotary dryer and the outlet moisture content, many of these variables are applicable only when designing a new dryer system. The numbers of variables that influence final moisture content are greatly reduced, when looking at an existing drying system. The variables, based on Kamke (1983), Honda (1989), Maloney (1993) and Shinskey and Fadum (1980), with the most influence on the outlet moisture content are: inlet moisture content, 
gas volumetric flow rate, inlet temperature, strand geometry, strand feed rate, wet bulb temperature, and outlet temperature; strand feed rate and outlet temperature. Several of these variables were studied in this research.

\subsection{Multivariate Data Analysis ${ }^{1}$}

When asked what single event was most helpful in figuring out the theory of relativity, Albert Einstein was reported to have answered: "Figuring out how to think about the problem" (J. Trout 2000). The same dilemma, "how to think about the problem", is faced today by managers of industrial production facilities. These facilities have hundreds upon hundreds of industrial sensors and devices, automatically generating millions of data 24-hours a day, 7 days a week. Since "data are the not the same as information" these data need to be manipulated or processed to get information that can be used for continuously improving the process and products.

The purposes for measuring data on processes are designed to. 1) Provide information to gain a better understanding of the process and of relationships between the different parts of the process so they can be improved. 2) Yield information about the "state" of the process - recognizing trends, special causes, etc. - to keep the process under control. 3) Discover how the input variables and the process variables affect the product or output so improvements can be made. In short multivariate data analysis (MVDA) gets the data to "talk" and tell a story so the process can be modeled.

\footnotetext{
${ }^{1}$ The concepts contained in this section are patterned in thought and lay out based on the work by Eriksson et al.1999, Eriksson et al. 2001, Kachigan 1991 and Martens and Naes 1989. The basic concepts used to explain projection, PCA, and PLS are based on Eriksson et al. 2001. The basic mathematics behind PCA and PLS are based on Martens and Naes 1989 and Lindgren et al.1993. For more in depth review of the concepts discussed in this section all of these works are highly recommended. These works have numerous examples to highlight the concepts briefly discussed here.
} 
Until recently, measurement devices were relatively expensive and few were used; consequently the amount of data measured on processes was limited. The display, monitoring, and analysis of these few data were relatively simple, and a few runs charts of the data provided the information about the state of the process. Today, sensors and on-line instruments provide data from all parts of the process in many forms, often at very short time intervals, seconds. The masses of data are fed into computers and displayed in various charts and data stored for future analysis. The change from the situation with few, infrequent measurements, to the current situation with many, continuously measured variables has not altered the manner in which the process data are treated; consequently large losses of information occur. Creators of data historians have successfully helped production facilities store millions of data, but the use of the data to obtain information to improve the processes and products has often been lacking.

MVDA is used to analyze the masses of process data and is seen as the preferred analysis technique when dealing with the large volumes of data, particularly data that are correlated. MVDA provides easier to grasp graphical information about the state of the process, and the relationships between important process variables.

The MVDA methodology uses projection techniques referred to as Principal Components Analysis (PCA) and Projection to Latent Structures (PLS). They make efficient use of all pertinent data, with little loss of information. The methods are often applied to three basic problem types: 1) Overview of a data table, 2) classification or discrimination among groups of observations, and 3) regression modeling between two blocks of data (X and $\mathbf{Y}$ ). 
The traditional way to gain insight into the state of a process is to display important variables and their change over time. This works fairly well with up to say five to seven variables, but then becomes increasingly difficult to comprehend. This method is sometime referred to as considering one separate variable at a time, COST, or as one variable at a time, OVAT. It reveals little about the relationships between different variables. COST does not adequately examine multiple variables to see if they are in the same class or group since it only "eye-balls" one variable at a time.

In using COST the risk of a type I error, rejecting the null hypothesis when it is true or false positives, increases as the number of variables studied increases. If a significance level is assumed to be 0.05 , the risk can be expressed as:

$$
\text { Risk }=1-0.95^{K}
$$

where: $\mathrm{k}=$ number of variables.

If only one variable is examined the risk of type I error is $1-0.95^{1}=0.05$. But if the number of variables increases to five, the risk increases to $1-0.95^{5}=0.23$. Some industrial studies could be as large as 40 variables, and the risk increases to 0.87 . The more variables studied with the COST method the greater the chance of concluding there is a difference when there truly is not.

Another problem with studying just one variable $\mathrm{X}$ to predict $\mathrm{Y}$ is lack of selectivity in which no single $\mathrm{X}$-variable is sufficient to predict $\mathrm{Y}$. With multivariate methods, one can investigate the relations between all variables in a single context. These relationships can be displayed in plots as easy to understand as time series and pair-wise scatter plots. 
This section begins with an overview of MVDA and then a more specific discussion on two MVDA techniques - principal component analysis (PCA) and projection to latent structures by means of partial least squares (PLS). The overview first looks at multivariate data in general and then the concept of projection for k-variables with k-space and how to visualize this concept used in MVDA. The MVDA technique called PCA is reviewed including: 1) an overview of PCA data analysis, 2) a geometric interpretation of PCA, 3) the mathematics of PCA, and 4) an interpretation of a PCA model. The other MVDA technique - PLS - discussion is similar to the one for PCA but there is a more in depth discussion on the PLS model interpretation. If one is interested in more information about MVDA the following books are excellent resources - Eriksson et al. 2001, Eriksson et al. 1999, Kachigan 1991, Martens and Naes 1989, and Lindgren et al. 1993.

\subsubsection{Multivariate Data}

Multivariate data do have problems and challenges, but multivariate data analysis helps to minimize them. The first challenge is dimensionality, or simply stated, data overload as hundreds of variables are scanned and stored every one to sixty seconds. Multivariate data analysis can overcome this problem by using all the variables at the same time. Second is multicollinearity that arises because variables are approximate linear functions of other variables and therefore not independent of each other. Multiple linear regression may not be used because the data matrix must be full rank, i.e. the data must to be independent. Multivariate projection methods may be used to treat data with multicollinearity. The third challenge is dealing with noise or variation also referred to as experimental error. Individual measurements may be noisy and contain large variability 
that obscures important effects. Multivariate projection methods fortunately are "robust" to noise, and the data are "filtered" such that the systematic information or the effects are highlighted. By including many variables, the MVDA latent variables are stabilized, even if each of the measure variables is very noisy. The last problem is missing data. Many process data tables have gaps due to, for example, a faulty sensor or the data historian did not get the data from one of the scans. Projection methods tolerate moderate amounts of missing data both in the $\mathrm{X}$ and $\mathrm{Y}$ matrices. Around 10 to $20 \%$ missing data can be handled (Eriksson et al. 2001).

Multivariate data from processes can be categorized as controlled process variables, result variables, input variables, intermediate result variables, and uncontrolled variables.

The controlled process variables are related to the controlled settings of the process. These are variables that can be changed, thereby affecting the output of the process. The values of these variables are denoted by $\mathrm{x}_{\mathrm{ik}}$ (observation $\mathrm{i}$, variable $\mathrm{k}$ ).

- $\mathrm{x}_{\mathrm{i} 1}$ : the measured temperatures in dryers.

- $\mathrm{x}_{\mathrm{i} 2}$ : the fire box pressures in wood fired burners systems.

The result variables are the responses or outputs of the process. These variables measure important properties of the products coming out of the process and increase the ability to understand and optimize the process. The result variables are denoted $\mathrm{y}_{\mathrm{im}}$ (observation i, y-variable m).

- $\mathrm{y}_{\mathrm{i} 1}$ : dry strand moisture content $(\%)$

- $\mathrm{y}_{\mathrm{i} 3}$ : differential edge swell (\%). 
The inputs to the process or raw materials are denoted by $\mathrm{x}_{\mathrm{ik}}$ (observation $\mathrm{i}$, variable $\mathrm{k}$ ). These variables are often of great importance for the process and the product properties, but usually are difficult or impossible to control.

- $\mathrm{x}_{\mathrm{i} 3}$ : wood species

- $\mathrm{x}_{\mathrm{i} 4}: \log$ quality such as straightness

- $\mathrm{x}_{\mathrm{i} 5}$ : green strand moisture content

The intermediate result variables are denoted by $\mathrm{x}_{\mathrm{ik}}, \mathrm{y}_{\mathrm{im}}$, or $\mathrm{z}_{\mathrm{it}}$ (observation $\mathrm{i}, \mathrm{x}$ variable $\mathrm{k}, \mathrm{y}$-variable $\mathrm{m}$, or $\mathrm{z}$-variable $\mathrm{t}$ ). They are inputs or results that occur during a portion of the process but are not the final results. They may be treated as a result variable or an input variable.

- $\mathrm{y}_{\mathrm{i} 4}$ : outlet temperature for dryer 1

- $\mathrm{y}_{\mathrm{i} 5}$ : damper position for wood fired burner system

Uncontrolled variables are denoted by $\mathrm{x}_{\mathrm{ik}}$, or $\mathrm{z}_{\mathrm{it}}$. These variables can not be controlled but the information they provide may reduce the noise in the model.

- $\mathrm{x}_{\mathrm{i} 10}$ : ambient air humidity

- $\mathrm{x}_{\mathrm{i} 11}$ : temperature

- $\mathrm{x}_{\mathrm{i} 12}$ : month or season

\subsubsection{Principles of Projections}

The basics of MVDA may be outlined in terms of geometry of multidimensional spaces and the principles of projection. The data and the model can be represented as points, planes, and hyperplanes in these spaces. Two- and three- dimensional spaces can be easily comprehended, but higher dimensional spaces, k-spaces are impossible for the 
human mind to comprehend. The high-dimensional space is called K-space when working with $\mathrm{X}$-variables, and when working with $\mathrm{Y}$-variables, it is called M-space.

The simplest situation is when only two variables are studied $(K=2)$. This can be defined graphically as the two-dimensional, $x-y$ coordinate or Cartesian system. The coordinate system can be used to plot the data of two variables such as body height and body weight as shown in Figure 3.1. Each data point represents two variables, height and weight.

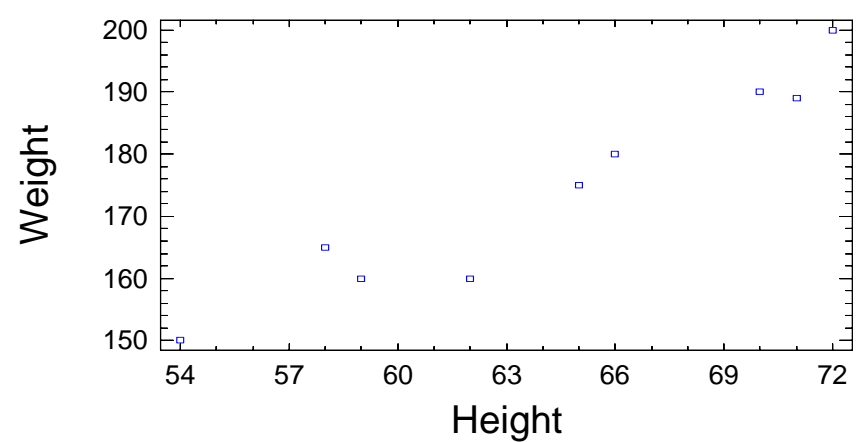

Figure 3.1 The x-y coordinate system depicting graphically the relationship between height and weight.

When three variables are studied $(K=3)$, the data can be viewed as $x-y-z$ coordinate system, as shown in Figure 3.2, where age has been added to the weight and height variables. Attempting to visualize this is a little more difficult than $\mathrm{k}=2$ so visualizing $\mathrm{K}$-space for $\mathrm{k}>3$ is unimaginable. 


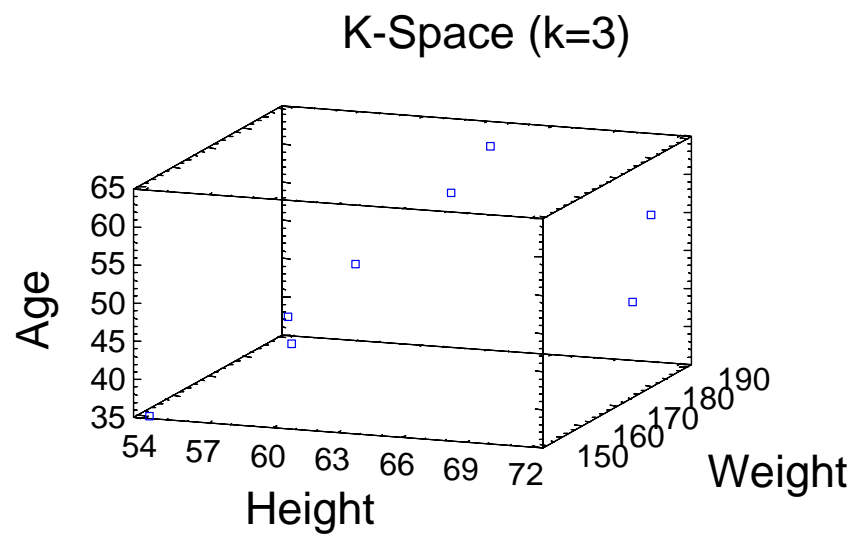

Figure 3.2 The $x-y-z$ coordinate system depicting graphically the relationship between height, weight, and age.

Each observation in the three variable data table is characterized as three numerical values. The three values of an observation are represented by one point in space, and the whole data table is represented as a point-swarm. The concept of three variables represented by a three-dimensional space can be extended to $\mathrm{K}$ variables in the $\mathrm{K}$-space. A K-space is constructed by letting each of the $\mathrm{K} \mathrm{X}$-variables define one orthogonal axis in a coordinate system. While K-space cannot be visualized, it is comparable to that of two- and three-dimensional space. The three-dimensional space may be used to model the K-space and illustrate multivariate analysis in terms of less complex spaces.

Analyzing multivariate data can be characterized as quantifying the description of the data swarm by the principle of projection. The principle of projection uses a model that could be simply viewed as a windowpane or plane in the three dimensional space. The windowpane is oriented in such a way that it provides a good overview of the data. A three-dimensional data table is converted into a two-dimensional space or plot by 
projecting the data swarm down to the windowpane. Geometrically, the projection of the data points is found by drawing a line from the point perpendicular to the space or windowpane as shown in Figure 3.3.

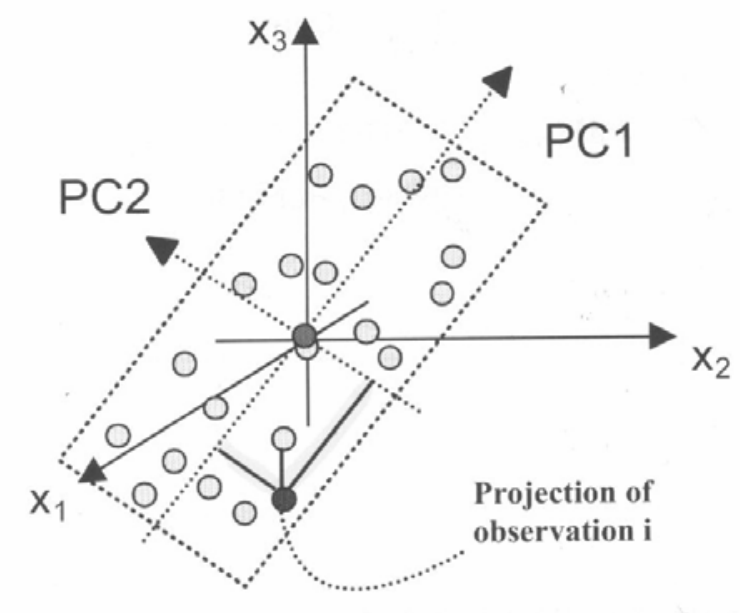

Figure 3.3 The plane is a window into the multidimensional space. Each observation is projected to the plane in order to get new coordinate values (scores) in the new coordinate system (Eriksson et al. 2001)

By looking at the location of each observation in the windowpane an overview of the data is possible, i.e., which observations are close to or far away from each other. The formation in the data can be seen as patterns, e.g. time trends, clusters, or deviating observations. The direction of the windowpane also gives information about which variables are important and which are not. It shows how the important variables combine to separate the clusters of observations or to define trends among the observations over time.

Assume a data table has 20 variables and 15 observations (Figure 3.4). For each of the observations, the values of the 20 variables have been measured. The K-space has 20 dimensions or axes because there are 20 variables. Each of the 15 observations become one point in the twenty-dimensional space, and together all 15 points form a 
swarm of points. A low-dimensional model, such as the windowpane, can approximate the shape of the data swarm by projecting each observation (data point) to the plane.

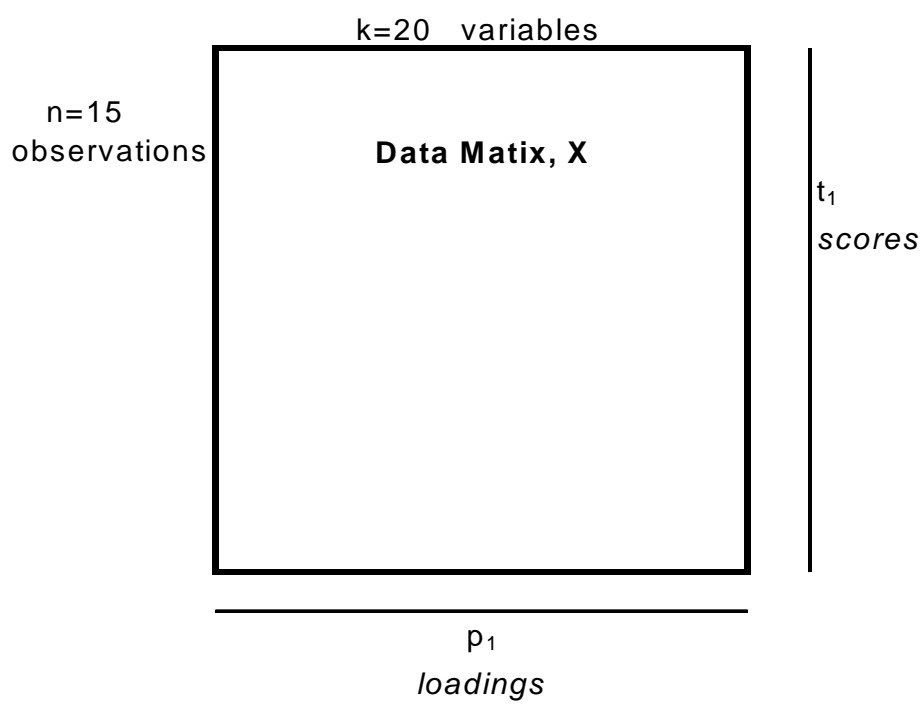

Figure 3.4 Data table or matrix (15 x 20) with scores for each observation and loadings for each variable.

Mathematically, a projection of a vector $\mathbf{y}$ into a subspace spanned by linearly independent vectors $\left(\mathbf{x}_{1}, \mathbf{x}_{\mathbf{k}}\right)=\mathbf{X}$ is defined by the linear operator $\mathbf{X}\left(\mathbf{X}^{\prime} \mathbf{X}\right)^{-1} \mathbf{X}^{\prime} \mathbf{y}$. It is said the vector $\mathbf{y}$ is projected into the space spanned by $\mathbf{X}$ (Martens and Naes 1989). This is the same operation used in linear regression to estimate the least squares value for $\mathbf{y}$. $\hat{\mathbf{y}}=\mathbf{X} \hat{\mathbf{b}}=\mathbf{X}\left(\mathbf{X}^{\prime} \mathbf{X}\right)^{-\mathbf{1}} \mathbf{X}^{\prime} \mathbf{y}$ is exactly the projection of $\mathbf{y}$ into $\mathbf{X}$-space. In other words, the estimator, $\hat{\mathbf{y}}$, is the vector in $\mathbf{X}$-space which is closest to the $\mathbf{y}$. It is also the computation method used to estimate $\hat{\mathbf{y}}$ that minimizes the sum of squares of residuals between the mathematical model and the data. 
The projection of data to the windowpane or two-dimensional plot is called a score plot, and it shows how the observations are projected down from twenty dimensions to two dimensions. The two-dimensional score plot is a summary of the relationships between the observations with the two dimensions referred to as score vectors, $t_{1}$ and $t_{2}$. A score, $t_{11}$, is a linear combination, row wise in the data matrix, or weighted average of all the values in the data row (Figure 3.4). The scores can also be thought of as the distances on the line in the plane.

The weights used to determine the scores are the loading values for combining the original variables to form the scores for the score plot. Geometrically the weights represent the direction in the K-space. The loading values are also plotted and referred to as the loading plot. "The loading plot is a summary of the relationships of the variables, and which variables are dominant. They also help to explain the patterns in the score plot. It is important to realize the two plots are complementary and superimposable, and the direction in one corresponds to the same direction in the other. The loading plot shows the orientation of the obtained windowpane in relation to the original variables. The plot can be thought of as reflecting the direction and extension of each original variable, as perceived when looking at them from the model windowpane. Variables that are most important for the model are found on the periphery of the loading plot and have large weight values. Conversely, non-influential variables are encountered around the origin of the plot $(0,0)$ " (Eriksson et al. 2001). Each variable gets a weight or loading value, $\mathrm{p}_{1}$ for each component or dimension as shown in Figure 3.4.

In summary, with the projection technique, "the measured variables are summarized by calculating new ones, called scores. These new variables are embedded in 
the measured ones, and therefore referred to as "latent variables". Using them to create a score plot gives a view of the configuration of the observations, "maps"” (Eriksson et al. 2001). Latent variables in a statistical meaning are variables that are not manifest - they can not be measured directly. Instead they are computed as linear combinations of a set of manifest variables.

\subsection{Principal Component Analysis (PCA)}

PCA is a frequently used multivariate analysis technique first formulated by Pearson who described the analysis as finding lines and planes of closest fit to systems of points in space (Jackson 1991). It is a projection method designed to extract and display systematic variation in the data matrix $\mathbf{X}$.

The most important use of PCA is to represent a multivariate data table in a lowdimensional space, usually 2 to 4 dimensions or principal components (PC), to provide a "simple" overview of the data. The data overview may reveal groupings of observations, time trends, and outliers. It may also uncover relationships among observations and among variables themselves (Eriksson et al. 2001). "The PCs summarize the systematic patterns of variation between samples. All redundancy (repeated information in the variables and the samples) is thereby summarized" (Martens and Martens 2001). It produces a summary showing: 1) how the observations are related, 2) relationships among variables, 3) which variables contribute similar information to the PCA model, 4) any deviating observations or groups of observations, and 5) a sudden shift in the data or a smooth time series. In essence PCA describes the correlation structure of $\mathbf{X}$ (Eriksson et al. 2001). 
Statistically, PCA finds lines, planes and hyperplanes in the K-dimensional space that approximates the data in the "least squares" sense. The line or plane that approximates the data points makes the variance of coordinates on the line or plane as large as possible while minimizing the variance of the residuals as shown in Figure 3.5.

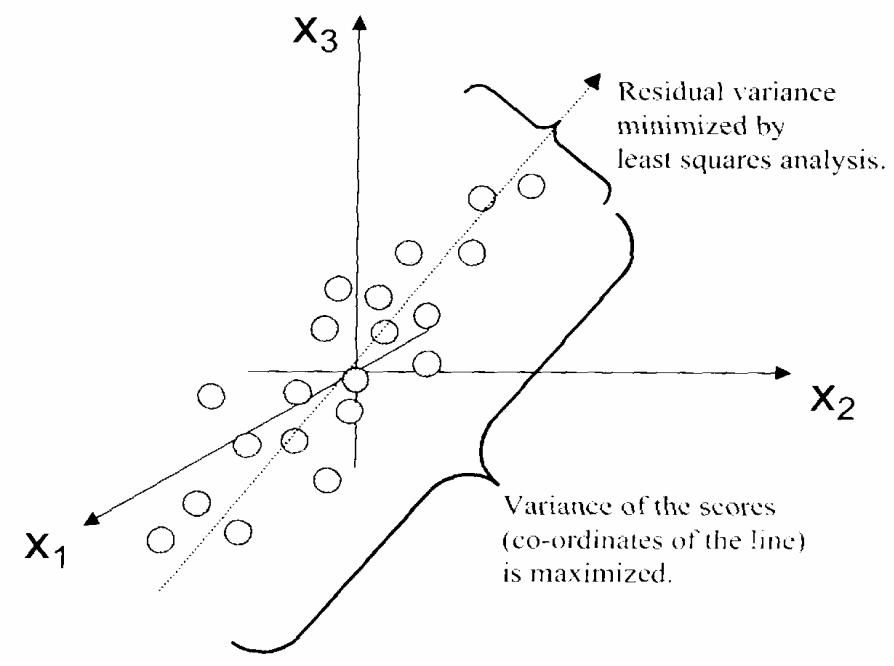

Figure 3.5 PCA derives a model that fits the data in a least squares sense. PCA can be understood as maximizing the variance of the projection coordinates (Eriksson et al. 2001).

PCA is often referred to as a data reduction or summary technique. It is a procedure for removing the redundancy from a set of correlated variables $X=\left\{x_{k}\right.$, $\mathrm{k}=1,2, \ldots, \mathrm{K}\}$ and representing the variables with a smaller set of "derived" variables or latent variables also referred to as principal components, dimensions, or factors, $\mathrm{T}=\left\{\mathrm{t}_{1}, \mathrm{t}_{2}, \ldots \mathrm{t}_{\mathrm{A}}\right\}$ where $\mathrm{A}<\mathrm{K}$. It can be thought of as removing duplicate information from among a set of variables, or, it may be loosely considered as a method of grouping similar variables (Kachigan 1991). It also identifies relationships among variables and "summarizes the systematic patterns of variation between observations" (Martens and 
Martens 2001). It can also serve an inferential role in those instances when the results are generalized to a larger population

The task is to form components that are relatively independent of one another and minimize the effect of collinearity. Variables that are grouped together as one component are more highly correlated with one another than they are with the variables defining other components (Kachigan 1991). The purpose of PCA is to decompose, i.e. break down into component parts, the data matrix $\mathbf{X}$ to detect hidden phenomena, and the concept of variance becomes important. It is a fundamental assumption in PCA that directions in multivariate space with maximum variation are more or less coupled to these hidden phenomena. The principal component latent variables that coincide with these maximum variance directions (Figure 3.5) can be used as an efficient tool to understand and interpret the data (Eriksson et al. 1999).

Kachigan (1991) described several key PCA applications - simplifying data, screening variables, and summarizing data..

Simplifying data is accomplished by identifying the underlying factors or components by grouping the large number of variables into a smaller number of sets and creating a new variable or component that represents each of these sets. This provides better insight into the subject by having fewer variables to study.

Screening variables is possible with PCA because it identifies groupings of variables that are highly correlated with one another. Then a single variable from each group or component can be selected for subsequent analysis thus avoiding the collinearity problem sometimes found with regression analysis. 
PCA can also be used to summarize data, as it has flexibility in its ability to extract as few or as many factors as desired from a set of variables. This allows the data to be represented with few components that account for the bulk of the variance contained in the entire set of variables.

\subsubsection{Overview of Data Analysis Using PCA}

The data analysis begins with a data matrix $\mathbf{X}$, with observations as rows and variables as columns. The observations can be analytical batches, process time points of a continuous process, batches from a batch process, or trials of a design of experiment (DOE). In order to characterize the properties of the observations, variables are measured for each of the observations. The variables might be measurements from sensors in a process (dryer temperatures, resin flows, strand moisture content, etc.). Then a correlation matrix, $\mathbf{X}^{\prime} \mathbf{X}$, is determined for each possible pair of variables. A series of matrix algebra operations using decomposition are performed on the matrix to arrive at a component / factor matrix. In this matrix the columns represent the derived factors or components and the rows represent the original input variables. "The cell entries are called the loadings that vary in value from -1 to +1 and represent the degree to which each of the variables correlates with each of the components. In fact the loadings are nothing more than the correlation coefficients between the original variables and the newly derived components or factors, which are themselves latent variables. An inspection of the loadings will reveal the extent to which each of the variables contributes to the meaning of each of the components. Those variables with high loadings will be the ones that provide the meaning and interpretation of the component" Kachigan (1991). 
Variables with high loadings $(\max =1)$ on a particular component are associated with high weights in the equation for determining component scores. High loading value means the component is aligned with the original variable; while a close to zero value indicates the variable has close to no influence (Umetrics 2003) An object or observation will tend to score high on a component only if it scores high on the variables that load most highly on that component. (Kachigan 1991)

Another way to explain PCA is by the projection method whereby information contained in the mass of process data is projected down on a few "scores". These scores provide a very good summary of process data table, and plot of the scores make it easier to grasp. The coefficients of the projections, i.e., how the variables are combined to form the scores, are called loadings or weights. The loading plots show the importance of the variables, their similarity, their connection, and other things of interest. The parts of the data not seen in the score plots, i.e., the residuals, are displayed, in summarized form, in the DModX plots which are row residual standard deviations. (Eriksson et al. 2001) DModX is the distance to the model in the $\mathrm{X}$-data and indicates how well an observation fits the PCA model.

\subsubsection{A Geometric Interpretation of PCA}

The data table is defined as an $\mathbf{X}$-matrix with $\mathrm{N}$ observations and $\mathrm{K}$ variables. Each observation (each row) of the $\mathbf{X}$-matrix is plotted in the K-dimensional variable space. Each row of the data table becomes a point in the space. Wiith many rows in a data table, a "swarm" of data points is formed in space.

A problem typically encountered with large data tables is the variables may have very different numerical ranges. Since PCA is a maximum variance projection method, a 
variable with a large variance has a greater chance of being included in the model than a variable with a small variance. Preprocessing the data, by unit variance scaling and centering, the variables have a similar possibility of being included in the data analysis.

Unit variance scaling includes determining the standard deviation of the variables (columns) and forming the scaling weights by taking the inverse of each variable's standard deviation. Then each column of $\mathbf{X}$ is multiplied by the inverse of the standard deviation for that column. Basically what has happened is the large varying variables have been shrunk while the small varying variables are stretched. This prevents any one variable from dominating over others because of its numerical range.

Mean centering is conducted by calculating the average value for each of the variables. This results in a vector of averages and is depicted as a point in space. The average values are subtracted from the data which in turn re-positions the coordinate systems so the origin now passes through the average point. The average point can also be thought of as the center of gravity of the data swarm as shown in Figure 3.6. 


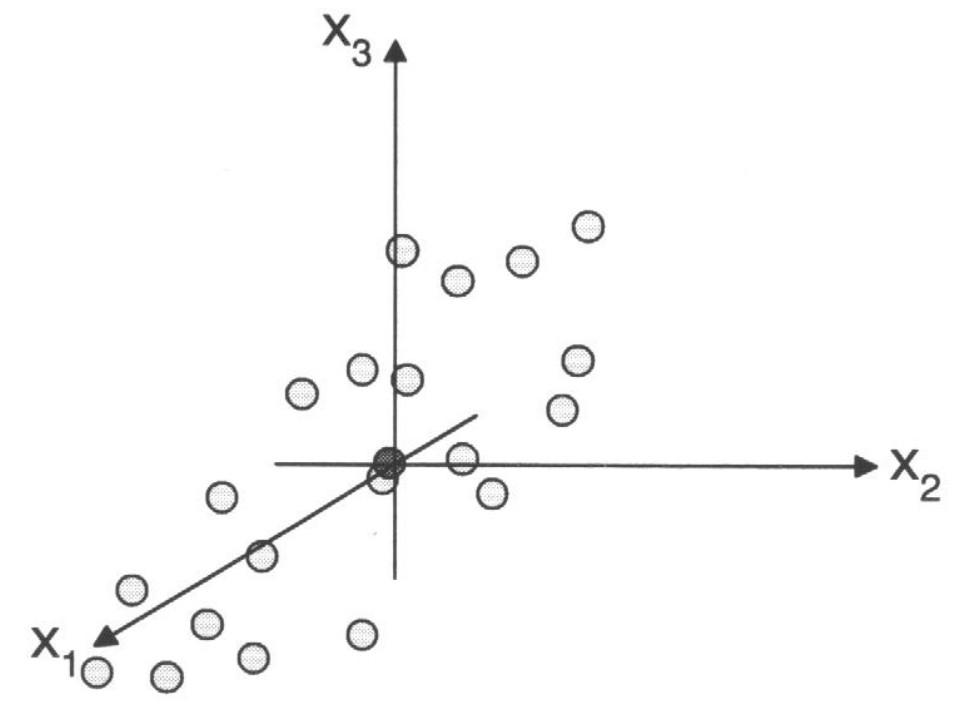

Figure 3.6 Centering data is depicted by moving the average point to the origin of the graph (Eriksson et al. 2001).

Calculating the first principal component can be thought of as the best approximation of the data in the least squares sense that best accounts for the shape of the point swarm. The line is the main component, the maximum variance direction, in the data (Figure 3.5). The line goes through the average point which after mean centering is the origin. Each observation is projected down on the line in order to get a coordinate value along the PC-line. This new coordinate value for each observation is referred to as the score. Usually, one principal component is not sufficient to model the systematic variation of the data. A second principal component is calculated as a straight line in space but it is orthogonal (right angle) to the first PC. This line also passes through the average point and improves the approximation of $\mathbf{X}$. The second line represents the next greatest variance in the data but is orthogonal to the first component.

The two PC lines derived define a plane, or windowpane into the K-dimensional variable space. Each observation is projected to the plane to get the coordinate values 
called scores. A plot of the projected scores is referred to as the score plot. Each observation is now characterized by two values (scores), one along the first PC and the other along the second PC. Those observations that are close together have similar properties, whereas those observations far away from each other are dissimilar. The observations that are close to the center (origin) of the plane indicate they have "average" properties.

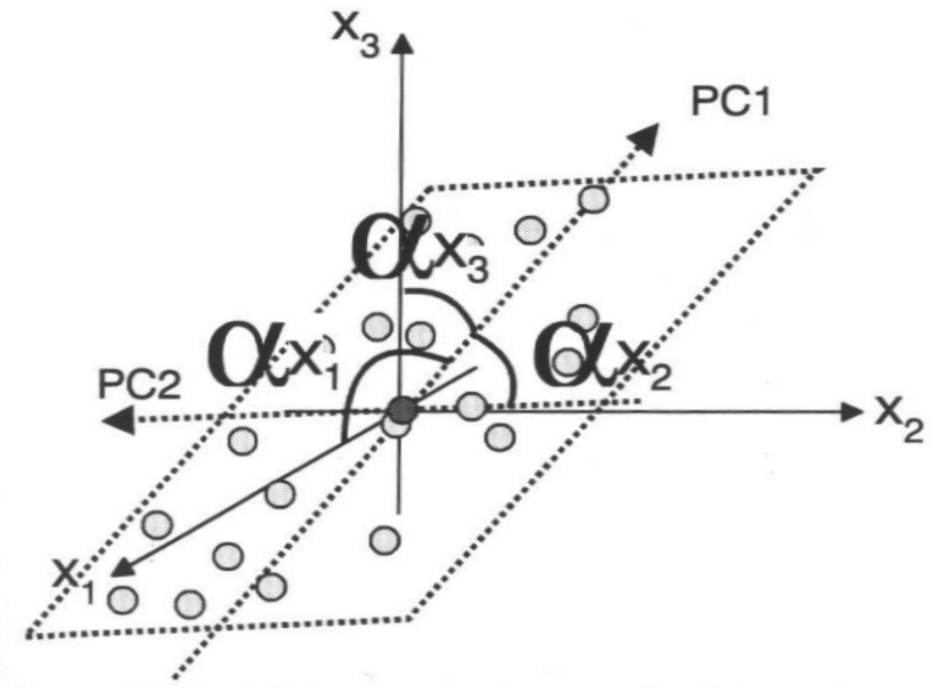

Figure 3.7 The direction of the PC1 in relation to the original variables is given by the cosine of the angles $\alpha_{1}, \alpha_{2}$, and $\alpha_{3}$.

The principal component's loading expresses geometrically the orientation of the model plane in the K-variable space (Figure 3.7). The direction of the $\mathrm{PC} 1$ in relation to the original variables is given by the cosine of the angles $\alpha_{1}, \alpha_{2}$, and $\alpha_{3}$. "These values indicate how the original variables $\mathrm{x}_{1}, \mathrm{x}_{2}$, and $\mathrm{x}_{3}$ 'load' into (=contribute to) PC1. Hence, they are called loadings" (Eriksson, et al. 2001). A second set of loading coefficients indicates the direction of the $\mathrm{PC} 2$ relative to the original variables. With two PC's and three original variables, the six loading values (cosine of angles) are needed to specify the position of the model plane in K-space. 
To interpret a score plot, the loading plot is used to see which variables are responsible for patterns seen in the score plot plus which variables are influential and which ones are correlated. The loading plot is a representation of the variables' loading for the components. Variables contributing similar information are grouped together in the loading plot. The variables that are negatively correlated are usually positioned on opposite sides of the plot origin and in diagonally opposed quadrants. The distance to the origin also provides information. The further away from the origin the variable lies, the stronger the influence that variable has on the model.

To compute a third PC, the PC must be (1) oriented in the direction of the third largest variation in the data swarm, (2) orthogonal to the first two PC's and (3) pass through the average point (origin). The scores and the loading values can then be plotted and reviewed as discussed above.

\subsubsection{Mathematical Interpretation of PCA}

Mathematically there are several numerical algorithms that lead to the same resulting PCA solution. In some of the algorithms, it is normal to compute all non-zero eigenvalues and their eigenvectors simultaneously, either from the data centered $\mathbf{X}$ table or via the $\mathbf{X}^{\prime} \mathbf{X}$ cross product matrix. However, working with the data table $\mathbf{X}$ is considered easier than going via the more abstract cross-products table.

The NIPALS (Nonlinear Iterative Partial Least Square) algorithm extracts one factor at a time. It computes the largest eigenvalues with the most important eigenvectors using the fact that the principal components are orthogonal both in scores and loadings to extract one single factor or component at a time, $a=1, a=2, \ldots$, A. NIPALS employs for each factor an iterative method to obtain the loading vector $p_{a}$ and the score vector, $t_{a}$, 
from the residual matrix obtained after estimation of the previous a- 1 factors. Martens and Naes (1989) call the preliminary residual matrix $\mathbf{X}_{\mathrm{a}-1}$ to make it easier to describe the algorithm. Other authors refer to it as $\hat{\mathbf{E}}$. Starting with some guessed scores, the iteration occurs as follows: "The loading estimate is improved by regression of $\mathbf{X}_{\mathrm{a}-1}$ on the previous score estimate, and the score estimate is improved by regression of $\mathbf{X}_{\mathrm{a}-1}$ on the improved loading estimate, and so on until convergence” (Martens and Naes 1989).

Martens and Naes (1989) describe the basic steps in the NIPALS algorithm. The algorithm extracts one factor at a time with each factor obtained iteratively by repeated regressions of $\mathbf{X}$ on the scores, $\hat{\mathbf{t}}$, to obtain improved $\hat{\mathbf{p}}$ and regressions of $\mathbf{X}$ on these $\hat{\mathbf{p}}$ to obtain improved $\hat{\mathbf{t}}$. The algorithm proceeds as follows.

Pre-scale the X-variables to ensure comparable noise levels. Then center the $\mathrm{X}$-variables by subtracting the mean, $\overline{\mathbf{x}}^{\prime}$, forming $\mathbf{X}_{\mathbf{0}}$. Then for factors $\mathrm{a}=1,2 \ldots \mathrm{A}$, compute $\hat{\mathbf{t}}_{\mathbf{a}}$ and $\hat{\mathbf{p}}_{a}$ from $\mathbf{X}_{\mathbf{a}-\mathbf{1}}$.

Start: Select start values, e.g. $\hat{\mathbf{t}}_{\mathbf{a}}=$ column in $\mathbf{X}_{\mathrm{a}-1}$ that has the highest remaining sum of squares. Then repeat the following steps until convergence.

i. Improve estimate of loading vector $\hat{\mathbf{p}}_{\mathbf{a}}$ for this factor by projecting the matrix $\mathbf{X}_{\mathrm{a}-1}$ on $\hat{\mathbf{t}}_{\mathrm{a}}$, i.e.

$$
\mathbf{p}_{\mathbf{a}}^{\prime}=\left(\hat{\mathbf{t}}_{\mathbf{a}}^{\prime} \hat{\mathbf{t}}_{\mathbf{a}}\right)^{-1} \hat{\mathbf{t}}_{\mathbf{a}}^{\prime} \mathbf{X}_{\mathbf{a}-\mathbf{1}}
$$

ii. Scale length of $\hat{\mathbf{p}}_{\mathbf{a}}$ to 1.0 to avoid scaling ambiguity:

$$
\hat{\mathbf{p}}_{\mathbf{a}}=\hat{\mathbf{p}}_{\mathbf{a}}\left(\hat{\mathbf{p}}_{\mathbf{a}}{ }^{\prime} \hat{\mathbf{p}}_{\mathbf{a}}\right)^{-0.5}
$$

iii. Improve estimate of score $\hat{\mathbf{t}}_{\mathrm{a}}$ for this factor by projecting the matrix $\mathbf{X}_{\mathrm{a}-1}$ on $\hat{\mathbf{p}}_{\mathrm{a}}$ : 


$$
\hat{\mathbf{p}}_{\mathbf{a}}=\hat{\mathbf{p}}_{\mathbf{a}}\left(\hat{\mathbf{p}}_{\mathbf{a}}^{\prime} \hat{\mathbf{p}}_{\mathbf{a}}\right)^{-0.5}
$$

iii. Improve estimate of score $\hat{\mathbf{t}}_{\mathbf{a}}$ for this factor by projecting the matrix $\mathbf{X}_{\mathrm{a}-1}$ on $\hat{\mathbf{p}}_{\mathbf{a}}$ :

$$
\hat{\mathbf{t}}_{\mathrm{a}}=\mathbf{X}_{\mathrm{a}-1} \hat{\mathbf{p}}_{\mathrm{a}}\left(\hat{\mathbf{p}}_{\mathrm{a}}{ }^{\prime} \hat{\mathbf{p}}_{\mathrm{a}}\right)^{-1}
$$

iv. Improve estimate of the eigenvalue $\hat{\boldsymbol{\tau}}_{\alpha}$ :

$$
\hat{\boldsymbol{\tau}}_{\mathrm{a}}=\hat{\boldsymbol{\tau}}_{\mathrm{a}}^{\prime} \hat{\boldsymbol{\tau}}_{\mathrm{a}} \quad \text { where: } \hat{\boldsymbol{\tau}}_{\mathrm{a}}=t_{\mathrm{a}}^{\prime} t_{\mathrm{a}}
$$

v. Check convergence: If $\hat{\boldsymbol{\tau}}_{\boldsymbol{\alpha}}$ minus $\hat{\boldsymbol{\tau}}_{\boldsymbol{\alpha}}$ in the previous iteration is smaller than a certain amount pre-specified constant, e.g. 0.00001 times $\hat{\boldsymbol{\tau}}_{\alpha}$, the method has converged for this factor. If not, go to step i. If converged, subtract the effect of this factor:

$$
\mathbf{X}_{\mathrm{a}}=\mathbf{X}_{\mathrm{a}-\mathbf{1}}-\hat{\mathbf{t}}_{\mathrm{a}} \hat{\mathbf{p}}_{\mathrm{a}}{ }^{\prime}
$$

and go to start for the next factor.

More formally the loading $\hat{\mathbf{p}}_{1}$ is defined as the normalized (length=1) vector that maximizes the empirical variance of $\hat{\mathbf{p}}_{\mathbf{1}}{ }^{\prime} \mathbf{x}$ or in other words maximizes $\hat{\mathbf{p}}_{\mathbf{1}}{ }^{\prime} \mathbf{X}^{\prime} \mathbf{X} \hat{\mathbf{p}}_{\mathbf{1}}=\hat{\mathbf{t}}_{\mathbf{1}}^{\prime} \hat{\mathbf{t}}_{\mathbf{1}}$. The next factor's loading, $\hat{\mathbf{p}}_{2}$ is defined as the vector maximizing the same quantity, i.e. the scalar $\hat{\mathbf{p}}_{2}{ }^{\prime} \mathbf{X}^{\prime} \mathbf{X} \hat{\mathbf{p}}_{2}=\hat{\mathbf{t}}_{2}^{\prime} \hat{\mathbf{t}}_{2}$ under the constraint that the $\hat{\mathbf{t}}_{\mathbf{1}}$ and $\hat{\mathbf{t}}_{\mathbf{2}}$ are orthogonal, i.e. $\hat{\mathbf{t}}_{1}^{\prime} \hat{\mathbf{t}}_{\mathbf{2}}=0$. The procedure continues under the constraint that new factors' scores are uncorrelated or orthogonal with those of the previous factors.

The eigenvalues, denoted as $\hat{\boldsymbol{\tau}}_{\mathbf{1}} \cdot \hat{\boldsymbol{\tau}}_{\mathbf{a}} \ldots \hat{\boldsymbol{\tau}}_{\mathbf{K}}$, show how much variability each component removes from $\mathbf{X}$. The sum of squares of the principal component scores, $\hat{\mathbf{t}}_{\mathbf{1}} \ldots \hat{\mathbf{t}}_{\mathbf{k}}$ are computed as $\boldsymbol{\tau}_{a}=\mathbf{t}_{\mathbf{a}}^{\prime} \mathbf{t}_{\mathbf{a}}$ where $\mathrm{a}=1,2, \ldots \mathrm{k}$. 
For centered $\mathrm{X}$-variables, it can be shown that the orthogonal loading vectors $\hat{\mathbf{p}}_{\mathrm{a}}$ where $\mathrm{a}=1.2 \ldots \mathrm{A}$ are eigenvectors of $\mathbf{X}^{\prime} \mathbf{X}$ with the $\hat{\boldsymbol{\tau}}^{\prime} \mathrm{s}$ as eigenvalues. This means that all $\hat{\mathbf{p}}^{\prime}$ s satisfy the equation $\mathbf{X}^{\prime} \mathbf{X} \mathbf{p}_{\mathbf{a}}=\mathbf{p}_{\mathrm{a}} \boldsymbol{\tau}_{\mathbf{a}}$. Likewise it can be shown that the scores $\hat{\mathbf{t}}_{\mathbf{a}}$, $\mathrm{a}=1,2, \ldots$ A represent the corresponding eigenvectors of $\mathbf{X}^{\prime} \mathbf{X}$ scaled to length $\sqrt{\hat{\boldsymbol{\tau}}_{\mathrm{a}}}$.

If all the $\mathrm{A}=\mathrm{K}$ eigenvectors have been extracted with some having eigenvalues of or near zero, then $\mathbf{X}$ can be written as $\mathbf{X}=\hat{\mathbf{T}} \hat{\mathbf{P}}^{\prime}$. Scaling the principal component scores for each component to length one and denoting the resulting matrix $\mathbf{U}$, the $\mathbf{X}$ matrix can be written as $\hat{\mathbf{U}} \operatorname{diag}\left(\sqrt{\hat{\boldsymbol{\tau}}_{a}}\right) \hat{\mathbf{P}}_{\mathrm{a}}$ which is exactly the singular value decomposition of X (Martens and Naes 1989).

The component loading values and the weights for determining the component scores are derived more or less simultaneously in such a way that there is little or no correlation between resulting components, that is so they are independent of each other. It follows then those variables that are highly correlated with each other form one component, while those variables that are not correlated with each other will form separate components. It is on this basis that PCA is able to remove redundancy from a set of variables (Kachigan 1991).

PCA can model the data table $\mathbf{X}$ as:

$$
X=1 * \overline{x^{\prime}}+T * P+E
$$

Where: $1{ }^{*} \bar{x}^{\prime}$ represents the variable averages and comes from the data preprocessing step.

$\mathbf{T} * \mathbf{P}$ ' is a matrix product which models the data structure.

$\mathbf{T}$ is the score matrix. (M x A) 


$$
\begin{aligned}
& \mathbf{P} \text { is the loading matrix }(\mathrm{A} \times \mathrm{K}) \\
& \mathbf{E} \text { is the residual matrix. (M x K) }
\end{aligned}
$$

\subsubsection{PCA Model Interpretation}

An interpretation of a PCA model begins with a review of the observations by looking for outliers in the data set. PCA uncovers outliers, both moderate and strong in the data set. Outliers are theoretically defined as observations that do not fit the PCAmodel. But outliers may be very informative and not at all erroneous. "The observation may be an outlier because it alone spans a certain type of important variability in the Xdata" (Martens and Naes 1989). One should be aware of outliers but what do with them depends on the person developing the model. Strong outliers are found in reviewing the PC score plots; while moderate outliers are determined when inspecting model residuals using DModX. Model residuals are the deviations of the real data from the model or the X-variation not captured by the PC model.

The strong outliers, found in the score plots, have leverage to pull the PC-model toward them and may "consume" one component just because of their existence. A diagnostic showing these outliers is given by Hotelling's $T^{2}$. This statistic is the multivariate equivalent of Student's t-test and provides a check for observations adhering to multivariate normality. When used in conjunction with the score plot, Hotelling's $\mathrm{T}^{2}$ defines the normal area corresponding to the $95 \%$ confidence region. It should be noted that $\mathrm{N}^{*} 0.05$ observations will be found outside the $95 \%$ confidence region, and only a handful of these "potential" outliers will be "real" outliers. The outlier is the distance the point is from the center of the model. 
If outliers are found the question arises, should it be removed from the data set? This is up to the experimenter. To aid in making the decision, a contribution plot should be studied to determine the reason for the observation is an outlier. Variables that had the greatest contributions to the score, that is had the highest and lowest values, should be reviewed. It is still paramount that the experimenter uses their scientific expertise to make the call. It is sometimes found that a transcription error is the cause for the outlier.

The moderate outliers while not strong enough to shift the model plane will show up as residuals. The detection tool for identifying moderate outliers is a called distance to the model in X-space or DModX. It is the distance the point is from model plane. It is based on the residual matrix $\mathbf{E}$ and summarizes the elements row by row or for each observation. These values are then plotted in a control chart where the maximum tolerable distance (D-Crit) is given. With process data, moderate outliers are often seen with temporary process upsets, but sometimes a process shift may be discovered by uncovering a persistent high occurrence of outliers.

$$
\text { DModX is calculated as } s_{i}=\sqrt{\frac{\sum e_{i k}^{2}}{K-A}} * v \text {. }
$$

The summation is made over the $\mathrm{X}$-variables and $\mathrm{e}_{\mathrm{ik}}$ are the $\mathrm{X}$-residuals of observation $\mathrm{i}$. $v$ is a correction factor that is a function of the number of observations and the number of components and is slightly larger than one (Eriksson et al. 2001).

The PCA model interpretation next considers the variables in the data set to determine to what extent each variable is accountable for the model. This is accomplished by reviewing the explained variation of each variable. A quantity that ranges from 0 (no explanation) to 1 (complete explanation). By the column wise summation of the residual elements of $\mathbf{E}$, it is possible to describe how well a variable is modeled by the calculation 
of the explained variation $\left(\mathrm{R}^{2}\right)$. The values of $\mathrm{R}_{\mathrm{k}}{ }^{2}$ are related to the loadings and for each component, $\mathrm{a}, \mathrm{p}_{\mathrm{ak}}{ }^{2}$ is proportional to how much the $\mathrm{k}^{\text {th }}$ variable is modeled by this component. (Eriksson et al. 2001)

The next step is determining how many components should be included in the model. It is considered prudent to use as few components as possible in the model to explain or predict the response of interest. The method used to determine the number of components is the difference between the goodness of fit, $\mathrm{R}^{2}$, and the predictive ability of the model, $\mathrm{Q}^{2}$. The predictive ability of the model, $\mathrm{Q}^{2}$, is more important than goodness of fit in model development. The problem with goodness of fit is that with too many variables and components in the model, $\mathrm{R}^{2}$ can be made to approach the maximum value of one (1.0). The predictive ability, $\mathrm{Q}^{2}$, on the other hand, is less inflationary and will not automatically come close to 1 with increasing model complexity.

$$
\begin{aligned}
& \mathrm{R}^{2}=1-\mathrm{RSS} / \mathrm{SSX}_{\text {tot.corr }} \\
& \mathrm{Q}^{2}=1 \text {-PRESS } / \mathrm{SSX}_{\text {tot.corr }}
\end{aligned}
$$

Where: RSS is the residual sum of squares,

PRESS is the predictive residual sum of square, which is the summed squared difference between predicted and observed values, and $\mathrm{SSX}_{\text {tot.corr }}$ represents the total variation in the $\mathbf{X}$ matrix after mean centering.

In evaluating $\mathrm{R}^{2}$ and $\mathrm{Q}^{2}$, it is generally believed a $\mathrm{Q}^{2}>0.5$ is regarded as good and a $\mathrm{Q}^{2}>0.9$ is excellent. It is also important that the difference between $\mathrm{R}^{2}$ and $\mathrm{Q}^{2}$ must not be large and preferably should not exceed 0.2 to 0.3 (Eriksson et al. 2001). 
Cross validation $(\mathrm{CV})$ is used to find the optimal model dimensionality or number of components and is used to estimate the predicative ability of the model with increasing number of components. The basic idea is to hold a portion of the data out of the model development, develop a number of parallel models from the reduced data, predict the omitted data using the different models, and finally compare the predicted values with the actual ones. The squared differences between predicted and observed values are summed to form the predictive residual sum of squares (PRESS) which is a measure of the predictive power of the tested model. The model building continues adding an additional dimension, a, and the PRESS is compared with the residual sum of squares of the previous dimension. When the PRESS is not significantly smaller than residual sum of squares (RSS), the tested dimension is considered insignificant and the model building is stopped.

$\mathrm{Q}^{2}$ is a reasonable first guess on how well the model will perform on new data but the real test is to use an external validation set.

A valid model can be defined as one that predicts much better than chance. It should also have model parameters with little bias, have correct sign and be large for important variables and small for unimportant variables. Finally it should be consistent with fundamental scientific and technical knowledge.

\subsection{Projection to Latent Structures By Means of Partial Least Square}

Projection to Latent Structures by means of Partial Least Square (PLS) may be thought of as the regression extension of PCA.

Eriksson et al. (1999) described PLS as "a technique of generalized regression to model the association between $\mathrm{X}$ and $\mathrm{Y}$, as well as a philosophy of how to deal with 
complicated and approximate relationship. It uses the regularities in the data themselves as building blocks for the models, instead of hypothetical functional forms derived from an underlying theory. In this way projection models like PLS function in cases where no good first principles theory exists, and also provide an independent check on the validity of models derived from the theory existing in the field of the ongoing application".

Martens and Naes (1998) refer to PLS as Partial Least Squares and "is a loose term for a family of philosophically and technically related multivariate methods derived from Herman Wold's basic concept of iteratively fitting of bilinear models in several blocks of variables." It was developed around 1975 by Herman Wold as a method for relating two data matrices, $\mathbf{X}$ and $\mathbf{Y}$, to each other by a linear multivariate model. It derived its usefulness from its ability to analyze data with many noisy, collinear, and even incomplete variables in both $\mathbf{X}$ and $\mathbf{Y}$.

Eriksson et al. (1999) describes PLS as a technique that model, "complicated data sets in terms of chains of matrices, so called path models. Each model parameter is iteratively estimated as the slope of a simple bivariate regression (least squares) between a matrix column or row as the $\mathrm{y}$-variable, and another parameter as the $\mathrm{x}$-variable. So, for instance, in each iteration the PLS weights, w, are re-estimated as $\mathbf{u}^{\prime} \mathbf{X} /\left(\mathbf{u}^{\prime} \mathbf{u}\right)$. The partial in PLS indicates that this is a partial regression since the second parameter vector $(\mathbf{u})$ is considered as fixed in the estimation. This partial least squares interpretation shows that we can see any matrix-vector multiplication as equivalent to a set of bivariate regressions. This provides an intriguing connection between two central operations in matrix algebra and statistics" (Eriksson et al. 1999). 
PLS forms "new X-variables", $t_{a}$, as linear combinations of the old ones, and thereafter uses these new T's as predictors of $\mathbf{Y}$. The number of new $\mathbf{t}^{\prime}$ 's (components) is determined using cross validation to determine predicting capability.

The aim of PLS is "to extract the main variation patterns from one data table $\mathbf{X}$ that have relevance also for another data table $\mathbf{Y}$ from the same samples. This allows interpreting the structures within and between $\mathbf{X}$ and $\mathbf{Y} "$ Martens and Martens (2001). PLS models, "complex process data with an aim to accomplish fast, accurate, and quantitative predictions of complex responses based on the collected body of X-data" (Eriksson et al. 2001). PLS has been developed explicitly for this type of situation with numerous, often-correlated input and process variables and result variables. PLS is also seen as "a philosophy of how to deal with complicated and approximate relationships" (Eriksson et al. 1999).

In process modeling, PLS is used to find relationships between variables measured on the process $(\mathrm{X})$ at $\mathrm{N}$ time points and corresponding values of "result variables" (Y) such as product properties, e.g. moisture content and strength. Some of the variables in the database are specified as predictor variables $(\mathbf{X})$, and some of the variables are specified as dependent or response variables (Y). PLS finds the relation between the two groups of variables. Eriksson, et al. (2001) reported the precision of the PLS model improves with increasing number of relevant X-variables.

PLS modeling consists of simultaneous projections of both $\mathbf{X}$ and $\mathbf{Y}$ spaces on low dimensional hyperplanes. The coordinates of the points on these hyperplanes constitute the elements of the matrices $\mathbf{T}$ and $\mathbf{U}$. The analysis has the following 
objectives: to approximate the $\mathrm{X}$ and $\mathrm{Y}$ spaces and to maximize the correlation between $\mathrm{X}$ and $\mathrm{Y}$

With PLS it is possible to find out how the factors influence the responses, how the responses correlate with each other, and how to adjust the factors to get the desired responses. In addition PLS allows multiple responses (Y) to be incorporated in one regression model. This is possible as long as the responses are reasonably well correlated and thus give similar information about the observations. The strong correlation of $\mathbf{Y}$ has a tendency to stabilize the model.

It should be noted that if the responses are really measures of different things and are independent, little is gained by analyzing them in the same model. A PLS model with non-correlated Y-variables tends to have many components and is difficult to interpret. Separate models for the Y's gives simpler models that have fewer dimensions and are easier to interpret. Eriksson et al. (1999) states "a reasonable cut off for "strong" correlation seems to be around 0.5." To judge whether Y's are correlated or not it is generally recommended starting the analysis with a PCA of the Y-matrix. If the number of components of the PC model is small compared to the number of Y-variables and the components can be understood, then it can be concluded the Y's are correlated and a PLS model of all the Y's is warranted. If there are many components and the Y's are not correlated, it is best to model the Y's in separate PLS models.

The difference between PLS and PCA is the later is a maximum variance projection of $\mathbf{X}$, whereas the former is a maximum covariance model of the relationship between $\mathbf{X}$ and $\mathbf{Y}$. As with PCA, data are pre-processed with data centered and scaled to unit variance prior to using PLS. With PLS a variable may be unduly influential on the 
model parameters, and this influence will increase with the variance of the variable. Scaling all variables to unit variance assumes all variables are equally important, a priori (Eriksson et al. 1999).

\subsubsection{Geometric Interpretation of PLS}

\subsubsection{Single Response $(M=1)$}

A geometric interpretation of PLS with one response $(\mathrm{M}=1)$ will be discussed before exploring multiple response models $(M>1)$. As with PCA, each observation can be represented graphically; however with PLS, each data row corresponds to two points rather than one, one in the $\mathrm{X}$-space and one in the Y-space. Therefore, two data swarms are created in these spaces. The question then is to find whether there is a relationship that exists between the way the observations are grouped in the predictor X-space and the way they are spread in the response Y-space.

After plotting the observations in their spaces, the next step is to calculate the first component. This initial component can be interpreted as inserting a line in the X-space. This line passes through the origin and is fitted to approximate the $\mathbf{X}$ data swarm plus provides a good correlation with the Y-variable. By projecting the sample to the line, the coordinate of an $\mathbf{X}$ observation is obtained. This coordinate is the score, $\mathrm{t}_{\mathrm{i} 1}$, of observation $i$. The scores of all the observations form the first $\mathrm{X}$-score vector $\mathrm{t}_{1}$. This vector can be thought of as a new variable, a latent variable, which reflects "only the information in the original $\mathrm{X}$-variables that is of relevance for modeling and predicting the response $\mathrm{Y}$-variable. This score can be used to acquire an estimate of $\mathbf{y}, \hat{\mathbf{y}}_{1}$, after the 
first PLS component which is accomplished by multiplying $t_{1}$ by the weight of the $y$-data, $\mathrm{c}_{1}$.

The differences between $\mathbf{y}-\hat{\mathbf{y}}_{1}$, are called residuals that represent the variation left unexplained by the first PLS component. For an ideal model there would be small or no residuals at all. The residuals can be looked at as a residual vector $\mathbf{f}_{\mathbf{1}}$ and will be shorter than the parent vector of observed y-data as the first component removes variation. Figure 3.8 depicts the residual vector $\mathbf{f}_{\mathbf{1}}$ on the right side of the figure.
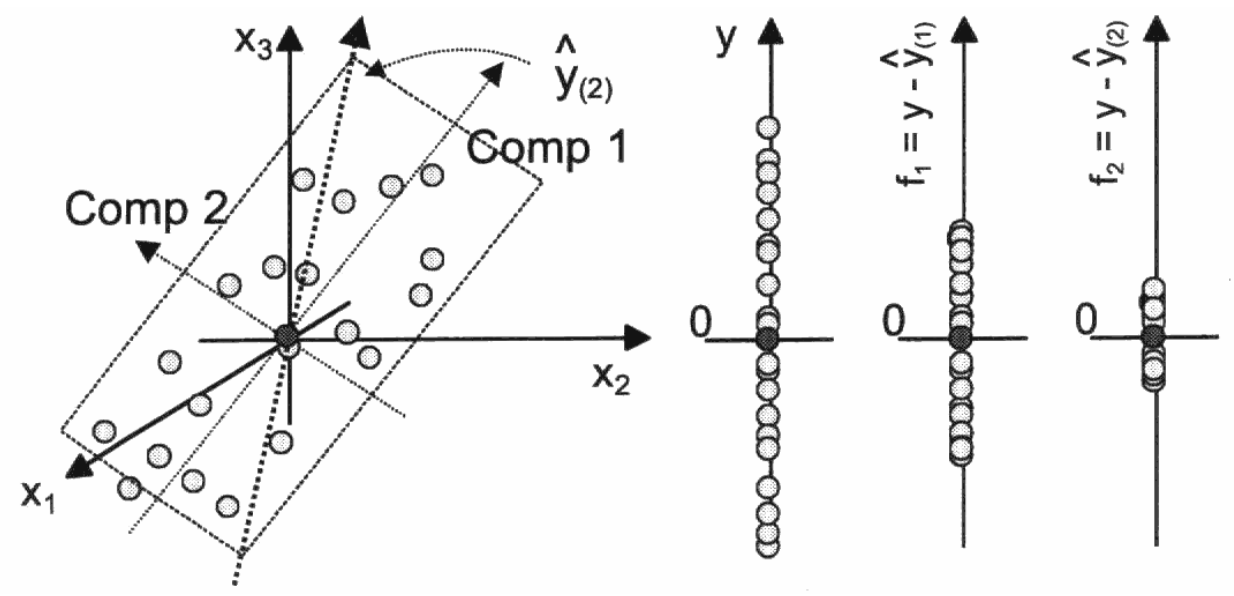

Figure 3.8 The PLS model $(\mathrm{m}=1)$ is depicted as reducing the variation in the variable as the components are determined in the left portion of the figure the residuals $f$ values are reduced as the variation is removed by the components as shown in the right portion of the figure (Eriksson et al. 2001)

Usually one component is inadequate to describe the variation in the y-data. By adding a second component, it is possible to improve the descriptive ability of the PLS model. The second component can also be interpreted as a line in X-space that passes through the origin but is orthogonal to the first component. This component finds the direction in X-space that improves the description of the $\mathrm{X}$-data as well as provides a good correlation with the y-residuals, $f_{1}$, remaining after the subtraction of the first component. 
In order to obtain the score values of the observations along the second projection coordinate in the X-space, all observations are projected to the second component line. This gives the score vector, $t_{2}$. Then $t_{2}$ is multiplied times the second weight of the $\mathrm{Y}$ data, $\mathrm{c}_{2}$, to show the correlation with the y-residual $\mathrm{f}_{2}$, after the first dimension. An estimate of $y$ after two model components, $\hat{\mathbf{y}}_{\mathbf{2}}$, is obtained by computing $\mathbf{c}_{\mathbf{1}} \mathbf{t}_{\mathbf{1}}+\mathbf{c}_{\mathbf{2}} \mathbf{t}_{\mathbf{2}}$. Geometrically this more precise estimate of $y, \hat{\mathbf{y}}_{2}$ is interpretable as the resultant of the vector addition of component 1 and component 2 in the X-space.

Figure 3.8 helps to demonstrate the power of the PLS model. The $\hat{\mathbf{y}}_{2}$ is geometrically interpretable, in the left hand side of the figure, as the resultant vector from the vector addition of component 1 and component 2 in $\mathrm{X}$-space. In the right hand side of the figure, the first component has explained a lot of the y-variation and only a small portion remains in the residual $f_{1}$. The situation is further improved after the second component, as the residual $\mathrm{f}_{2}$ is smaller than $\mathrm{f}_{1}$.

\subsubsection{Multiple Response $(M>1)$}

How does PLS operate when the response data comprise a matrix $\mathbf{Y}$ rather than a vector $\mathrm{y}$ ? Consider a case in which there are five $\mathrm{X}$-variables $(\mathrm{K}=5)$ and three $\mathrm{Y}$-variables $(\mathrm{M}=3)$. For each matrix it is possible to construct a space with $\mathrm{K}$ and $\mathrm{M}$ dimensions. In these two spaces, each $\mathrm{X}$ - and $\mathrm{Y}$-variable represent a coordinate axis with a length defined by its scaling that, in most cases, is scaled to unit variance. Each observation in a data set is both one point in the $\mathbf{X}$-space and another point in the $\mathbf{Y}$-space. Thus with many observations, point swarms are formed in the $\mathbf{X}$ - and $\mathbf{Y}$-spaces. The two point-

swarms have shapes with a unique distribution of points inside each cluster. The 
relationship between the way the observations are grouped in the predictor $\mathbf{X}$-space and the way they spread in the response Y-space can be determined by PLS.

The first PLS component is fitted to the data by inserting one line in the X-space and another in the Y-space. The lines are determined as the best approximation of the point swarms in $\mathrm{X}$ and $\mathrm{Y}$ as well as providing a good correlation between $\mathrm{X}$ and $\mathrm{Y}$. The two lines intersect with their average values since the data is normally mean centered and unit scaled during pre-processing of the data. By projecting the observations to the two lines, the scores $t_{1}$ and $\mathrm{u}_{1}$ are obtained for $\mathbf{X}$ and $\mathbf{Y}$ respectively.

The correlation between $\mathbf{X}$ and $\mathbf{Y}$, in terms of the two score vectors $t_{1}$ and $\mathrm{u}_{1}$, may be checked graphically in a scatter plot called the score plot. This is possible because the two score vectors are connected by the inner relationship $\mathrm{u}_{i 1}=\mathrm{t}_{\mathrm{i} 1}+\mathrm{h}_{\mathrm{i}}$ where $\mathrm{h}_{\mathrm{i}}$ is a residual. If the correlation is strong then the spread of points will be narrow or tight; conversely when the correlation is weak there is considerable spread of points in the scatter plot. The score plot also makes it possible to discover outliers in the $\mathbf{X}$-data, $\mathbf{Y}$-data and outliers in the relationship between $\mathbf{X}$ and $\mathbf{Y}$. In addition, it is possible to detect with the score plot non-linearity between the predictors and the responses. In short, the scores $t$ and $u$ contain information about the observations and their similarities / dissimilarities with respect to the given problem and model.

The second PLS component can be added to the model when dealing with several responses by inserting a second line in each space. The second line in the $\mathbf{X}$-space is orthogonal to the first one, whereas in the $\mathbf{Y}$-space this may not necessarily be the case. These second lines improve the approximation and correlation of $\mathbf{X}$ and $\mathbf{Y}$ as much as possible. Geometrically, a two-component PLS model can be interpreted as spanning 
planes in the $\mathbf{X}$ - and $\mathbf{Y}$-spaces. By projecting the observations to these planes, the PLS scores $\mathbf{t}_{\mathbf{1}}$ and $\mathbf{t}_{\mathbf{2}}$ in $\mathbf{X}$ and $\mathbf{u}_{\mathbf{1}}$ and $\mathbf{u}_{\mathbf{2}}$ in $\mathbf{Y}$ are obtained.

Like with the first score vector pair $\left(\mathbf{t}_{1} / \mathbf{u}_{1}\right)$, plotting the second score vector pair $\left(\mathbf{t}_{2} / \mathbf{u}_{2}\right)$ enables the visualization of the correlation structure. Normally the score vectors of the second PLS component correlate less well than the first pair of latent variables. This is quite logical as the first component captures the strongest source of variation in the data, the strongest signal. After removing the variation that is accounted for by the first component, weaker signals remain in the data and therefore correlation between $\mathbf{X}$ and $\mathbf{Y}$ (in terms of $\mathbf{t}_{\mathbf{1}}$ and $\mathbf{u}_{\mathbf{1}}$ ) is usually weaker and less distinct.

Once a PLS model is established, interpretation of its meaning is important. Model interpretation looks at the PLS model parameters call weights. These weights are the same as the PCA loadings and are denoted as $\mathbf{~ W}^{*}$ and $\mathbf{c}$ for the $\mathbf{X}$ and $\mathbf{Y}$ variables respectively. The weights give information about how variables combine to form the quantitative relation between $\mathbf{X}$ and $\mathbf{Y}$. The weights are essential for understanding which $\mathbf{X}$-variables are important (numerically large $\mathbf{w}$-values) and which $\mathbf{X}$-variables provide the same information for different Y-variables (similar profiles of $\mathbf{w}_{\mathbf{a}}$-values) and for interpreting the scores, $\mathbf{t}$ 's.

The weights show which variables contribute to the model and which variables are not modeled at all. In principle, this means that the PLS weights reflect the relationships among all variables at the same time, and tell which are associated and which contribute unique information. Thus with PLS one obtains information on what $\boldsymbol{X}$ gives $\boldsymbol{Y}$, or how to "set" $\boldsymbol{X}$ to get a desired $\boldsymbol{Y}$. "This implies that in process modeling it might not only be possible to understand the mechanism of the process, but also to extract 
clues of how to modify the regulation pattern of the process to get enhanced product quality and production economy" (Eriksson et al. 1999). It might be possible to uncover which combination of factors ( $\boldsymbol{X}$-variables) give optimal production settings for producing high quality products based on the $\mathbf{Y}$-variables of study.

PLS might be best explained by looking at the end purpose of PLS modeling which is to predict $\mathbf{Y}$ from $\mathbf{X}$, according to:

$$
\begin{aligned}
& \mathbf{X}=\mathbf{1} \overline{\mathbf{x}}^{\mathbf{}}+\mathbf{T} \mathbf{P}^{\mathbf{\prime}}+\mathbf{E} \text { and } \\
& \mathbf{Y}=\mathbf{1} \overline{\mathbf{y}}^{\mathbf{\prime}}+\mathbf{U} \mathbf{C}^{\mathbf{\prime}}+\mathbf{F}\left(=\mathbf{1} \overline{\mathbf{y}}^{\mathbf{\prime}}+\mathbf{T} \mathbf{C}^{\mathbf{\prime}}+\mathbf{G}, \text { due to inner relation }\right) \\
& \mathbf{U}=\mathbf{T}+\mathbf{H} \text { (the inner relation) }
\end{aligned}
$$

In the expressions $1 \overline{\mathbf{x}}^{\prime}$ and $\mathbf{1} \overline{\mathbf{y}}^{\prime}$, represent the variable averages and originate from the data pre-processing step. The information related to the observations are stored in the PLS score matrices $\mathbf{T}$ and $\mathbf{U}$, and the variable related information are in the $\mathbf{X}$-loading matrix $\mathbf{P}^{\prime}$ and $\mathbf{Y}$-weight matrix $\mathbf{C}^{\prime}$. The variation in the data not explained by the model form the residual matrices, $\mathbf{E}, \mathbf{F}$, and $\mathbf{H}$.

Figure 3.9 shows all the matrices that PLS uses or creates. There is an X-weight matrix, $\mathbf{W}$, although it is not defined in the equations above. The $\mathbf{W}$, weights, expresses the correlation between $\mathbf{U}$ and $\mathbf{X}$ and is used to calculate $\mathbf{T}$. $\mathbf{W}$ contains the $\mathrm{X}$-weight vector $\mathbf{w}_{\mathbf{a}}$, which shows how the original variables are linearly combined to form score vectors, $\mathbf{t}_{\mathbf{a}}$. By using $\mathbf{w}_{\mathbf{a}}$ it is possible to understand which original variables are summarized into the new latent variable $\mathbf{t}_{\mathbf{a}}$. X-variables with high weights are highly correlated with Y-variables. Similarly, the Y-weights, $\mathbf{c}_{\mathbf{a}}$, provide information how the Yvariables are summarized in the score vector $\mathbf{u}_{\mathbf{a}}$. 
PLS addresses the relationship "process" $\mathrm{X} \Rightarrow$ results $\mathrm{Y}$, by making a model of $\mathrm{X}$, and a connected model of $\mathrm{Y}$

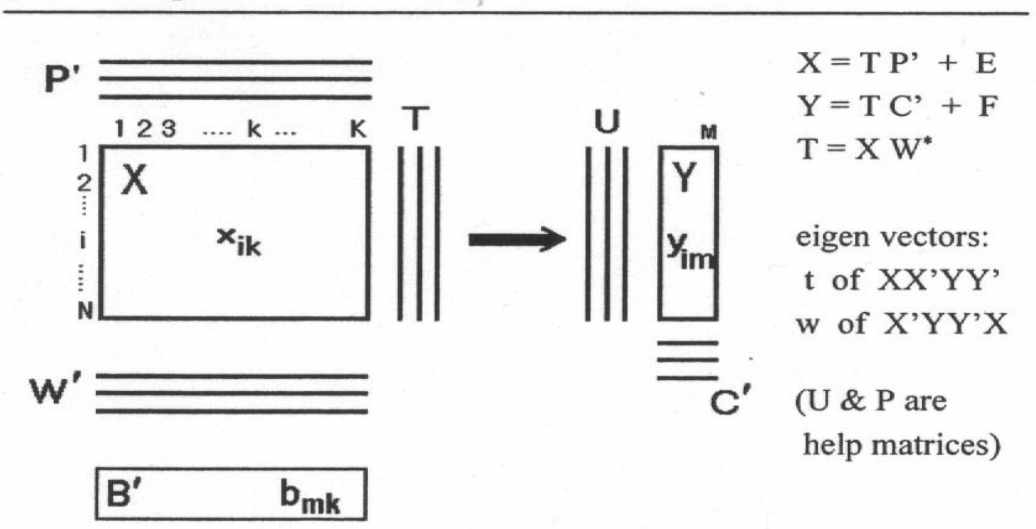

Figure 3.9 PLS Matrices (Umetrics, Inc. 2003)

In summary, PLS forms "new x-variables", $\mathbf{t}_{\mathbf{a}}$, as linear combinations of the old ones, and thereafter uses these new $\mathbf{t}$ 's as predictors of $\mathbf{Y}$. For each component (a), the parameters, $\mathbf{t}_{\mathbf{a}}, \mathbf{u}_{\mathbf{a}}, \mathbf{w}_{\mathbf{a}}, \mathbf{p}_{\mathbf{a}}$, and $\mathbf{c}_{\mathbf{a}}$ are determined by a PLS algorithm. The PLS model is expressed as a set of $\mathbf{X}$-score vectors, $\mathbf{Y}$-score vectors, $\mathbf{X}$-weight and $\mathbf{Y}$-weight vectors, and a set of PLS model dimensions. Each component or dimension (a) expresses a linear relation between an $\mathbf{X}$-score vector $\left(\mathbf{t}_{\mathbf{a}}\right)$ and $\mathbf{Y}$-score vector $\left(\mathbf{u}_{\mathbf{a}}\right)$. The weight vectors of each model dimension express how the $\mathbf{X}$-variables are combined to form $\mathbf{t}_{\mathbf{a}}$, and the $\mathbf{Y}$ variables are combined to form $\mathbf{u}_{\mathbf{a}}$. In this way the data are modeled as a set of "factors" in $\mathbf{X}$ and $\mathbf{Y}$ and their relationships. 


\subsubsection{Mathematical Interpretation of PLS - Classical PLS Algorithm}

Lindgren et al. (1992) state, "Partial least squares (PLS) regression is a latentvariable-based method for linear modeling of the relationship between a set of response variables $\mathbf{Y}($ size $\mathrm{N} \times \mathrm{M})$ and a set of predictor variables $\mathbf{X}(\operatorname{size} \mathrm{N} \times \mathrm{K})$. The objective was to find relations between blocks of data relating their latent variables.

“The 'classical' PLS regression algorithm is based on further developments of the NIPALS (non-linear iterative partial least square) method presented by Wold. NIPALS is a robust method of solving eigenvector-eigenvalue-related problems where the eigenvectors (components, factors) are calculated in a partial fashion, one at a time, until all the variance in the data structure is explained. For each new dimension the information explained by the last component is subtracted from the data matrices $\mathbf{X}$ and Y to create residuals, on which subsequent dimensions are calculated by the same procedure."

"The objective of using PLS is to understand the influence of $\mathrm{X}$ on $\mathrm{Y}$ and to develop a model for predictive purposes. The final result is a set of PLS weights (w and c) and loadings (p) which for predictive purposes may be converted into a set of PLS regression coefficients (B).” (Lindgren et al. 1992) The PLS solution is typically written as:

$$
\mathbf{Y}=\mathbf{X B}+\mathbf{F}
$$

where $\mathbf{Y}$ is response matrix $(\mathrm{Nx} M): \mathrm{N}=$ observations; $\mathrm{M}=$ response variables

$\mathbf{X}$ is the predictor matrix $(\mathrm{N} \times \mathrm{K}): \mathrm{N}=$ observations; $\mathrm{K}=$ predictor variables

$\mathbf{B}$ is the matrix of regression coefficients

$\mathbf{F}$ is the matrix of residuals 
The regression coefficient matrix, $\mathbf{B}$, is expressed as:

$$
\mathbf{B}=\mathbf{W}\left(\mathbf{P}^{\prime} \mathbf{W}\right)^{-1} \mathbf{C}^{\prime}
$$

where $\mathbf{W}$ is the matrix of $\mathbf{X}$-weights $(\mathrm{K} \times \mathrm{A})$ : $\mathrm{A}=$ rank index

$\mathbf{P}$ is the matrix of $\mathbf{X}$-loadings $(\mathrm{K} \times \mathrm{A})$

$\mathbf{C}$ is the matrix of $\mathbf{Y}$-weights (M x A)

The regression coefficient matrix, B, depends only on the loading and weight vectors for the PLS decomposition. The score vectors $\mathbf{t}$ and $\mathbf{u}$ are not needed to write a PLS solution.

The classical PLS algorithms approach as discussed by Lindgren et al. (1992) is as follows. For each dimension or component $(a=1,2, \ldots, A-1, A)$ the first 5 steps are iterated until convergence, meaning the vectors do not change by more than a small amount usually a rounding off error. The next three steps, 6 to 8 , are calculated after convergence.

The PLS algorithm is given here for just the first component.

$$
\begin{aligned}
& \text { Step } 1 \mathbf{w}^{\prime}=\mathbf{u}^{\prime} \mathbf{X} / \mathbf{u}^{\prime} \mathbf{u} \\
& \text { Step } 2 \mathbf{w}=\mathbf{w} /\|\mathbf{w}\| \quad(\mathbf{w} \text { is normalized to norm one }) \\
& \text { Step } 3 \mathbf{t}=\mathbf{X w} / \mathbf{w}^{\prime} \mathbf{w} \\
& \text { Step } 4 \mathbf{c}^{\prime}=\mathbf{t}^{\prime} \mathbf{Y} / \mathbf{t}^{\prime} \mathbf{t} \\
& \text { Step } 5 \mathbf{u}=\mathbf{Y} \mathbf{c} / \mathbf{c}^{\prime} \mathbf{c}
\end{aligned}
$$

The $\mathbf{u}$ in step 5 will go back to step 1 until convergence of the vectors. Here $\mathbf{w}$ is a $\mathrm{K} \times 1$ vector of PLS $\mathbf{X}$-weights, $\mathbf{c}$ is an M x 1 vector of PLS Y-weights, $\mathbf{t}$ is an $\mathrm{N} \times 1$ vector of PLS $\mathbf{X}$-scores and $\mathbf{u}$ is an $\mathrm{N}$ x 1 vector of $\mathbf{Y}$-scores. The next three steps are taken after convergence of steps 1 to 5 . 
Step $6 \mathbf{p}^{\prime}=\mathbf{t}^{\prime} \mathbf{X} / \mathbf{t}^{\prime} \mathbf{t}$

Step $7 \mathbf{E}=\mathbf{X}-\mathbf{t p}{ }^{\prime}$

Step $8 \mathbf{F}=\mathbf{Y}-\mathbf{t c}^{\prime}$

Where $\mathrm{p}$ is a $\mathrm{K} \times 1$ vector of PLS $\mathbf{X}$-loadings, $\mathbf{E}$ contains the residuals for $\mathbf{X}$, and $\mathbf{F}$ contains the residuals for $\mathbf{Y}$.

These steps are for the first component or dimension of the classical PLS algorithm. To determine addition components the residual matrices ( $\mathbf{E}$ and $\mathbf{F})$ are used as $\mathbf{X}$ and $\mathbf{Y}$ for the next dimension.

\subsubsection{PLS Model Interpretation}

\subsubsection{Outliers}

Plots of PLS scores are invaluable for an overview of the relationships among the observations, e.g. the relationship between $\mathbf{X}$ and $\mathbf{Y}$ on finding outliers in the $\mathrm{X}$ - or $\mathrm{Y}$ data. Observation diagnostics are used to determine if there is non-linearity and or outliers in the data. Outliers can be either strong or moderate. The strong outliers are found by inspecting the scores using Hotelling $\mathrm{T}^{2}$; while the moderate by looking at residuals using DModX. Eriksson et al. (2001) commented that "Observations outside the ellipse defined by Hotelling $\mathrm{T}^{2}$ deviate from normality; while observations exceeding the critical distance in DModX do not fit the model well."

\subsubsection{Scores}

Interpretation of the PLS model, begins with the scores, $\mathbf{t}$ and $\mathbf{u}$, that contain the information about the observations and about their similarities / dissimilarities with respect to the problem and model. When the scores are plotted against each other for the 
first few PLS dimensions, one obtains a view of the relationship between $\mathbf{X}$ and $\mathbf{Y}$ as well as relationships among the observations. Scores are weighted averages of the $\mathbf{X}$ and $\mathbf{Y}$ data and become "more precise the more numerical values are used as its basis; hence PLS works well with short and fat matrices - more columns than rows." (Eriksson et al. 2001)

PLS score plots are used to discover deviations from the $\mathbf{X} / \mathbf{Y}$ correlation structure. Plotting $\mathrm{t} / \mathrm{t}$ score plot is useful for uncovering deviation in the $\mathbf{X}$-data and a $\mathrm{u} / \mathrm{u}$ plot for looking at the $\mathbf{Y}$-data. Deviation in the X-data often corresponds to changes in the operating conditions in a process industry; while deviations in Y-data often indicate fluctuating or degrading quality in manufacturing processes.

The score plots can also be used to determine if there is departure from linearity between $\mathbf{X}$ and $\mathbf{Y}$. If there is departure then data transformation may be needed.

\subsubsection{Weights}

The weights, $\mathbf{w}^{*} \mathbf{c}$, on the other hand give information about how the variables combine to form the quantitative relationship between $\mathbf{X}$ and $\mathbf{Y}$. The weights are important in understanding which $\mathbf{X}$-variables are important (numerically large absolute $\mathbf{w}^{*}$ values), which $\mathbf{X}$-variables provide the same information (similar profiles of $\mathbf{w}_{\mathbf{a}}$ values), and for the interpretation of the scores, t. Plots of the scores and weights facilitate the model interpretation.

The PLS analysis results in model coefficients for the variables, called PLSweights. The weights for the $\mathbf{X}$-variables, denoted $\mathbf{w}$, indicate the importance of these variables, that is, how much they "in a relative sense" participate in the modeling of $\mathbf{Y}$. The weights for the $\mathbf{Y}$-variables, denoted by $\mathbf{c}$, indicate which $\mathbf{Y}$-variables are modeled in 
the respective PLS model dimensions. When these coefficients are plotted in a $\mathbf{w}^{*} \mathbf{c}$ plot, we obtain a picture showing the relationships between $\mathbf{X}$ and $\mathbf{Y}$, those $\mathbf{X}$-variables that are important, which $\mathbf{Y}$-variables are related to which $\mathbf{X}$, etc. It gives information about how the measured variables combine to form quantitative relation between $\mathbf{X}$ and $\mathbf{Y}$. The loading plots can also be used to interpret the patterns seen in the score plots. The loading and score plots are complementary and superimposable with the direction in one plot corresponding to the same direction in the other plot (Eriksson et al. 2001).

It is possible to plot the $\mathrm{X}$-weights $\left(\mathbf{w}^{*}\right)$ and, the $\mathrm{Y}$-weights (c) alone or plot both types of weights $\left(\mathbf{w}^{*} \mathbf{c}\right)$ in the same graph. It also helps to evaluate the structure modeled by looking at each component. The weights plot $\left(\mathbf{w}^{*} \mathbf{c}\right)$, provides an overview of the relationships between all factors and responses at the same time. It tells which variables are related / associated and which contribute unique information.

Weight plots also help interpret specifically how the predictor variables combine in regulating the response variable(s). This interpretation is accomplished by first drawing a line from the response variable through the origin on the weight plot. Then all predictor variables are projected orthogonally to the line. The position where each "projection line" intersects the line is used in the model interpretation. The predictor variables on the same side of the origin as the response variable are positively correlated to the response variable; while those on the opposite side of the origin are negatively correlated to the response variable. The further away a variable is from the origin the more influence or impact it has on the response variable. 


\subsubsection{Variable Influence on Projection (VIP)}

Another model interpretation tool is the variable influence on projection parameter, VIP. VIP is a squared function of the PLS weights, i.e. the sum of the squared, PLS weights, $\mathbf{w}^{*}$, taking into account the amount of explained Y-variance of each dimension. For a given model there is one and only one VIP-vector summarizing all components and Y-variables. Predictors with a large VIP, greater than 1, are most influential for the model. It is usually recommended to study VIP along with either weights or coefficients.

VIP can be used for a cautious variable selection process. A column plot of VIP typically looks like a formation of "stairs". And a plot of VIP might point to one or several natural thresholds that might for be used for discriminating between important and unimportant predictors. In most cases a cut-off around 0.7 to 0.8 is typically used.

\subsubsection{Residuals}

Reviewing the PLS residuals, $\mathbf{E}$ and $\mathbf{F}$, helps to identify moderate outliers. The detection tool for moderate outliers is called DModX/DModY that is the distance the observation is from the model in $\mathrm{X} / \mathrm{Y}$-space. DModX and DModY are based on row-wise summation of the elements in the residual matrices $\mathbf{E}$ and $\mathbf{F}$. The tolerance volume in $\mathbf{X}$ and $\mathbf{Y}$ is indicated by a solid line, D-Crit. The critical distance is computed for most applications for the 0.05 probability level, but may be changed. There is no D-Crit for DModY. 
The calculation of DModX and DModY are based on summation of the residual matrices. The residual observation variance, $\mathrm{S}^{2} \mathrm{OX}$ is computed as

$$
S^{2} O X=\sum_{k} e_{i k}^{2} / D F
$$

The residual observation variance is converted to absolute distance DModX as,

$$
\operatorname{DModX}_{a b s}=\sqrt{S^{2} O X}
$$

Or the normalized distance DModX as,

$$
\operatorname{DModX}_{n o r m}=\sqrt{S^{2} O X / \operatorname{variance}(E)}
$$

The formulas for calculating DModY are the same except for substituting residual $\mathrm{f}_{\mathrm{im}}$ for residual $\mathrm{e}_{\mathrm{ik}}$.

\subsubsection{Variable Diagnostics}

Variable diagnostics, $\mathrm{R}^{2} \mathrm{VX}$ and $\mathrm{R}^{2} \mathrm{VY}$, are used for determining which predictors are important and which responses are explained well. Again the residual matrices are summed however this time it is column-wise. If it is performed for the X-residuals in matrix $\mathbf{E}$ it is possible to compute the explained variation, $\mathrm{R}^{2} \mathrm{VX}$, for a variable. The $\mathrm{R}^{2} \mathrm{VX}$ ranges from 0 (no explanation) to 1 (complete explanation). $\mathrm{R}^{2} \mathrm{VX}$ is determined by first calculating SSVX the residual variable variation. $S S V X=\sum_{i} e_{i k}^{2}$. This is transformed to $\mathrm{S}^{2} \mathrm{VX}$, the residual variable variance, by dividing by the appropriate degrees of freedom. The cumulative explained variation after the $\mathrm{A}^{\text {th }}$ component of a variable is calculated as follows

$$
R^{2} V X(\text { cum })=1-S S V X[A] / \operatorname{SSVX}[0]
$$

where A represents the number of PLS components. 
The size of the Y-residuals shows which responses are well accounted for by the PLS model. This information is given by the explained variation, $\mathrm{R}^{2} \mathrm{VY}$ based on the $\mathrm{Y}-$ residuals in matrix $\mathbf{F}$. The computation of $\mathrm{R}^{2} \mathrm{VY}$ is computed as follows. First the residual response variable variation SSVY is computed as $S S V Y=\sum_{i} f_{i m}^{2}$ which when

divided by the appropriate degrees of freedom gives $S^{2} V Y . \quad S^{2} V Y=\sum_{i} f_{i m}^{2} / D F$. The cumulative explained variation is given by

$$
R^{2} V Y(\text { cum })=1-S S V Y[A] / S S V Y[0]
$$

where A represents the number of PLS components.

\subsubsection{Coefficients}

PLS regression coefficients may be obtained as part of the PLS solution. The relationship between the PLS regression coefficients and the PLS weights are given by:

$$
\mathbf{B}=\mathbf{W}\left(\mathbf{P}^{\prime} \mathbf{W}\right)^{-1}=\mathbf{W} * \mathbf{C}
$$

PLS coefficients aid in model interpretation particularly when there are several components ( $>4-5)$ in the PLS model. The big advantage is one vector of concise model information per response, not several vectors of weights. The size of the coefficients indicates the influence of each model term.

\subsubsection{Cross Validation}

The number of components needed by the PLS model is determined using a technique called cross-validation. The number of components is determined as the optimum balance of the fitting and the predicting ability of the model. Fit is how well mathematically the "training" data can be reproduced; while predicting ability is how 
reliable is the model to predict the outcome of future experiment. The goodness of fit is given by $\mathrm{R}^{2}$ (=explained variation); while goodness of prediction is given by $\mathrm{Q}^{2}$ (=predicted variation). In PLS the $\mathrm{R}^{2}$ and $\mathrm{Q}^{2}$ refer to the model behavior with regards to Y-data, the responses.

$\mathrm{R}^{2}$ is inflationary and will continue to increase to 1 (complete explanation) as the model becomes more complex or as more components are added. $\mathrm{Q}^{2}$ on the other hand is not inflationary and will not automatically approach 1 as the model becomes more complex or as components are added. The number components needed then is determined at the stage when the $R^{2}$ and $Q^{2}$ values depart, that is the $R^{2}$ value continues to increase while $\mathrm{Q}^{2}$ levels off or declines. There is no needed to add addition components when the predictive ability does not increase to any appreciable extent. By examining the $\mathrm{R}^{2}$ and $\mathrm{Q}^{2}$ for individual responses it is possible to determine which responses are well modeled and which are not.

The cross validation technique is performed by dividing the data into a number of groups, e.g. five to ten. Partial models are developed from the reduced data when one of the groups is omitted. As each partial model is developed the omitted data is used as a test data set. The differences between the actual and the predicted Y-values are calculated from these data points. The sum of squares of these differences are computed and collected to form PRESS (predictive residual sum of squares), which is a measure of the predictive ability of the model. PRESS $=\sum\left(y_{i k}-\hat{y}_{i k}\right)^{2}$.

When cross validation is conducted in a sequential manner, $\mathrm{PRESS}_{\mathrm{a}} / \mathrm{SS}_{\mathrm{a}-1}$ is evaluated after each component, and a component is judged significant if the ratio is smaller than 0.9 for at least one of the $\mathrm{y}$-variables. $\mathrm{SS}_{\mathrm{a}-1}$ is the (fitted) residual sum of 
squares before the current component (a). The calculations continue until a component is non-significant.

A PRESS is calculated for the final model with the estimated number of significant components. This is often re-expressed as $\mathrm{Q}^{2}$ (the cross validated $\mathrm{R}^{2}$ ), a statistic similar to $\mathrm{R}^{2}$.

$$
\begin{aligned}
& R^{2}(Y)=1-R S S / S S Y_{\text {tot.corr }} \\
& Q^{2}(Y)=1-P R E S S / S S Y_{\text {tot.corr }}
\end{aligned}
$$

where: SSY tot.corr is the total variation in the $\mathrm{Y}$-matrix after mean centering.

In evaluating $\mathrm{R}^{2} / \mathrm{Q}^{2}$ there are some guidelines by Eriksson et al. (1999). First, without a high $\mathrm{R}^{2}$ it is impossible to get a high $\mathrm{Q}^{2}$. Second, a $\mathrm{Q}^{2}>0.5$ is considered good, and a $\mathrm{Q}^{2}>0.9$ is excellent. Third, the difference between $\mathrm{R}^{2}$ and $\mathrm{Q}^{2}$ should not be greater than 0.2 to 0.3 .

\subsubsection{Prediction}

When the PLS model is considered reliable, i.e. after reviewing t/u plots, $\mathrm{w}^{*} \mathrm{c}$ plots, cross validation, coefficients, etc., the model may be used for predicting Y-data from future $\mathbf{X}$-data. A new observation is judged similar to the "training set" if its score is projected to the X-plane and falls within the Hotelling $\mathrm{T}^{2}$ ellipse. It can then be entered into the $t / u$ inner relationship, thus producing a $u$-value for that dimension. The $u$-value defines a location on the Y-plane that corresponds to the predicted value for the response variable. 
It is possible to calculate prediction intervals in PLS to demonstrate the certainties

of the predicted $\mathrm{Y}$-values. The variance of the predicted $\hat{Y}$ for a given response y at a point $\mathrm{X}_{0}$ is computed as:

$$
V(\hat{Y})=\left(\frac{1}{N}+X_{0}^{\prime}\left(T^{\prime} T\right)^{-1} X_{0}\right) \sigma^{2}
$$

$\sigma^{2}$ is the y error variance estimated from the sum of squares of the residuals divided by the degrees of freedom for PLS i.e. $\left(\mathrm{N}-\mathrm{A}-\mathrm{A}_{0}\right), \mathrm{N}=$ number of observations in the training set, $\mathrm{A}=$ number of PLS components and $\mathrm{A}_{0}$ is 1 or 0 depending on whether $\mathrm{Y}$ is centered or not. The confidence intervals can be calculated from the standard error (square root of the variance) by multiplying by a t value with the appropriate degrees of freedom.

\subsection{Multivariate Modeling}

Multivariate process modeling is used when multiple variables and multiple responses are of interest. In dryer modeling, the multivariate approach to investigate process data is desirable for several reasons. First, a drift in a process often occurs with groups of variables moving up or down together and the drift may not be detected by looking at one variable at a time. Eriksson et al. (1999) comments, "the information sits in the variable correlation pattern and not in the individual variables." Second, when samples show up that break the general correlation structure, the diagnostics / graphs can assist in spotting the change and then determining which variable(s) changed that caused the break. Third, the multivariate approach based on Projection to Latent Structures by Means of Partial Least Squares (PLS) has several useful diagnostic tools that aid in root cause analysis. Eriksson et al. (2001) stressed that PLS works well when modeling processes where "no good fundamental theory" exists. 
Multivariate modeling can be used to supervise existing processes, improve them, and even help in developing new processes. In monitoring the state of a process by multivariate modeling, early warning signals when the process shifts and even root cause information can be used to improve the process. Measuring process data and modeling the data increases the understanding of the process and the relationships between different parts of the process. Understanding how the output is affected by input and process variables helps with improving product quality and reducing product costs, but probably most importantly helps keep the process under control.

In modeling processes, it is important to trust only modeling results that are both interpretable and show good predictive ability. In addition, model parameters should be stable under validation, and one should be careful with model interpretation if the model does not predict well in validation. Likewise be skeptical with predictions from a model that is difficult to interpret logically.

PLS is used in process data analysis to model the relationships between blocks of variables such as process factors $(\mathrm{X})$ and quality measurements $(\mathrm{Y})$. Process modeling using PLS helps to find relationships between the process variables measured overtime and the response / resultant variables such as strength properties. Martens and Naes (1989) refer to this as "calibration" which is the "art of using empirical data and prior knowledge for determining how to predict unknown quantitative information $\mathrm{Y}$ from available measurements X via some mathematical transfer function."

The PLS score vectors are new variables from the model that summarize the many process variables into a few scores that can be monitored separately. In addition, 
"DModX and DModY summarize the variation of the process that is "un-modeled", and they can be used to uncover new process events" (Eriksson et al. 1999).

An objective in process data analysis is to establish which process factors are most influential and to develop a prediction model that can be used to improve the process and/or used on-line to monitor the process. Designed experimentation with the process is recommended to provide reliable information on the relation between input and process variables and output (response) variables. To fish for relationships between input and output in a process data historian is risky and often less successful. This is because a process does not provide data with good information content when the important factors are well controlled within small "control intervals". In designed experimentation the controllable process variables are explored using a DOE, design of experiment, and treatment combinations set up. Each treatment combination will be run for a time period to capture process variation and the response variables measured. The designed experimental results will contain $\mathrm{N}$ observations and $\mathrm{K} \mathrm{X}$-data and $\mathrm{M}$ Y-data that are recorded on some predetermined time basis. The process of developing the PLS model typically begins with a Principal Component Analysis (PCA) overview of all the data. Outliers are investigated using the score plots and the loading plots. Outliers represent "extreme process conditions" and are usually dropped so they do not overly influence any model developed to represent "normal process conditions". The model approximation is "better the greater the similarity between observations and the greater the number of model components" (Eriksson et al. 2001).

A new PCA is then conducted and cross validation is used to determine the number of relevant components. If the outliers are removed, the score plot should show a 
plot with clustering of the observations arising from the designed experiment, treatment combinations. The score plot shows the correlation between observations and helps to discern any groups or trends in the data. The loading plot shows the correlation between the variables and can be used to compare with the score plot. The comparison helps in understanding the relationship between variables and observations.

Prior to running the PLS, Eriksson et al. (1999) indicated expanding the X-matrix to include cross products and second order variables may be in order to improve the model. The PLS is then run and the model is reviewed. $\mathrm{R}^{2} \mathrm{Y}$ and $\mathrm{Q}^{2}$ values are examined to determine the amount of variation in the response variables the model explains and predicts.

In the evaluation of the PLS model, information about the relationship between the $\mathbf{X}$ and $\mathbf{Y}$ variables can be determined by looking at the $\mathrm{t}$ and $\mathrm{u}$ score plot for each dimension. The relationship should be linear and the correlation will become less as the dimension or component number increases. The DModX can also be evaluated to see if there are any moderate outliers.

The interpretation of the model is typically conducted by studying the $t_{1} / t_{2}$ score plot and the $\mathrm{w}^{*} \mathrm{c}_{1} / \mathrm{w}^{*} \mathrm{c}_{2}$ weight plot. The weight plot may allow assigning meaningful attributes to the components based on the clustering or grouping of the variables. The weight plots may allow the discrimination between observations both in the first and second components. The variables on the outer fringes of the plots have the greatest influence in the model; while those toward the center of the plot have low modeling influence. 
Another way to review the importance of the different X-variables is to study the variable influence on projection (VIP) plot. VIP is very important when the model has many components and covers many responses. To determine the relevance of the Xvariables identified as most important in the VIP plot for the different responses, it is useful to look at response contour plotting. It is important to note, however, that a proper response contour plot can only be created for a model where all factors have been manipulated according to design of experiments (DOE). The response contour will plot the important X-variables on the axes and the response variable results in the field.

The full PLS model may be used for process monitoring. The reasons the full model is used are several. "The on-line measurements enable on-line prediction, and by using all variables the correlation structure among all variables will aid in stabilizing the predictions. In addition, for early fault detection, it is advantageous to employ many descriptors rather than a few, as this generally implies that more types of process upsets and problems can be discovered" (Eriksson et al. 1999). Hotelling's $T^{2}$ plot, $t_{1} / t_{2}$ plot, and DModX, all help to discover extreme situations.

The full PLS model can also be used for on-line predictions. Eriksson et al. (2001) uses a time series plot of the predicted levels of response variables and also plots the DModX directly below. Where the DModX goes above the D-Crit line indicates regions in time where the on-line predictions are less reliable.

\subsection{Multivariate Statistical Process Control (MSPC)}

MSPC is a method of combining multiple variables into a single chart. Univariate control charts imply that the process and quality variables are independent of each other and should be monitored alone. In actuality, because many variables are measured on a 
process, many are partially correlated if not strongly correlated and therefore should be monitored together. Information is often found in the correlation pattern between variables and not just the individual variable by itself. Because univariate control charts ignore the correlations, they are often inadequate in the continuous process industries of today.

One of the more popular multivariate control charts is based on Hotelling $\mathrm{T}^{2}$ statistic. Hotelling $\mathrm{T}^{2}$ statistic is a measure of statistical distance and is a weighted summary of all scores. It gives an estimate of how far away an observation is from the center of a PLS or PC model hyperplane.

For a $p$-dimensional observation vector $X^{\prime}=\left(x_{1}, x_{2}, \ldots, x_{p}\right)$, the $\mathrm{T}^{2}$ statistic is given as $\mathrm{T}^{2}=(X-\bar{X})^{\prime} S^{-1}(X-\bar{X})$,

where the mean vector $\bar{X}$ represents the process center or the average value of observations on many process variables. The covariance matrix, S, provides information on the relationships between the variables of the observation vector. The $T^{2}$ value of an observation vector "measures how far the observation is from the process center relative to the covariance matrix $\mathrm{S}$ or relative to the scatter of points used to compute $\mathrm{S}$ " (Mason and Young 2000).

Eriksson et al. (2001) presents the Multivariate Shewhart chart, the Cusum chart and EWMA chart where the PLS-scores $t$, can be plotted on each type chart. The scores $t_{i}$, for each component can be plotted to see how the process evolves over time with respect to the model dimension or component. Scores are less noisy than the original variables as they are weighted averages. 
A more popular alternative is to plot the Hotelling $\mathrm{T}^{2}$ statistic. It is a "weighted summary of all the scores and gives an estimate of how far away an observation is from the center of a PLS-model hyper-plane" (Eriksson et al. 2001). In addition, by plotting it on a Shewhart chart, one is able to "detect strong deviations in the systematic part of the data" (Eriksson et al. 2001).

There are several problems with using the Hotelling $\mathrm{T}^{2}$ statistic. First it is a "squared function of the PC-scores and only gives the absolute magnitude of a process deviation and the sense of direction in which the process is moving is lost" (Eriksson et al. 2001). A second "problem that arises in using a multivariate $T^{2}$ statistic is the interpretation of a signal. This arises as a result of attempting to reduce a $p$-dimensional data vector into a uni-dimensional statistic" Mason et al. (1997). With MSPC, an out of control signal can arise for two basic reasons. First reason might be that one of the $p$ variables is out of control. Second reason might be the correlation structure between two or more of the variables may have changed. The problem is determining which variable(s) is out of control.

Jackson (1991) discusses decomposing the $\mathrm{T}^{2}$ statistic into a sum of $p$ components and using these components to solve the identification issue. Mason et al. (1995) believe there are draw backs with this method as it is difficult to attach meaning to the various components much less determine which variable is out of control.

Minitab ${ }^{\circledR}$ has a built in routine with its multivariate $\mathrm{T}^{2}$ control chart program that makes it easier to determine whether a variable has shifted or the correlation structure has changed. When an observation is out of control, the variable(s) that is most likely out is 
listed along with a p-value. Plus, the program has a "generalized variance" control chart that helps to monitor the correlation structure of the process.

Mason et al. (1995) proposed another approach to solving the identification issue that involves decomposition of the $\mathrm{T}^{2}$ statistic into individual components, each of which reflects the contribution of an individual variable. "The complete decomposition of the $\mathrm{T}^{2}$ statistic into $p$ independent components is possible. For example, three variables produce six different decompositions, while four variables would yield 24 decompositions." Then each component of the decomposition can be compared to a critical value to signal if the component is significant. 


\section{METHODS AND MATERIALS}

"The aim is admirable, but the method is madness." Republican minority report of the Joint Economic Committee 1977

\subsection{OSB Operations - OSB Process Flow and OSB Mill Modeling}

A typical OSB manufacturing operation consists of seven processing steps with each having multiple sub-steps. The first process step is log preparation that includes log storage, log conditioning, and debarking. The next step is stranding, followed by strand drying that in most cases includes dry strand screening. Strand blending follows the drying step and includes the application of various additives such as resin and wax. Then, the mat is formed as step five, followed by mat pressing. Finally, the panel is finished as step seven and includes cut to size, sanding, and profiling followed by edge sealing and packaging.

The OSB operation, that both the OSB Process Flow model and the OSB Mill model were to represent, has several variances from the steps described above. The differences include after stranding, a sub-step of green screening whereby the fraction of small furnish particles are removed before drying. This sub-step improves drying efficiency by not having to dry furnish that will later be screened out. The drying processing step is different in that it has two dryers in series; while, most OSB operations have only one dryer. By having the dryers in series, the residence time is increased, allowing the dryers to operate at lower temperatures. The blending step has added flexibility with the ability to use different resins including liquid and powder phenol formaldehyde and polymeric dimethyl diphenol diisocyanate (pMDI) depending on the type of structural panel the operation is manufacturing. Another variance is in the forming step with a larger mat size, 12 -ft by $24-\mathrm{ft}$; where most OSB mats are 8 -ft by 24 - 
$\mathrm{ft}$. Another is recycled material from the side trim saws and the flying cut off saws going back into the core furnish after blending; while rough trim from finishing goes back into the core dry bins to be blended again.

While the mill model was designed to depict or represent this particular OSB operation with its variations to the typical OSB operation, the OSB Mill model has numerous variables that can be changed to accommodate the differences between mills such as mat size, resin type, recycled material, etc.

\subsection{Dryer Heat Source and Rotary Dryer - Dryer Modeling}

The typical OSB dryer system consists of a heat source providing energy to a dryer drum or multiple dryer drums. In this particular case, the dryer system consists of a heat source providing heat to two sets of dryers with both sets having triple-pass drums in series. This unique design, having drums in series, allows the dryer system to operate at a lower inlet temperature thus minimizing volatile organic compounds (VOC) emissions. Specifics about the equipment modeled are listed in Table 4.1. 
Table 4.1 Drying Equipment Information

\begin{tabular}{lll}
\hline EQUIPMENT & MANUFACTURER & DESIGN DATA \\
\hline Heat Source & GTS Energy, Inc. & Provides heat energy for: dryer, press, \\
& & log thaw and buildings. \\
& Rating: $175 \mathrm{MMBTU} / \mathrm{Hr}$ \\
& Grate Area: $516 \mathrm{sq} \mathrm{ft}$ \\
& Fuel: bark and fines from log process \\
& and sander dust and saw dust \\
& & Type: Rotary triple pass \\
Dryers & Feed rate: 65,000 OD lbs $/ \mathrm{hr}$ \\
& Destec Amercia Inc. & from $100 \%$ to $5 \%$ \\
& Gas flow is concurrent \\
& Diameter $13 \mathrm{ft}$ \\
& Length each drum $62 \mathrm{ft} /$ total $130 \mathrm{ft}$ \\
& Length to diameter ratio of 9.4 \\
& Rotational speed $6 \mathrm{rpm}$ \\
& Holdup is $15 \%$ with a Residence time \\
& of approximately 10 minutes \\
\hline \hline
\end{tabular}

\subsubsection{Heat Source - GTS Energy, Inc.}

The heat source for the dryer is a wood fired burner system manufactured by GTS Energy, Inc. Figure 4.1 is a human machine interface (HMI) computer screen picture that shows the various parts of the heat source.

The wood residue used in the heat source consists of bark and fines from the log processing function as well as sander dust and sawdust from the finishing processing function. The mixing of the four fuel sources is important as too much dry fuel, sawdust and sander dust, can create "sparklers" and possible dryer fires; while too much wet fuel, bark and fines, reduces the efficiency of the system.

The heat source provides heat for both the dryers and the log thaw, press, and building heat, hot oil system. The hot oil system used for pressing and log thaw is the primary control loop in the heat source control system with the dryer system the secondary control loop. This simply means that if the hot oil system should demand more energy than the heat source can provide at that particular time, the heat source will cut 
back on the heat energy it provides the dryers until the hot oil system has reached its set point.

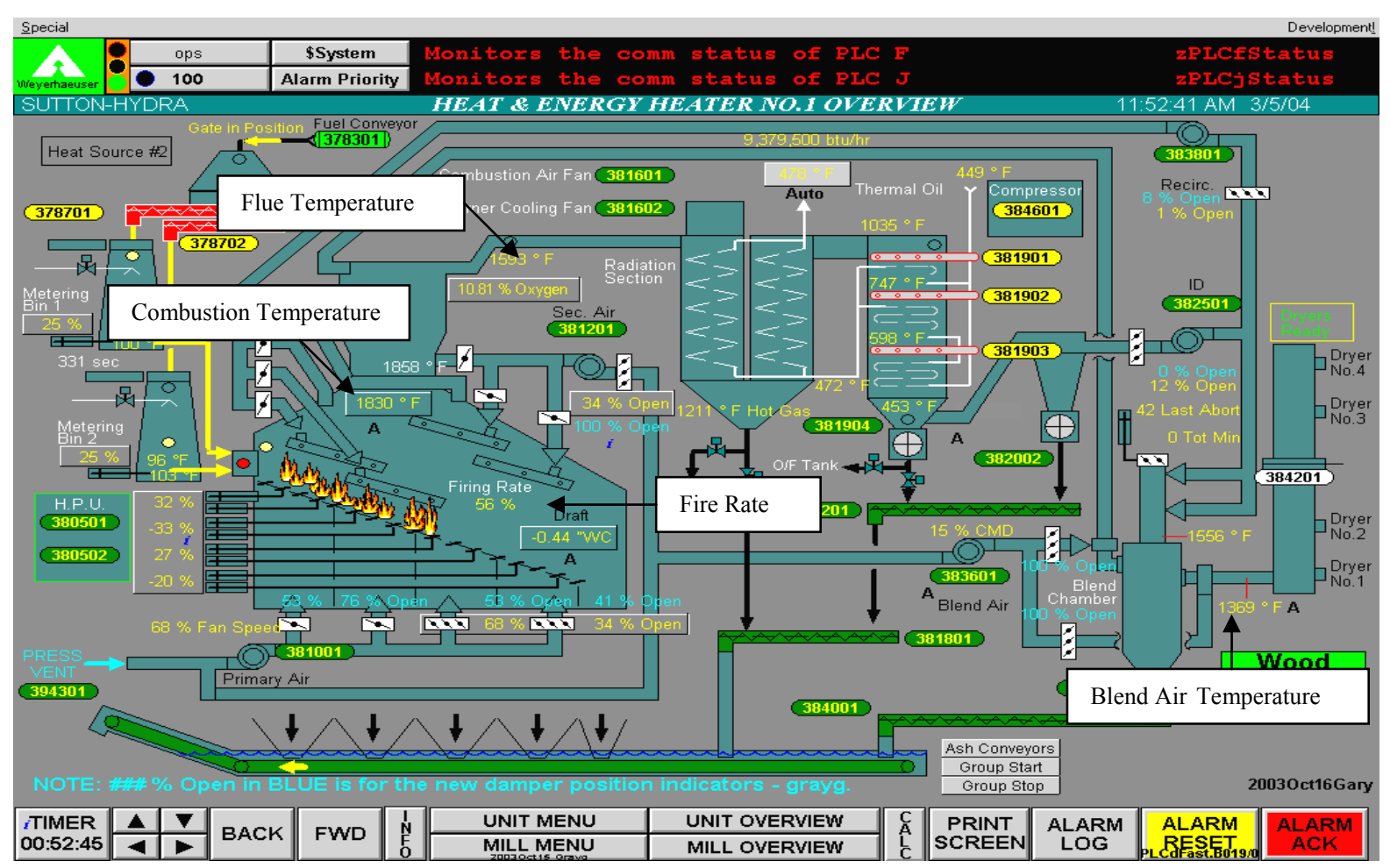

Figure 4.1 WonderWare Computer Screen of the Heat Source (GTS Energy, Inc.)

Furnish to the dryers comes from a wet, "surge" bin used to smooth out the flow from the strander operation. The flow of furnish from the bin is conveyed by screw conveyor to a weigh belt that feeds a chute to the dryer infeed. Mounted over the weigh belt is a moisture detector that measures inlet moisture content. The furnish is conveyed through the triple pass drums by the lifting action of the flights and air flow created by the dryer induced draft (ID) fan on the outlet. This is a unique dryer system with two drums in series. The material is transported through the first drum by literally "flying" down the first pass, it then reverses its direction and heads down the second pass; finally it reverses its direction again and goes down the third pass. From here a short duct section 
transports it to the second drum, and the material flows through the second drum like it did the first drum. After the second drum, the furnish flow is separated into two streams to go into high efficiency cyclones where furnish is separated from the air stream. The furnish drops down a chute into a flight conveyor where it is conveyed to dry bins located in the blending area. As the material is falling down the chute, part of it falls on to a "sampling" conveyor that conveys material under a moisture detector/meter measuring outlet moisture content of the dried strands.

Located at the inlet to the dryer ID fan is a pressure sensor that is monitored by the operator. This sensor provides data that indicates when too much material is being fed into the dryer. When the static pressure falls below -11" W.C., the system indicates furnish may be "plugging" somewhere in the system - cyclones, dryer, duct work. The ideal static pressure is greater than -10.5" W.C. as it allows some latitude if the feed system were to "slug" or rapidly overfeed the process due to poor bin filling, extremely wet or high moisture content furnish, etc.

The heat source and dryer system operate on energy demand. The dryer outlet temperature and furnish load determine the energy required from the heat source. The heat source control system responds by increasing the firing rate and combustion gas temperature going to the blend air chamber. The blend air chamber mixes the combustion gases from the heat source fire chamber with press vent gases to provide the necessary temperature based on the set point input to the system and the demand from the dryer.

The heat source system begins with the ram, fuel feeder and ends with the blend air duct going to the dryers. The amount of fuel fed to the heat source is determined by the ram feeder setting that is usually set at 0.45 draw rate and the firing rate of the 
furnace. The draw rate means it is only stroking $45 \%$ of the maximum amount. The mill has determined for their fuel type and energy demand that a draw rate of 0.45 is ideal. The fuel feeder "pushes" fuel in at a rate higher than the actual firing rate to ensure the furnace is not starved for fuel.

The heat source grate system moves the fuel through four stages of burn. The first section is used to dry the wet fuel and is typically at a setting of 0.4 . The setting is a ratio of the maximum stroke count per minute that is typically one to two strokes per minute. The second section is used to "stir up" the fuel and is set at 0.48 , the fastest speed of the four sections. The third section is the complete burn section and has a setting of 0.38 . The last grate section is the slowest of the sections with a setting of 0.28 to retain the ash bed on the grate and minimize spark carry over. The objective is to keep the "flame front" location about three-quarters of the way down the grate sections. The actual grate speed settings are a function of the fuel moisture. Fuel with high moisture content requires more residence time and lower grate speeds.

The next part of the heat source operating system is the fan system, which consists of primary (under fire or grate) air, secondary (over fire) air, and flue gas recirculation air. The primary air is the air coming from under the grate sections. The furnace pressure loop controller modulates the motor speed of the primary air fan and controls the primary air. Four dampers that are manually adjusted to provide the needed air to each of the four grate sections control the distribution of the primary air. The adjustments are based on the fire line position on the grate sections and how much ash is retained on the fourth section. 
The secondary air is the over fire air and enters the combustion chamber above the grates. It is used to agitate or create turbulence in the furnace. It is designed to enhance the incineration occurring on the furnace grate and to complete the combustion of the gases. It also designed to "knock down" sparklers and control particulate carry over in the air stream. A multiplier controls the secondary air dampers in the control system that follows the control signal from the primary air controller. As the firing rate and primary air fan increase, the dampers open accordingly. It should be noted that as the moisture content of the fuel increases, less secondary air is required. Also there is less secondary air needed if higher combustion temperatures are needed.

The flue gas recirculation air is also an over fire air source, but it enters the combustion chamber from the rear and has little oxygen content as it is truly re-circulated air. Its purpose is to reduce nitrous oxide emissions by reducing the combustion temperature in the furnace to around $1600^{\circ} \mathrm{F}$. It cools the combustion gas temperatures without combustion since it is void of oxygen.

The combustion chamber is maintained in a negative pressure atmosphere. The pressure set point for the combustion chamber is adjustable based on the demand for more or less energy. If the pressure is lowered making the chamber more positive, the primary air fan speed will increase which will increase the fuel burn and the combustion temperature will increase. The flow of air from the primary air fan determines the firing rate. The combustion rate is higher when more air is introduced through the grates. If on the other hand the pressure setting is increased to make the chamber more negative, the primary air fan decreases, the burn slows back, and the combustion temperature will likewise decrease. 
The combustion gases flow through two passageways. One passageway goes to the hot oil system and then to the blend air chamber, while the other passageway goes directly to the blend air chamber. The blend air then goes to the dryers. Induced draft fans control both of the gas streams. The heat source induced draft (ID) fan controls the hot oil system. If more energy is needed, the heat source ID fan damper will adjust to increase its pull of combustion gases through the hot oil heat exchange section. The dryer ID fan controls the blend air chamber gas stream. The temperature set point on the blend air chamber determines how much high temperature combustion gas and how much ambient air is required to balance and match the set point temperature. The chamber also separates particulate from the combustion gas before it flows through the dryer drums.

The heat source system has two main control loops. The loops are the temperature control and the pressure control. The temperature control is a proportional integral derivative (PID) loop that controls the variable frequency primary fan with a separate output to the actuator on the secondary air fan and a third output to the fuel feeder and grate speeds. The pressure control is the furnace pressure PID loop that controls the furnace draft by modulating the primary air fan.

\subsubsection{Dryer System - Operation Using Outlet Temperature Control}

The dryer system consists of two dryer drums in series. Figure 4.2 shows the human machine interface (HMI) computer screen of the dryer system including wet and dry bins and the drums. It also shows the outlet temperature, inlet temperature, the hot air damper, and the outlet moisture meter. 


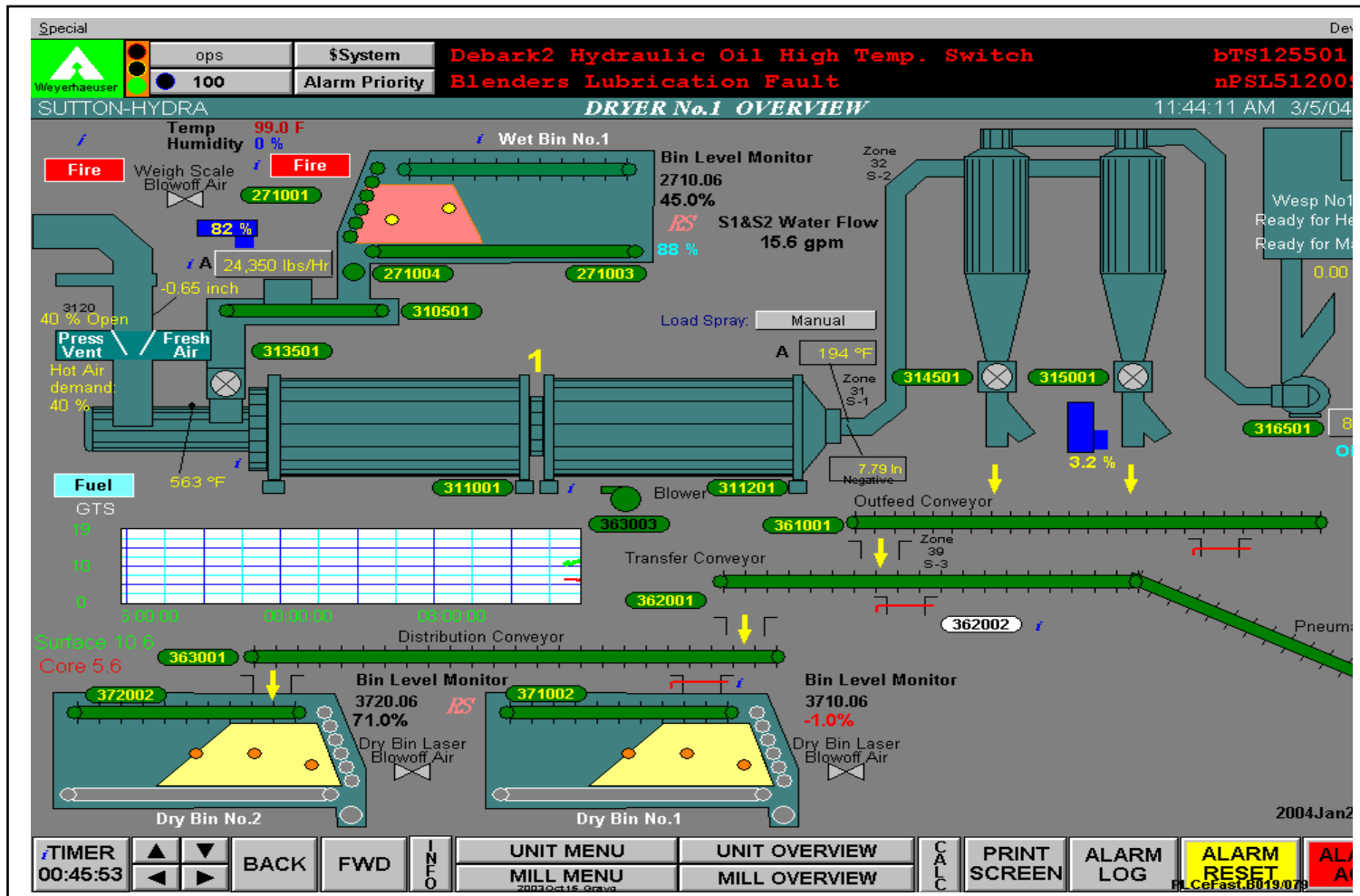

Figure 4.2 WonderWare Computer Screen of Drying System

The typical dryer control strategy is referred to as "outlet temperature control".

This means the hot air demand by the dryer is determined by the outlet temperature set point. If the set point is not being met, then the hot air demand will increase. The hot air demand is also referred to as the "heart valve opening". The heart valve dampers adjust to allow more / less blend air in comparison to less /more press vent and ambient air into the dryer. As the hot air demand increases, the blend air temperature may decrease which in turn will cause the heart valve to close minimizing the ambient air and the press vent air. If the blend air temperature cannot meet the demand, due to the heat source combustion temperature not being great enough, the firing rate will increase. This will cause the primary air dampers to open to increase the combustion temperature and the secondary air dampers will also open in proportion to the increase in primary air dampers. 
The dryer control is a temperature controlled PID loop. The loop will control the dryer temperature control damper actuator (as referred to as the heart valve). The process input is the dryer outlet temperature. The outlet temperature will be compared to the set point and the control loop will adjust its output signal to keep the process value at the set point value. Adjustments to the temperature set point will be made based on the furnish moisture content to be dried, the volume of furnish to be dried, the desired moisture content of the dried furnish, and climatic conditions, i.e. ambient humidity and temperature.

Furnish feed rate is controlled by a PID loop. The loop controls the wet bin bottom belt speed to discharge the set point weight or mass of material onto the nuclear scale belt. If the mass is not at set point, the controller will either speed up or slow down the belt speed until the set point is achieved.

A process control program change was made prior to the data collection phase that automated the feed rate change step. This change was implemented after an early trial indicated the "typical" feed rate changes caused the dryer process to become very unstable as observed by the outlet temperatures oscillating out of control. The automated feed rate change was designed to smooth out the process with the feed rate change increase set at about 330 pounds every minute.

\subsubsection{Variables for Dryer Modeling}

An "ideal" dryer control model would be based on the inlet temperature as the control variable as effected by 1 ) the incoming water load (moisture content of the wood furnish) and 2) the ability of the heat source to provide the required energy to evaporate the incoming water. The control program should hold the water load constant by varying 
the material flow, when the material moisture content changes. This would allow the inlet temperature to be held constant and would only change if the species mix or the furnish moisture content changed.

While the "ideal" dryer model could be based on the incoming water load, the current moisture measuring technology is limited to devices / instruments that on a real time basis can measure green moisture content to only within 5\% moisture content, one standard deviation, on an oven dry basis. This is not tight enough to be able to affect good control using inlet temperature.

Most commercial drying systems use outlet temperature as the control variable due to the difficulty in controlling the inlet temperature. The ability to maintain "tight" bounds on the inlet temperature was difficult, as determined by the author, during some earlier experimentation on the dryer.

\subsubsection{Retention Time Variable}

The water load is influenced by furnish retention time or drum holdup. The longer the furnish stays in the dryer with all other variables held constant, the lower the outlet moisture. The typical drum holdup is $15 \%$ of the drum volume as reported by Mujumdar (1989). The mill's dryer volume would be $\pi^{*} \mathrm{R}^{2} * \mathrm{~L}$ or 65,000 cubic feet with a holdup equal to $15 \%$, the volume of material would be 9,750 cubic feet. For the furnish processed at the OSB mill, the bulk density for four inch strands was determined to be approximately eight pounds per cubic foot, so the weight of material would equal 78,000 pounds. The retention time should increase as furnish mass increases. This increase in time should be discernible by the cross correlation statistic, i.e. the lag should decrease as the mass increases. 
The retention time is influenced by gas velocity. The dryer induced draft (ID) fan is used to "pull" furnish and combustion gases through the dryer with the ID damper used to control the "pull". Static pressure as measured on the outlet of the dyer is a method commonly used to determine the "speed" at which the strands are flowing through the dryers.

The design of the dryer flights and the state of repair of the flights can also affect the retention time. The purpose of the flights is to cascade the material through the hot gas stream and help move it along the length of the dryer. In addition the dryer rotation speed impacts retention time. Higher revolutions per minute increases the times the material will cascade and the quicker the material will move through the dryer thus reducing retention time.

\subsubsection{Heat Source / Inlet Temperature Variable}

The heat source has a blend air chamber where hot gases from the wood fired furnace are mixed with ambient air to control the blend air temperature. The control is accomplished by adjusting the ambient air flow and the air flow; allowing more or less ambient air to mix with the hotter furnace gas. In this particular heat source, the furnace temperature is affected by both the hot oil temperature demand that has priority over the dryers as well as the dryer demand.

\subsubsection{Study Variables for the Dryer Model}

Variables to Study / Manipulate in a designed experiment are:

1. Feed Rate or Gross Material flow - feed rate based on furnish weight.

2. Outlet Temperature 
Water load as measured by green furnish moisture content and blend air / inlet temperature had to be dropped as controlled process variables and became uncontrolled variables due to technology limitations of the green wood moisture meter and the inability to control the inlet temperature with the current control system.

\subsubsection{Instrumentation and Devices}

Numerous instruments and devices are used in the drying process to control the heat source, furnish weight, inlet and outlet temperatures, and fan dampers. Besides controlling the process, the instruments and devices can provide data that are collected during the operating shifts at scan rates as fast as every second and stored in the data historian.

The instruments and devices found with a typical OSB rotary dryer include the following: 1) moisture meters on both the inlet and outlet of the dryer provide information on the amount of moisture or water load entering the dryer and the amount of moisture leaving the dryer, 2) the nuclear weight scale on the infeed to the dryer providing data on the weight of the furnish entering the dryer, 3) the inlet and outlet resistance temperature devices (RTD's) giving data on the temperature entering and leaving the dryer, 4) the outlet static pressure gauge provides information about the condition of flow and whether plugging is imminent, and 5) the induced draft fan motor amps indicate the flow of furnish through the dryer.

The instruments and devices found on the heat source include the following. The damper actuators on the primary air, secondary air, and re-circulation air adjust to aid with the combustion of the fuel and with the distribution of oxygen in the furnace. The RTD's for combustion gas temperature, flue gas temperature, and blend air chamber 
temperature indicate temperatures. The actuators on the dampers for the forced draft fan and the induced draft fan aid in the distribution of air in the heat source.

There are also lasers used to measure the amount of furnish in the wet and in the dry surge bins before and after the dryer.

\subsubsection{Moisture Meters, Inlet and Outlet}

The inlet moisture meter is mounted over the dryer infeed weigh belt. Furnish from the wet bin is conveyed to the weigh belt by a screw conveyor that presents furnish to the weigh belt without "excessive" surging or clumping. The pile height is relatively even and the top of the pile is about ten inches from the moisture meter.

The outlet meter is mounted over a "sampling" conveyor mounted in the down chute for the discharge cyclone. The sampling conveyor is the result of this research project and collaboration with another OSB mill in Alberta, Canada. A portion of the furnish falls on the six-inch wide conveyor than conveys the material under the meter providing better "presentation" of the material to the meter.

The moisture meters manufactured by both Process Sensors and Moisture Systems use near infrared technology to measure the furnish moisture content. The strands are conveyed under or in front of the unit. A signal is sent to the material, and a reflected signal is received by the unit. Then using a calibration algorithm the unit displays the moisture content.

Passing individual samples of different known moisture contents in front of the sensor was used to develop the calibration algorithm. The meters initial readings were recorded as each sample was presented to the sensor; then the known moisture contents and the corresponding initial readings were keyed into the meter. The meter has a "built 
in" regression equation generator that regresses the initial readings against the known moisture content. This process was repeated several times until the equation has an $\mathrm{R}^{2}$ value of 0.90 or better.

The ability to determine moisture content of green strands is limited to $5 \%$ moisture content (OD), one standard deviation. On the other hand dry strands can be determined to within $0.5 \%$.

\subsubsection{Nuclear Scales}

The mass scale is manufactured by Bertholdt and uses a nuclear source to determine the mass of material feeding into the dryer. The scale is calibrated by comparing the mass balance for the entire process to the amount of material being processed by the dryer. Adjustments were made to the scale controller until the mass going under the source equals the mass balance for the process on a totalized basis for an hour.

\subsubsection{Data}

The data for building the model were collected from the process by using programmable logic controllers (PLC) and storing the data in a data historian. The data were recovered by a Microsoft Excel ${ }^{\circledR}$-program from the data historian. The data were then imported into SIMCA-P for data analysis and PLS model development.

\subsubsection{Tag Development and Aspen Tech IP21}

The instruments and devices provide data to the Allen Bradley Programmable Logic Controllers (PLC) that in turn sends the data to the data historian, IP2 $1^{\text {TM }}$ by Aspen Tech, for storage. Each instrument and device has a unique tag name that identifies it to 
the PLC and to the data historian. The data for the dryer model were collected at a scan rate of six seconds with the smoothing routine turned off. The process control profession refers to this smoothing routine as a "boxcar routine", and it is used to minimize the total amount of data that has to be stored. It only records data that "steps" outside the boxcar bounds. Consequently, the boxcar routine had to be turned off to so time series data could be collected

\subsubsection{Data Capturing Program}

Data used for the model was exported from the Aspen Tech data historian using an Excel® Add-in program developed by Aspen Tech to help with data exporting and data management. The data were averages of one minute duration.

\subsubsection{Design of Experiment - Dryer Modeling}

Controlled process variables to model the OSB dryer were initially inlet temperature, inlet moisture content, outlet temperature, and furnish feed rates. The design of experiment was to be a four-factor factorial design, $2^{4}$.

It was believed inlet temperature would provide quicker responses and system adjustments that in turn would reduce the large swings in moisture contents seen in the process. However during one of the pre-trials, it was determined that controlling with inlet temperature was not possible without considerable cost to re-program the control program. The outlet temperature on the other hand was easier to control as it was already part of the mill's dryer control program. Inlet temperature then became an intermediate results variable. 
Using inlet moisture content, it was believed the water load to the dryer could be determined, that could help with feed forward type control program. The variable had to be dropped when the technology, a near infrared (NIR) type meter, could not be reliably calibrated.

The data gathering technology for the response variable, outlet moisture content, was a true breakthrough for the study. The method that had been used for several years was a NIR moisture meter mounted on the down chute from the dryer out-feed cyclone, and dried furnish slid across the meter sensor. Several efforts to direct more or less furnish across the sensor failed to provide a consistent presentation of furnish to the meter, and the meter calibration was less than adequate. Another dryer data gathering approach was discovered by a colleague in Alberta, Canada in which a small conveyor about six inches wide and forty-eight inches long was inserted partially into the down chute so that a consistent sample of furnish falling down the chute could be extracted. The NIR meter was then mounted over the conveyor so furnish was consistently presented to the meter. This set up made calibration easy and data reliable.

The design of experiment for modeling the drying process was thus reduced to a two-factor factorial design with the process control variables or factors of interest being furnish feed rate and outlet temperature and their effect on the outlet moisture content. The levels studied were similar to the ones typically used during normal operations. Feed rate levels were 39,000 and 42,000 pounds per hour; and outlet temperature levels were 176 and 181 degrees F. The design was replicated twice with each run lasting from 30 to 60 minutes depending how the process was running. 
Other process variables studied to determine what amount of influence they might have on the outlet moisture content included 11 heat source variables, for example, combustion, flue gas, and blend air temperatures; while 16 dryer process variables included for example, green bin levels, bin operating speeds, inlet temperature, and ID fan amps. All together data from 29 variables were collected over the course of the trial.

\subsubsection{Data Collection}

Data were automatically collected or scanned during each run from the various devices, instruments, and PLC's and stored in the mill data historian, Aspen Tech's IP21. Process Explorer, a software package by Aspen Tech, charted the process variables during each run using time series graphs for 6 of the 29 variables.

As each of the 8 runs was completed, the data collection phase was temporarily suspended, and the next set of factor levels entered into the process control system. The data collection was resumed once the new levels were reached. In some cases as much as an hour was needed for the system to settle down and reach the next levels before data collection could be resumed.

During the trial, the data historian collected data at a scan rate of six seconds for the 29 variables. After the trial, the data from the historian were extracted using a program written using an Excel® add-in by Aspen Tech. The data were extracted as one minute averages, 9,135 data points - a data matrix of 315 observations by 29 variables. The data were then imported from the Excel ${ }^{\circledR}$ spreadsheet to SIMCA-P for further study. 


\subsubsection{Dryer Model Development}

This section covers the general principles used for dryer model development; while chapter 6 Dryer Model covers in more depth the actual process used to develop the Dryer Model.

Multivariate Data Analysis is frequently used in model development to represent manufacturing processes. The approach is to pre-process and evaluate the raw data, then to derive the model and interpret it, and finally validate the model. But it is not as simple as the three steps just listed. The analysis is very much an iterative procedure, a back and forth routine. It is quite normal to work through several cycles, using different ways to pre-process the data and involving different multivariate models, just to see how things change and how things are related.

Data evaluation focuses on understanding the regularities and peculiarities of the data. In most cases the raw data can rarely be used as they are in a database. The data may need to be stripped of outliers, and skewed data distributions may need to be transformed. In addition the data should be centered and scaled to remove variable dominance.

PCA can be used for a data overview, e.g. to look for outliers, to investigate groups and trends in the observations, and for evaluating the relationships among variables and between observations and variables. PLS is used to link the predictor variables $(\mathrm{X})$ with the responses $(\mathrm{Y})$ with the aim of being able to predict the responses from the future predictors. Then the diagnostic tools - scores, loadings, weights, DModX, cross-validation - are used to arrive at an optimal model. However, it may be found that it 
is necessary to go back a step to the data evaluation after running PCA/PLS and split the observations into groups, transform a variable(s) before arriving at the ultimate model. Before the PLS model may be used to predict or forecast future outcomes, the model has to be validated and confirm its predictive power. The model's forecasting ability may be tested by computing predictions for an external data or test set of observations. The model may also be evaluated to determine where to undertake further experiments, by inspecting contour plots of the response. The outcome may indicate an optimal model, or it may indicate model refinement such as eliminating variables is needed, and therefore it is necessary to take a step all the way back in the procedure to data evaluation.

It may be found that the test set does not predict well but the data in the test set may still be model members based on being inside the DModX. This would indicate new information and the test set should be added to the original data set. Consequently, the model should be re-run and the newly formed data set needs to be pre-processed. Once a model is found adequate for its purpose, the next step is to use it in-line with the process.

It should be noted that for a model of a manufacturing process, a one-time validation is never sufficient. There may be time dependent changes in the process that the model may not have been calibrated against. Such changes may be seasonal changes in the raw material used or temperature changes within the process or even machinery wear. One way to ensure the relevance of the model is to continuously withdraw samples for independent test sets. Then on a regular basis, the model should be monitored using MSPC to determine if it is still valid. 


\subsection{Drying Process Monitoring - MSPC}

A process is considered to be in a state of statistical control when key process variables remain close to their target values. The method for monitoring the process to confirm it is in control consists of using one of several statistical process control tools. One is the Shewhart control chart where one process variable is monitored at a time; however monitoring five variables one at a time is difficult for most operators to do. Another method is using multivariate statistics such as scores $(\mathrm{t})$ or Hotelling $\mathrm{T}^{2}-$ from a PCA or PLS model - and then use a Shewhart chart or an EWMA.

A concern with continuous processes is autocorrelation, where the value of an observation is dependent on the value of the observation taken or recorded before it. This impacts the variation or standard deviation as it is reduced in magnitude, thus making the Shewhart chart control limits tighter and the occurrence of false special cause increases (Noffsinger and Anderson 2002, Appendix B). The problem with autocorrelation can be offset using ARIMA type models for univariate monitoring (Appendix C).

When monitoring multivariate continuous processes with multivariate statistics such as t-scores or Hotelling $\mathrm{T}^{2}$ plotted on a Shewhart or EWMA chart, the intent is to monitor changes in the correlation structure of the variables. 


\section{THE OSB PROCESS FLOW MODEL}

"Every significant break through is first a break with tradition, old ways of thinking." Anonymous

\subsection{OSB Mill Process Flow - Key Process Variables}

Snee (2002) discussed four types of models. The OSB Mill Process Flow model is the first of Snee's models that defines the key variables or processing steps and their effects - positive and negative. The typical OSB manufacturing process has seven processing steps with multiple sub-steps. Each processing step has key variables that impact the productivity and product quality / properties.

The OSB Mill Process Flow Model is a flow chart / diagram focused on the key variables in each processing step. Table 5.1 is the OSB Mill Process Flow model that shows each of the "Processing Steps" and in parentheses the desired outcome for each step, e.g. "fresh clean logs". There is a "Process Control" column that lists items that should be controlled to ensure a reliable processing step that meets the desired outcome. If the process control items should vary then the "Consequences" column lists the effect including the impact on subsequent processing steps and product quality/properties. 
Table 5.1 OSB Mill Process Flow Model

\section{PROCESSING STEPS PROCESS CONTROL}

\section{LOG PREPARATION (Fresh, Clean Logs)}

Log Storage

Log Conditioning

Debarking

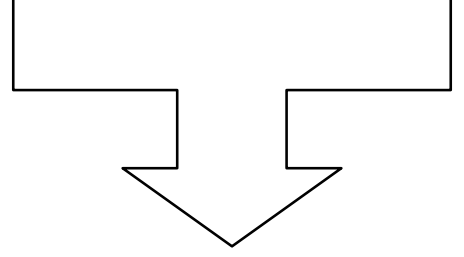

Inventory Rotation

Water Temperature and Dwell

Flail Chains and Feed Rate

\section{CONSEQUENCE}

Dried Out Logs

Mud and Grit Covered Logs

Poorly Debarked

Fines (-3/16") Increase

More Fines $=$ More Resin

Process Steps Impacted:

Stranding- mud covered logs causes dull knives

Screening - fines from dried out logs and grit

Drying - fines

Blending - fines

Properties Impacted:

Lower Bond Performance - Fines
Knife Projection \& Advance Time Splitter Bar Angle

Scoring Tips

Conveyor Filling

Screen mesh and angle

Bin Loading and \% Full
Strand Thickness - mean and Sd Strand Width variation

Strand Length

Fines (Logs rolling)

Fines

Process Steps Impacted:

Drying - Surging

Blending

Forming

Properties Impacted:

Thickness Swell - thickness Sd Surface roughness - wide strands Lower Bond Performance - fines Lower Post Flex / Stiffness short strands 
PROCESSING STEPS (continued)

\section{DRYING}

(Consistent Moisture

Content)

Heat Source

Weight Scales

Infeed Moisture

Outfeed Moisture

Dry Screening

Dry Strand Storage
PROCESS CONTROL (continued)

Fuel Preparation

Calibration 1

Calibration

Calibration

Mesh Opening and Angle

Bin Loading and \% Fill

\section{CONSEQUENCES}

(continued)
Dryer deluges - sparklers

Wet or Overdried - poor control Wet or Overdried - poor control Wet or Overdried - poor control Fines

Process Steps Impacted:

Blending - Surging w/ poor coverage

Blending - resin "wash in" and "wash out"

Forming - weight control Pressing - "blows" and or longer press cycle

Properties Impacted:

None directly impacted but can expect 1) poor bond performance due to moisture related blending issues, 2) poor strength properties in general due to forming issues due to weight control, and 3) panel thickness variation.

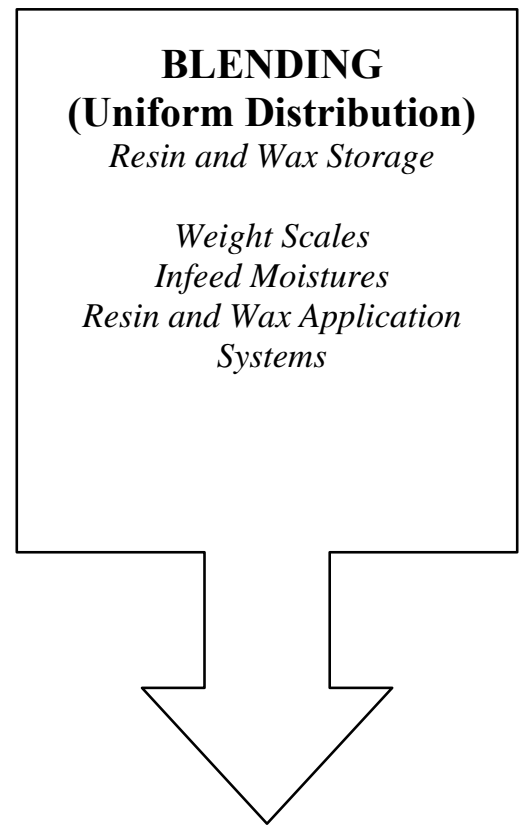

Storage Life and Rotation

Calibration

Calibration

Atomizer RPM

Blender RPM \& Angle
Increased viscosity - poor coverage

Incorrect application rate

Incorrect application rate

Too finely atomized poor coverage - pneumatics sucks out

Too coarsely atomized - poor coverage

Furnish does not get over resin boom - poor coverage

Process Steps Impacted:

Pressing - "blows" and or

longer press cycle

Finishing - edge density

Properties Impacted:

Bond Performance and Internal Bond - resin distribution 
PROCESSING STEPS (continued)

FORMING (Uniform Weight Distribution - Machine and Cross Direction)

Forming Bins

Deflector Plates

Weight Scales

Moisture

Magnets

Mat Trim Saws
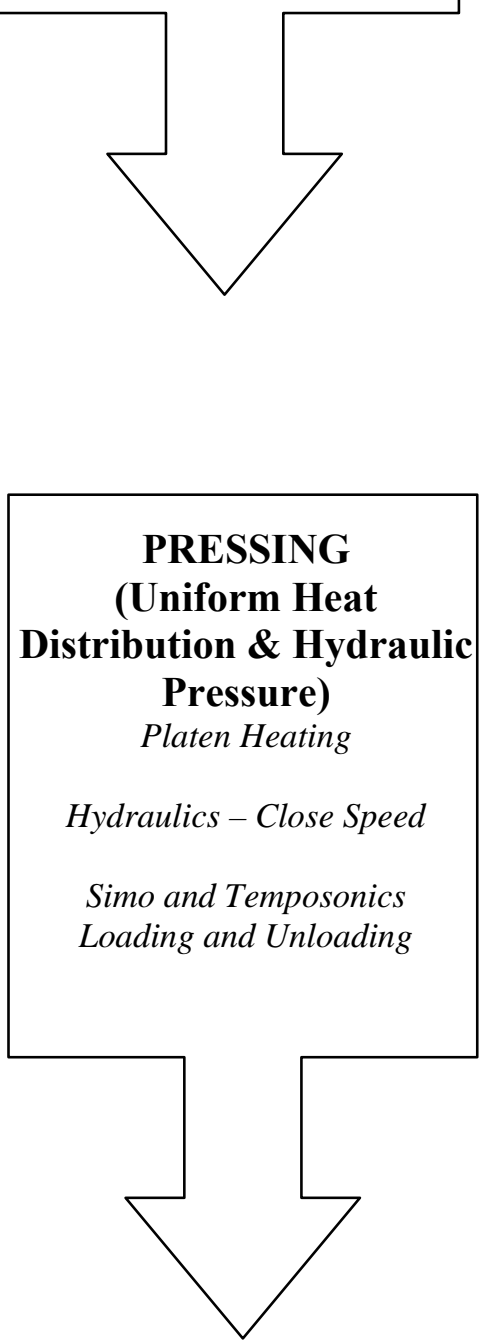

\section{PROCESS CONTROL (continued)}

CONSEQUENCES

(continued)

\author{
Bin Loading and \% Full \\ Angle \\ Calibration \\ Calibration \\ Hold down pressure and \\ sharpness \\ Cross Direction Weight \\ Cross Direction Weight \\ Machine Direction Weight and \\ Surface to Core Ratio \\ Machine Direction Weight \\ Metal in Press - Indent \\ Edge Density - Voids \\ Process Steps Impacted: \\ Pressing - "blows" and or \\ longer press cycle \\ Pressing - wedged mats \\ Finishing - edge voids \\ Properties Impacted: \\ Poor strength properties in \\ general due to forming weight \\ control issues \\ Panel thickness variation.
}

Hot Oil Temperature and Circulation

Press Design and Pressures

Calibration
Resin curing variation

Vertical Density Profile

Pre-cure if slow close

Mat thickness variation

Process Steps Impacted:

Pressing - "blows" and or longer press cycle

Finishing-panel thickness

Properties Impacted:

Flexure Properties if Vertical Density Profile too flat - slow close

Fastener Withdrawal if Vertical Density Profile is too steep fast close

Bond Performance and IB if poor heat distribution 
PROCESSING STEPS (continued)

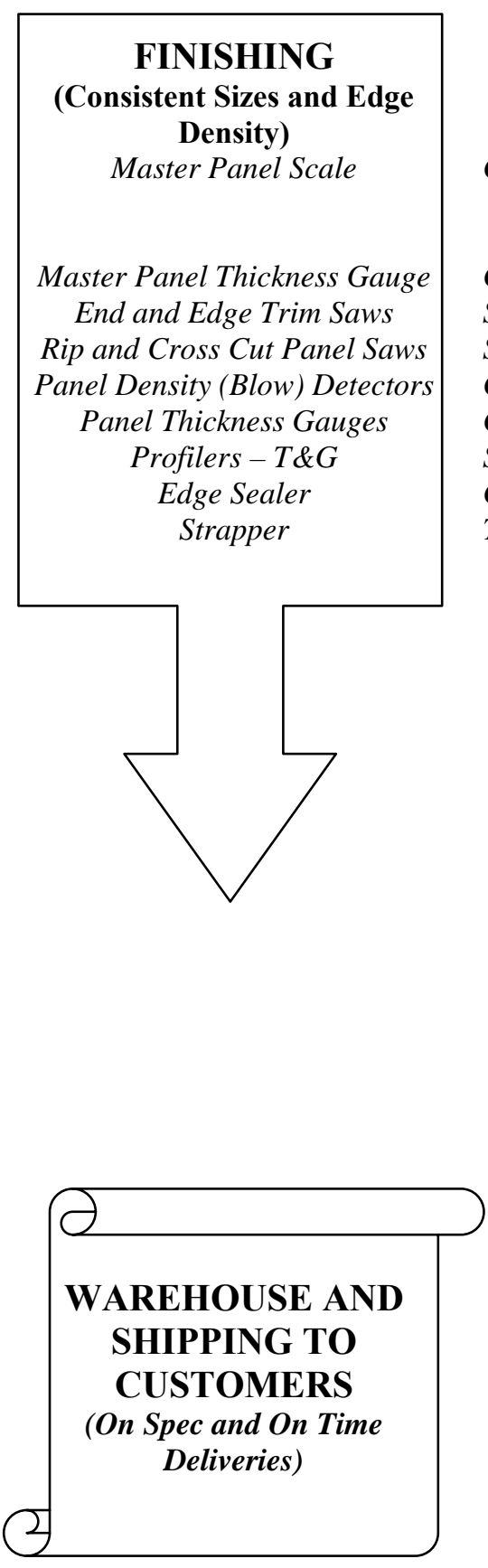

PROCESS CONTROL (continued)

Calibration

Calibration

Sharpness

Sharpness

Calibration

Calibration

Sharpness

Coverage in wet mil

Tension

\section{CONSEQUENCES (continued)}

\author{
Underweight panel - strength \\ properties \\ Overweight panel - waste \\ Grade out \\ Edge Voids \\ Edge Voids \\ Blown panels in finished units \\ Grade out \\ Poor fit \\ Edge Swell \\ Too much tension - T\&G \\ crushed while too little and units \\ come apart.
}

Process Steps Impacted:

Shipping and Customers with late deliveries if grade out is below plan

Properties Impacted:

Strength properties if weight gets low

Thickness swell if not enough edge sealer

Blown panels if not caught will have low properties

Edge voids can affect flexure properties. 


\section{THE OSB Mill Model \\ "Quality is Job One” Ford Motor Company}

The OSB Mill model is a Microsoft Excel ${ }^{\circledR}-$ based computer program developed as: 1) a management tool to study "what if" situations and to appreciate the "trade-offs" in the OSB processing steps, and 2) a mass balance program to smooth out process flow. The model is a spreadsheet representation of the OSB production process that "allows for far-ranging inferences — such that would be impossible with data alone” (Tal 2001).

Three major model revisions have occurred over the past nine years. The model was initially developed as a teaching tool by the author during mill start up in 1995 . It was designed to help mill leadership understand the basic material volumes necessary for the different product thickness' and press cycles. The model then evolved in 2001 into an operational tool to increase the awareness of the mass balance required for various press cycles and run speeds. Finally in 2003, the model became a management tool for answering "what if" questions around the various process steps. For example what if recovery is increased by incorporating more fines in the board thus reducing wood costs per msf, how much more resin can be used to offset the fines without increasing overall costs? How much oak related strander downtime is "acceptable" when the number of knife changes increases when processing oak?

The 1995 model version was initially developed from a concept authored by Bill Boehner when he was the Technical Manager at Weyerhaeuser's Grayling, MI OSB plant. The basic principle was to begin with the finished panel and work "backwards" removing resin and wax and adding back in moisture. This entailed the finishing, pressing, forming and blending processing steps. The basic concept over the past eight years has expanded to include drying followed by stranding, screening and debarking, 
and most recently adding in product mix, grade out, and cost; thus providing an entire mill model.

Model features such as the out of dryer moisture content for various resin levels is attributed to Dave Schrock with Georgia Pacific Resin Inc. who was helpful in implementing mass balance charts for the WonderWare ${ }^{\circledR}$ program at the plant. The product mix, grade out, and raw material costs for each product are based on concepts from annual financial planning.

The OSB Mill Model can be used in multiple ways. It can be used for mass balance to provide operations with assistance on how to run the process - when to slow down the dryers or when to speed up the formline. The model also has application in answering "what if" questions and helping answer trade-off questions. In the next several sections examples are given for the various mill model applications.

\subsection{Mass Balance}

The model was used in a rate surge strategy for "smoothing" out the process flow. The idea was to run to the bottleneck. The mill model was used to calculate the target weights/mass for the various processing steps based on pressed production. This was presented along with actual weights in the form of time series plots to the control room operators. As many as seven plots were projected on a wall in the control room. The plots showed the target weights and on the same plot the actual weight. This was done for each of the processing steps. This provided a quick, visual check on how the process had been running over say the last two hours. It also provided a quick check to see if the current target mass was balanced with the actual mass being processed. Figure 6.1 is one of the HMI screens projected on the wall that was used for the drying processing step. 


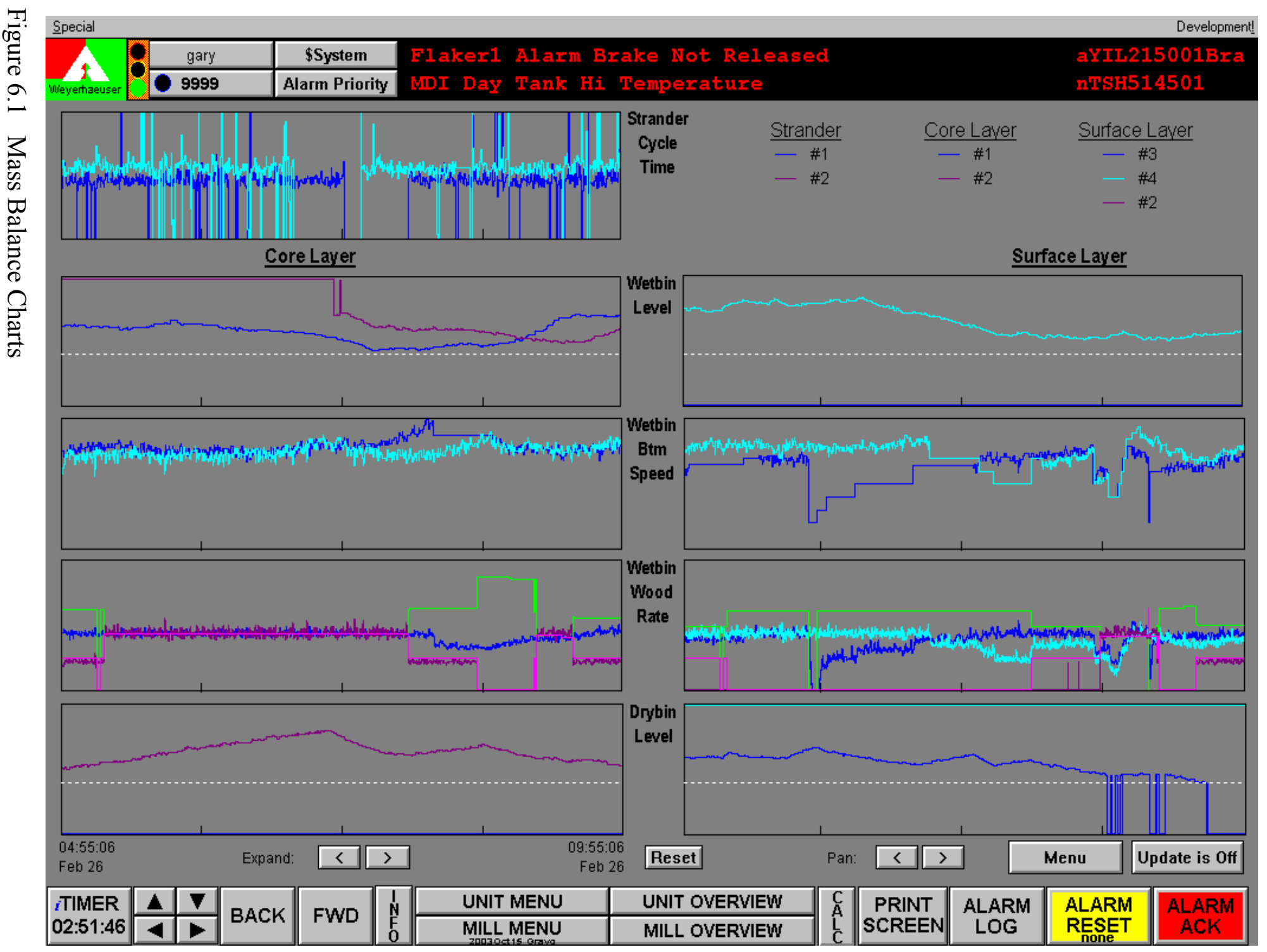




\subsection{Management Tool - "What if" and Trade-offs}

Knowing the trade-offs and getting answers to "what if" questions improve the quality of decisions and hopefully the profitability of the operation. The examples in the next several sections show the application of the mill model as a management tool and are examples of questions often asked by OSB operational personnel.

The goal of every operation is to safely run as much quality production as possible so that the operation can keep costs down. As the product line matures and becomes an indistinguishable commodity product, the mill will have to continue to provide a good return on investment to the stakeholders to stay in business. So asking what if questions and understanding the trade-offs may help the operation to continually improve, and permit it to stay in business.

\subsubsection{Strander Knife Changes vs. Advance Time}

OSB mills typically have problems during winter with frozen logs and producing enough strands to balance the process. Consequently the strategy is to run to the bottleneck and during winter it is the strander. It had become an accepted practice to increase knife projection and to slow down the carriage advance time, as it is believed better knife life occurs with fewer knife changes resulting in more strand production.

The mill model was used to study strander production to determine what effect reducing strander carriage advance times would have on first, strand production and second, with increased strand production how many knife changes could occur before the additional production is offset by the additional knife changes. There is a belief that if the carriage advance time is too short, knives will dull quicker. 
There were several assumptions that had to be made before the model could be used. The first was the species mix so a weighted average density of the mix could be determined. The second was the strand chamber fill ratio and the void space in the conveyor feeding the strander. A series of carriage advance times were then entered in the model, first with no knife changes and then with one, two, three and four to arrive at the strander production levels at various advance times and knife changes. This information was transferred to MINITAB ${ }^{\circledR}$ and a contour plot developed. The plot is shown in Figure 6.2.

For this particular study, the mill's carriage advance time was set at 16 seconds and the number of knife changes had been averaging 1.5 per strander per shift so far this season. The strand production was around 192,000 pounds per hour as shown by the vertical arrow in the figure. "What If" the advance time was reduced to 15 seconds and if there were no additional knife changes what would the strand production be? As shown in the plot it would increase to 200,000 as shown by the horizontal arrow.

The Continuous Improvement (CI) task team wanted to experiment with running faster carriage advance times but wanted some idea about trade-offs with knife changes. The mill model provided the information and is shown in the same figure as before. The advance time could be reduced to 14.5 seconds, and if the number of knife changes increased to three, the same production would occur as before. Or the advance time could be reduced to 13.5 seconds, and if there were less than four knife changes, the same or more production would result. 


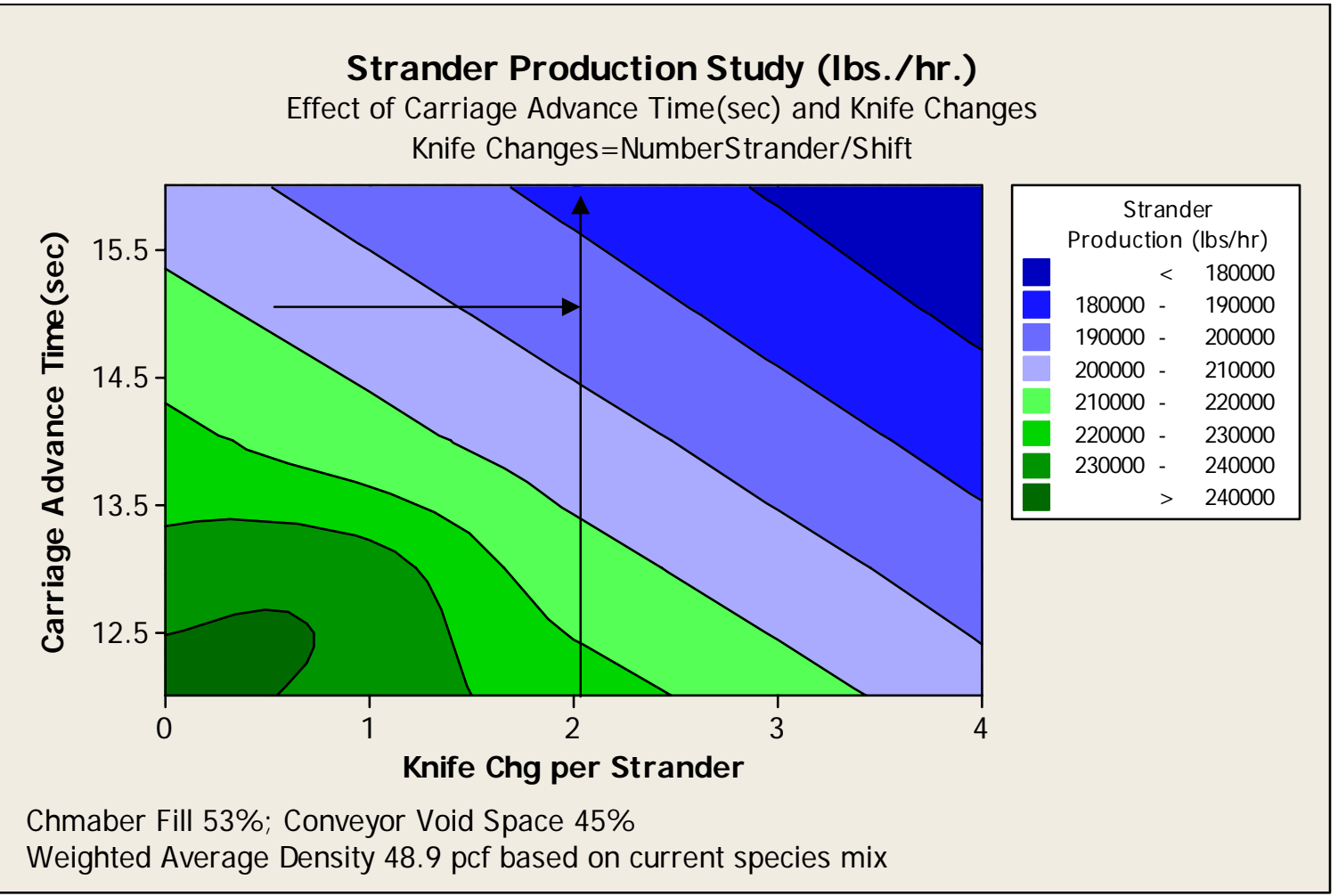

Figure 6.2 Strander Production Study Knife Changes vs. Carriage Advance Time and Their Affect on Production

\subsubsection{Strander Fines}

Another question that is frequently heard in OSB facilities is what is the trade-off with running more cuts or strokes at the strander even if it means making more fines? Every OSB manager who wants to get just a little more "production" asks this question, but they also want to know "what is the cost?" By knowing or "simulating" the percentage of fines at each level of stroke count the mill model can help answer the question about cost.

The fines content at each stroke count beginning with 100 strokes and going to 1600 strokes in intervals of 300 strokes were collected. The data were entered in the 
model and the wood cost determined from the model. Figure 6.3 shows escalating costs after about 1000 strokes.

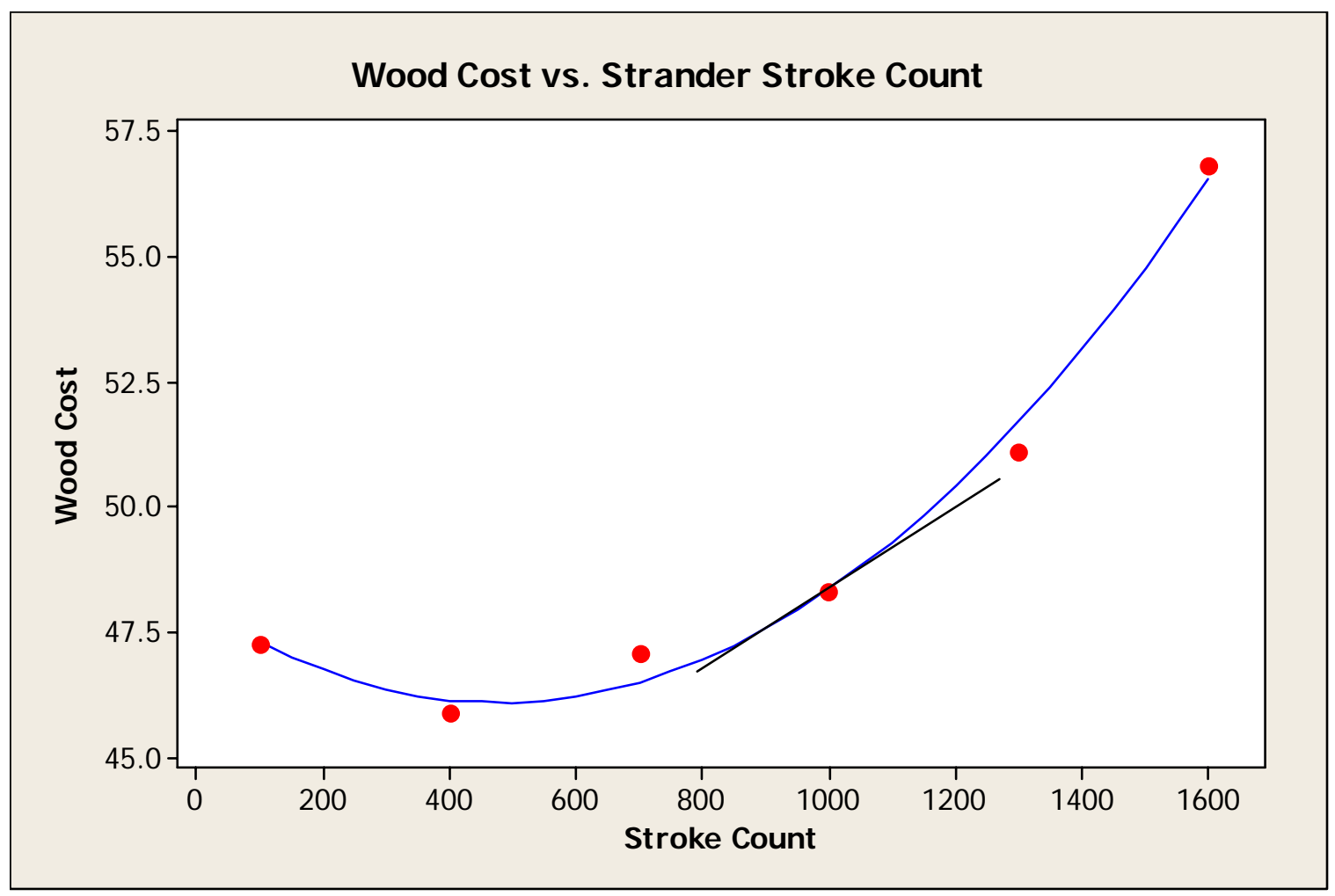

Figure 6.3 Fines vs. Stroke Count and Effect on Wood Cost

Another way to consider the trade-off question on fines is with "fines vs. recovery". Several fines content levels were entered in the model, and with all other variables held constant, the recovery, as measured by panel volume to ton of wood or msf (3/8's) per ton, was determined. This relationship is shown in Table 6.1. As the strander fines level increased the recovery was reduced with wood costs increased. Using the mill model again, an assumed screening efficiency was entered in the model input recipe page. This gave the "fines in the board", i.e. the amount of fines the screens could not screen out. This is shown in Table 6.1. It shows that as strander fines increase, more fines get mixed into the board. Typically what is done to offset an increase in fines is to 
increase resin addition rates. So as strander fines increase recovery decreases and wood costs increase as do resin costs.

Table 6.1 Strander Fines vs. Recovery Wood Cost and Fines in Board

\begin{tabular}{cccc}
\hline \hline $\begin{array}{c}\text { Strander } \\
\text { Fines } \%\end{array}$ & $\begin{array}{c}\text { Recovery } \\
\text { MSF(3/8's)/Ton }\end{array}$ & $\begin{array}{c}\text { Wood Cost } \\
\text { \$MSF(3/8's) }\end{array}$ & $\begin{array}{c}\text { Fines in Board } \\
\%\end{array}$ \\
\hline 20 & 0.730 & 47.92 & 14.80 \\
20 & 0.720 & 48.58 & 13.70 \\
20 & 0.710 & 49.26 & 12.50 \\
\hline 25 & 0.719 & 48.67 & 18.80 \\
25 & 0.706 & 49.54 & 17.50 \\
25 & 0.694 & 50.45 & 16.25 \\
\hline 30 & 0.708 & 49.45 & 22.00 \\
30 & 0.692 & 50.54 & 20.50 \\
30 & 0.677 & 51.71 & 19.00 \\
\hline 35 & 0.696 & 50.27 & 26.00 \\
35 & 0.678 & 51.61 & 24.00 \\
35 & 0.660 & 53.06 & 22.00 \\
\hline 40 & 0.685 & 51.12 & 29.50 \\
40 & 0.667 & 52.73 & 27.00 \\
40 & 0.642 & 54.52 & 24.40 \\
\hline \hline
\end{tabular}

\subsubsection{Panel Density vs. Resin}

A question sometimes asked by management is, "what about reducing panel density and adding resin in its place?" This question might arise if wood costs are increasing while resin costs are flat. To answer this question the OSB Mill Model was used to determine the cost implications.

First the current formulation - panel density and resin rates - were input in the mill model to get the total raw material cost for the current formulation. Next the desired panel density level and a substituted resin level were entered to get a raw material cost. With several iterations of adjusting the resin levels, a new formulation is obtained at the same raw material costs as the original formulation but with more resin and the desired 
lower panel density. This gives resin and panel density factor levels for a DOE to begin testing to confirm board quality can be maintained.

For example, say the current product formulation is $45.8 \mathrm{pcf}$ panel density with resin rates of $4.25 \% \mathrm{LPF}$ and $3.0 \%$ pMDI. The raw material cost would be $\$ 81.98$ per msf (3/8's). If the desired density is $44.8 \mathrm{pcf}$, through several iterations, the new resin rates are obtained at $4.65 \% \mathrm{LPF}$ and $3.21 \%$ pMDI with same raw material cost. 


\section{DRYING MODEL DEVELOPMENT, VALIDATION, AND PREDICTION}

"Any model is at best a useful fiction - there never was, or ever will be, an exactly normal distribution or an exact linear relationship. Nevertheless enormous progress has been made by entertaining such fictions and using then as approximations." George Box

Efficient OSB drying systems reduce strand moisture content variation and deliver consistent panel densities and panel thicknesses. Efficient drying operations occur with the knowledge of what process variables affect strand moisture content and how to adjust them using process control to minimize the variation. The economic incentive for improving efficiency and minimizing process variation is driven by reducing costs and increasing sales realizations with the ultimate goal of increasing the return on investment to the stakeholders.

Reduced costs are possible by reducing panel density variation that in turn increases recovery and reduces wood costs per msf (3/8ths). Honda (1984) reported with medium density fiberboard (MDF) by reducing the standard deviation of the fiber moisture content from $1.3 \%$ to $0.2 \%$, the panel thickness variation was reduced by $18.1 \%$; while the panel weight variation was reduced by $8.5 \%$; with panel density reduced by $16 \%$.

Sales realizations are increased by improving on-grade panel production through better control of panel thickness. Ducharme (2003) reported for OSB that for each 1.5\% change in moisture content, the panel thickness changed by 0.005 "; while, Honda (1984) indicated MDF thickness variation reduction was possible by reducing moisture variation.

Honda (1984) described the particle / fiber drying control process as a simple proportional integral derivative (PID) control loop or as complex as a Smith Predictor 
loop. The PID control loop takes the measured value, outlet temperature, and subtracts it from the set point or target. This difference or "error" is multiplied by the gain or proportional term to get the controller output moving in the right direction. If the upset or error is present for a period of time, the integral term will integrate the error and add it to the proportional term in an effort to null out the error or offset. When rapid changes in the process occur, the derivative term limits the rate of change in the controller output.

\subsection{Data Analysis and Pre-Processing}

The initial variables considered for this research included 11 for the heat source and 18 for the dryer. Table 7.1 contains a list of the variables and explains each of their functions. The design of experiments was discussed in more detail in Chapter 4 section 4.2.6. The two variables used in the design of experiments were outlet temperature and

feed rate. The design was a simple $2^{2}$ replicated twice with outlet temperature levels of $176^{\circ} \mathrm{F}$ and $181^{\circ} \mathrm{F}$ and feed rates levels of 39,000 pounds and 42,000 pounds.

The data were reviewed or pre-processed using SIMCA-P (Umetrics 2001). The variables were put in classes based on the run number as shown in Table 7.2. They were then plotted in time series to look for obvious data errors such as a zero-value or outrageously high value due to a faulty sensor or device. No errors were observed. 


\section{Table 7.1 Dryer Model Study Variables}

\begin{tabular}{|c|c|}
\hline Variable & Function / Purpose \\
\hline \multicolumn{2}{|c|}{ Heat Source - GTS Energy, Inc \# 1} \\
\hline *Firing Rate & $\begin{array}{l}\text { Control variable for combustion temperature and the primary, secondary and re- } \\
\text { circulation air system to control combustion temperatures }\end{array}$ \\
\hline *Blend Air Temperature & $\begin{array}{l}\text { Temperature from heat source that allows modulating the combustion temperature } \\
\text { prior to the dryers }\end{array}$ \\
\hline $\begin{array}{l}\text { Blend Air Temperature Set } \\
\text { Point }\end{array}$ & $\begin{array}{l}\text { Set point for bend air temperature chamber. If the temperature gets too high press } \\
\text { vent air can be redirected to modulate }\end{array}$ \\
\hline $\begin{array}{lr}\text { Blend Air } & \text { Temperature } \\
\text { (Auto/Manual) }\end{array}$ & Variable to monitor whether the blend air is operating in PID control or manually. \\
\hline $\begin{array}{ll}\text { *Combustion } & \text { Air } \\
\text { Temperature } & \end{array}$ & The gas temperature located above the grate sections \\
\hline $\begin{array}{l}\text { Combustion Air Temperature } \\
\text { Set Point }\end{array}$ & Set point for combustion temperature - typically set at $1800^{\circ} \mathrm{F}$ \\
\hline $\begin{array}{l}\text { Combustion Air Temperature } \\
\text { (Auto/Manual) }\end{array}$ & $\begin{array}{l}\text { Variable to monitor whether the combustion air is operating in PID control or } \\
\text { manually }\end{array}$ \\
\hline *Flue Gas Temperature & $\begin{array}{l}\text { Temperature measured in the duct work going to the heat exchanger and the blend } \\
\text { air chamber }\end{array}$ \\
\hline Primary Air Damper \#2 & Adjusts the amount of below grate air into the $3^{\text {rd }}$ and $4^{\text {th }}$ grate sections \\
\hline Secondary Air Damper & $\begin{array}{l}\text { Secondary air is used to control the above grate combustion and to control } \\
\text { "sparklers" entering the duct system and getting into the dryer where fire may be } \\
\text { the result }\end{array}$ \\
\hline Re-circulation Air Damper & $\begin{array}{l}\text { Re-circulation air is used to reduce combustion as it is oxygen deficient and to } \\
\text { minimize } \mathrm{NO}_{\mathrm{x}}\end{array}$ \\
\hline \multicolumn{2}{|l|}{ Dryer 1} \\
\hline Bin Level, Green & $\begin{array}{l}\text { Level of the bin measured with a laser, is a percentage of the length the bin is filled } \\
\text { with furnish }\end{array}$ \\
\hline Green Bin, Bottom Belt Speed & Controlled variable for Feed Rate \\
\hline Bin Level, Dry & $\begin{array}{l}\text { Level of the bin measured with a laser, is a percentage of the length the bin is filled } \\
\text { with furnish - Not operational during the trial }\end{array}$ \\
\hline Dry Bin Bottom Belt Speed & Controlled variable for blender demand \\
\hline Inlet Moisture Content & In coming furnish green or wet moisture content (OD basis) \\
\hline *Outlet Moisture Content & Dried furnish coming from the dryer (OD basis) \\
\hline *Inlet Temperature & $\begin{array}{l}\text { Temperature of the air going into the dryer as measured prior to the down chute for } \\
\text { the furnish, on the heat source side }\end{array}$ \\
\hline *Hot Air Out or Heart Valve & The damper position for modulating the outlet temperature \\
\hline *Outlet Temperature & $\begin{array}{l}\text { Outlet temperature as measured on the outlet of the dry drum prior to the high } \\
\text { efficiency cyclones }\end{array}$ \\
\hline $\begin{array}{l}\text { *Outlet Temperature Set } \\
\text { Point }\end{array}$ & Set point for the outlet temperature -typically set at $170^{\circ} \mathrm{F}$ to $180^{\circ} \mathrm{F}$ \\
\hline $\begin{array}{ll}\begin{array}{l}\text { Outlet } \\
\text { (Auto/Manual) }\end{array} & \text { Temperature } \\
\end{array}$ & $\begin{array}{l}\text { Variable to monitor whether the outlet temperature is operating in PID control or } \\
\text { manually }\end{array}$ \\
\hline *Feed Rate or Weight Scale & Weight of furnish going across the Bertholdt nuclear scale into the dryer \\
\hline $\begin{array}{l}\text { *Feed Rate or Weight Scale Set } \\
\text { Point }\end{array}$ & Set point for feed rate variable \\
\hline $\begin{array}{l}\text { Feed Rate or Weight Scale } \\
\text { (Auto/Manual) }\end{array}$ & Variable to monitor whether the feed rate is operating in PID control or manually \\
\hline *ID Fan Amperage & $\begin{array}{l}\text { The amperage draw for the induced draft fan on the outlet of the driers located after } \\
\text { the high efficiency cyclones }\end{array}$ \\
\hline ID Fan Amperage Set Point & Set point for ID Fan amps - typically set at 80 amps \\
\hline $\begin{array}{l}\text { ID Fan Amperage } \\
\text { (Auto/Manual) }\end{array}$ & Variable to monitor whether the ID Fan is operating in PID control or manually \\
\hline $\begin{array}{l}\text { *Static Pressure, Dryer Outlet } \\
\text { or Outlet Pressure }\end{array}$ & $\begin{array}{l}\text { The static air pressure measured on the outlet of the dryer. The value becomes } \\
\text { larger, more negative, as more furnish is dried. }\end{array}$ \\
\hline
\end{tabular}


Table 7.2 Treatments Run Order Classes and Sample Size after the Lag

\begin{tabular}{ccccc}
\hline \hline Run \# / Class & Feed Rate Set Point & Outlet Temp Set Point & $\mathrm{n}$ & Obs \#'s \\
1 & 42,000 & 181 & 44 & $1-44$ \\
2 & 39,000 & 181 & 42 & $45-85$ \\
3 & 39,000 & 176 & 13 & $86-98$ \\
4 & 42,000 & 176 & 43 & $99-141$ \\
5 & 42,000 & 181 & 4 & $142-145$ \\
6 & 39,000 & 181 & 13 & $146-158$ \\
7 & 39,000 & 176 & 8 & $159-166$ \\
8 & 42,000 & 176 & 13 & $167-179$ \\
\hline
\end{tabular}

The green bin level, green bin bottom belt speed and dry bin bottom belt speed were dropped as they were controlled very tightly during the trial to minimize surging of the furnish going into the dryer. It was decided shortly before starting the trial to minimize the surging by allowing time, if needed, to build the bin level back to over $50 \%$ before each new treatment / run. This minimized the variation of these variables and they were subsequently dropped.

The primary air, re-circulation air and secondary air dampers were dropped due to little to no change by the controllers over the eight runs. It was believed they were malfunctioning during the trial. The Auto/Manual variables for Blend Air Temperature, Combustion Air Temperature, Outlet Temperature, Feed Rate, and ID Fan were all in automatic control during the trial so they were dropped. Set Points for Blend Air Temperature, Combustion Air Temperature and ID Fan were also dropped due to no change over the trials. Finally the dry bin level and inlet moisture content were dropped due to device failures. Eleven variables remained and are shown in bold type with an asterisk in Table 7.1. 
Normality for the variables was examined to determine if transformations were needed. Several of the variables were slightly skewed but no transformations were made. A quick summary of the key statistics on a few of the variables is shown in Table 7.3.

Table 7.3 Key Statistics

\begin{tabular}{llcccc}
\hline \hline Variable & $\mathrm{n}$ & Mean & Stand Dev & Maximum & Minimum \\
\hline Outlet MC, ${ }^{\circ}{ }^{\circ}$ & 113 & 5.4 & 0.5 & 6.5 & 4.1 \\
Outlet Temp ${ }^{\circ} \mathrm{F}$ & 113 & 180 & 3.4 & 187 & 171 \\
Inlet Temp, ${ }^{\circ} \mathrm{F}$ & 113 & 780 & 55 & 884 & 660 \\
Blend Air Temp, ${ }^{\circ} \mathrm{F}$ & 113 & 1231 & 99 & 1383 & 1044 \\
Combustion Temp, $^{\mathrm{o}} \mathrm{F}$ & 113 & 1850 & 69 & 1947 & 1666 \\
Flue Temp, ${ }^{\circ} \mathrm{F}$ & 113 & 1570 & 80 & 1746 & 1401 \\
\hline \hline
\end{tabular}

The variables were studied to see if lag transformations were needed as the sensors or devices for the variables were not located in close proximity to one another. For example, combustion temperature and flue gas temperature sensors were located in the heat source about 50 feet from the temperature sensor to the dryer infeed; while the outlet temperature sensor was about 750 feet further down stream. The data historian stored the scanned data using the same time stamp so when comparing the predictor variables to the response variable, lagging the variables might be required to obtain a good understanding of the relationships.

The cross correlation transformation routine in SIMCA-P was used to determine the necessary lag time to maximize the correlation between the outlet moisture content and the other variables such as combustion temperature, blend air temperature, inlet temperature, outlet pressure and outlet temperature.

The lagged time for outlet temperature from run 1 was Lag [2] or two minutes. The outlet temperature at time 15 minutes, for example, was not "seen" at the outlet moisture content meter until 2 minutes later or at time 17 minutes. The lagged amount 
was determined for all the variables using the data collected from each run. The lag times for all eight runs were averaged to determine the lag transformation to use in model development.

The lags were adjusted using outlet pressure as the base and not outlet MC. Outlet pressure was used because the cross correlation lag for outlet pressure with outlet moisture content was Lag [-6]. The average lag times for the eight runs are listed in Table 7.4. The heat source variables and inlet temperature were so close together it was decided for simplicity to just use one value. Each variable was lag transformed for each run using the lagged values with SIMCA-P.

Table 7.4 Variables Lagged Times

\begin{tabular}{lcc}
\hline \hline Variable & Average Lag Times & Lagged \\
\hline Outlet Pressure & 0 & 0 \\
ID Fan & 0 & 0 \\
Outlet MC & 6 & 6 \\
Weight Scale & 6 & 6 \\
Outlet Temperature & 7 & 7 \\
Inlet Temperature & 18 & 17 \\
HotAirOut & 19 & 17 \\
Fire Rate & 17 & 17 \\
Blend Air Temperature & 17 & 17 \\
Combustion Temperature & 18 & 17 \\
Flue Gas Temperature & 17 & 17 \\
\hline \hline
\end{tabular}

The training set for model development was a matrix 8 runs by 11 variables. The dryer outlet moisture content was the response variable, Y. The predictor variables, $\mathbf{X}$, were the other 10 variables.

The data were mean-centered and unit variance scaled to get all variables on "equal footing" and not have variables with large scale have greater leverage or influence in the model. 


\subsection{Model Development}

The objective of model development was to determine the variables or combination of variables that influence outlet moisture content levels so ultimately these variables can be controlled and the outlet moisture content variation can be reduced.

\subsubsection{Principal Component Analysis (PCA)}

PCA was conducted to study the behavior or relationships of the data. Table 7.5 shows each of the components and the contribution made in explaining the variation in the data set. $\mathrm{R}^{2} \mathrm{X}$ is fraction of the sum of squares (SS) of all the $\mathrm{X}$ 's explained by the current component or dimension. $\mathrm{Q}^{2}$ is the fraction of the remaining variation of the $\mathrm{X}^{\prime} \mathrm{s}$ that can be predicted by the current component. The first component explained $40.5 \%$ of the variation of the X's and predicted $22.6 \%$ of the variation of the X's

\section{Table 7.5 PCA-X Model}

\begin{tabular}{ccccc}
\hline \hline Comp(A) & $\mathrm{R}^{2} \mathrm{X}$ & $\mathrm{R}^{2} \mathrm{X}(\mathrm{Cum})$ & $\mathrm{Q}^{2}$ & $\mathrm{Q}^{2}$ (Cum) \\
\hline 1 & 0.405 & 0.405 & 0.226 & 0.226 \\
2 & 0.284 & 0.649 & 0.101 & 0.304 \\
3 & 0.181 & 0.830 & 0.334 & 0.537 \\
4 & 0.083 & 0.913 & 0.242 & 0.649 \\
\hline \hline
\end{tabular}

\subsubsection{Outliers}

The observations were examined to see if there were any outliers in the data set. An outlier is defined as an observation that is either extreme or does not fit the model well, but an outlier can also be very informative as it may be spanning a particular type of variability in the data set not modeled and in fact not be erroneous. To remove an outlier is a "judgment" call and left up to the experimenter.

The scores of $\mathrm{t}[1]$ vs. $\mathrm{t}$ [2] were plotted to look for outliers that might unduly influence and "force" a component to be developed due to its existence (Figure 7.1). Two 
observations in quadrant two, upper left, that were outside Hotelling $\mathrm{T}^{2}$ s $95 \%$ confidence limit ellipse indicating they might be "potential" outliers. On the other hand, they could easily have fallen in the $5 \%$ of the observations that might by all probability have fallen outside the ellipse since Hotelling $\mathrm{T}^{2}$, $\mathrm{s}$ ellipse is a $95 \%$ confidence limit. No observations were removed.

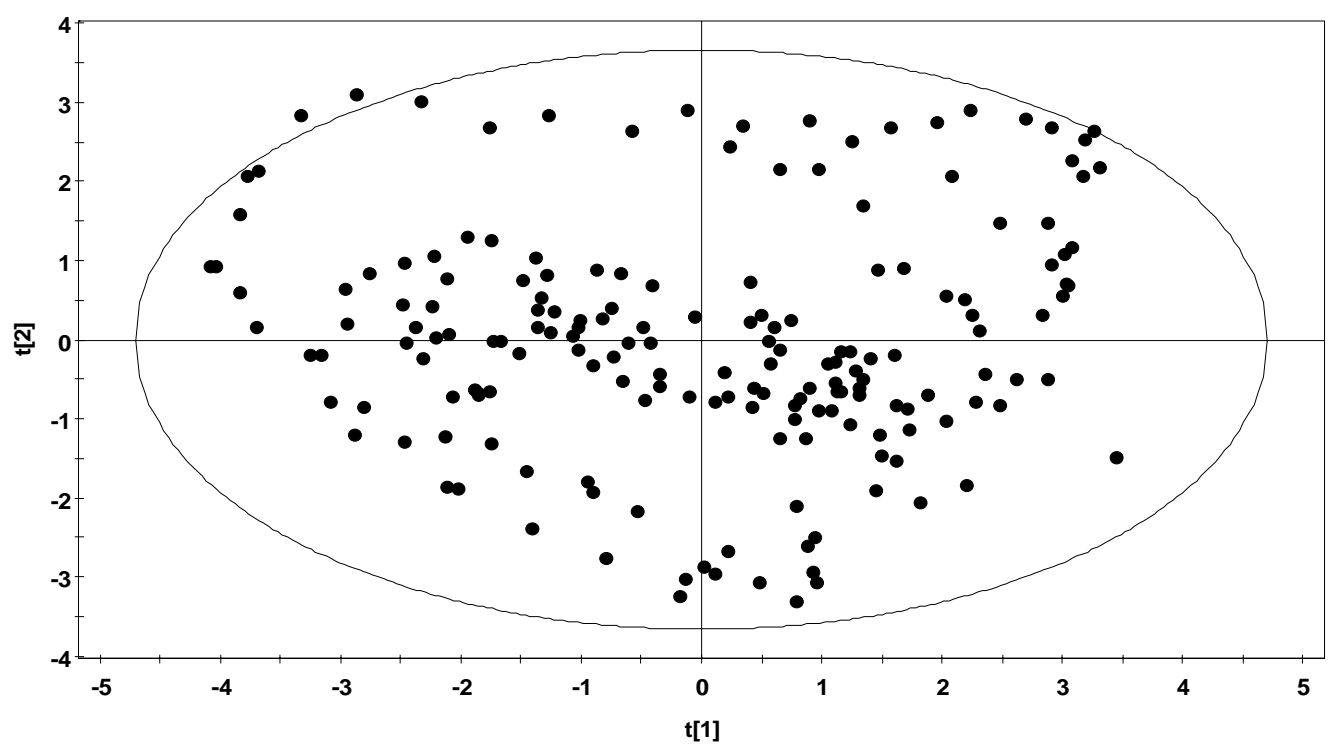

Figure 7.1 PCA-X for Dryer Model Development - Score Plot t[1]/t[2]

The observations that did not fit the model well were investigated using the DModX plot (Figure 7.2). There were three spikes in the data. This might have indicated outliers, either as deviations of the real data from the model or the model does not capture the $\mathrm{X}$-variation well, i.e. the underlying relationship of the data. While there were a few observations above the D-Crit (0.05), the number was no greater than chance, and with no serious or extreme departures no observations were removed. 


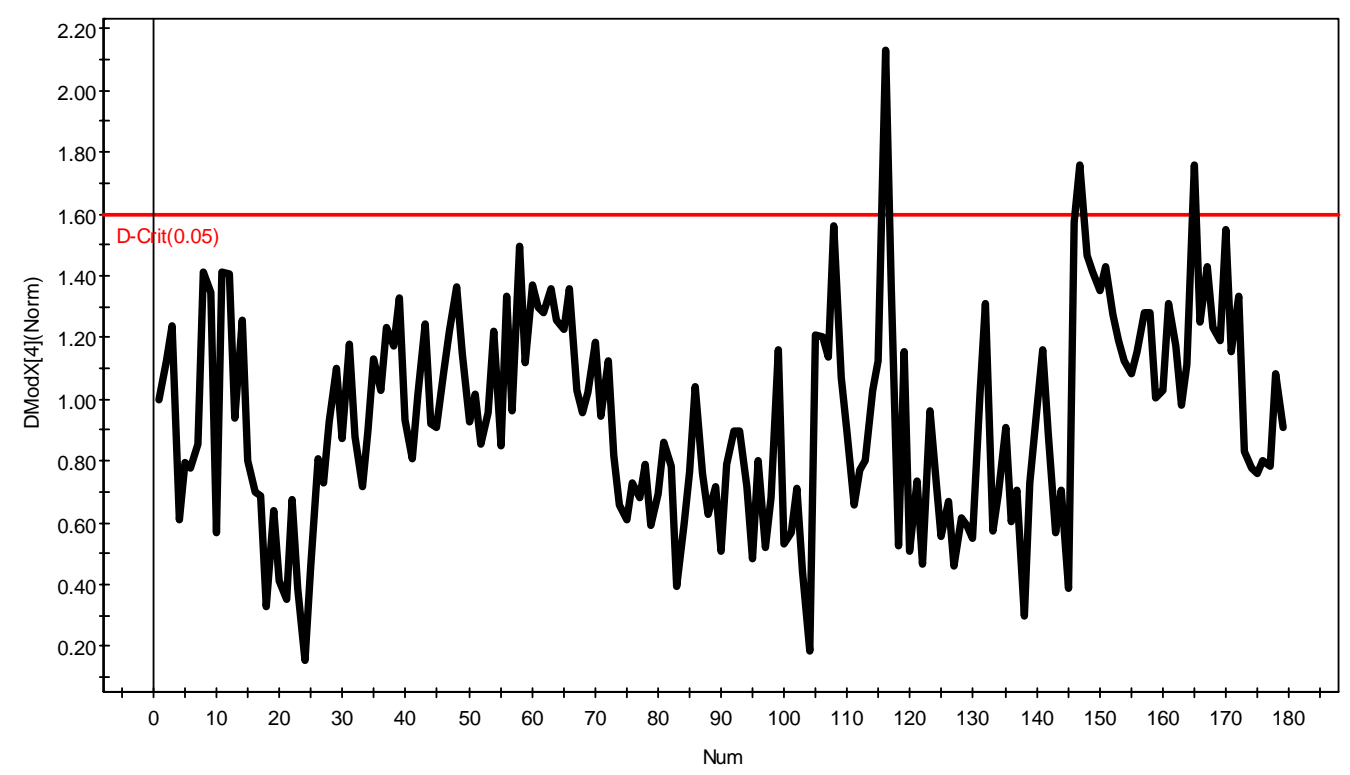

Figure 7.2 DModX for PCA-X.

\subsubsection{Observations - Scores}

A score for a particular observation is a linear combination of predictor $\mathbf{X}$ 's based on variable weights or loadings. A score is a new variable or latent variable that summarizes the original variables into one new value. Scores are projections to a low dimension window from high dimension $\mathrm{X}$ space. Points in two dimensional window, scores $\mathrm{t}[1]$ and $\mathrm{t}[2]$ that are close together may be similar; while those far apart are dissimilar. For example scores clustered in the same quadrant may be similar due to the linear combination of weights providing the same or similar score values.

The two dimensional score plot, Figure 7.3, for $\mathrm{t}[1]$ and $\mathrm{t}[2]$, shows the scores by treatments or classes. It was expected that the observations from each treatment from the design of experiment would be clustered together with the replicate treatment set clustered near by. The plot shows the treatments/classes clustered but several wander 
about. Both treatment/class \#2 and \#4 wandered about after being in a loose cluster. The movement indicates something in the process is changing during \#2 and \#4 runs. The discussion on what caused the drifting will be covered in more detail in section 7.2.1.4, Interpreting the Score Plot.
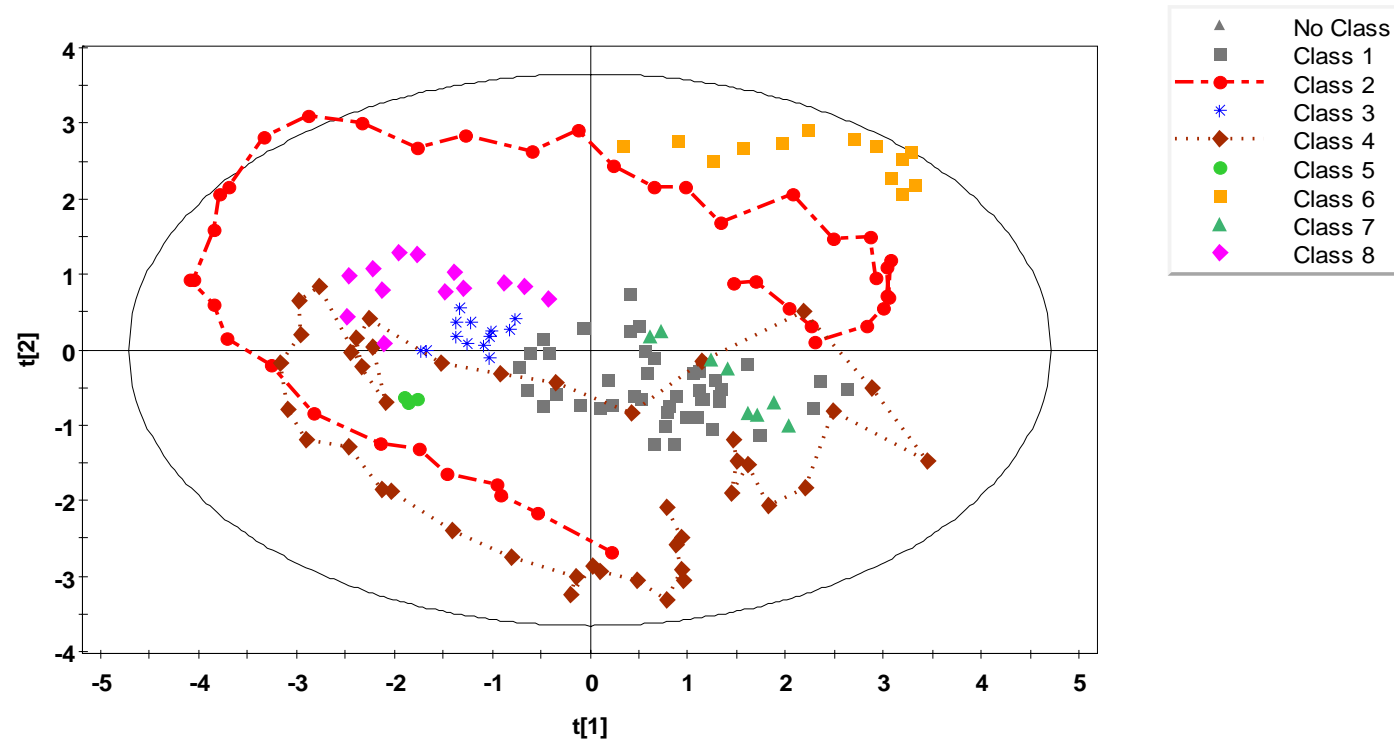

Figure 7.3 Score Plot $\mathrm{t} 1 / \mathrm{t} 12$ by Treatment/Class

\subsubsection{Variables - Loading}

The loadings or weights help to explain the scores plot by revealing which variables are responsible for the patterns seen among the observations. The loadings are the variable weights used in the linear combination of the observation that form the score.

The direction in the loading plot corresponds to the direction in the score plot. Looking at the two plots together helps to determine which variables are most influential in the linear combination that made the score. 
The two dimensional loading plot, Figure 7.4, shows the locations of the variables. Variables in the same quadrant, such as the combustion temperature and flue temperature, are positively correlated. The horizontal axis in Figure 7.4 is the first dimension or component and in the first component, combustion and flue temperature have loading values of -0.40 and -0.47 , respectively. This indicates they are negatively correlated with the first component but positively correlated with each other. In the second component the loading values are -0.20 and -0.22 indicating negative correlation with the second component, the vertical axis, but positively correlated with one another. Since they positively correlated with each other in both components, they are strongly positively correlated with each other.

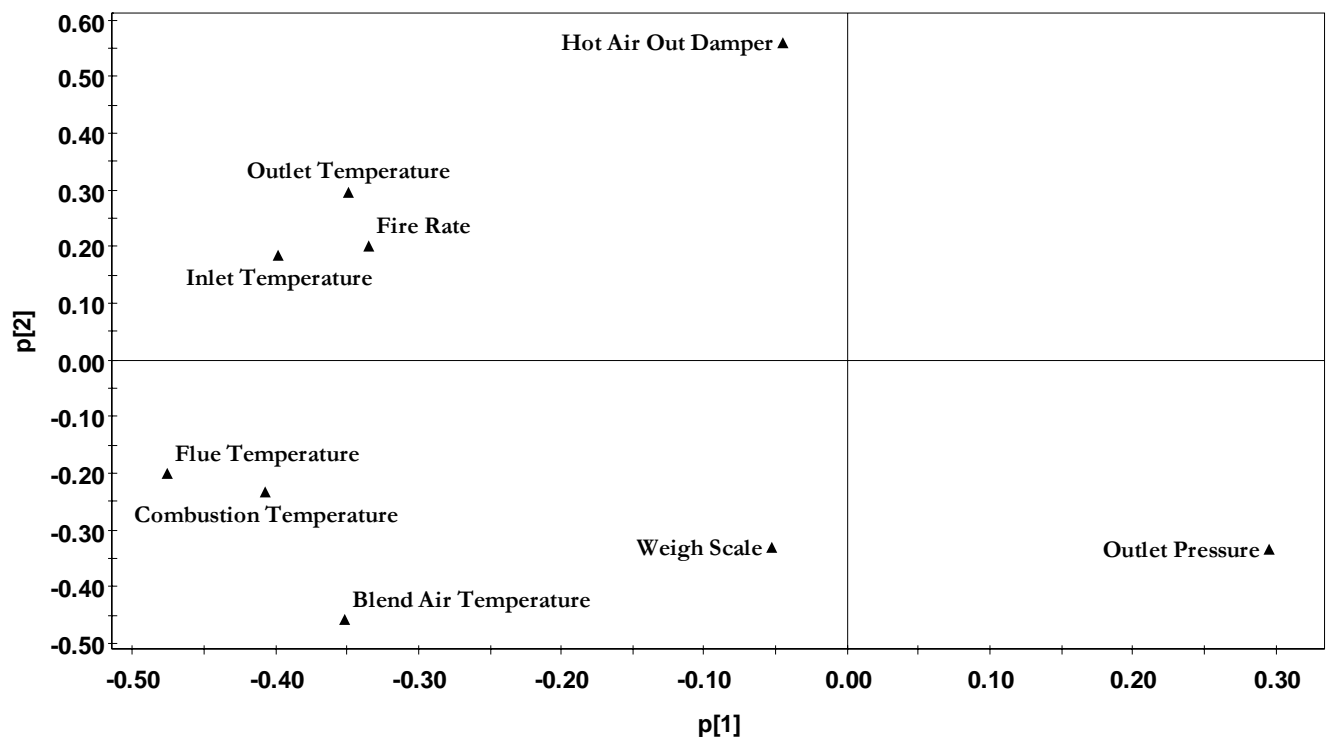

Figure 7.4 Loading Plot for PCA-X p[1]/p[2]

Variables in diagonally opposite quadrants, outlet temperature and outlet pressure, are negatively correlated to one another. The loading value for outlet temp with the first 
component is -0.34 ; while it is +0.30 for the outlet pressure indicating a negative correlation on to the other. With the second component, the outlet temp loading value is +0.30 and for the outlet pressure it is -0.35 , again negatively correlated with each other. Since they were negatively correlated in each component, they are strongly negatively correlated with each other in the loading plot.

Variables in quadrants 90 degrees to each other are weakly correlated either negatively or positively depending on the loadings.

In summary, the two dimensional loading plot, Figure 7.4, shows which variables are clustered together or far apart, which ones are on opposite corners of the plot, and which ones are far from or near the origin. This helps to see the correlation structure among the variables and their influence on the model. When variables are tightly clustered, they are positively correlated to one another. When variables are negatively correlated to one another, they are positioned on opposite sides of the plot origin in diagonally opposite quadrants. The further a variable is from the origin the stronger the influence that variable has on the model.

The first component is the projection of the weights on to the horizontal axis indicating the importance of each variable, that is, the larger the weight the more influential. It shows the process temperatures - heat source (Heat1BlndAirT, Heat1CombT, and Heat1FluT) and the dryer (Dry1InT and Dry1OutT) - on the left lower side of the plot indicating positive correlation between these variables. With the variables lagged, the increase in dryer temperatures occurs at the same time as the increase in the heat source temperatures. The first component might be characterized as process temperatures. 
The second component shows a mechanical relationship between the hot air out damper system and the blend air system albeit a negative one with the loading of +0.6 and a -0.45 for the two variable respectively (Figure 7.4 ). The damper opens to allow more hot air to flow to the dryer or closes to slow the air flow. When the damper opens, the blend air chamber temperature drops; and when the damper closes, the blend air chamber temperature increases. Component two might be classified as mechanical.

\subsubsection{Interpreting the Score Plot}

Studying the score plot (Figure 7.3) and loading plot (Figure 7.4) together is useful in determining which variables are responsible for the patterns seen among the observations in the score plot. There were two very interesting patterns with treatment \#4 and \#2. Both were predominately left to right patterns with some up and down variations included. Why?

Treatment \#4 started off as a small cluster in quadrant four and then started moving up and to the left into quadrant two. It then zigged back into quadrant three and then finally into quadrant four when the run ended. This reversing course pattern is due to cycling by the process temperatures more specifically the outlet and inlet temperatures. The inlet temperature cycled from $700^{\circ} \mathrm{F}$ to $870^{\circ} \mathrm{F}$ to $670^{\circ} \mathrm{F}$; while the outlet temperature cycled from $167^{\circ} \mathrm{F}$ to $187^{\circ} \mathrm{F}$ to $166^{\circ} \mathrm{F}$. The increasing temperatures caused the scores to move to quadrant two. Then when the temperatures started to drop the pattern reversed and went back to quadrant four where it began. It took a slightly southern route in the return through quadrant three due to the hot air out damper closing. The pattern in the beginning when the score traveled up into quadrant two was due to the hot air out damper increasing while the blend air temperature was decreasing. 
Treatment \#2 started out as a "loose" cluster and then began its walk about and headed to the second quadrant and finally wandering into the third quadrant. The loading plot indicates the process temperatures specifically the outlet and inlet temperatures "pulling" the observations out of the cluster as they increase in value and into second quadrant. The movement from the second to third quadrant is related to the mechanical dimension with blend air temperature increasing and the hot air out damper decreasing and causing the movement downward. The movement back across the first dimension into the fourth quadrant is again process temperatures related as both inlet and outlet temperatures dropped.

\subsubsection{Summary of PCA Analysis}

The PCA explained the variation in the data set by combining the relationships of the variables into the two dimensions entitled process temperatures and mechanical. The score patterns moving across the first dimension were influenced by inlet and outlet temperatures, process temperatures. While, the patterns moving in dimension two were caused by the blend air temperature and hot air out damper, mechanical. When the pattern moves across the plot diagonally the variables of influence are a combination of process temperatures and mechanical.

\subsubsection{Projection to Latent Structures (PLS)}

The objective of model building using Projection to Latent Structures (PLS) is to determine the variables or combination of variables that influence outlet moisture content. These variables and the PLS-model may then be used to reduce the variation in outlet moisture content by understanding and controlling the variables. 
Projection to Latent Structures was conducted with the $\mathbf{X}$ and $\mathrm{Y}$ variables. The PLS model used the lagged variables as in the PCA. The result was four component/dimension model that utilized $81 \%$ of $\mathbf{X}\left(\mathrm{R}^{2} \mathrm{X}\right.$ of 0.806$)$ for explaining $95 \%$ of $\mathrm{Y}\left(\mathrm{R}^{2} \mathrm{Y}\right.$ of 0.952$)$ and cross validated prediction of $94.9 \%\left(\mathrm{Q}^{2}(\mathrm{cum})=0.949\right)$ of the response variation. Table 7.6 shows in more detail each component and the $R^{2} X, R^{2} Y$, and $\mathrm{Q}^{2}$ metrics. The first component explained $75.9 \%$ of the variation in the $\mathrm{Y}$ data, and predicted $75.7 \%$ of the variation in the $\mathrm{Y}$ data.

Table 7.6 PLS Model 1 [M5]

\begin{tabular}{ccccccc}
\hline Comp(A) & $\mathrm{R}^{2} \mathrm{X}$ & $\mathrm{R}^{2} \mathrm{X}(\mathrm{Cum})$ & $\mathrm{R}^{2} \mathrm{Y}$ & $\mathrm{R}^{2} \mathrm{Y}(\mathrm{Cum})$ & $\mathrm{Q}^{2}$ & $\mathrm{Q}^{2}(\mathrm{Cum})$ \\
\hline 1 & 0.396 & 0.396 & 0.759 & 0.759 & 0.757 & 0.757 \\
2 & 0.163 & 0.559 & 0.121 & 0.880 & 0.499 & 0.878 \\
3 & 0.155 & 0.714 & 0.051 & 0.931 & 0.416 & 0.929 \\
4 & 0.092 & 0.806 & 0.021 & 0.952 & 0.281 & 0.949 \\
\hline \hline
\end{tabular}

\subsubsection{Normality}

The residuals from the PLS model were first examined to determine if normality existed. The residuals appeared to be normally distributed when plotted in the normality plot. The residuals were then evaluated by treatment run. Figure 7.5, a time series plot of the residuals, shows that something was amiss as the second set of treatments specifically run \#5 (treatment - 42K 181F) had all positive residuals. The residuals for second set of the treatment combinations runs - \#5, \#6, and \#7 - were predominately positive residuals with the exception of the last run \#8 that were mostly negative. The model was under predicting the outlet moisture content for most of the second replicate of treatments. 


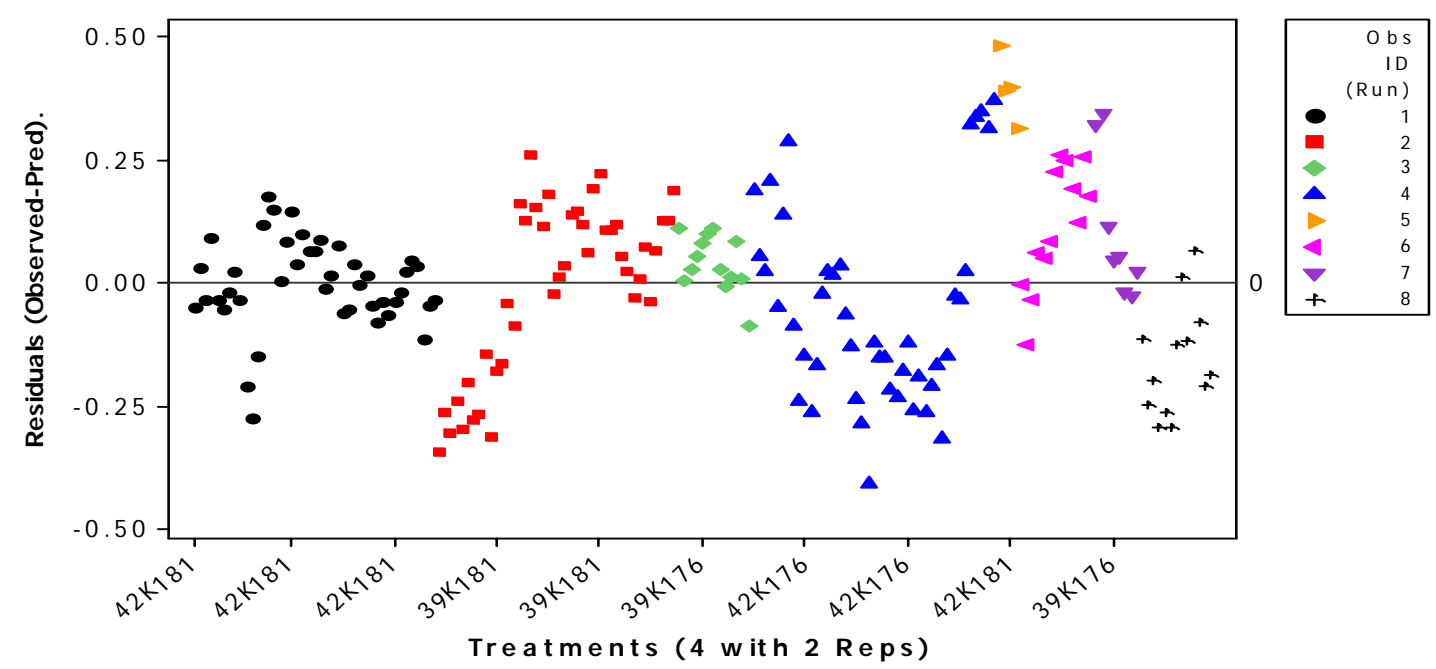

Figure 7.5 Residuals by Treatment and Run for PLS-Model 1

The score plot, Figure 7.6, was then analyzed looking at the 8 runs / classes to see if there were runs that were outliers. In general, there were no obvious outliers however runs 2 and 4 did have excursions in the lower left quadrant and in the upper right quadrant respectively.

The data set was then modified to determine what effect eliminating the second set or replicate of treatments would have on PCA-X. The PCA-X did not show much change as the number of components stayed at three and the $\mathrm{R}^{2} \mathrm{X}$ and $\mathrm{Q}^{2} \mathrm{X}$ had similar values as the first PCA model. In fact, neither the loading plot nor the score plot changed significantly. 


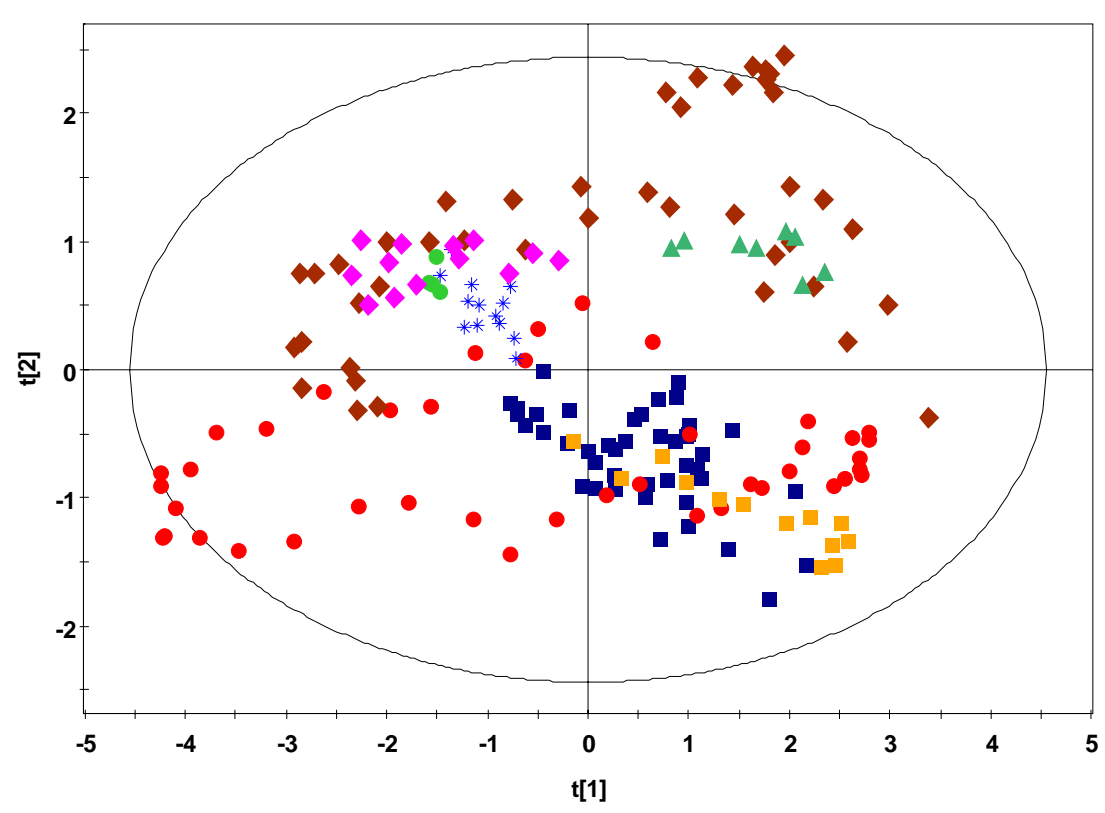

$\begin{array}{ll}* & \text { No Class } \\ * & \text { Class 1 } \\ * & \text { Class 3 } \\ - & \text { Class 4 } \\ - & \text { Class 5 } \\ - & \text { Class 7 } \\ - & \text { Class } 8\end{array}$

Figure 7.6 Score Plot for PLS Model with all Runs

The reduced data set was then modeled using PLS and the residuals were plotted as before in time series by treatment (Figure 7.7). There was improvement with all four treatments clustered around zero. Treatments \#2 and \#4 did have more scatter that the other two treatments. The normal probability plot showed the residuals were normal.

What happened to the second replicate? A review of the trial notes indicated at the end of the first replicate, the outlet temperature overshot the set point by more than 20 degrees. Dryer \#2, not being modeled in this research, started to swing the heat source with its demand for heat energy during the second replicate run. Adjustments were made, during the trial, but in hind sight and after reviewing the residuals, it apparently was not enough. Based on these findings, it was decided to continue to develop the model but remove the replicate set of data. 


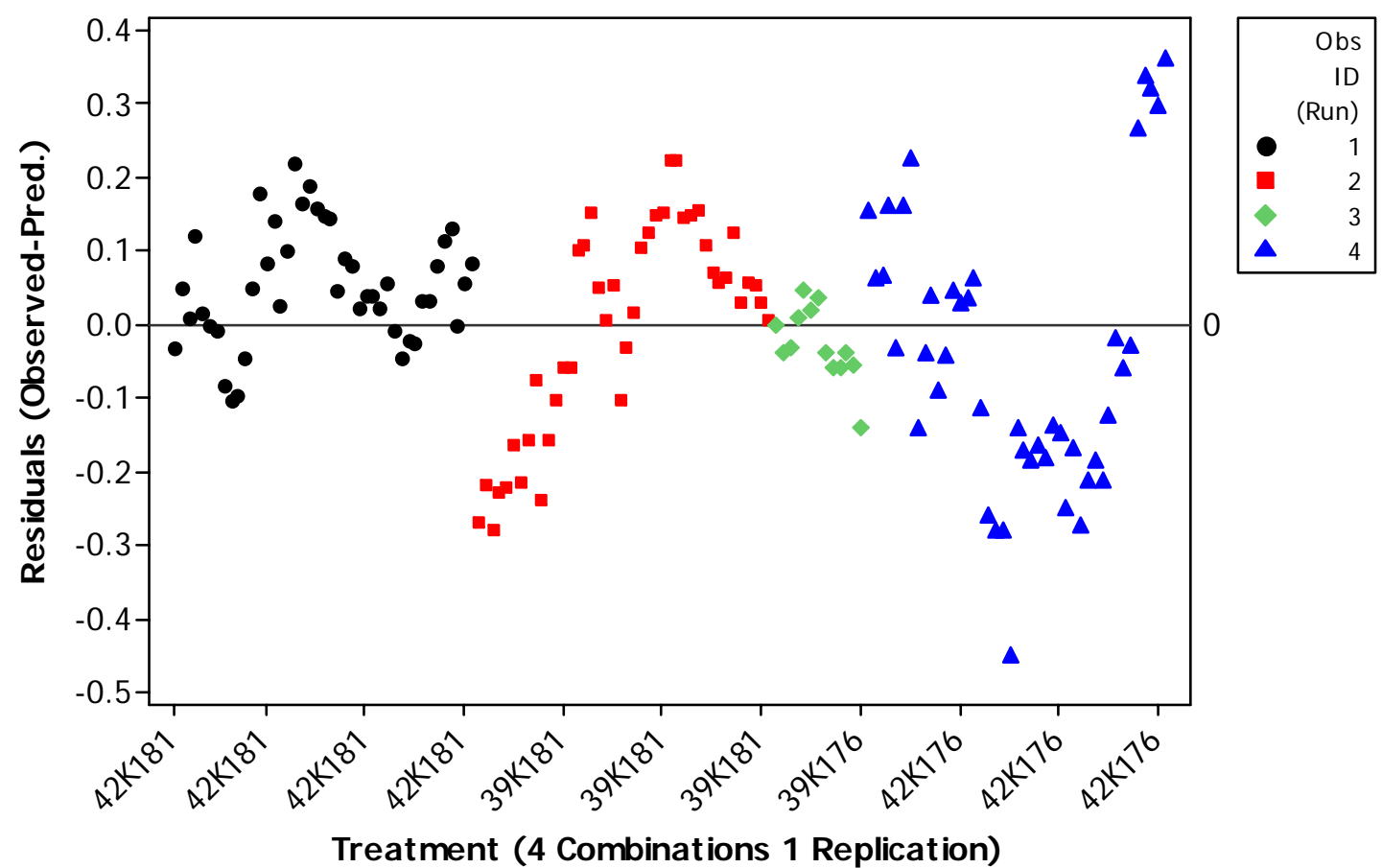

Figure 7.7 Residuals by Treatment and Run for PLS-Model 1 Replication

\subsubsection{PLS Model 2 with One Replication}

The PLS model with the first set of the treatment data, shown in Table 7.7, resulted in a model with four components that explained $95.2 \%\left(R^{2} Y\right.$ of 0.952$)$ and cross validated prediction of $94.9 . \%\left(\mathrm{Q}^{2}(\mathrm{cum})=0.949\right)$ of the response variation. Table 7.8 shows in more detail each component and the $\mathrm{R}^{2} \mathrm{X}, \mathrm{R}^{2} \mathrm{Y}$, and $\mathrm{Q}^{2}$ metrics.

Table 7.7 Treatments

\begin{tabular}{ccc}
\hline \hline Treatment & Feed Rate & Outlet Temperature Set Point \\
\hline 1 & 42,000 & 181 \\
2 & 39,000 & 181 \\
3 & 39,000 & 176 \\
4 & 42,000 & 176 \\
\hline
\end{tabular}


Table 7.8 PLS Model 2 [M6] One Replication of Treatments

\begin{tabular}{clccccc}
\hline \hline COMP(A) & $\mathrm{R}^{2} \mathrm{X}$ & $\mathrm{R}^{2} \mathrm{X}(\mathrm{CUM})$ & $\mathrm{R}^{2} \mathrm{Y}$ & $\mathrm{R}^{2} \mathrm{Y}(\mathrm{CUM})$ & $\mathrm{Q}^{2}$ & $\mathrm{Q}^{2}(\mathrm{CUM})$ \\
\hline 1 & 0.412 & 0.412 & 0.809 & 0.809 & 0.812 & 0.812 \\
2 & 0.213 & 0.625 & 0.074 & 0.883 & 0.385 & 0.881 \\
3 & 0.106 & 0.731 & 0.048 & 0.931 & 0.396 & 0.928 \\
4 & 0.121 & 0.852 & 0.021 & 0.952 & 0.29 & 0.949 \\
\hline \hline
\end{tabular}

\subsubsection{Observations - Score Plots}

The design of experiment factors, outlet temperature and feed rate, were considered important when developing the initial design and were expected to have an impact on the response variable, outlet moisture content. If they have an effect, then with multivariate data analysis, the score plot can be used to see the effect as each treatment's scores should be clustered together. If there is no effect, clustering will not be present but rather scattered and overlapped scores from the various treatments will be observed in the score plot.

Clustering is a qualitative visual test. The scores clustered together indicate they may be similar to one another and different from other scores further away. If the clustered scores are from the same treatment, then it probably means there is a difference in this treatment from the other treatments.

The score plot t[1] vs. t[2] in Figure 7.8 shows the scores for the four runs/treatments from the design of experiments. The plot shows some clustering for treatment/class 1 in the fourth quadrant with a little wandering toward the origin. Treatment 2 started as a cluster in the fourth quadrant but soon started to mosey about. Something changed in the process to drive it out of the cluster and all the way over to quadrant two. Treatment 3 shows a tight cluster of scores located in the second quadrant. 
Treatment 4 started as a very small cluster in the first quadrant and quickly started to ramble into the second quadrant and slightly into the third quadrant only to return to the first quadrant. Something in the process not being controlled by the experiment was changing and causing treatments \#2 and \#4 to leave the clusters.

The clustering seen in the score plot, Figure 7.8, indicates there may be an outlet temperature treatment effect on outlet moisture. This is visible in the second component, the vertical axis, by the separation of treatments \#3 and \#4 (both at 176F) from treatments $\# 1$ and \#2 (both at 181F). The feed rate effect on outlet moisture content would have been readily apparent had uncontrolled variation not occurred and caused treatments \#2 and \#4 to wander about. All four treatments started in their own quadrant, and while \#1 and \#3 stayed "put", \#2 and \#4 roved about. In the first dimension, treatments \#1 and \#4 (both at $42 \mathrm{~K}$ or 42,000 ) are predominately in the positive side; while treatment \#2 and \#3 (both at $39 \mathrm{~K}$ or 39,000$)$ are on the negative side.

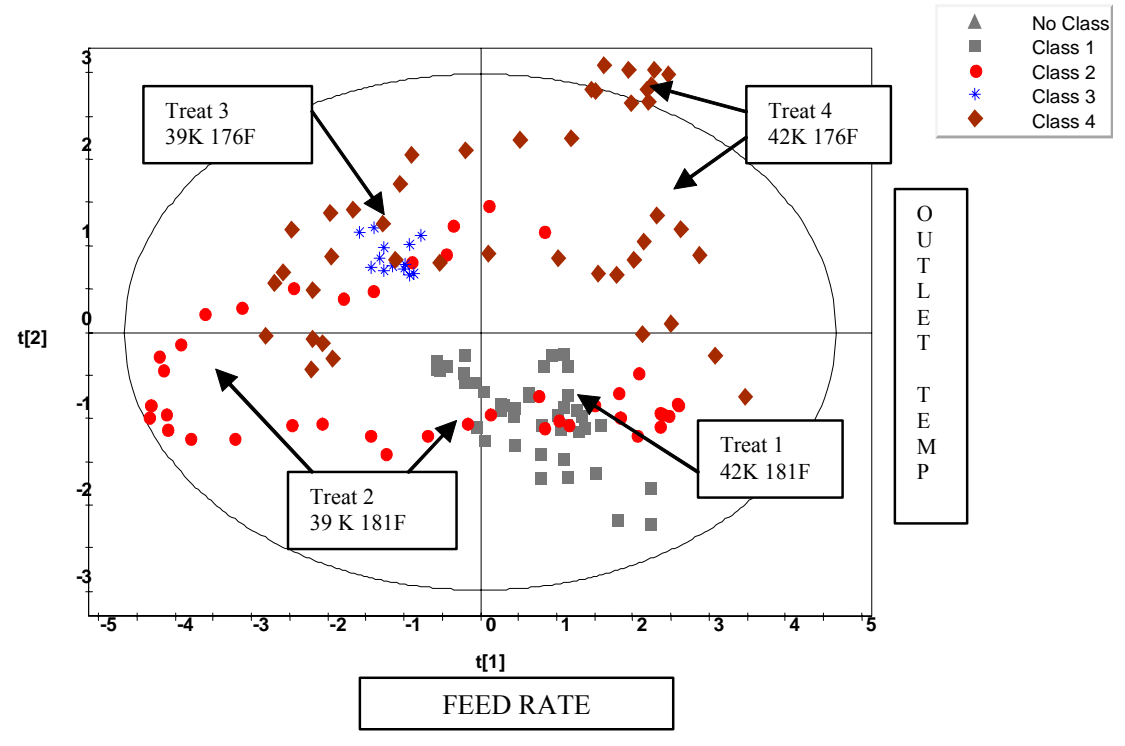

Figure 7.8 Score Plot $\mathrm{t}[1] / \mathrm{t}[2]$ 
The $t$ scores are created to correlate most highly with the response variable, $\mathrm{Y}$ as shown in the $\mathrm{t}[1]$ vs. $\mathrm{Y}$ [Outlet Moisture Content] plot (Figure 7.9). With this relationship, it is important to understand the $t$ scores and what variables influence them as these same variables then influence the response variable.

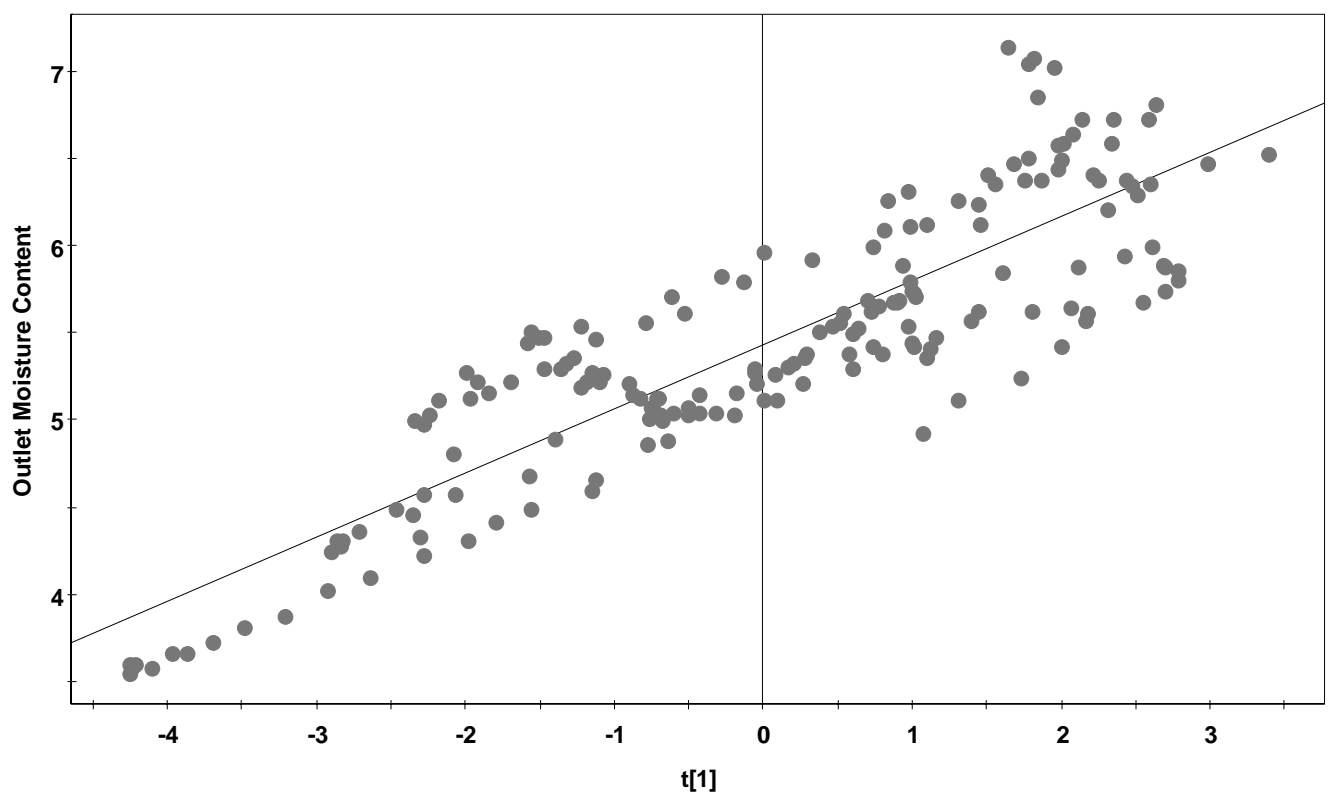

Figure 7.9 PLS-Model 2, t[1] vs. Y [Outlet Moisture Content]

The $t$ score plot for the first two components shows treatment \#2 moving through all four quadrants, beginning in the fourth quadrant but predominately wandering through the second and third. Treatment \#4 was also roving but stayed primarily in the first and second quadrants. To understand what may have caused the patterns, the scores are compared to the weighs for the variables. 


\subsubsection{Variables - Weight Plots}

The weight plots are used to study the scores plots to determine which variables were most influential in the trends or clusters seen in the scores plots. These same variables will in turn be influential with the outlet moisture content. Plotting the weights, $\mathrm{w}^{*} \mathrm{c}$, for the first two components facilitates finding which $\mathrm{X}$-variables were related to each other and Y. This is done by studying the clustering and scatter of the X's with each other and $\mathrm{Y}$.

The loading plot (Figure 7.10) shows that for the first component, depicted by the horizontal axis $\mathrm{w}^{*} \mathrm{c}[1]$, the response variable, outlet moisture content, is on the far right while the $\mathrm{X}$ variables with the exception of outlet pressure and the weight scale are on the opposite side of the zero $\mathrm{w}^{*} \mathrm{c}[1]$ vertical line. The positions indicate a positive correlation with respect to the first component between outlet moisture content, outlet pressure, and weight scale; while, outlet moisture content has a negative correlation with respect to the first component with the other $\mathrm{X}$ variables particularly the dryer temperatures, inlet and outlet temperatures, located the furthest away from outlet moisture content.

There is a strong correlation between outlet moisture content and outlet pressure because they are projected in the first component close together. The outlet pressure, which is a vacuum, typically increases or becomes more negative as more material is processed. When more material is processed, holding everything else constant, the outlet moisture content will raise thus a positive correlation. The correlation of weight scale with outlet moisture content indicates some positive correlation, but being low, 0.20 , indicates it is not as highly influential or highly correlated to the outlet moisture content as the outlet pressure. 


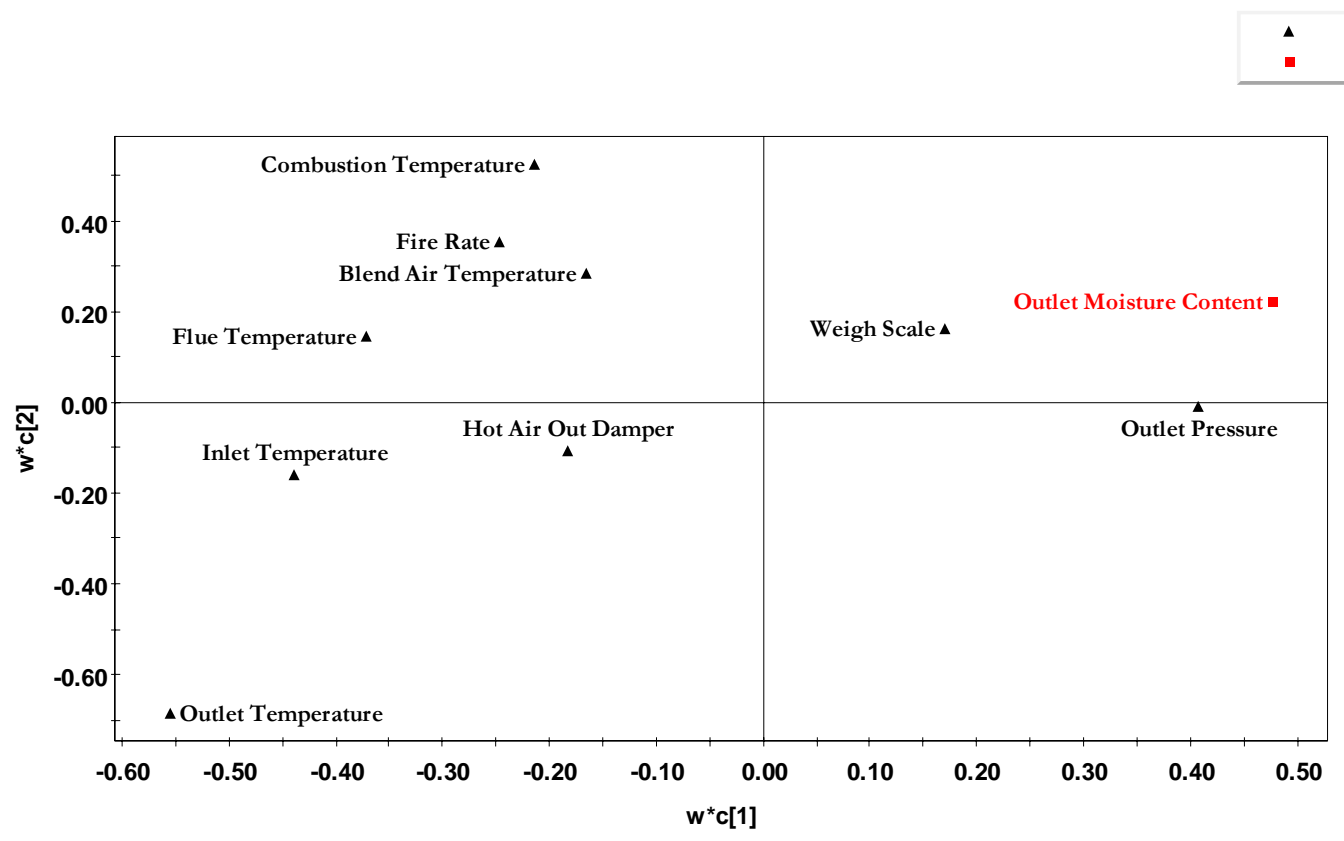

Figure 7.10 Loading Plot

The dryer temperatures are negatively correlated with outlet moisture content since they are on opposite sides in the first component, -0.55 to +0.47 . The spread between the variables is large indicating a strong negative correlation. Logically as the temperatures rise, the moisture is driven from the strand and moisture content falls. This relationship is what is expected. With this strong negative correlation, this first component might be referred to as the dryer component.

The second component, depicted by the vertical axis $\mathrm{w}^{*} \mathrm{c}[2]$ in Figure 7.10, shows the outlet temperature projected on the negative side of the second component; while, on the positive side are the heat source variables - combustion temperature, fire rate, and blend air temperature. All the other variables are close to the center line and are unimportant with respect to the second component as the weights are near zero. The arrangement indicates the dryer temperatures and the heat source are negatively 
correlated - as the outlet temperature goes up or down the heat source does just the opposite. This may signify that the heat source is not able to quickly respond to the outlet temperature demand for more heat energy. As the outlet temperature goes up, the heat source temperatures are pulled down. One might visualize the second component as the heat source component or the controls for the dryer temperature.

\subsubsection{Interpreting the Score Plot}

The weight plot and score plot were examined concurrently to comprehend the patterns seen in the score plot for treatments $\# 2$ and \#4. The plots can be studied as two components together or one component at a time. The discussion earlier about the wandering of the two treatments has them roving through two or three quadrants. By looking at one component at a time the mental picture may be simplified. First look for unusual patterns in the $t[1]$ scores, the horizontal axis. Then review the weight $\mathrm{w}^{*}[1]$ to ascertain the variable(s) with the greatest weight. The same is done later for the second component.

Figure 7.11, the score t[1] times series plot, shows scores cycling up and down with treatments $\# 2$ and $\# 4$ scores going from positive to negative. These were the two treatments already identified as wandering from their clusters in the score plot, Figure 7.8. What variables might be influencing these scores can be seen in the first component - horizontal axis for the loading plot, Figure 7.10, The outlet and inlet temperatures both have the large negative weights and they are positively correlated to each other. The outlet pressure has the third largest weight and is negatively correlated with the dryer temperatures. So as the dryer temperatures increase, the score decreases; and to further accentuate the score's downward trend, as the temperature increases the outlet pressure, a 
vacuum, drops becoming more positive. The first component was characterized as dryer in the discussion on variables and weight plots in section 7.2.3.2, and the score cycling reinforces the description. The original outlet temperature data collected during the treatment runs shows the cycling (Figure 7.11) seen with the scores. The original inlet temperature and outlet pressure also showed similar cycling (Figure 7.12).
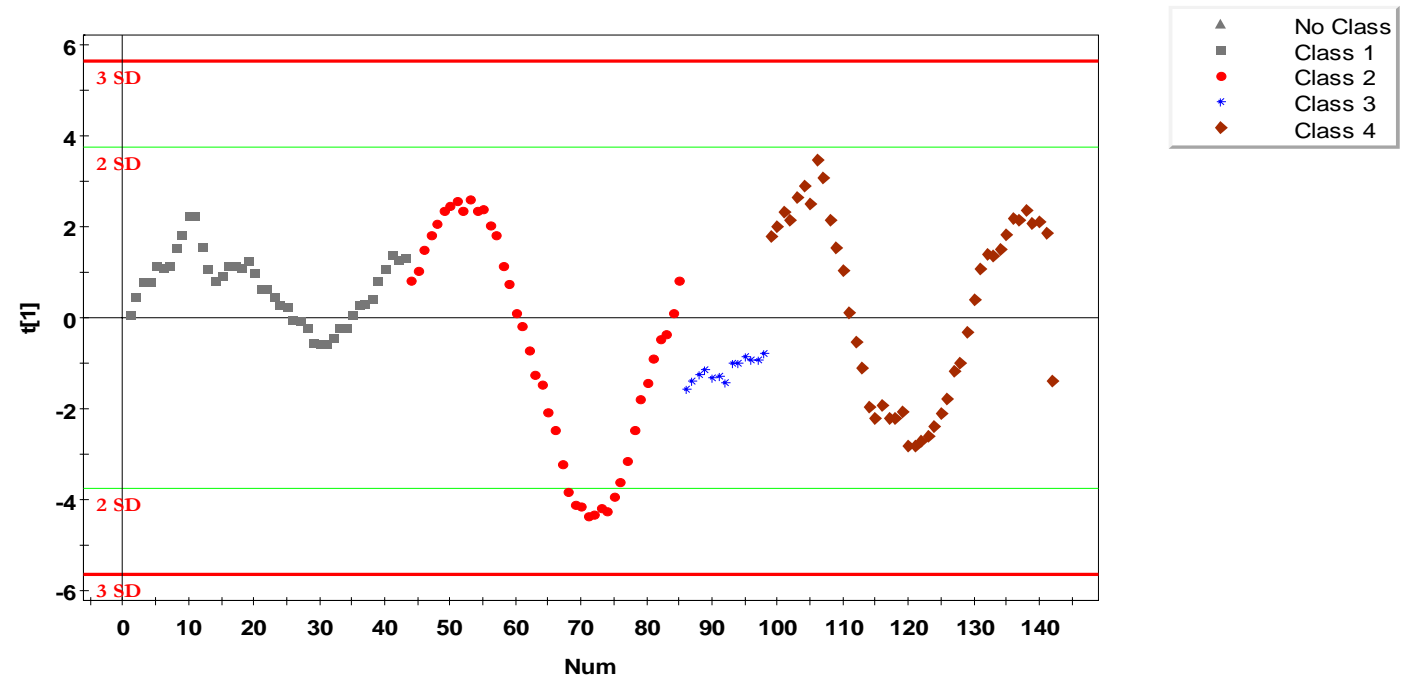

Figure 7.11 Times Series Plot of the Scores for PLS-Model 2 Component 1

Figure 7.13, the score t[2] times series plot, shows scores are negative for the first two treatments but positive for the last two treatments. Figure 7.10, the loading plot with respect to the second component, $\mathrm{w}^{*} \mathrm{c}[2]$, shows outlet temperature with a large negative weight, and the heat source variables, combustion temperature, blend air temperature, and flue temperature, with positive weights. Treatment $\# 1$ and $\# 2$ both had the DOE factor, outlet temperature, at the high level, $181^{\circ} \mathrm{F}$; while treatment $\# 3$ and $\# 4$ had the low level, $176^{\circ} \mathrm{F}$. The outlet temperature is associated with the t[2] scores as treatment $\# 1$ and $\# 2$ had high outlet temperature, and with a large negative weight these two treatment's 
scores would be lower than the other treatments with the lower outlet temperature factor level.

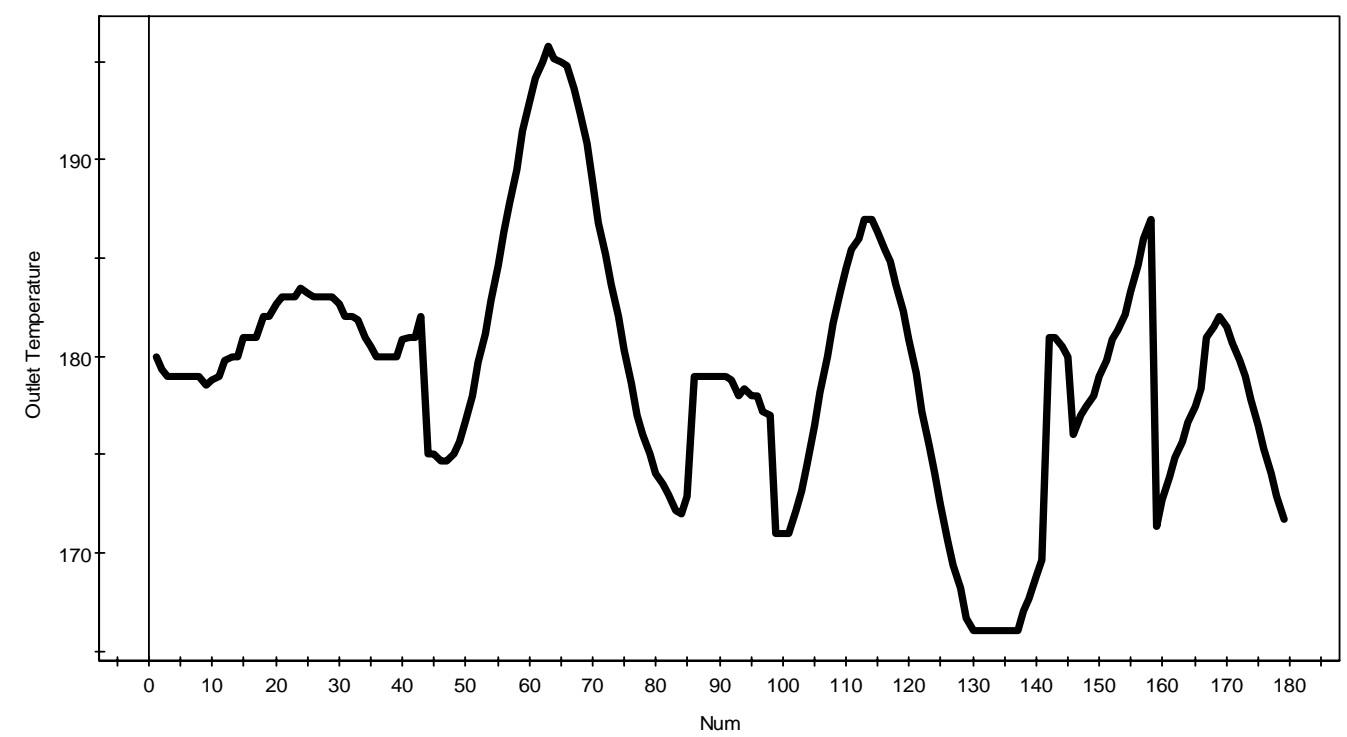

Figure 7.12 Time Series Plot of the Actual Outlet Temperature Data Collected
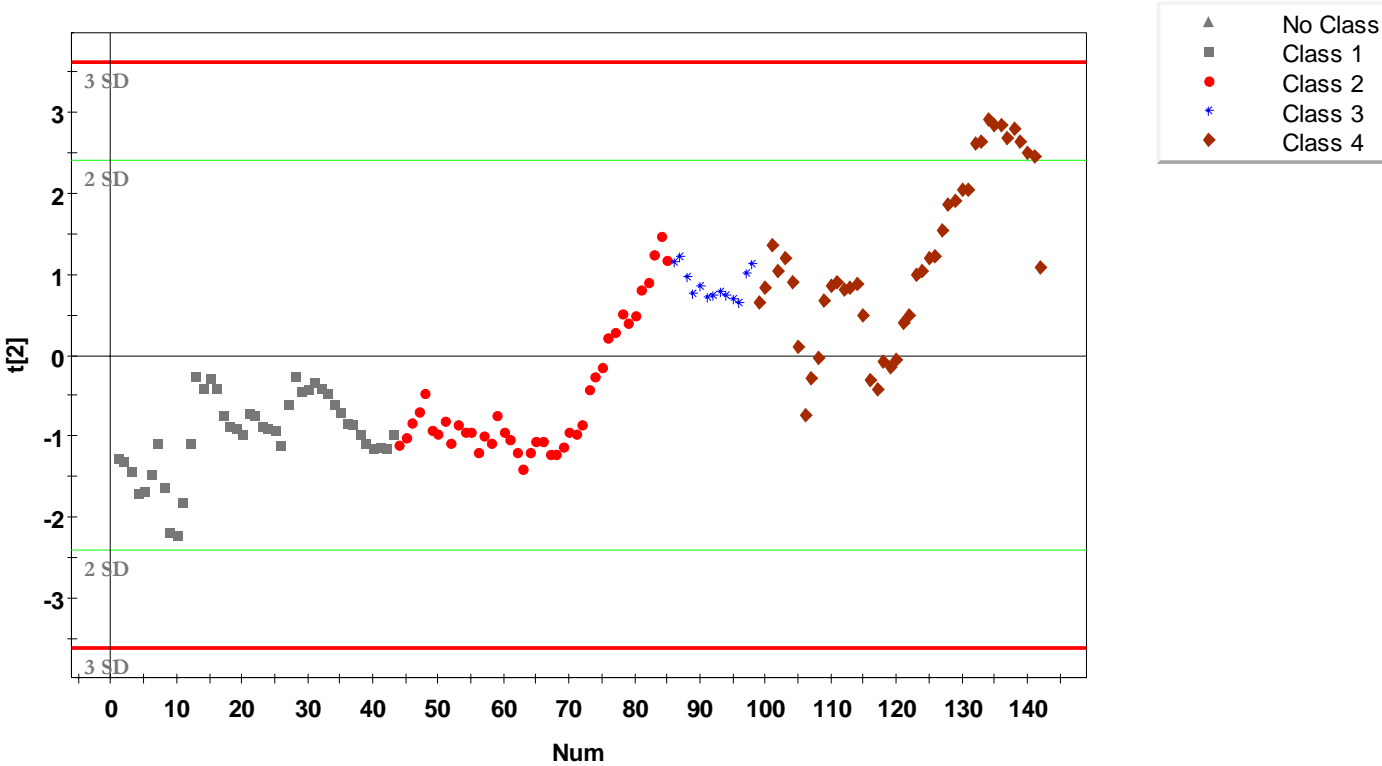

Figure 7.13 Times Series Plot of the Scores for PLS-Model 2 Component 2 
The loading plot with respect to the second component shows the outlet temperature negatively correlated with the heat source variables. This means when the outlet temperature is going down or up, the heat source temperatures are going just the opposite. This is referred to as heat source turndown, when the dryer does not need heat the heat source is slow to respond in this dimension. In other words, it takes a while to reduce the heat in the heat source or to build up heat energy. It can't be turned on or off in a "wink". This dimension was described as the heat source component or the controls for the dryer temperature. The second component had $\mathrm{R}^{2} \mathrm{Y}$ of only $7.3 \%$ and $\mathrm{Q}^{2}$ of $7 \%$. Component one, on the other hand, had $\mathrm{R}^{2} \mathrm{Y}$ of $81.4 \%$ and $\mathrm{Q}^{2}$ of $81.2 \%$. Component one or drying temperatures is the most influential component.

\subsubsection{Model Outliers}

The DModX and DModY are a measure of the part of the data the model does not capture in $\mathrm{X}$ and $\mathrm{Y}$ space respectively. It can be thought of as a plot of standard deviations of the residuals for each observation and is equivalent to the matrix row residual standard deviation. The DModX plot can be useful to see if there has been a "break in the correlations structure" of the data found by the model. The DModY plot can be used to see how well the response is explained by the model and if there are any departures.

Figure 7.14 shows there were two observations above the D-Crit line. The contribution plots of these points were studied along side the loading plot to see the break in the correlation structure. The break for the highest peak was the combustion temperature with the fire rate and blend air temperature. These three variables should be 
positively correlated instead at this point that was not the case as the combustion temperature was negatively correlated to the other two. The second highest peak's break in correlation structure was combustion temperature and outlet pressure. The two points were under three standard deviations so no observations at this point in the analysis were removed.

Figure 7.15 shows the DModY plot with a few spikes or departures. The values are the Y scaled residuals for that observation multiplied by the absolute value of the weight $u$ parameter. The value indicated a departure but at this point in the analysis no observations were removed as outliers.

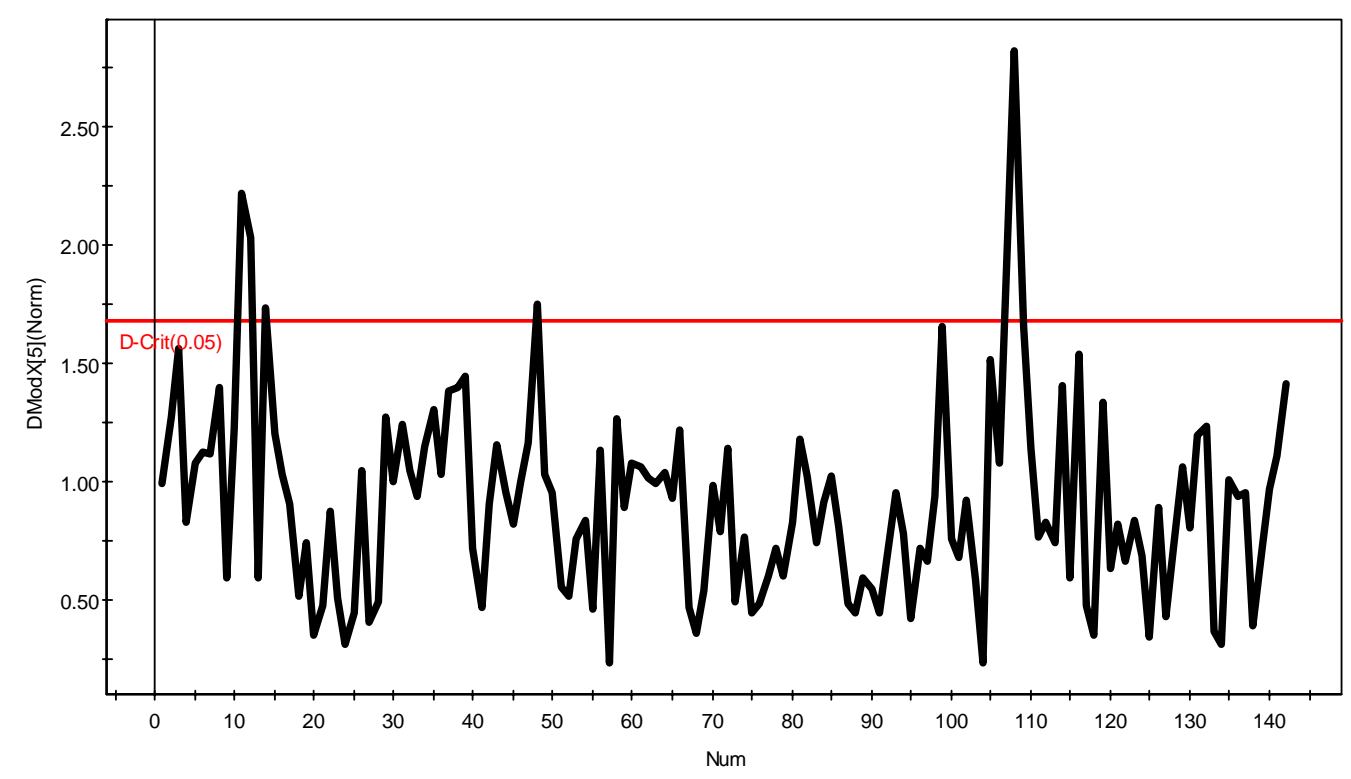

Figure 7.14 DModX 


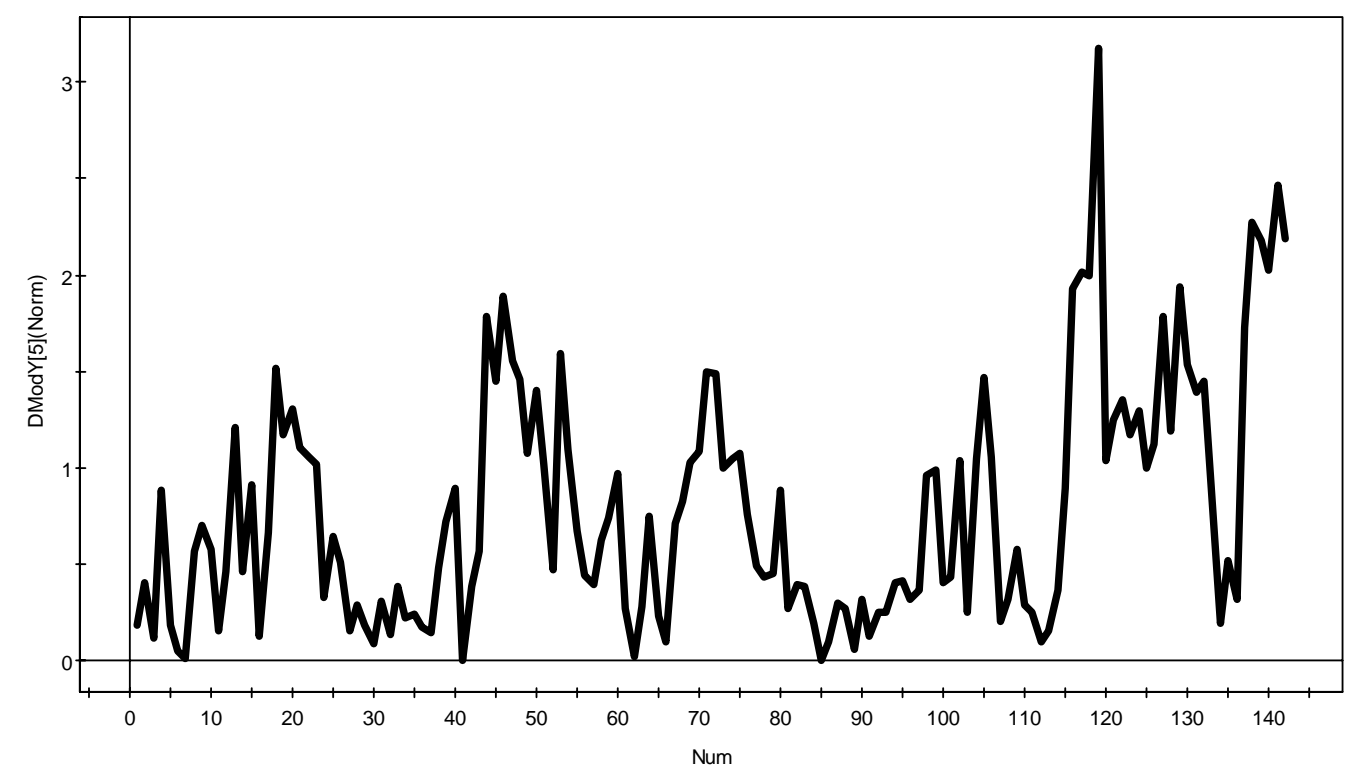

Figure 7.15 DModY

\subsubsection{Variable Diagnostics}

The Variable Influence on Projection (VIP) is a measure of the influence on Y of every variable in the model. VIP compactly summarizes the importance of the Xvariables. It is the sum of the squared PLS weights, $\mathrm{w}^{*}$ taking into account the amount of explained $\mathrm{Y}$-variance in each dimension. It is a measure of the relative magnitude of importance but does not tell if the influence is plus or minus since it a squared metric. A "normal" VIP value is 1.0. A value less than 0.6 indicate unimportant $\mathrm{X}$ 's. These variables can cause noise and reduce the $\mathrm{Q}^{2}$ so they should be removed.

The VIP plot, Figure 7.16, shows the weight scale variable with the lowest VIP value, below 0.60 , indicating low influence, and the possibility it was simply "noise" and adding little structure to the data. It was decided to remove each variable with a low VIP score, less than 0.60; but this was done one variable at a time. Since the variables are to 
some extent correlated, the model will change as they are removed; so removing one variable at a time, the effect on the model can be studied to see what if any affect occurs and if $\mathrm{Q}^{2}$ is improves.

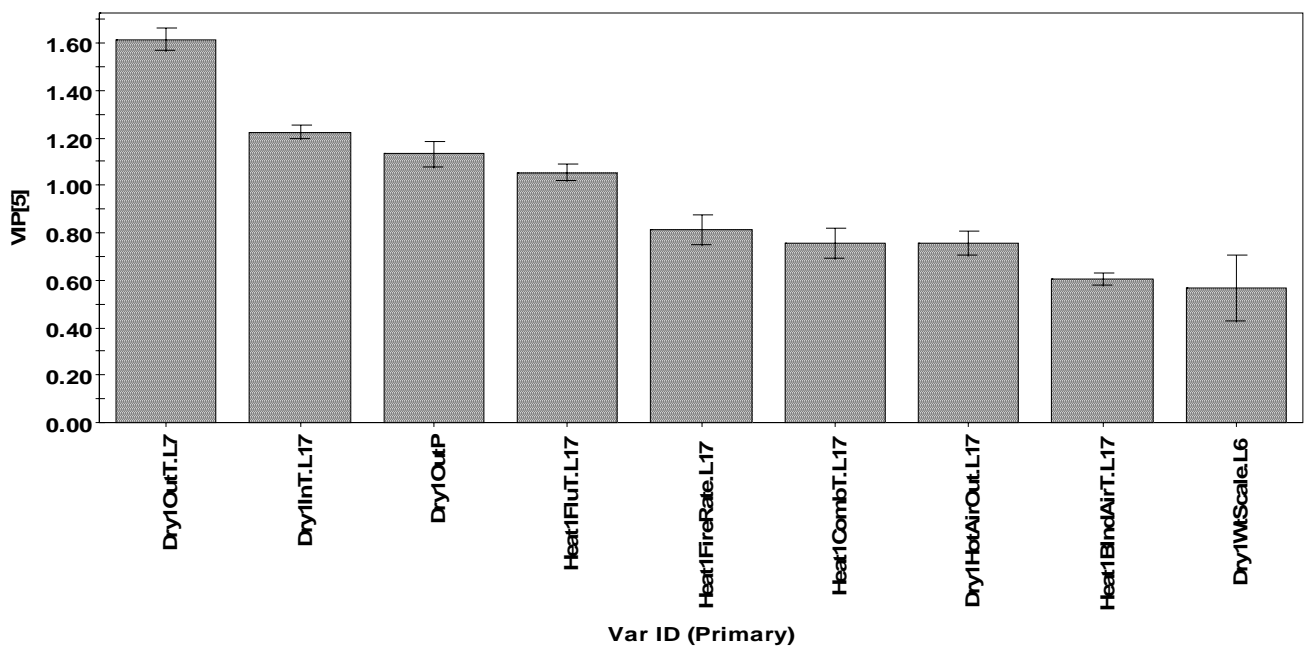

Figure 7.16 VIP Plot

\subsubsection{Final PLS Model}

The PLS model was re-run without the weight scale variable. The model was evaluated using plots of scores, loadings, DModX, DModY, and VIP. The VIP plot was utilized to remove additional variables with levels of influence of less than 0.60 . One variable at a time was removed, and the model re-run and evaluated for improvements in $\mathrm{Q}^{2}$. Four PLS models were run. In the end five variables remained - Outlet Temperature, Inlet Temperature, Combustion Temperature, Flue Temperature, and Blend Air Temperature. These were the variables with the most influence with VIP values over 0.60 .

The final model contained four statistically significant components. The first two components were the most important; while, last two components were minor adding 
0.051 to the $R^{2} Y$ (cum). Table 7.9 contains the information for each component's $R^{2} X$, $\mathrm{R}^{2} \mathrm{Y}$, and the $\mathrm{Q}^{2}$ metrics. A majority of the variation in the outlet moisture content is explained by the first two components; consequently the last two were dropped as they were not meaningful in explaining the variation even though they were statistically significant.

Table 7.9 Final Model 7 [M13]

\begin{tabular}{ccccccc}
\hline Components & $\mathrm{R}^{2} \mathrm{X}$ & $\mathrm{R}^{2} \mathrm{X}(\mathrm{cum})$ & $\mathrm{R}^{2} \mathrm{Y}$ & $\mathrm{R}^{2} \mathrm{Y}($ cum $)$ & $\mathrm{Q}^{2}$ & $\mathrm{Q}^{2}($ cum $)$ \\
\hline 1 & 0.599 & 0.599 & 0.842 & 0.842 & 0.840 & 0.84 \\
2 & 0.226 & 0.825 & 0.064 & 0.906 & 0.402 & 0.904 \\
3 & 0.087 & 0.912 & 0.033 & 0.939 & 0.346 & 0.937 \\
4 & 0.063 & 0.975 & 0.018 & 0.957 & 0.288 & 0.955 \\
\hline \hline
\end{tabular}

\subsubsection{Observation Outliers}

The score plots for the final PLS model were examined for potential outliers. There were no outliers in the first two components but there were several interesting trends and one cluster of data (Figure 7.17) that are discussed in section 7.2.4.4.
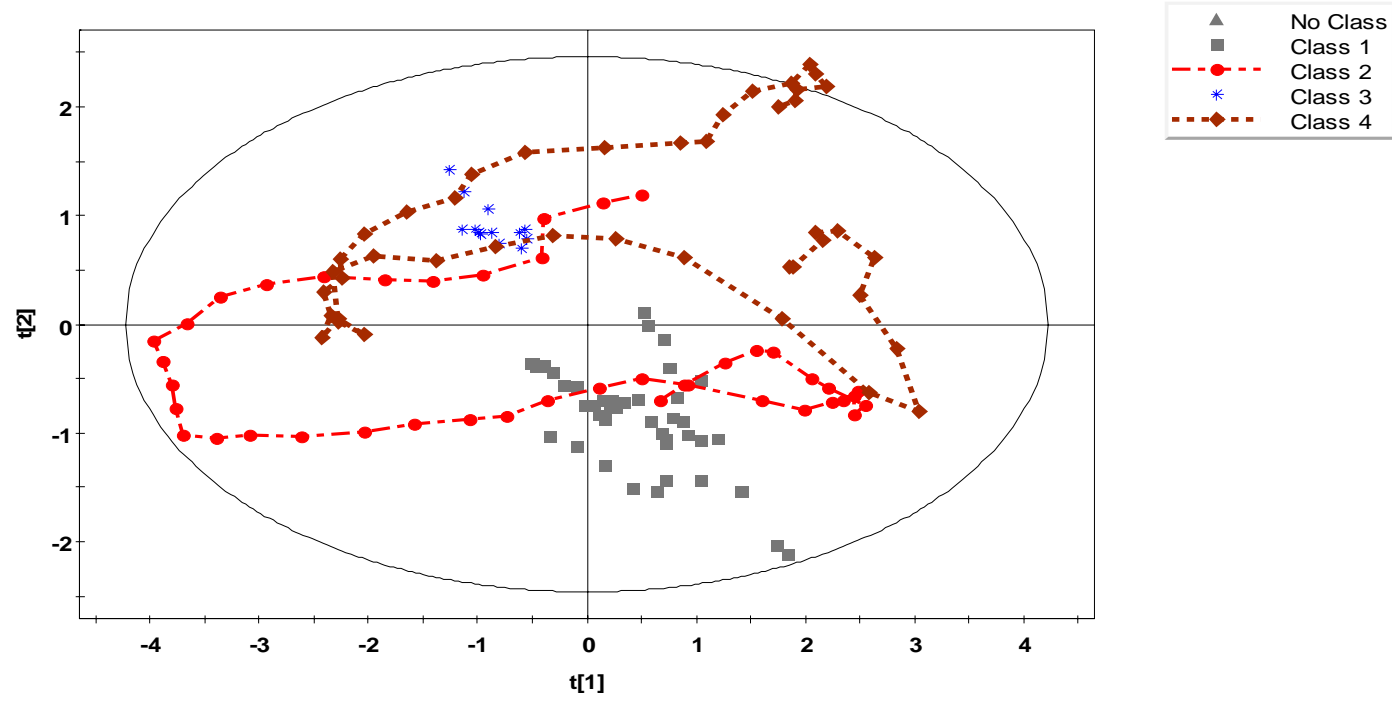

Figure 7.17 Score Plot t1/t2 Model 7 [M11] 


\subsubsection{Model Outliers}

The DModX (Figure 7.18) shows several residuals above the D-Crit line. The departures were less than three standard deviations - a cut off used by Eriksson et al. (2001) to indicate when more detailed evaluation is needed.

The departure at two time periods, two highest peaks, however may indicate issues with the model and a "borderline" ability to adequately capture these observations. The shift in the correlation structure for both was the combustion and flu temperatures.

The DModY (Figure 7.19) shows a departure at the end of the trial. This occurred when the DModY line peaked due to the outlet temperature dropping off significantly.

None of the "potential" outliers were removed.

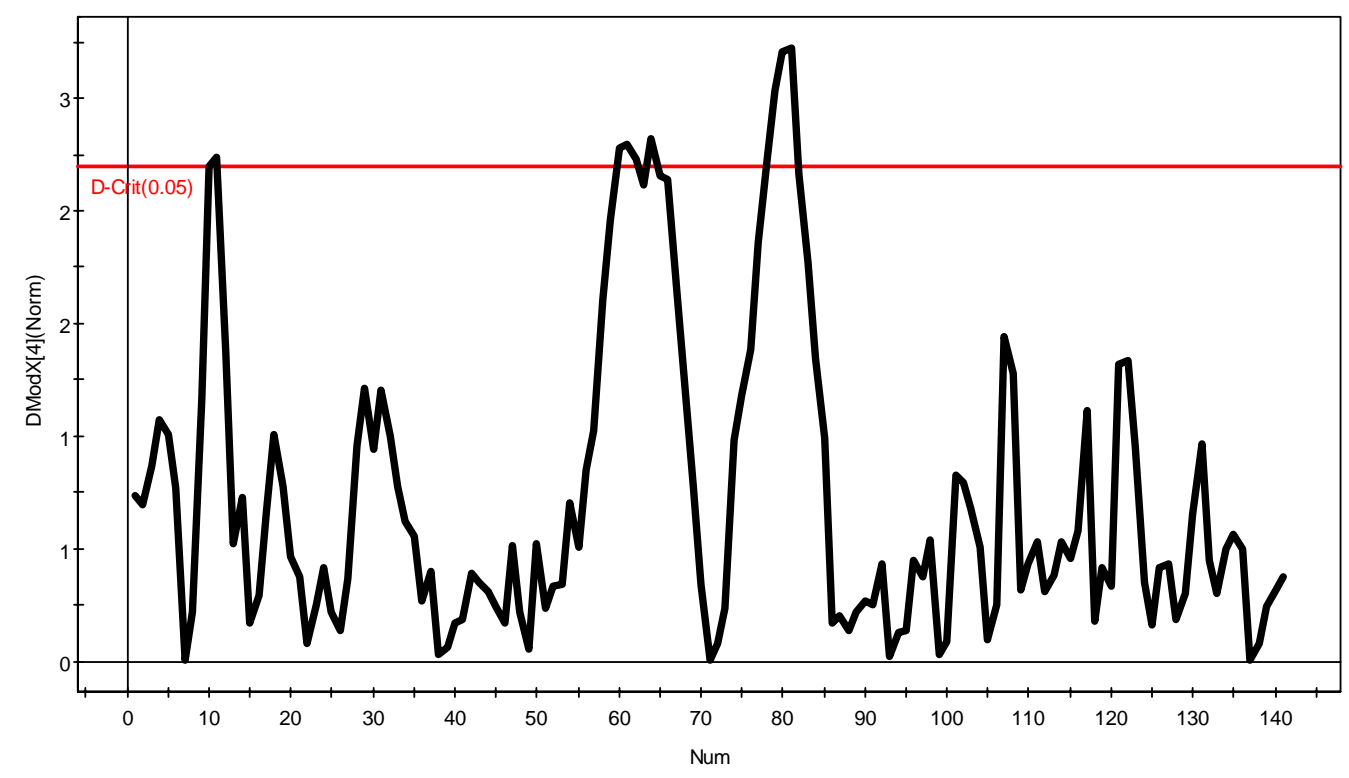

Figure 7.18 DModX plot for PLS-Model 7 [M11] 


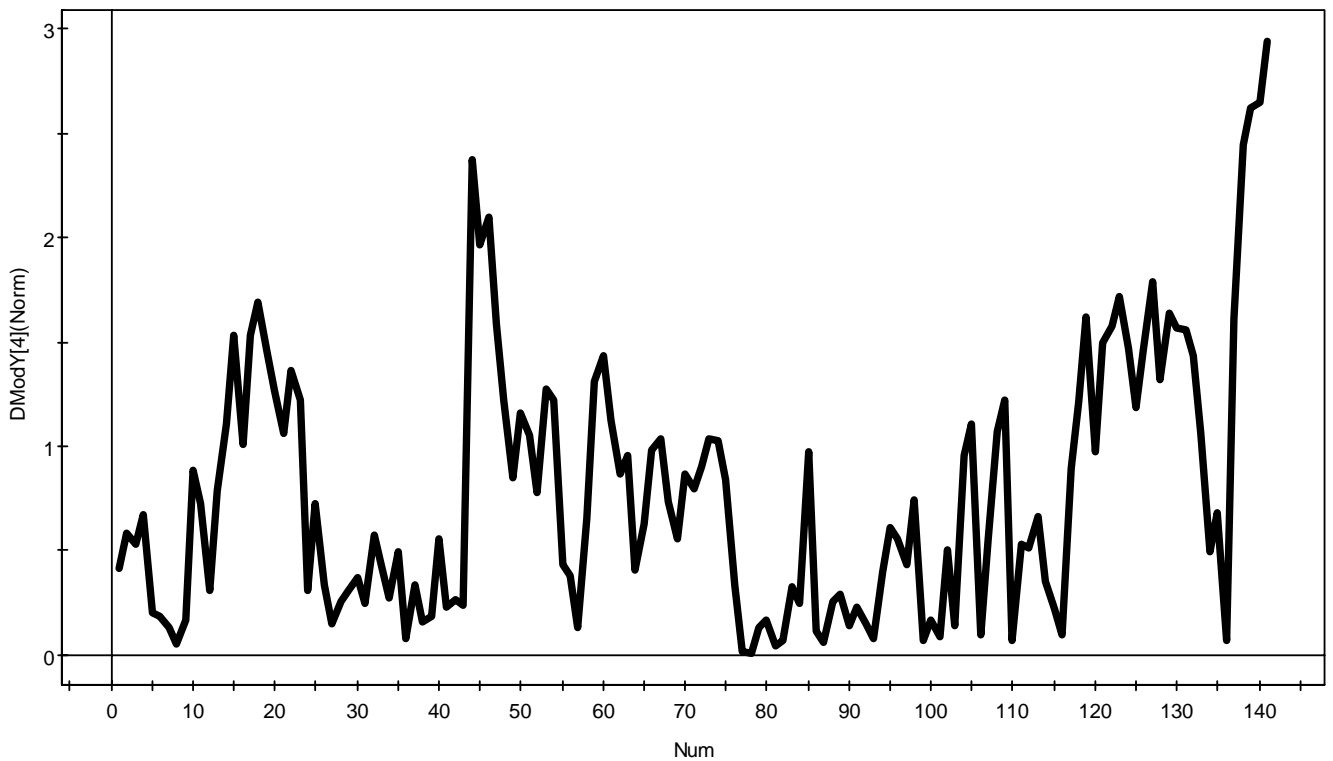

Figure 7.19 DModY plot PLS-Model 7 [M11]

\subsubsection{Variables Analysis - Loading Plots}

To understand the scores, the loading plots for the components have to be scrutinized, understood, and if possible characterized. The loading plots were examined to see the relationships among the predictor variables and with the response variable. The loading plot for the first and second component is shown in Figure 7.20. Table 7.10 lists the weights $\left(\mathrm{w}^{*}\right)$ for individual variables by component.

Table 7.10 Variable Weights by Component

\begin{tabular}{lcc} 
Variable & $\mathrm{w}^{*}[1]$ & $\mathrm{W}^{*}[2]$ \\
\hline Outlet Pressure & 0.440 & -0.009 \\
Inlet Temperature & -0.480 & -0.016 \\
Outlet Temperature & -0.600 & -0.730 \\
Combustion Temperature & -0.230 & 0.610 \\
Flue Temperature & -0.230 & 0.350 \\
\hline Component & Dryer & Heat Source \\
Characterization & & Turn Down \\
\hline
\end{tabular}




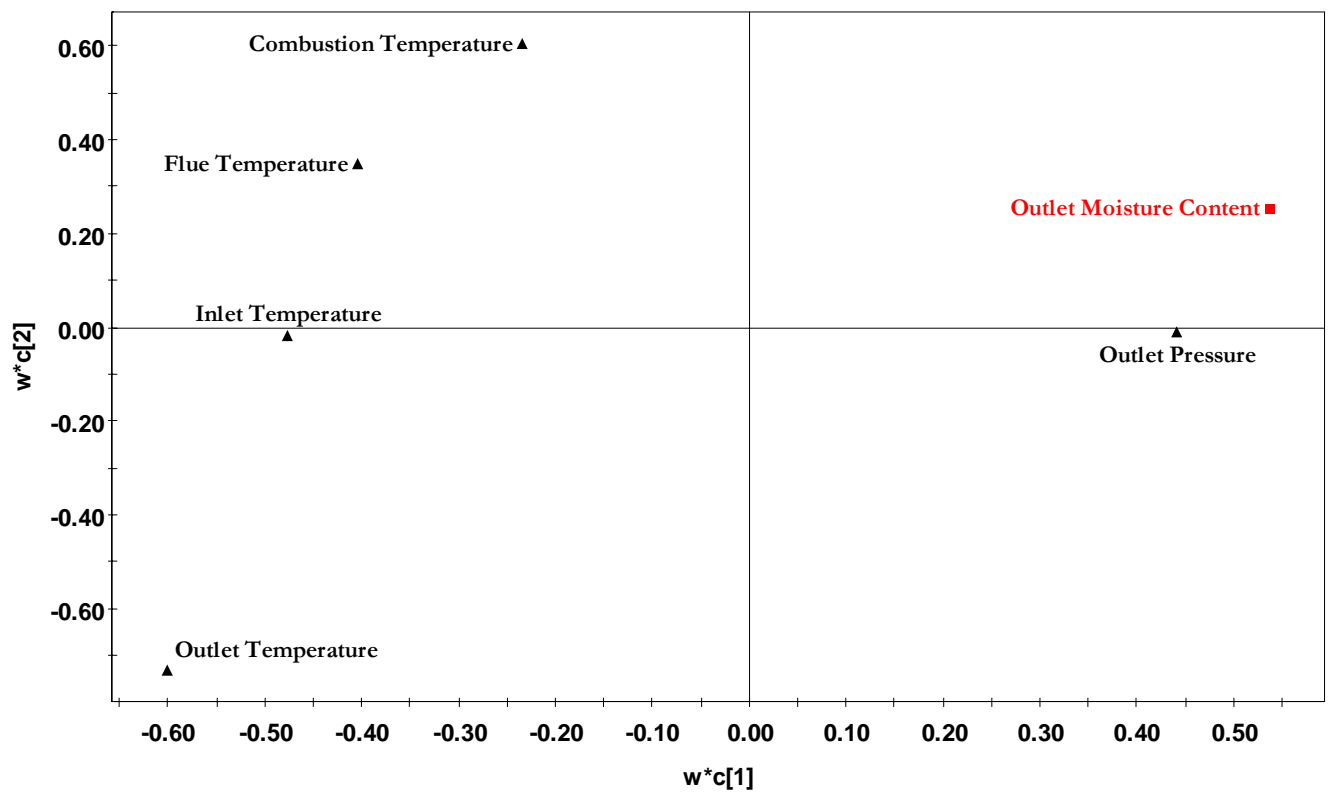

Figure 7.20 Loading Plot for Model 7 [M13]

The first component shows a large weight, $\mathrm{w}^{*}[1]$, for outlet temperature and reasonably large weights for the inlet temperature and outlet pressure. The dryer temperatures are both projected to the same quadrant in the loading plot, making them positively correlated. They are projected to the diagonally opposite quadrant to the quadrant the outlet $\mathrm{MC}$ is projected to; thus, making them negatively correlated with outlet MC. As dryer temperatures increase, the outlet MC's decrease and vise versa. The outlet pressure, on the other hand, is projected to the same side as the outlet moisture content indicating positively correlation. The outlet pressure typically increases, becomes more negative, with an increase in the feed rate; and as more furnish initially enters the dryer, the outlet moisture content increases.

The first component can be characterized as the dryer component as it is a combination of inlet and outlet temperatures and outlet pressures. A contrast or linear 
combination of the variables for this component might be outlet pressure - \{outlet + inlet temperature . The first component had a $\mathrm{R}^{2} \mathrm{Y}$ of $84.2 \%$ and a $\mathrm{Q}^{2}$ of $84.0 \%$.

The second component indicates that as the outlet temperature decreases, the heat source temperatures increase and vise versa. The second component could be characterized as heat source turn down with a contrast of $\{$ combustion + flue temperatures\} - outlet temperature. The second component added an additional $6.4 \%$ to the $R^{2} Y$ value.

\subsubsection{Observation Analysis - Interpreting the Score Plot}

The scores plot for the first and second component shows several trends and one data cluster (Figure 7.21). The first trend begins at the beginning of the trial with treatment \#1. Observations 1 to 12 are in the third and fourth quadrants and are moving left to right almost horizontally across the first component - indicating the change is primarily in the first component with some in the second component. The $t[1]$ scores are increasing and the $\mathrm{t}[2]$ scores are decreasing.

To determine which variables are influencing the trend, the loading plot (Figure 7.20) is used since both loading and score plots can be superimposed. The major contributor to this trend is the combustion temperature located diagonally opposite where the observations are trending. The weights for combustion temperature are negative with respect to the first component and positive with respect to the second component. The scores and weights would indicate the combustion temperature is dropping. The dropping combustion temperature is shown in Figure 7.22. Figure 7.23 shows t[1] scores are increasing while the $\mathrm{t}[2]$ scores are decreasing. 

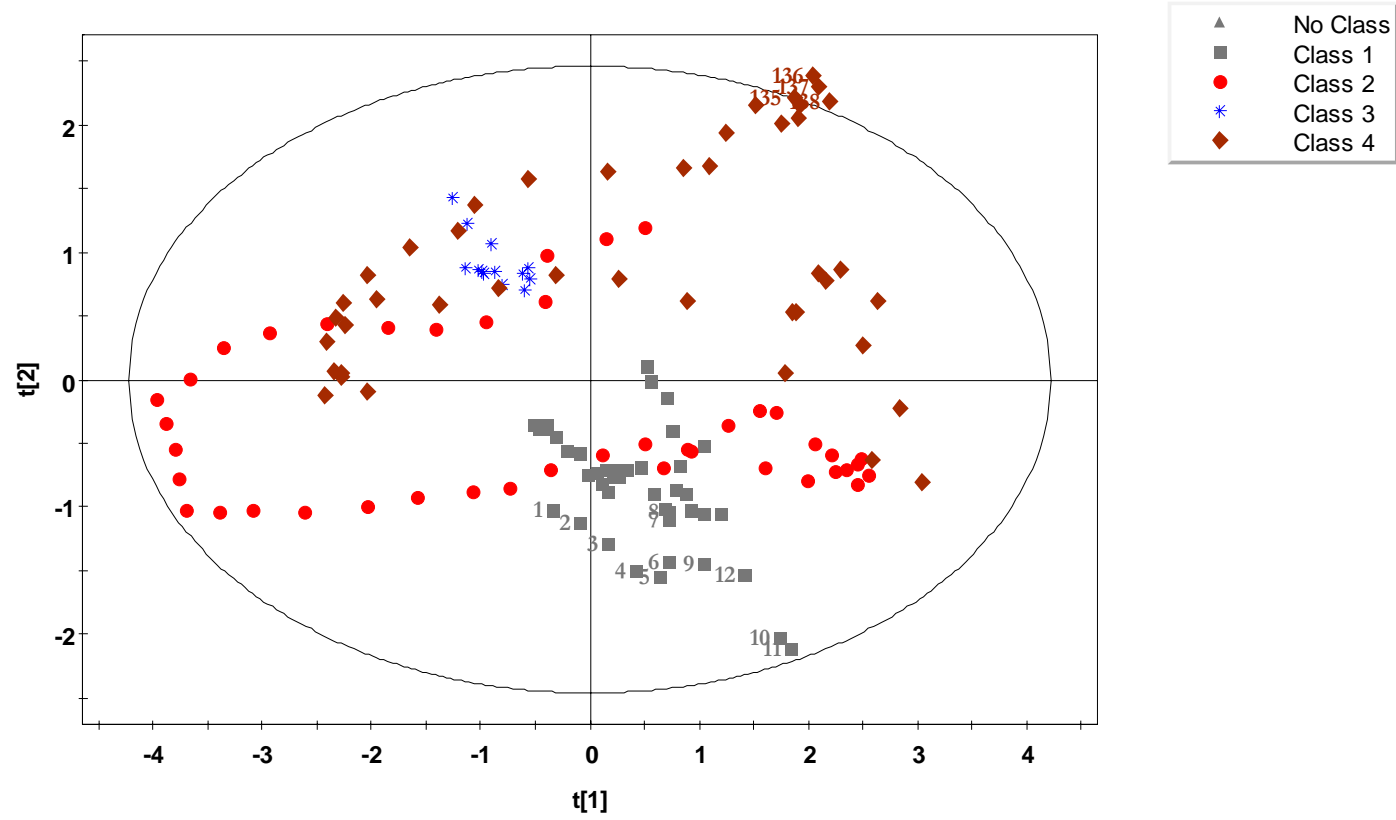

Figure 7.21 Score Plot with Treatment \#1 Pattern 1 to 12 in the Fourth Quadrant

The contribution plot is useful in examining scores, and the plot (Figure 7.24) showed the combustion temperature as the major contributor.

A cluster of data, observations 135 to 138 , is located in the first quadrant on the upper edge of the ellipse. The major contributor is outlet temperature. As the scores increased in this cluster, the outlet temperature was actually decreasing. In fact the raw data for the outlet temperature showed it had actually backed way off and was over 10 degrees below target. This is shown in the time series plot for outlet temperature (Figure 7.25), and the contribution plot indicates outlet temperature was the largest contributor (Figure 7.26). 


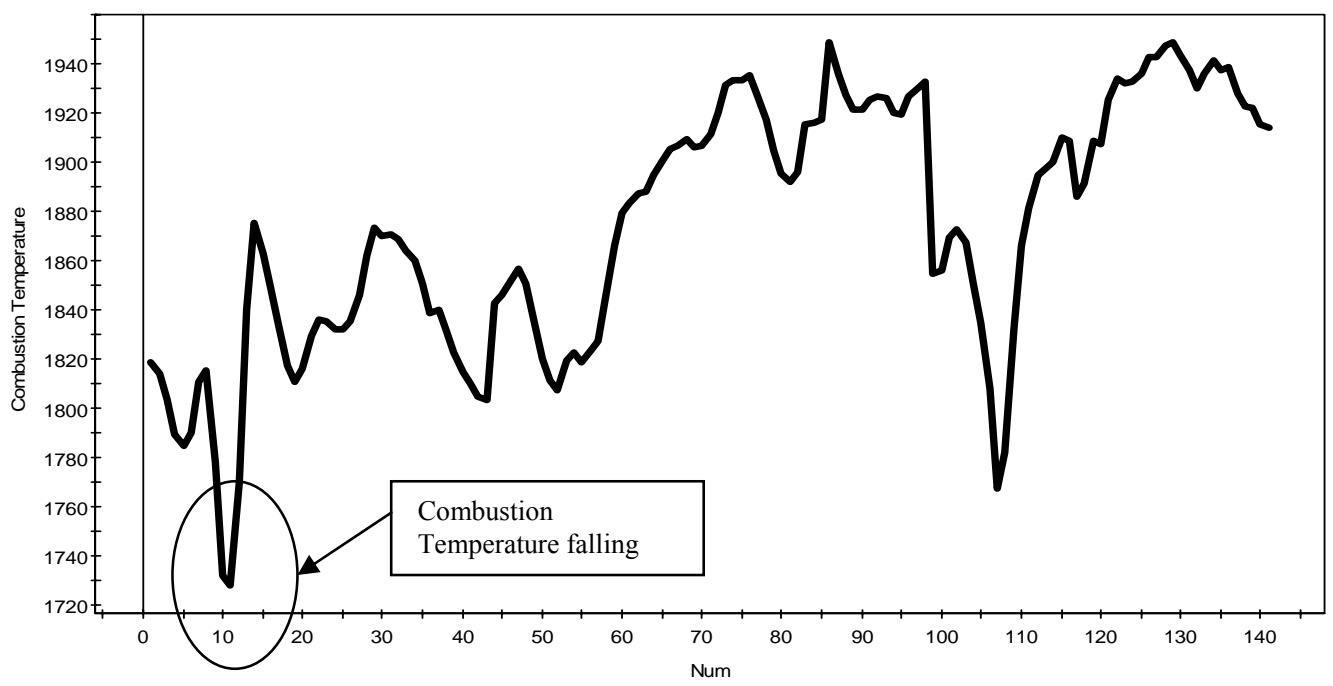

Figure 7.22 Combustion Temperature Time Series Plot

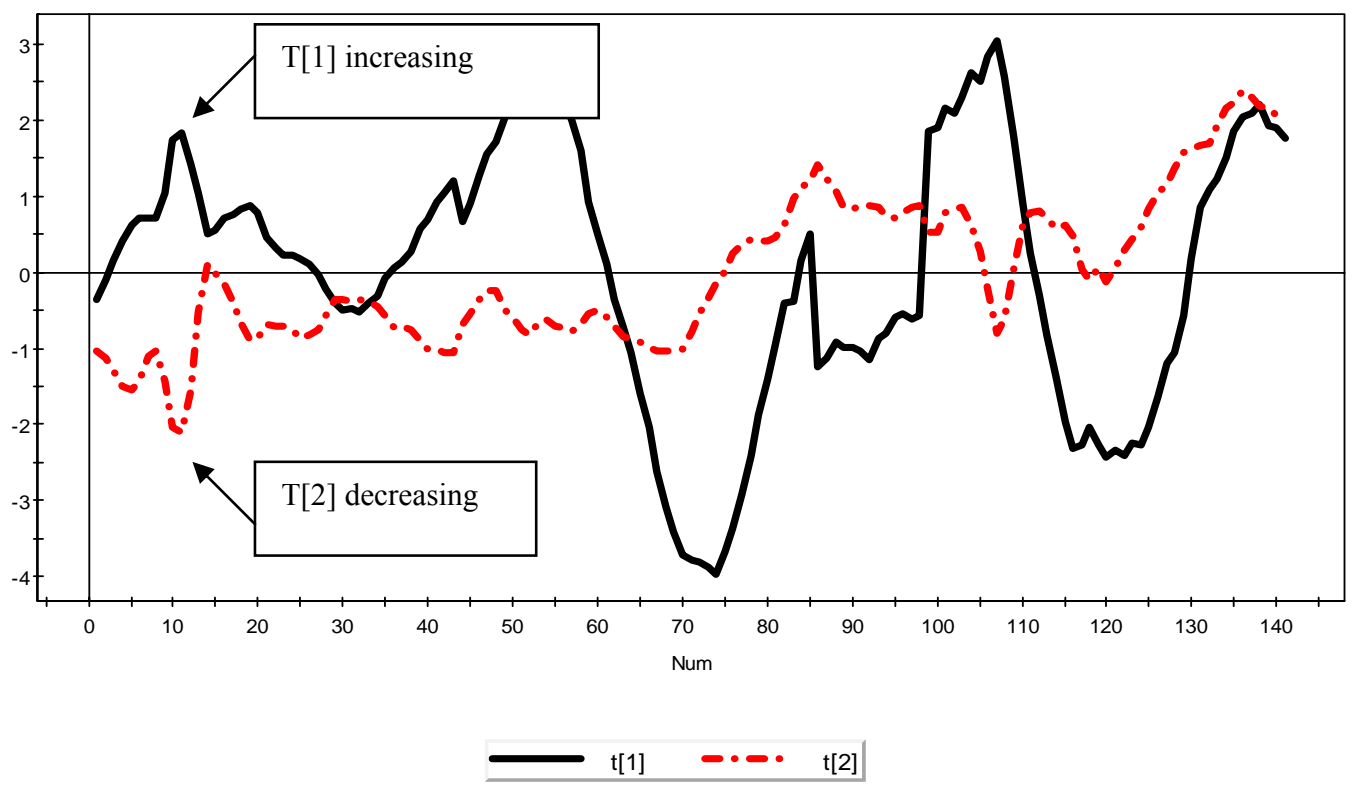

Figure $7.23 \mathrm{t}[1]$ and $\mathrm{t}[2]$ Time Series Plot 


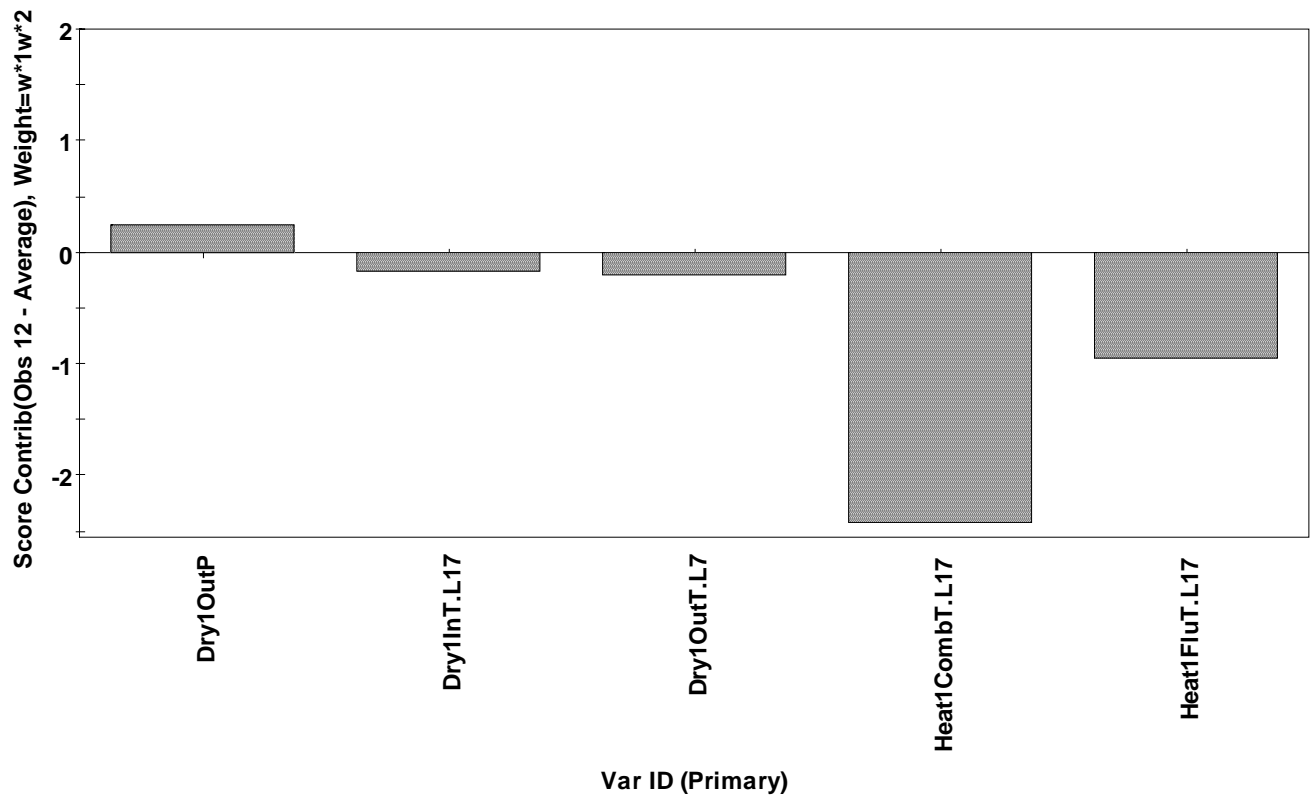

Figure 7.24 Contribution Plot for Observation 11

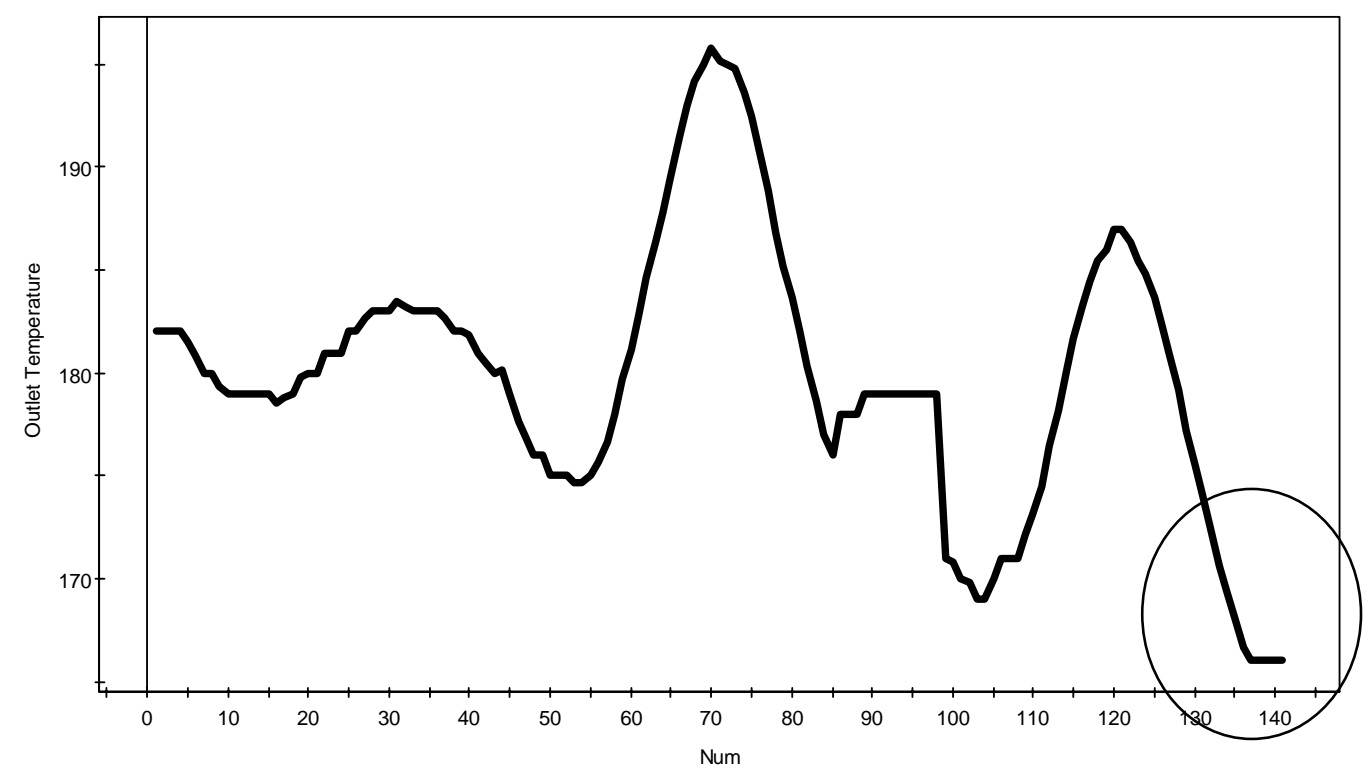

Figure 7.25 Outlet Temperature Time Series Plot - Circle Shows Drop in Temperature. 


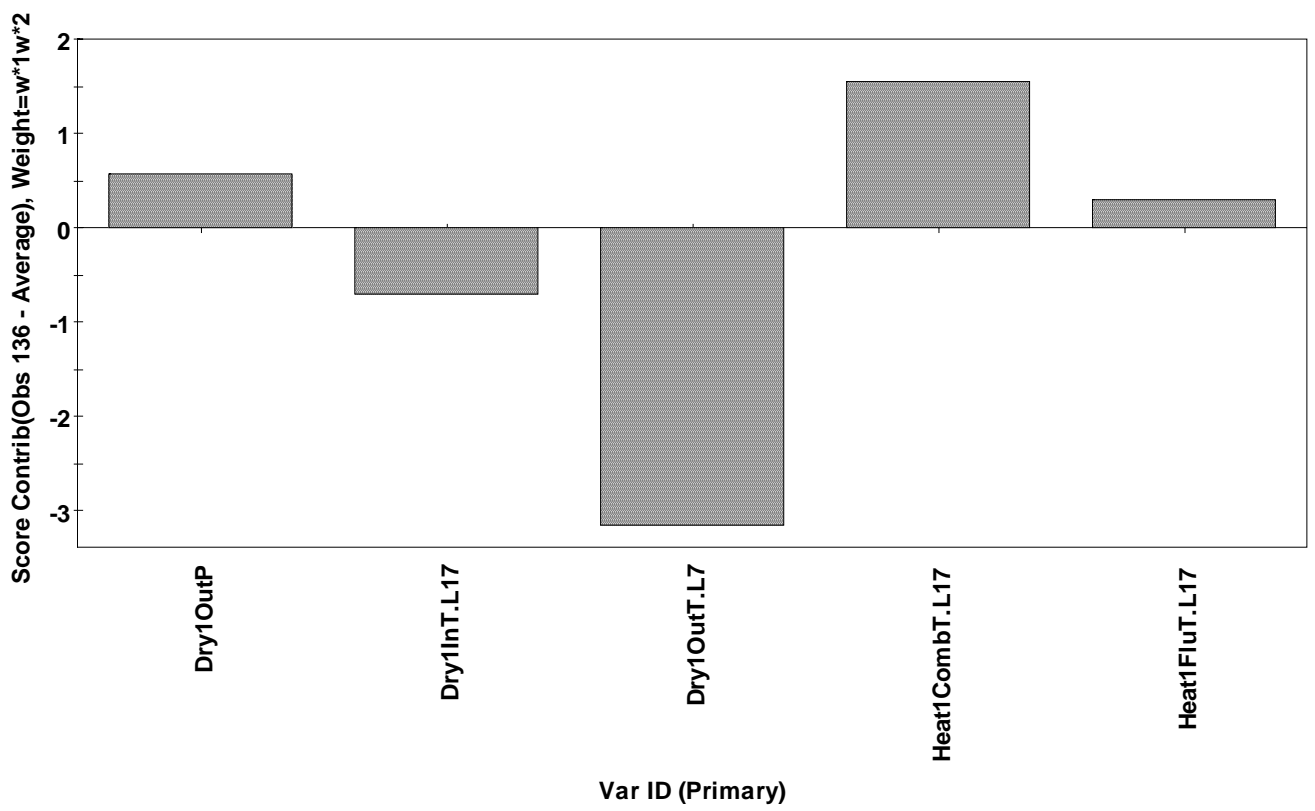

Figure 7.26 Contribution Plot for Observation 135

\subsubsection{Variable Diagnosis}

Understanding the variables was discussed in section 7.2.4.3 by examining the loading plots. A visual technique for analyzing variables is with the Variable Influence on Projection (VIP) plot. It helps to visual the relative magnitude of importance of the various variables as shown in Figure 7.27. Again outlet temperature is first, followed by inlet temperature, and then outlet pressure. 


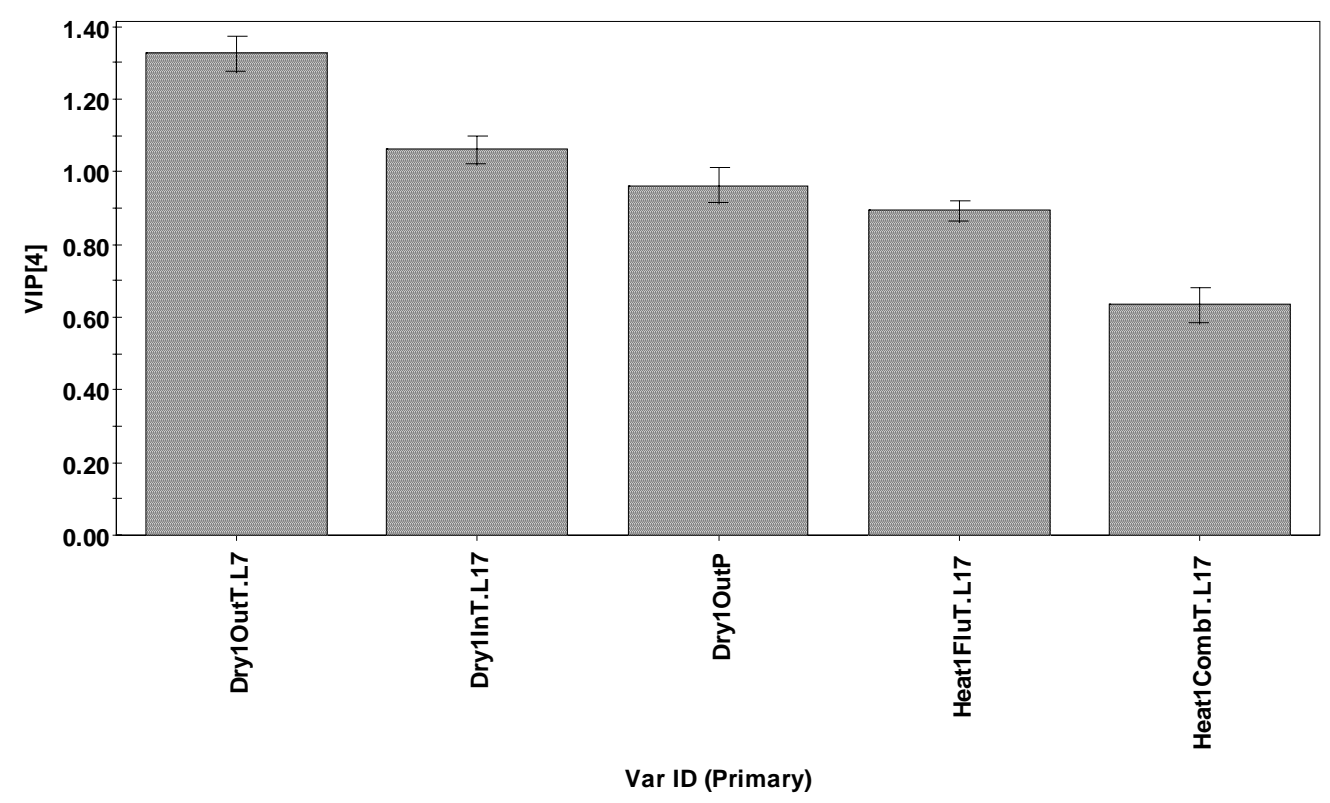

Figure 7.27 Variable Influence on Projection (VIP)

\subsubsection{Coefficients}

The model coefficients summarize the relationship of the $\mathbf{X}$ with $\mathrm{Y}$ across all dimensions (components). The coefficients are presented in Table 7.11 as both centered and scaled (CS) and raw coefficients. Centering and scaling the variables remove the magnitude and takes the scale out of the picture. The coefficients become dimensionless, and the relationship between variables easier to understand.

Table 7.11 Model Coefficients [M13] - Centered and Scaled as well as Raw for PLS

\begin{tabular}{lcc}
\hline \hline \multicolumn{1}{c}{ Variable } & Coefficient (CS) & Coefficient (Raw) \\
\hline Constant & 6.73396 & 26.4237 \\
Outlet Pressure & 0.06622 & 0.21757 \\
Inlet Temperature & 0.16631 & 0.002314 \\
Outlet Temperature & -0.916039 & -0.11051 \\
Combustion Temperature & -0.039414 & -0.000604 \\
Flue Temperature & -0.249105 & -0.002385 \\
\hline \hline
\end{tabular}


A visual presentation, Figure 7.28, of the coefficients (CS - centered and scaled) reinforces how influential outlet temperature is to outlet moisture content.

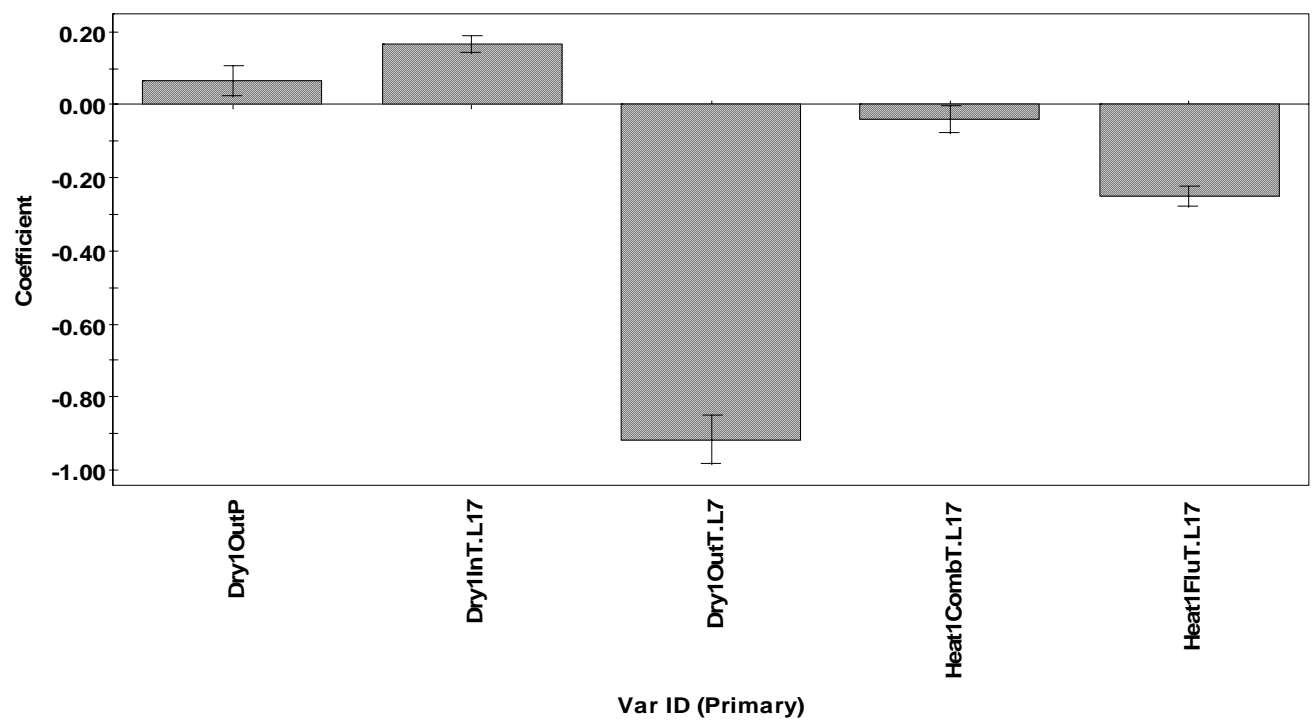

Figure 7.28 Coefficients (CS) for PLS Model 7 [M13]

The model equation using the raw numbers is:

Outlet Moisture Content $=26.4237+0.21757$ Outlet Pressure

+ 0.00231Inlet Temperature - 0.1105Outlet Temperature

- 0.00060Combustion Temperature - 0.00239Flue Temperature. (6-1)

\subsubsection{Validation}

SIMCA-P has a procedure called validate to estimate the significance of the estimated predicted power $\mathrm{Q}^{2}$. The procedure develops a number of parallel PLS models using randomly re-ordered $\mathrm{Y}$-data and evaluates both $\mathrm{R}^{2}$ and $\mathrm{Q}^{2}$. The order of $\mathrm{Y}$ is 
randomly permuted a number of times ( 20 by default) and separate models are fitted to all the permuted Y's extracting as many components as was done with the original $\mathrm{Y}$. SIMCA displays a validate plot (Figure 7.29) in which the $\mathrm{Y}$-axis represents the $\mathrm{R}^{2} \mathrm{Y}$ and $\mathrm{Q}^{2}$-values of all the PLS-models, including the real one; while the $\mathrm{X}$-axis shows the correlation coefficients between the permuted and original response variables. The plot has two regression lines one is fitted to $R^{2} Y$ and the other to $Q^{2}$. The intercepts are interpretable as measures of "background" $\mathrm{R}^{2} \mathrm{Y}$ and $\mathrm{Q}^{2}$ obtained by fitting random data. Experience shows that the $\mathrm{R}^{2} \mathrm{Y}$ intercept should not exceed $0.3-0.4$ and that the $\mathrm{Q}^{2}$ should not exceed 0.05. Intercepts below these indicate valid models. (Eriksson et al. 2001)

The validate procedure was run on the PLS model with 20 permutations and the results are promising (Figure 7.29). The original $\mathrm{R}^{2} \mathrm{Y}$ and $\mathrm{Q}^{2}$ values were higher than the corresponding "permuted" values. Both $\mathrm{R}^{2} \mathrm{Y}$ and $\mathrm{Q}^{2}$ intercepts were below the recommended cut off so a valid model was indicated. 
Modeling Data 2_27_04.M11 (PLS): Validate Model Dry1OutMC.L6 Intercepts: $R 2=(0.0,-0.00717), Q 2=(0.0,-0.146)$

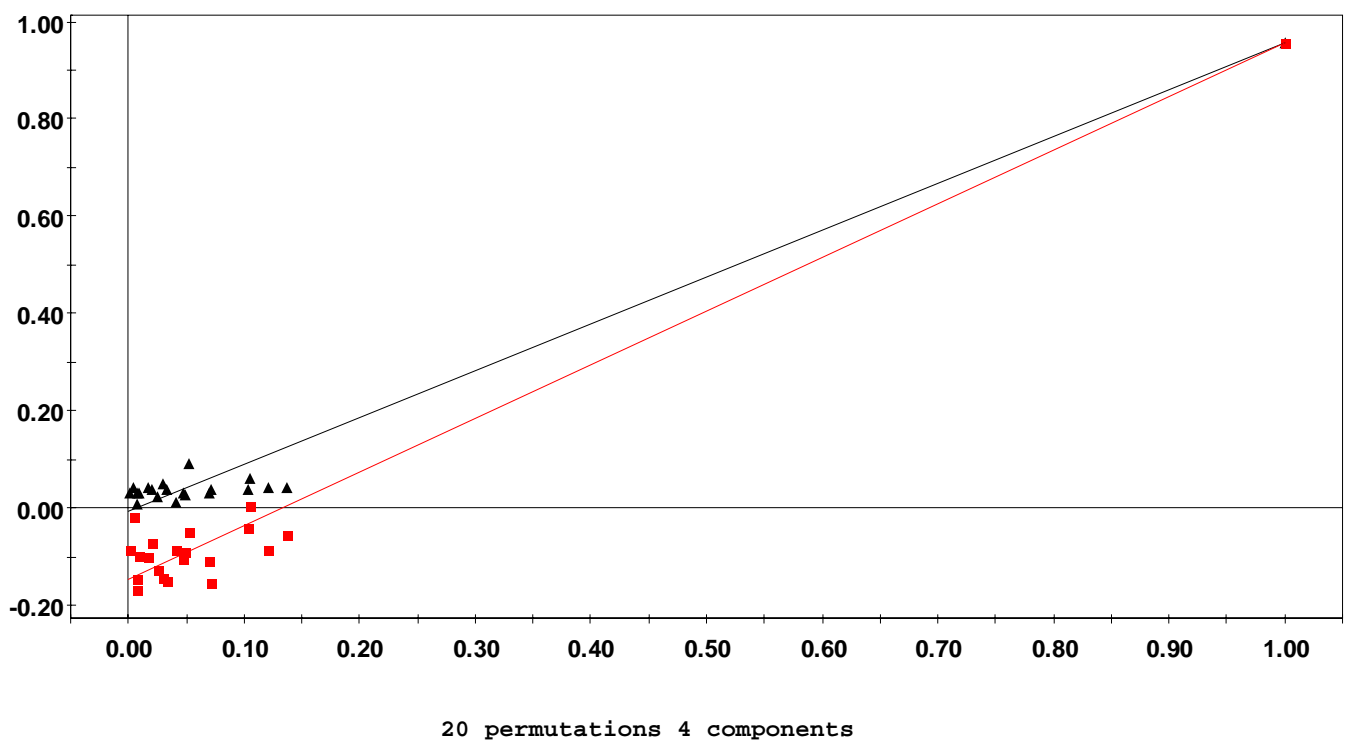

Figure 7.29 Validate Model with 20 permutations

\subsubsection{Summary of PLS Model Development}

The PLS Model for explaining and predicting outlet moisture content is two components or dimensions, consisting of five variables. The model explains $82.5 \%$ of the variation in the five variables and $90.6 \%$ in the outlet moisture content. It also predicts $90.4 \%$ of the outlet moisture content variation. The first component is related to the dryer and accounts for over $85 \%$ of the explained and predicted variation in the outlet moisture content. The second component is characterized as heat source turn down. It includes the heat source along with the outlet temperature and explains an additional $6.4 \%$ of the variation in the outlet moisture content. 


\subsection{Model Validation}

Model Validation using "external" data, i.e. data not previously used is considered the best method to determine the predictive ability of a model. To confirm the validity of the PLS model, data were exported from the IP21 data historian and used to predict outlet moisture content. All the data sets were pre-processed - lagged, mean centered and unit scaled. The data sets were imported into SIMCA-P and the PLS-Model was used to predict the outlet moisture contents.

\subsubsection{Prediction Set 1}

The first external data set or prediction data set was collected from the process approximately 12 hours after the original data were collected for the design of experiment.

The prediction scatter plot is shown in Figure 7.30. The root mean square error of prediction (RMSEP) is the standard deviation of the predicted residuals and was 0.378 for the first prediction data set. The RMSEP can be used to calculate the $95 \%$ confidence interval for the predicted outlet moisture content which would be $\pm 0.76 \%$.

The model predicted moisture content well on the low end. The model predicted $5.4 \%$ and the observed was $5.5 \%$. On the upper end the model slightly under predicted. The coefficient of determination, $\mathrm{R}^{2}$, was 0.9379 , indicating the regression equation explained about $94 \%$ of the variation in the observed moisture contents.

The predicted scores were reviewed to determine if the reason the predictions were slightly off could be explained by the scores. For the first two components - dryers and heat source turn down - the scores were in the center of the ellipse with no outliers (Figure 7.31). 


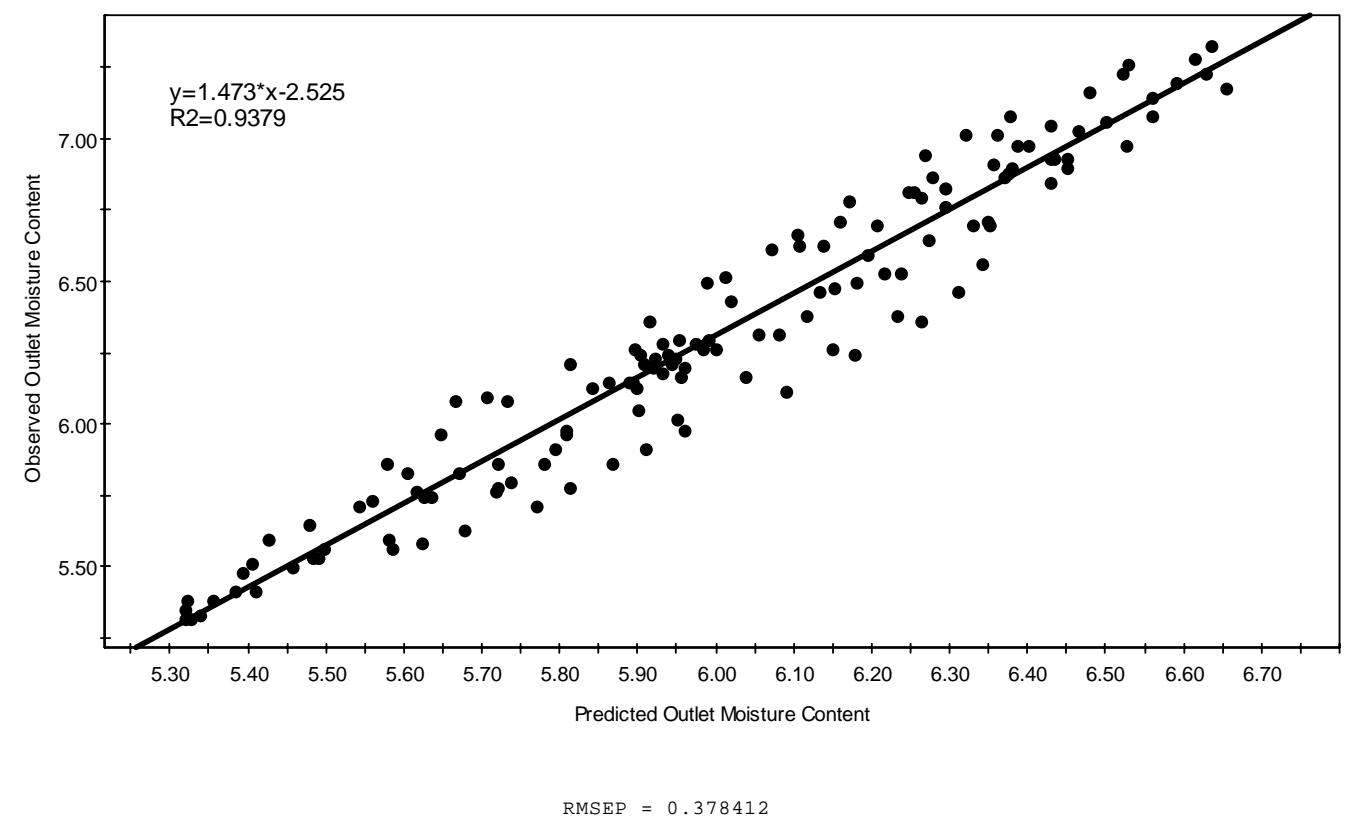

Figure 7.30 Outlet Moisture Content Predicted Scatter Plot

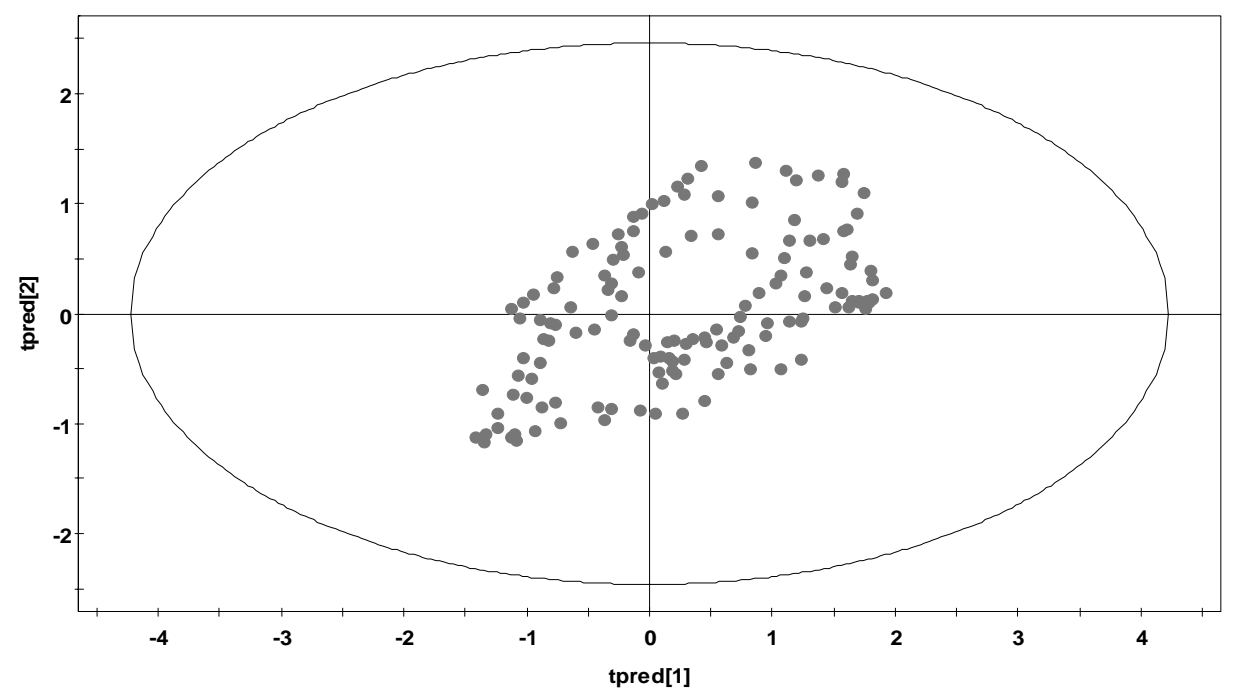

Figure 7.31 Score Plot Predicted Scores for Dryers and Heat Source

The DModX plot, Figure 7.32, shows the model did well with the first prediction set as there are no excursions above the D-Crit line. DModY, Figure 7.33, did have three 
distinct peaks indicating the model may be missing some of the variation in the data in modeling the response variable.

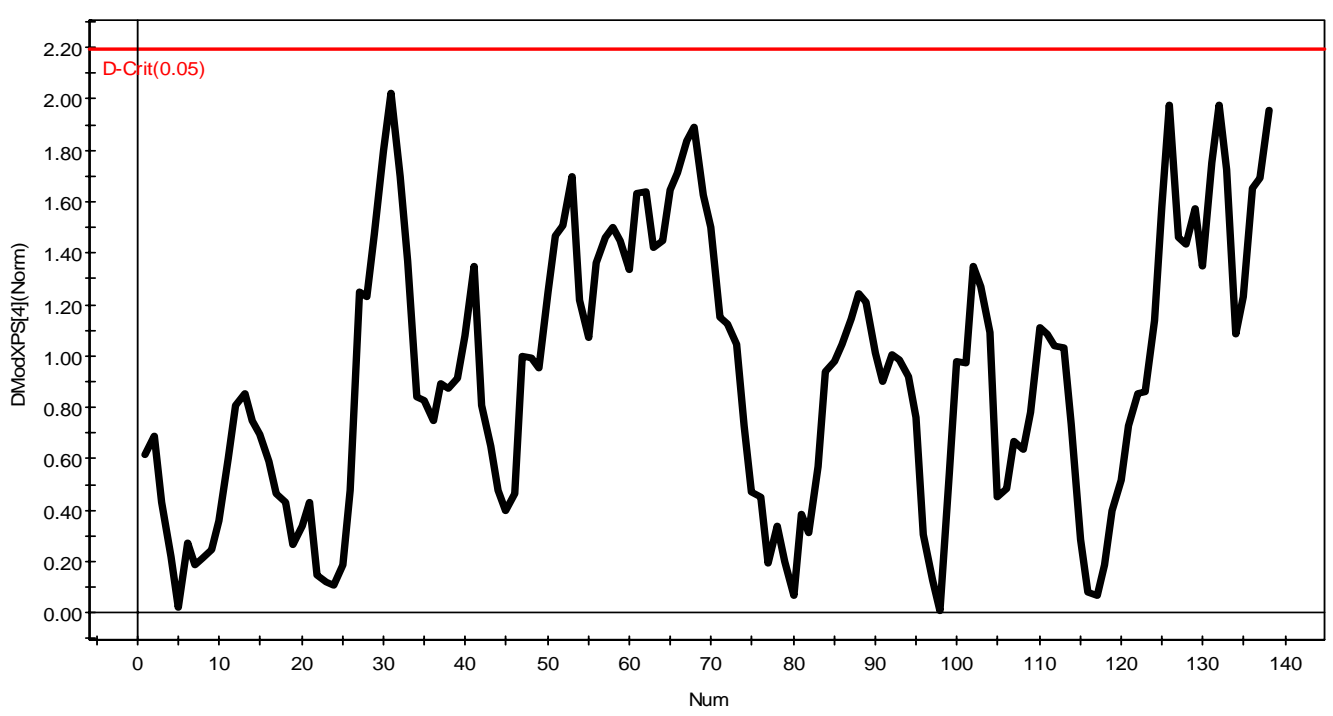

Figure 7.32 DModX for Prediction Set 1

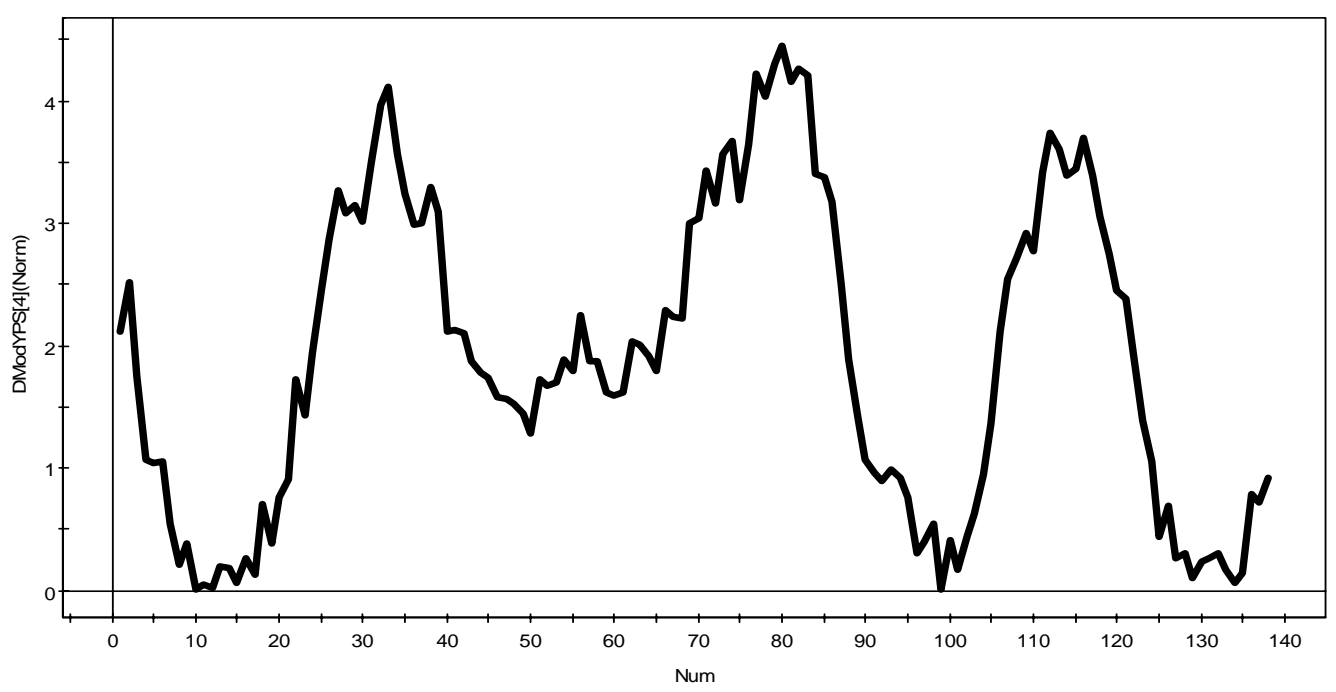

Figure 7.33 DModY for Prediction Set 1 


\subsubsection{Prediction Data Set 2}

The second external data set or prediction data set was collected from the process 44 days after the original data used to develop the model were collected.

The prediction scatter plot is shown in Figure 7.34. The root mean square error of prediction (RMSEP) is the standard deviation of the predicted residuals and is 0.557 for the third prediction data set. The $95 \%$ confidence interval is $\pm 1.11 \%$.

The model tended to over predict the moisture content. The model predicted $4.0 \%$ and the observed was $3.5 \%$ and predicted $6.0 \%$ and the observed was $5.2 \%$

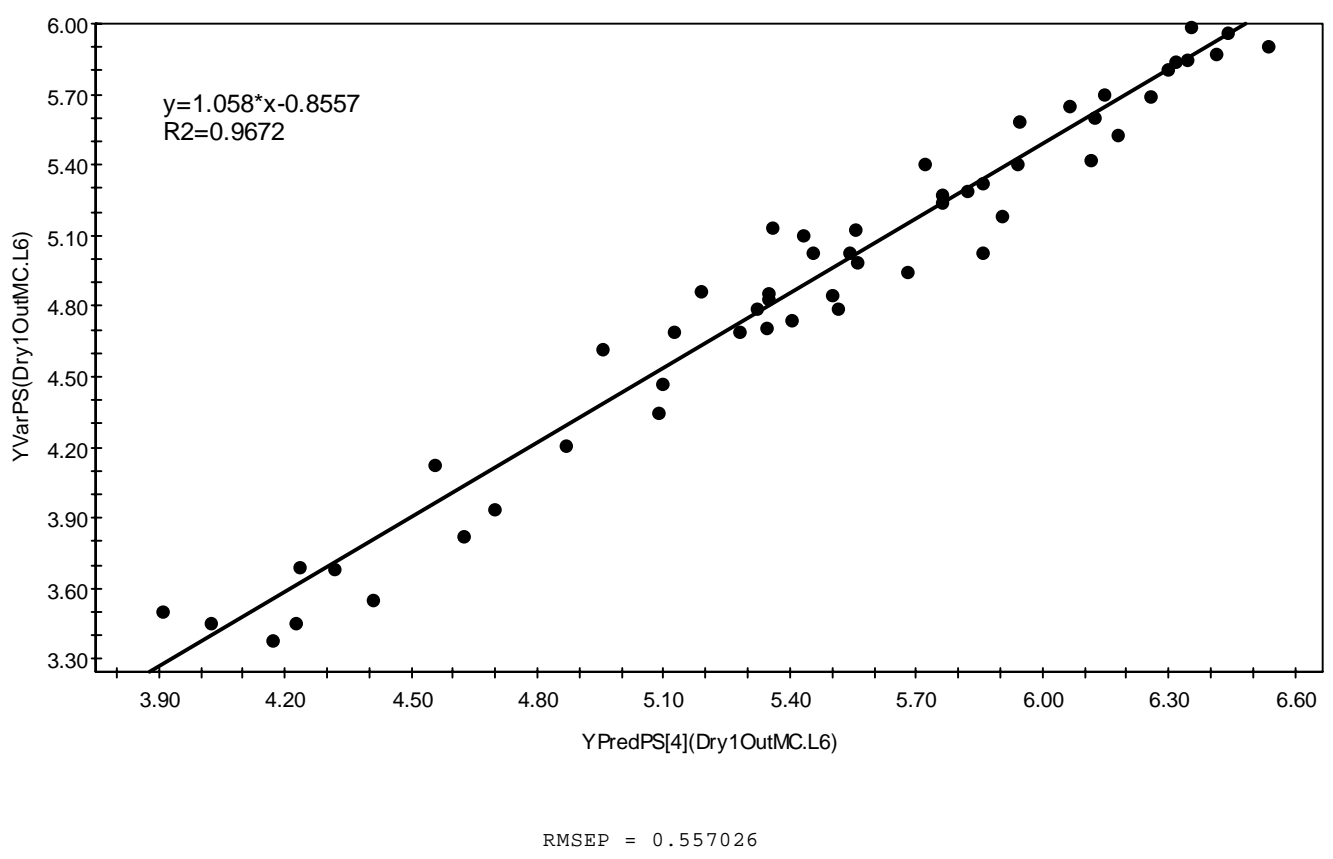

Figure 7.34 Outlet Moisture Content Predicted Scatter Plot - Prediction Set 2

The predicted scores were reviewed to see if the reason the predictions were off could be explained by the scores. For dryers and heat source turn down, the scores were in the center of the ellipse with no outliers. 
The DModX showed all the observations were within the D-Crit indicating they all belonged to the model.

\subsubsection{Model Calibration}

Processes may change over time and the model may be needed to be calibrated. To determine if calibration is needed, the predicted values are fitted to the observed values and the slope and intercept of the regression equation should be approximately 1.0 and 0 respectively. If there is a difference, then the predicted values may need to be "calibrated". This is accomplished by calculating new predicted values using the regression equation, slope and intercept.

Regression equations were determined for the prediction data sets (Figures 7.30 and 7.34). The slopes and intercepts were not 1.0 and 0.0 respectively. The second prediction set was close with a slope of 1.08 but the intercept was -0.86 . The intercept indicated an adjustment could be made by subtracting $0.86 \%$ from the predicted values to get a closer agreement. As discussed in section 7.3.2, the predicted values for the second predicted data set were higher than the observed, and calibration would improve these results.

Calibration may be needed with seasonal changes, raw material changes or even some minor process changes. If the process change is considerable a new model may be in order.

\subsubsection{Summary of Model Validation}

The two verification data sets used to validate the model showed the model is just like the infamous quote "All models are wrong some are just better than others." The PLS 
model is able to predict moisture contents within $1 \%$ but probably more importantly the model is able to identify variables that are moving and causing issues.

The model identified several opportunities for improving the drying process. One is improving the control loop for the heat source variables as seen in the departure in the second prediction data set. Another is improving the heat source turndown so it does not lag and lead the dryer temperatures. 


\section{MULTIVARIATE STATISTICAL PROCESS CONTROL}

"Variation in a process is natural; it should be expected. But, it is a wild beast that must be controlled." Gitlow and Gitlow

Statistical process control monitoring of OSB drying processes, has been attempted over the years in several different ways some more successful than others. Monitoring univariate data from a multivariate process requires a chart for each variable. The dryer PLS model identified five variables as influential so five univariate charts would be needed, one for each variable. Five charts with these variables are shown in Figure 8.1. This much data is difficult to monitor for just one dryer, and most OSB facilities have four dryers so the number quickly multiplies.

Monitoring variables one at a time results in losing interrelationships. "Variables of a multivariate process are often interrelated and form a correlated set. Since the variables do not behave independently of one another, they must be examined together as a group and not separately" (Mason and Young 2002). When two variables that are correlated are charted in separate control charts, the observations may be shown "in control" since they are inside the three sigma control limits. However, a different message is conveyed when the variables, A and B, are viewed in a scatter plot; now an observation shows up as outlier well away from the cluster of data of correlated data (Figure 8.2).

To have a successful multivariate control procedure Mason and Young (2002) comment, "The monitoring statistic should be easy to chart and helpful in identifying process trends." It has to be easy to determine the cause, i.e. what is the contributing variable, when out of control points occur. Having five separate univariate control charts as the control procedure is not as efficient as plotting multivariate statistics such as PCA or PLS 
scores, $\mathrm{t}$, or Hotelling's $\mathrm{T}^{2}$ based on such scores on just one chart. The chart might be a Shewhart, a Cusum, or even an EWMA.

PCA and PLS scores are new variables or latent variables that are summaries of the process variables. They are less noisy than the original process variables as they are weighted averages. In the case of PLS scores, the "process and quality variables are used together and the information residing in their correlation structure is extracted" (Eriksson et a. 2001). The Hotelling's $\mathrm{T}^{2}$ is a combination of all the scores for all components for a PCA or PLS model. A $\mathrm{T}^{2}$ plot then is a measure of how far way a new observation is from the center of the PLS model hyper plane. Instead of using an ellipse as is common practice when reviewing score plots in model development, the control chart can be plotted in time series order making it easier to see when the process goes out of control.

When a process does go out of control, e.g. the data goes above or below the control limits, questions need to be asked.

1. What changed in the process?

2. Did a variable change or did the correlation structure of the variables change?

3. Did the process or operating environment change?

$\checkmark$ Has a new operating procedure been implemented?

$\checkmark$ Is the process now operating outside the range in which the current model was developed?

$\checkmark$ Did a seasonal change occur?

$\checkmark$ Has there been a change in the equipment, has it been calibrated?

$\checkmark$ Has the process control loop been tuned?

4. Is a new model needed? 


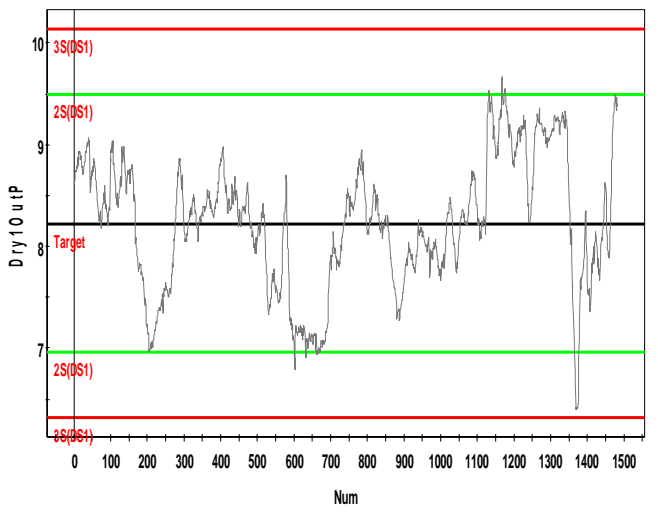

Out Pressure

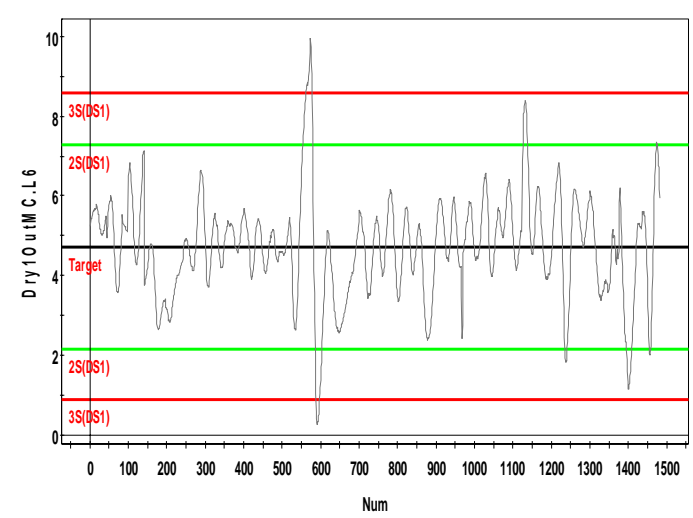

Outlet Temperature

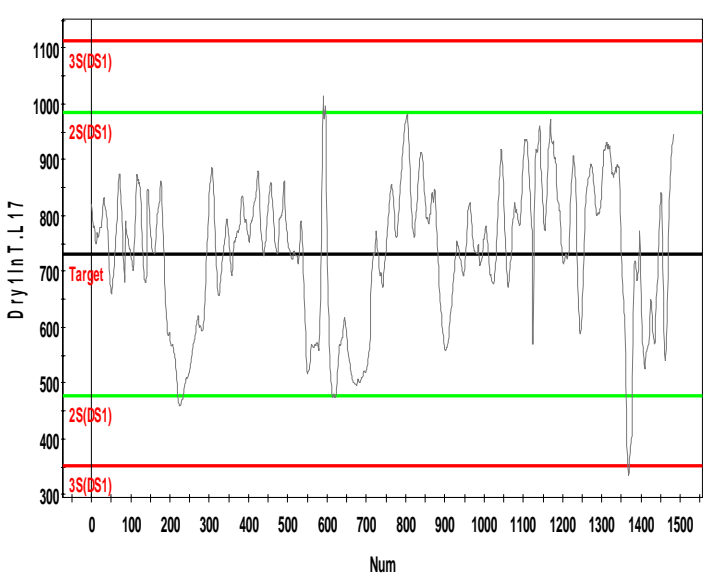

Inlet Temperature

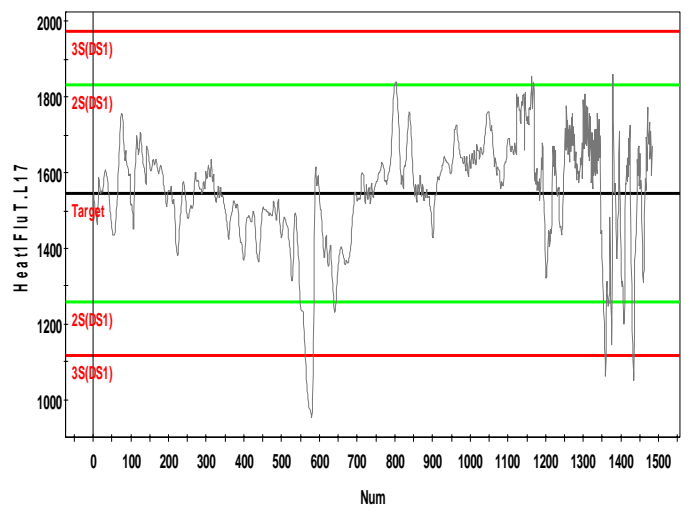

Flue Temperature

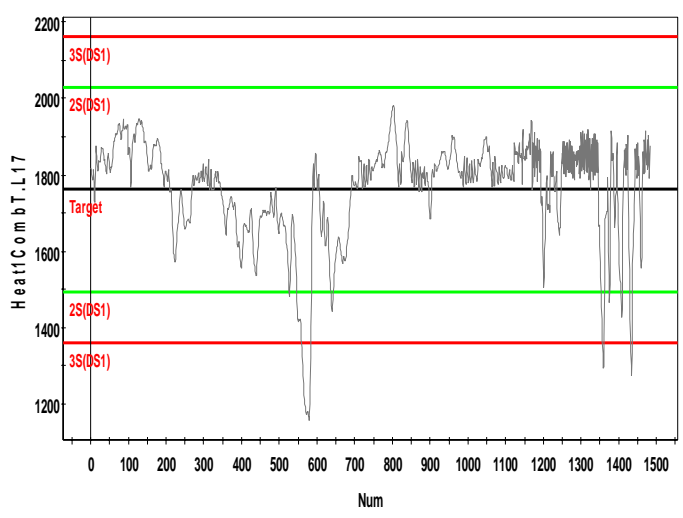

Combustion Temperature

Figure 8.1 Shewhart Charts for Each Influential Variable to Monitor Dryer \#1 

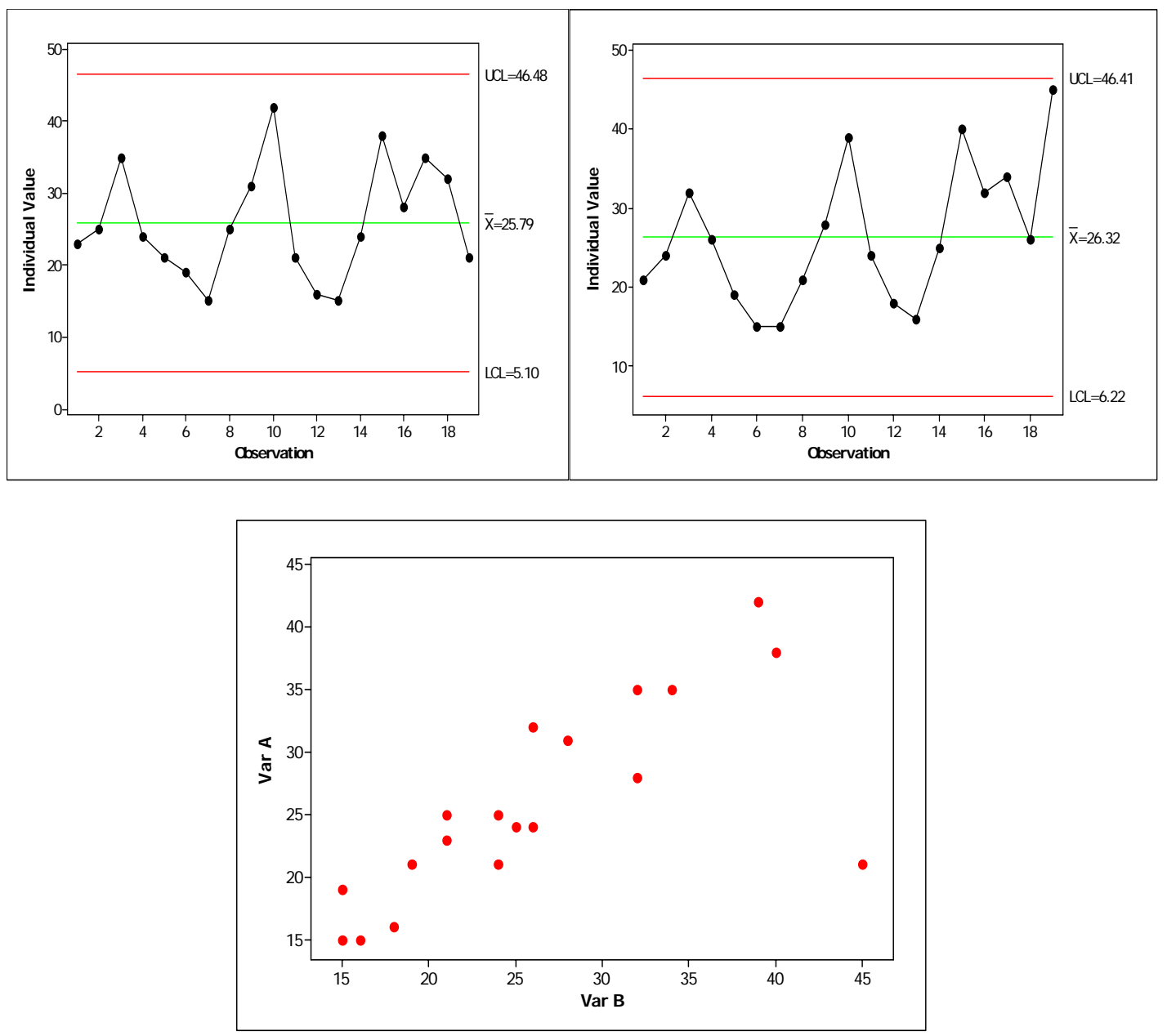

Figure 8.2 Control Charts of Correlated Variables. Scatter Plot Shows Outlier in Lower Right Corner

\subsection{MSPC Using OSB Drying Data and the PLS Dryer Model}

Three data sets from the OSB drying process were imported into SIMCA-P for the purpose of monitoring the process using the PLS model, developed in Chapter 6 and MSPC.

The first data set was from January 14, 2004 at 11:50 PM until January 15, 2004 12:30 AM. Multivariate control charts of the scores and Hotelling $\mathrm{T}^{2}$ were reviewed to see if the process was in control during that time. 
The scores for component one were plotted in a Shewhart plot, Figure 8.3. The process was in control for the dryers or first component of the PLS model. Reviewing what is happening with the model was accomplished by plotting Hotelling's $\mathrm{T}^{2}$ for using the Shewhart chart (Figure 8.4) The plot shows some oscillation but no observations were above the $\mathrm{T} 2 \mathrm{Crit} 95 \%$ line.

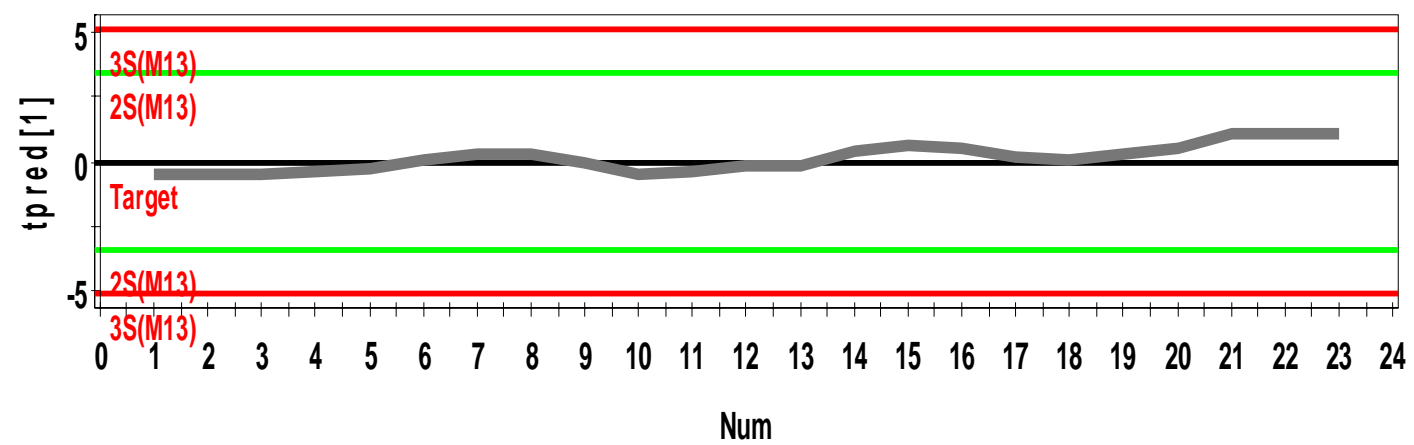

Figure 8.3 Shewhart Chart for Dryer Scores - Data Set Jan 14 \& 15, 2004

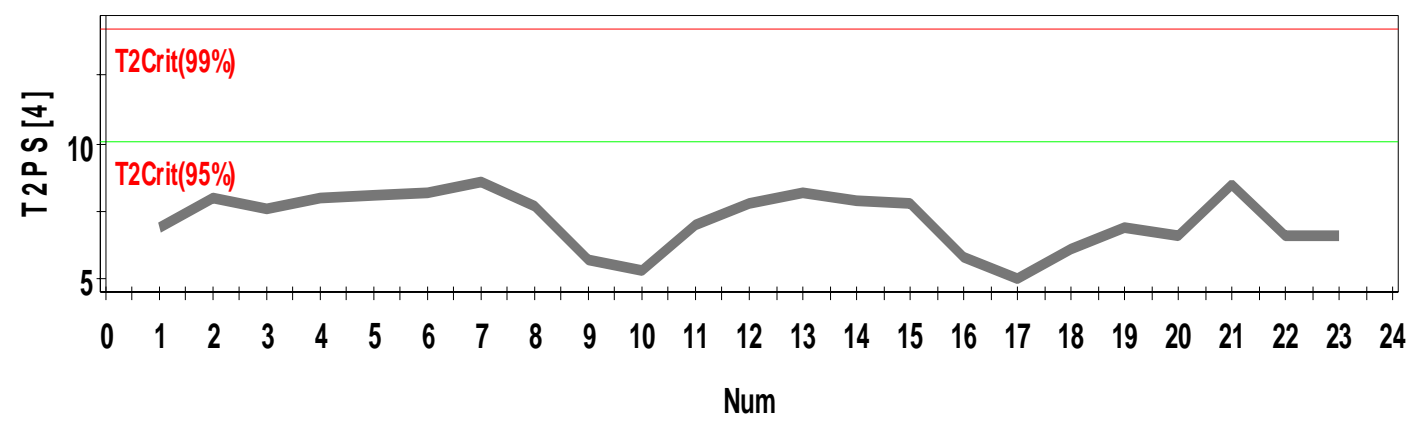

Figure 8.4 Shewhart Chart for Hotelling T ${ }^{2}$ - Data Set Jan 14 \& 15, 2004

The second data set was a 16 hour time period beginning on January 16, 2004. The data were pulled from the IP21 data historian to see how well the model predicted the moisture content and then how well was it controlled. The actual outlet moisture 
contents from dryer $\# 1$ are plotted along with the predicted moisture contents in a time series plot (Figure 8.5). The predicted values are the dashed lines and are tracking along with the observed. The model is slightly over predicting the moisture content, on average about $0.5 \%$.

The moistures were cycling throughout the time period but there was one time period about half way through that the moistures shot up to $10 \%$ and then all the way to zero and then rebounded.

Time Series of Predicted vs. Observed January 16, 2004

Solid Line is Observed / Dashed Line is Predicted

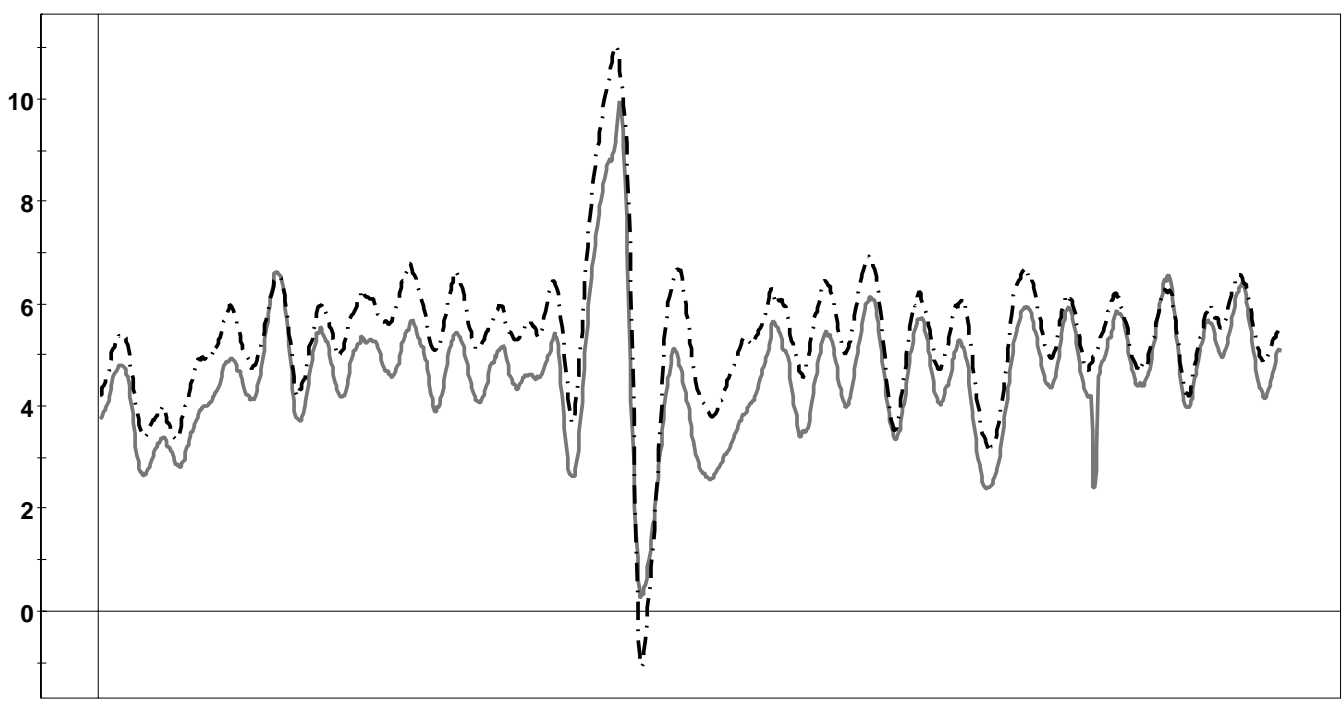

Figure 8.5 Outlet Moisture Contents Jan 16, 2004 00:00 hrs to 16:00 hrs

The Shewhart chart for dryer component scores is shown in Figure 8.6. It indicates these scores were generally in control except at the middle of the time period at the same time period that the observed moistures in Figure 8.5 went up and then down quickly. 


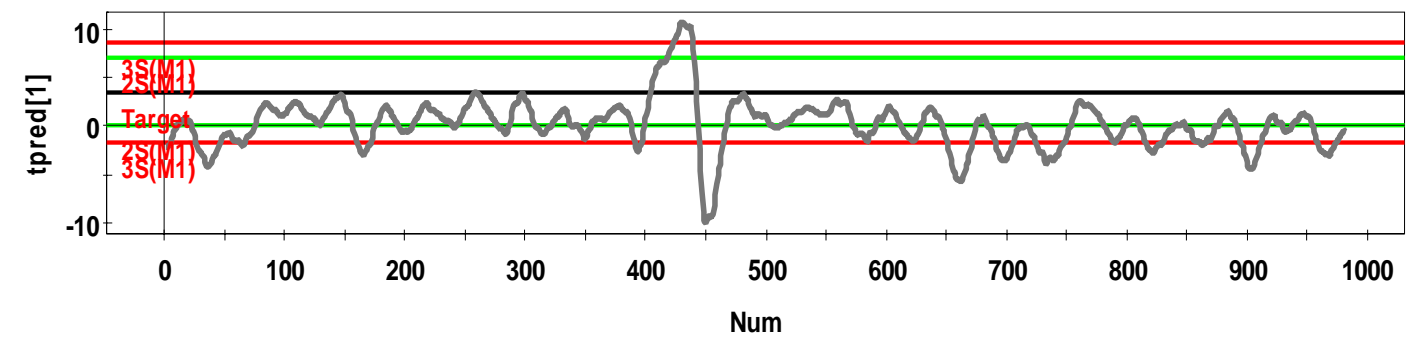

Figure 8.6 Shewhart Chart for Score t[1] - Data Set Jan 16, 2004

The large "swing" at the middle of the time period was reviewed using the contribution plot for the scores. The break in the correlation structure was due to the outlet pressure. The outlet pressure was lower than average at that time period. In looking back at all the process data, the weight scale was reduced to zero as the process was halted due to low bin levels. The halt make the outlet pressure drop off rapidly to less than 7" W.C. resulting in a break in the structure. In turn, the scores increased as did the moisture contents.

In the later part of the time period, the scores were trending down. A review of several contribution plots indicated the outlet pressure was lower than average while the dryer temperatures were higher than average thus lower scores and lower moisture contents.

A closer look at the five model variables revealed that all five were cycling well above and below the levels when the model data were collected. It could not be determined what the root cause was that caused the oscillation other than speculation. The other dryer that was not modeled was demanding heat from the heat source when the modeled dryer was not or vice versa. 
The final data set was imported from IP21 to study MSPC. The data was from February 6, 2004. The Hotelling $\mathrm{T}^{2}$ control chart, Figure 8.7, indicates the process was in control over the approximate three hour time period. The DModX plot shows no major outliers from the model (Figure 8.8). The actual vs. predicted moisture contents were plotted in a time series plot that shows the data are tracking well (Figure 8.9).

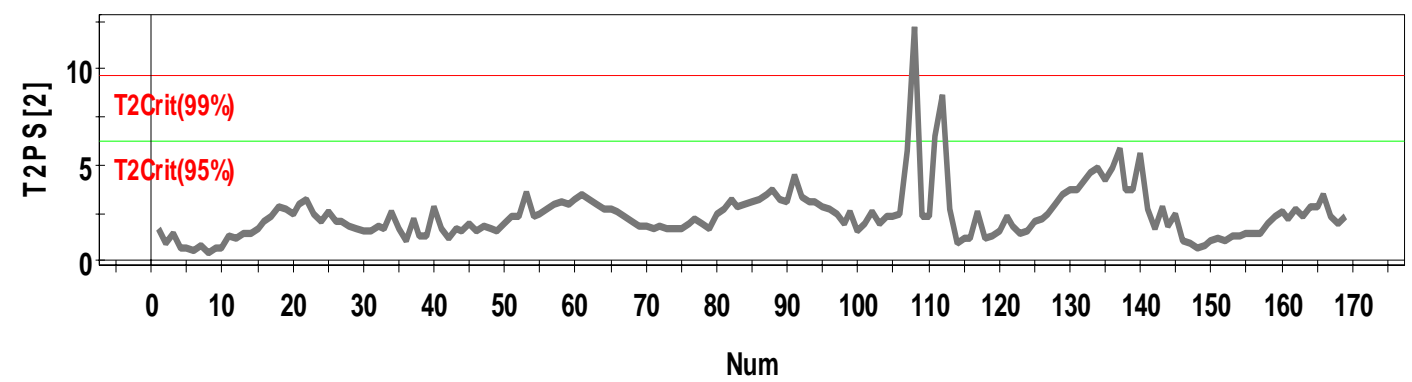

Figure 8.7 Hotelling T² Control Chart for Jan 21, 2004 12:05 AM to 1:05 AM

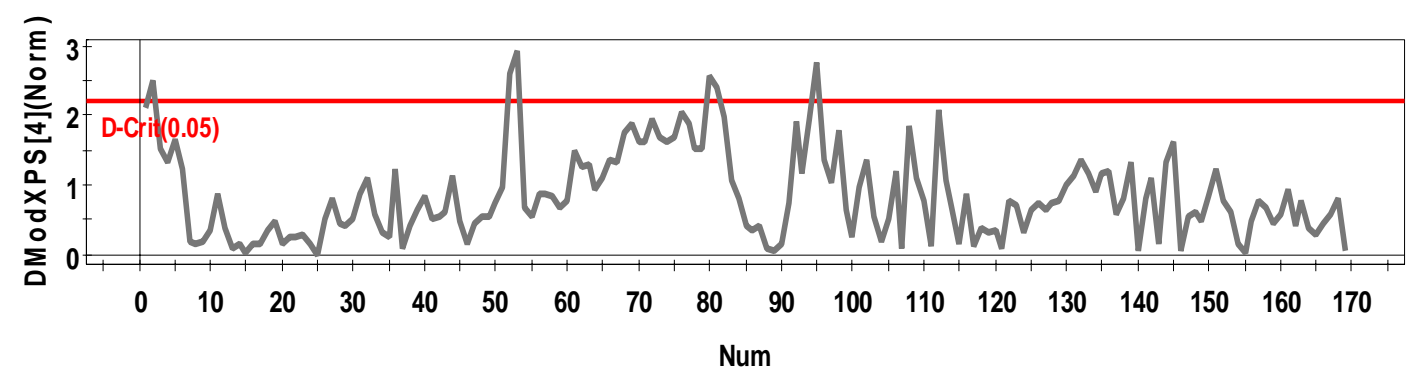

Figure 8.8 DModX Plot 
Time Series of Predicted versus Observed Feb 6, 2004

Solid Line is Observed / Dashed Line is Predicted

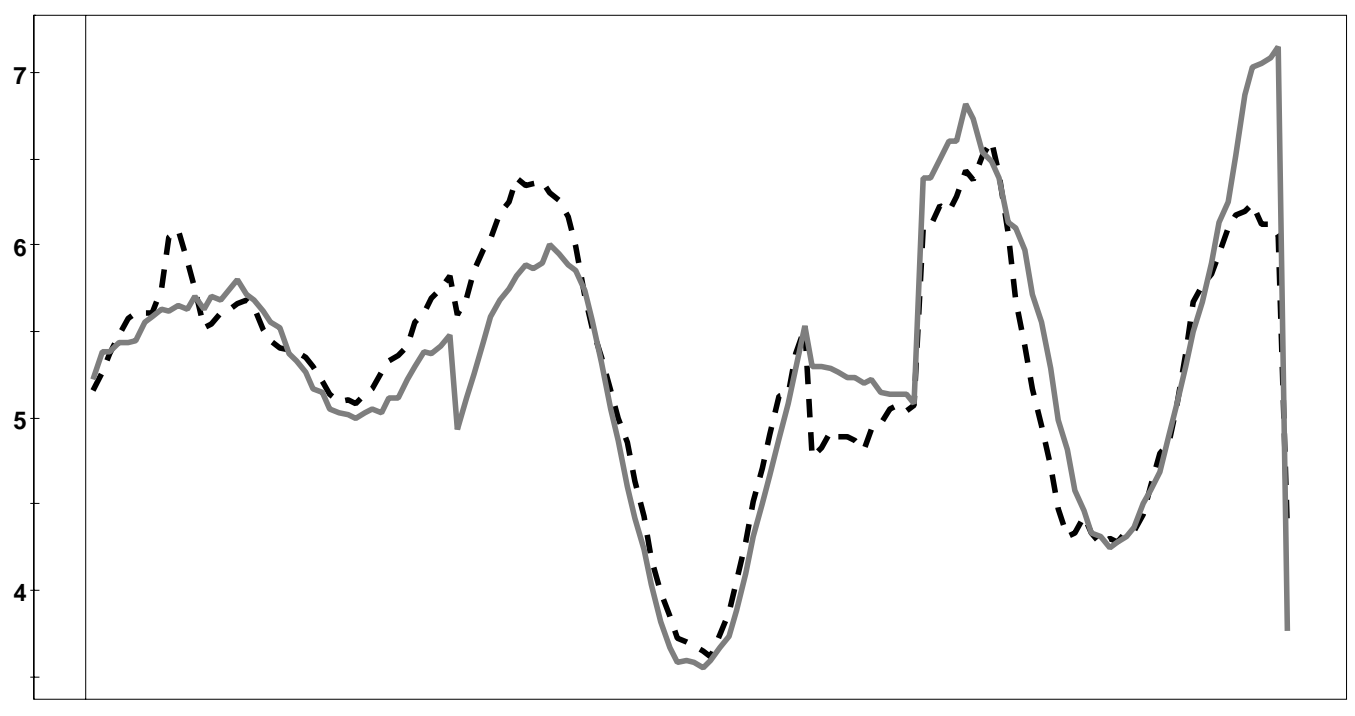

Figure 8.9 Plot of Actual vs. Predicted MC for Feb 6, 2004 data

\subsection{Monitoring and Updating the Dryer Model}

A model is as good as the data that was used to develop it. If the data changes due to, for example, process changes, input material changes, or seasonality effects, the model needs to be updated. Eriksson et al. (2001) recommends continually adding sample observations to the model on a regular basis. This could be as often as daily or weekly. It could be based on a percent of the data monitored with the model.

On a regular basis when using the model for process monitoring or prediction, the PLS diagnostic tools should be used to understand whether new observations conform to the model or not. Should a new observation for some reason be different from the model training set, it is risky to make predictions. The model residuals using DModX makes it possible to classify a new observation as similar or dissimilar to the training set. In turn 
the DModX gives an insight into model relevance and whether the process has changed enough that the model needs to be updated and revalidated. 


\section{SUMMARY AND CONCLUSION}

"If quality improves, productivity increases. To increase productivity, management must stress quality not quantity.” Gitlow and Gitlow

\subsection{Summary}

The objective of this research was to characterize the oriented strand board manufacturing process so continuous improvement would be possible. The investigation included the development of an OSB mill process flow model, and OSB mill model, an OSB drying model, and a process MSPC tool.

The OSB mill process flow model was the process flow diagram outlining each major processing step so continuous improvement opportunities can be identified. The mill model was developed using Excel ${ }^{\circledR}$-based computer program. It answered "what ifs" or "trade-off" questions by operations to help understand the ramifications of changes to the processes through the continuous improvement efforts.

The dryer model was a multivariate PLS model using SIMCA-P software for model development and validation. Five process variables were selected as the "most influential" predictors for outlet moisture content. These five variables were reduced to two factors / components - dryer and heat source - for the PLS model.

The process MSPC tool used component scores and Hotelling $\mathrm{T}^{2}$ to monitor the process variables.

\subsection{Conclusions}

The mill model facilitated dialogue about trade-off's and improved the process overall. Two examples where the mill model assisted operations in arriving at sound decisions were the impact of knife projection and knife changes on strand production and the effect of fines content on wood costs. It was also found with drying operations that a 
series of time series trends, mass balance, provided operators with visual evidence of effect the process changes they made had on productivity and quality - specifically surging in the process and its effect on moisture content.

The dryer model's five most influential variables effectively predicted outlet moisture content and provided new insight into the intricacies of the process. The conveyor moisture meter system is being installed on the other three dryers along with the rate change program for feed rate. The effect of the combustion and flue temperatures on the outlet moistures has raised awareness on the importance of heat source control. To this regard PID loop tuning is being reviewed and a capital project is being engineered to improve the blend air chamber temperature control to reduce the heat source turndown influence on outlet moistures.

An on-line MSPC tool is being investigated to provide operators with quick feedback on the drying process.

The PLS technique demonstrated the "power" to "peel the onion" to determine the key process variables to define the variation in the process. It will be used to investigate the formline and it variables to understand the panel horizontal and vertical density profile. 


\section{RECOMMENDATONS FOR FURTHER RESEARCH}

"I'm like a child. I always ask the simplest questions." Albert Einstein

The research reported on in this dissertation may be improved on by:

$\checkmark$ Knowing the amount of water or water load to be removed by the dryer system could improve the accuracy of the predicted outlet moisture content. A new experimental inlet moisture content meter using nuclear magnetic resolution (NMR) might be used to determine the water loading.

$\checkmark$ Developing a "system approach" to modeling dryers that have a common heat source is necessary. The impact the other not modeled dryer had on "swinging" the heat source was not recognized initially. Common dryers need to be modeled concurrently.

$\checkmark$ Increasing the scale of the factor levels for both outlet temperature and wood feed rates to push "the envelop" and see if the influential variables change. Shortly after the research data was modeled, the outlet temperature set point was increased well past the upper level studied. This was due to deterioration in the heat source plenum and the model did not predict as well. So expanding the "normal" operating levels can be explorer to improve the model's usefulness.

This research presented with PLS might be extended to other steps in the OSB process.

$\checkmark$ Model the blending, forming, and pressing steps to predict panel properties such as internal bond and post flex.

$\checkmark$ Model the strength properties to determine the relationships among the 10 plus properties tested for quality assurance. 


\section{BIBLIOGRAPHY}

Alwan, L.C. and H.V. Roberts. 1988. Time-Series Modeling for Statistical Process Control. J of Bus and Economic Statistics 6(1): 87-95.

Bagshaw, M. and R.A. Johnson. 1975. The Effect of Serial Correlation on the Performance of CUSUM Tests II. Technometrics. 17: 73-80.

Box, G.E.P. and A. Luceno. 1997. Statistical Control by Monitoring and Feedback Adjustment. John Wiley \& Sons, Inc. New York. 327 pp.

Box, G.E.P., G.M. Jenkins, and G.C. Reinsel. 1994. Time Series Analysis, Forecasting and Control. $3^{\text {rd }}$. Prentice-Hall. Upper Saddle River, N.J. 598 pp.

Brown, H. P., A. J. Panshin, and C. C. Forsaith. 1952. Textbook of Wood Technology Volume II. McGraw-Hill Book Company. NY., NY. 783 pp.

Davis, C.R. 1997. Personal communication - concerning PF resins and the impact of process variables on the bonding efficacy. Georgia Pacific Resin Inc R\&D Chemist.

Deming, W.E. 1986. Out of the Crisis. Massachusetts Institute of Technology. Cambridge, MA. 507 pp.

Ducharme, M. 2003 Personal communication concerning findings from a study conducted at Slave Lake, Alberta OSB plant. Weyerhaeuser Technical Director.

Eriksson, L., E. Johansson, N. Kettaneh-Wold, and S. Wold. 1999. Introduction to Multiand Megavariate Data Analysis using Projection Methods (PCA \& PLS). Umetrics AB. Sweden. 490 pp.

Eriksson, L., E. Johansson, N. Kettaneh-Wold, and S. Wold. 2001. Multi- and Megavariate Data Analysis. Umetrics AB. Sweden. 533 pp.

Friedman, S.J. and W.R. Marshall. 1949. Studies in Rotary Drying. Part II-Heat and Mass Transfer. Chemical Engineering Progress. 45:573-588.

Gilbert, K.C., K. Kirby, and C.R. Hild. 1997. Charting Autocorrelated data: guidelines for practitioners. Quality Engineering. 9(3): 367-382.

Ginzburg, A.S. 1958. Grain Drying and Grain Dryers. Office of Technical Services, U.S. Department of Commerence, Washington, D.C. 304 pp.

Goldratt, E. 1992. The Goal. North River Press. Great Barrington, Ma. 337 pp. 
Harris, T.J. and W.H. Ross. 1991. Statistical Process Control for Correlated Observations. The Canadian J of Chemical Engineering 69: 48-57.

Hunter, J.S. 1986. The Exponentially Weighted Moving Average. J of Quality Technology 18(4): 203-210.

Honda, B. G. 1984. Dryer Control for Medium Density Fiberboard. Technical Report Project No. 045-2601. Weyerhaeuser Co. pp. 32.

Jackson, J.E. 1991. A User Guide to Principal Components. John Wiley and Sons. New York, NY. XXX pp.

Johnson, R.A. and M. Bagshaw. 1974. The Effect of Serial Correlation on the Performance of CUSUM Tests. Technometrics 16: 103-112.

Kachigan, S. K. 1991. Multivariate Statistical Analysis, A Conceptual Introduction. Radius Press. New York. 303 pp.

Kamke, F.A. 1983. Engineering Analysis of a Rotary Dryer: Drying of Wood Particles. Ph.D. Dissertation, Oregon State University, Corvallis, OR.

Kume, H. 1985. Statistical Methods for Quality Improvement. Association for Overseas Technical Scholarship. Tokyo. 231 pp.

Lindgren, F., P. Geladi, and S. Wold. 1993. The Kernel Algorithm for PLS. J. of Chemometrics. Vol 7:45-59.

Longnecker, M.T. and T.P. Ryan. 1992. Charting Correlated Process Data. Technical Report 166. Texas A.M. University Department of Statistics.

Maloney, T.M. 1993. Modern Particleboard \& Dry-Process Fiberboard Manufacturing. Miller Freeman Inc. San Francisco, CA. 681 pp.

Manugistics, 1999. Statgraphics Plus for Windows, Version 5.0. Rockville, Md.

Martens, H. and M. Martens. 2001. Multivariate Analysis of Quality an Introduction. John Wiley and Sons, LTD. New York. 445 pp.

Martens, H. and T. Naes. 1989. Multivariate Calibration. John Wiley and Sons, LTD. New York. 419pp.

Mason, R.L. and J.C. Young. 2000. Interpretive Features of a $\mathrm{T}^{2}$ Chart in Multivariate SPC. Quality Progress 33(4): 84-89.

Mason, R.L. and J.C. Young. 2002. Multivariate Statistical Process Control With Industrial Applications. ASA-SIAM. Philadelphia, PA. 263 pp. 
Mason, R.L., N.D. Tracy, and J.C. Young. 1995. Decomposition of $\mathrm{T}^{2}$ for Multivariate Control Chart Interpretation. Journal of Quality Technology 27(2): 99-108.

Mason, R.L., N.D. Tracy, and J.C. Young. 1997. A Practical Approach for Interpreting Multivariate $T^{2}$ Control Chart Signals. Journal of Quality Technology 29(3):396-406.

McCormick, P.Y. 1962. Gas Velocity Effects of Heat Transfer in Direct Heat Rotary Dryers. Chemical Engineering Progress. 58(6):57-61. (Referenced by Kamke 1983)

Miller, C.O., B.A. Smith, and W.H. Schuette. 1942. Factors Influencing the Operation of Rotary Dryers. Transactions, American Institution of Chemical Engineers. 38:841864. (Referenced by Kamke 1983)

Minitab. 2003. MINITAB® Statistical Software, Release 14. State College, PA.

Montgomery, D.C. 1997. Introduction to Statistical Quality Control, $3^{\text {rd }}$ ed. John Wiley \& Sons Inc. U.S.A.

Montgomery, D.C. and C.M. Mastrangelo. 1991. Some Statistical Process Control Methods for Autocorrelated Data. J of Quality Technology 23(3): 179-193

Mujumdar, A.S. 1987. Handbook of Industrial Drying. Marcel Dekker. New York. 948 pp. 948.

National Institute of Standards and Technology. 1992. PS2-92 Performance Standard for Wood-based Structural-use Panels. Government Printing Office. Washington, D.C. 26 pp.

Noffsinger, J.R. and B.R. Anderson. 2002. Effect of Autocorrelated Data on Composite Panel Production Monitoring and Control: A Comparison of SPC Techniques. Forest Product J. 52(3):60-67.

Paulin, R. 2003. Personal Communication concerning pMDI resin and the effect strand moisture content has on bonding efficacy. Huntsman Technical Service Specialist.

Panel World. 2004. Structural Panel Production Set Record in 2003 at 40.9 Billion. Panel World. 45(2): 6.

National Institute of Standards and Technology. 1992. PS2-92 Performance Standard for Wood-based Structural-use Panels. Government Printing Office. Washington, D.C. 26 pp. 
Seaman, W.C. and J.R. Mitchell. 1954. Analysis of Rotary Dryer and Cooler Performance. Chemical Engineering Progress. 50(9):49-56 (Referenced by Kamke 1983)

Sharples, K., P.G. Glikin, and R. Warne. 1964. Computer Simulation of Rotary Driers. Transactions, Institute of Chemical Engineers. 42:T275-T284. (Referenced by Kamke 1983)

Shinskey, F.G. and D. Fadum. 1980. Saving Energy Through Better Control of Continuous and Batch Dryers. Control Engineering. March 1980. (Referenced by Kamke 1983)

Skaar, C. 1972. Water in Wood. Syracuse University Press. Syracuse. 218 pp.

Snee, R.D. 2002. Develop Useful Models. Quality Progress. 35(12): 94 - 98.

Tal, J. 2001. Reading Between the Numbers. McGraw-Hill. N.Y., N.Y. 285 pages.

Thorne, B. 1979. The Computer Simulation of the Rotary Drying Process. Ph.D. Thesis, University College, Dublin, Ireland. (Referenced by Kamke 1983)

Traub, D. A. 2000. Let's Talk Control, Part 1. www.process-heating.com (October 12, 2000). 2 pp.

Trout, J. 2000. Differentiate or Die. John Wiley \& Sons. N.Y., N.Y. 230 pp.

Umetrics. 2000. Multivariate Data Analysis and Modeling Three Day Course. Umetrics, Inc. Kinnelon, N.J. 180 pp.

Umetrics 2001. SIMCA-P Version 9.0. Sweden.

Umetrics 2003 Training Course on SIMCA-P. Seattle WA. Umetrics, Inc. Kinnelon, N.J. 220 pages.

Van Krevelen, D.W. and P.J. Hotijzer. 1949. I. Soc. Ch. Ind. 68,59;59 (Referenced by Mujumbar 1987)

Vasilopoulos, A.V. and A.P. Stamboulis. 1978. Modification of Control Chart Limits in the Presence of Data Correlation. J of Quality Technology. 10(1): 20-30.

Wardell, D.G., H. Moskowitz, and R.D. Plante. 1994. Run-Length Distributions of Special-Cause Control Charts for Correlated Processes. Technometrics 36: 3-17.

Young, T.M. and P.M. Winistofer. 1999. Statistical Process Control and the Forest Products Industry. For Prod J. 49(3): 12-17. 
Zhang, N.F. 1997. Detection Capability of Residual Chart for Autocorrelated Data. J of Applied Statistics. 24: 475-492.

Zhang, N.F. 1998. A Statistical Control Chart for Stationary Process Data. Technometrics 40(1): $24-38$ 


\section{OSB Mill Model}

by John R. Noffsinger Jan 2004

This Model is useful in helping to guide mill productivity and cost decisions.

Please note since the model is still under development there may be errors. If you get an answer that looks questionable please email john.noffsinger@weyerhaeuser.com or see him with your question or concern.

\section{$\underline{\text { Procedures }}$}

The model uses input variables found in the Input Data - Recipe worksheet.

The Input Data - Recipe worksheet has all the process steps and the various parameters beginning with cost for raw materials and proceeding down the worksheet the various process steps begining with stranding,blending pressing etc.

At the bottom are the recipes for the various products produced.

Do Not change any of the data on the other worksheets as they are all driven by values found in the Input Data - Recipe worksheet and by formulas found in the various product worksheets

\section{$\underline{\text { Various Worksheets }}$}

The Summary Rates worksheet sumnmarizes the findings for the model for the various products

The Raw Material - Wood worksheet is the input screen for the species processed by the mill. It uses compaction ratio to determine panel density and the average density of the species mix that is then used in the LSS -Strander worksheet.

The LSS-Strander worksheet contains the calculations for the Pallmann stranders - weight per stroke, max strokes per hour, etc.

The Bin Levels worksheet contains information on the maximum weight the bins can hold along with the length of time to theoretically unload a bin.

The Drying and Blending worksheets contain summary information related to these parts of the process.

The Product workheets contain the formulas in the model 


\begin{tabular}{|c|c|c|c|c|c|c|c|}
\hline \multirow[t]{6}{*}{ COSTS } & & & Solids & Price Per Pound & Date of Con & & \\
\hline & LPF $155 c 42$ & & & .5 & 7/22/2 & & \\
\hline & MDI & & & & 7/22/2 & & \\
\hline & Wax & & & & 7/22/2 & & \\
\hline & Release Agent & & & & $7 / 22 / 2$ & & \\
\hline & Average Wood Cost per Ton & & & & 20 & & \\
\hline \multirow[t]{24}{*}{ LSS } & Tons per Truck & & & & 23.5 tons & & \\
\hline & Strander Chamber/conveyor \% filled & ated chamber usage & & & 0.53 & & \\
\hline & Average Density based on species $m$ & & & & $48.93 \mathrm{pcf}$ (weghted average & & \\
\hline & Void Space due to log quality (3) & & & & 0.45 percent & & \\
\hline & Bulk Density of Wood & & & & $26.9115 \mathrm{pcf}$ (weghted average & & \\
\hline & Mill Determined Advance Time Per S & & & see recipe below & & & \\
\hline & Dead time Per Stroke & & & & 8 seconds & & \\
\hline & Knife Changes per shift per strander & & & & 4 changes / strander & & \\
\hline & Knife Change time & & & & 30 minutes & & \\
\hline & Strander Chamber Height & & & & 43.3 inches & & \\
\hline & Strander Chamber Width & & & & 66.9 inches & & \\
\hline & Strander Chamber Depth & & & & 28.5 inches & & \\
\hline & Strander RPM & & & & $350 \mathrm{rpm}$ & & \\
\hline & Strander Asset Utilization & & & & $0.87 \%$ Uptime & & \\
\hline & Strander Cut Time -- Carriage Advan & & & & 20 seconds avg for all pr & & \\
\hline & Strander "dead time" & & & & 8 seconds & & \\
\hline & Strander Total Knives in Ring & & & & 46 count & & \\
\hline & Strander Maximum Stroke Count & & & & 1000 count & & \\
\hline & Strander Average Strokes per Shift / & (Historical Average) & & & 778 strokes & & \\
\hline & Wood MC & & & & 0.7386 Depends on species & & \\
\hline & Bark & & & & 0.09 & & \\
\hline & Fines at Stranders & & & & 0.35 & & \\
\hline & Fines Screened Out Efficiency & Surface & & & 0.6 & & \\
\hline & & Core & & & 0.1 & & \\
\hline \multirow[t]{12}{*}{ Forming } & Mat Size Under Formers and After S & & & & & & \\
\hline & Deckel Chain Width - Forming Width & & & & & & \\
\hline & Mat Length & & & & & & \\
\hline & Length after FCOS & & & & & & \\
\hline & FCOS Removal - End & & & & & & \\
\hline & Side Trim Removal / Side & & & & & & \\
\hline & Side Hog Saw Removal / side & & & & & & \\
\hline & Hog saw Removal End & & & & & & \\
\hline & Pass Saw Removal / Side & & & & & & \\
\hline & Second Pass Saw Removal / End & & & & & & \\
\hline & Length After Second Pass Saw & & & & & & \\
\hline & Width After First Pass Saw & & & & & & \\
\hline \multirow[t]{4}{*}{ SPW } & Panel Size at Toledo & & & & & & \\
\hline & Width & & & & & & \\
\hline & Length & & & & & & \\
\hline & 4 by 8 sizes & 47. & 95. & & & & \\
\hline Losses & Losses (kerf and trim) & 0.0 & & Base on ARC study data & & & \\
\hline Pressing & Product & Density & Out of Press Thk & Press Cycle & Out of Press MC & AU & \\
\hline \multirow[t]{25}{*}{ Recipes } & $7 / 16$ & 40 & 0.438 & 20 & 0.03 & 1 & \\
\hline & $1 / 2$ & 40 & 0.5 & 20 & 0.03 & 1 & \\
\hline & $19 / 32$ & 40 & 0.594 & 20 & 0.03 & 1 & \\
\hline & $23 / 32$ & 40 & 0.719 & 20 & 0.03 & 1 & \\
\hline & 23/32 EG & 40 & 0.719 & 20 & 0.03 & 1 & \\
\hline & $3 / 4 \mathrm{P} 3$ & 40 & 0.75 & 20 & 0.03 & 1 & \\
\hline & $7 / 8 \mathrm{P} 3$ & 40 & 0.825 & 20 & 0.03 & 1 & \\
\hline & & DeadTime & Surface to Core Ratio & Surface Resin & Core Resin & Wax Surf & Wax Core \\
\hline & $7 / 16$ & 60 & 0.5 & 0.01 & 0.01 & 0.01 & 0.01 \\
\hline & $1 / 2$ & 60 & 0.5 & 0.01 & 0.01 & 0.01 & 0.01 \\
\hline & $19 / 32$ & 60 & 0.5 & 0.01 & 0.01 & 0.01 & 0.01 \\
\hline & $23 / 32$ & 60 & 0.5 & 0.01 & 0.01 & 0.01 & 0.01 \\
\hline & 23/32 EG & 60 & 0.5 & 0.01 & 0.01 & 0.01 & 0.01 \\
\hline & $3 / 4 \mathrm{P} 3$ & 60 & 0.5 & 0.01 & 0.01 & 0.01 & 0.01 \\
\hline & $7 / 8 \mathrm{P} 3$ & 60 & 0.5 & 0.01 & 0.01 & 0.01 & 0.01 \\
\hline & & & & Strander & Strander & & \\
\hline & & Form MC Surf & From MC Core & Cut Time & Cycle Time & & \\
\hline & $7 / 16$ & 0.1 & 0.05 & 20 & 28 & & \\
\hline & $1 / 2$ & 0.1 & 0.05 & 20 & 28 & & \\
\hline & $19 / 32$ & 0.1 & 0.05 & 20 & 28 & & \\
\hline & $23 / 32$ & 0.1 & 0.05 & 20 & 28 & & \\
\hline & 23/32 EG & 0.1 & 0.05 & 20 & 28 & & \\
\hline & $3 / 4 \mathrm{P} 3$ & 0.1 & 0.05 & 20 & 28 & & \\
\hline & $7 / 8 \mathrm{P} 3$ & 0.1 & 0.05 & 20 & 28 & & \\
\hline & & & Avg Cut & 20 & & & \\
\hline
\end{tabular}




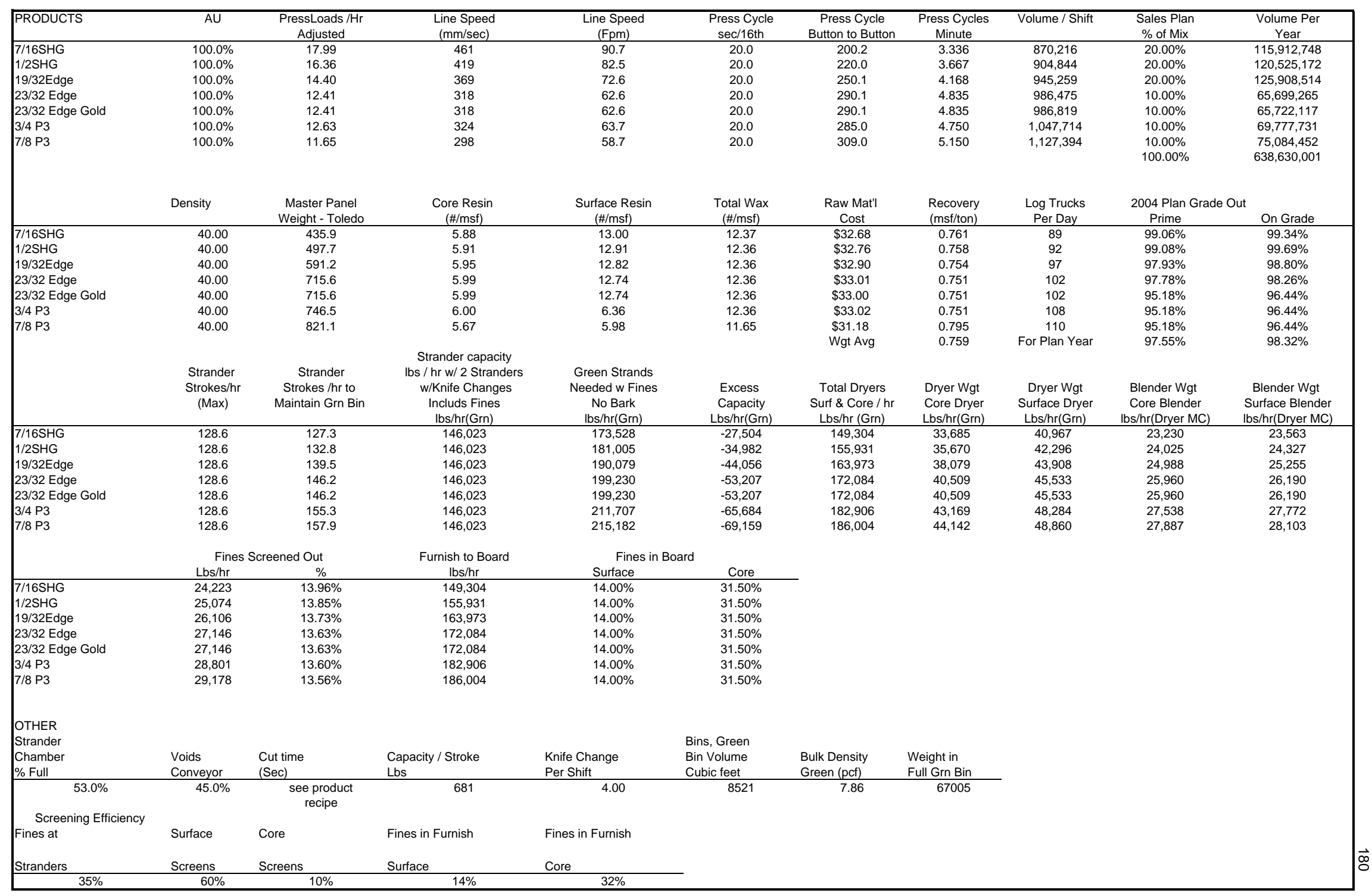

OSB Mill Model Summary Rates 


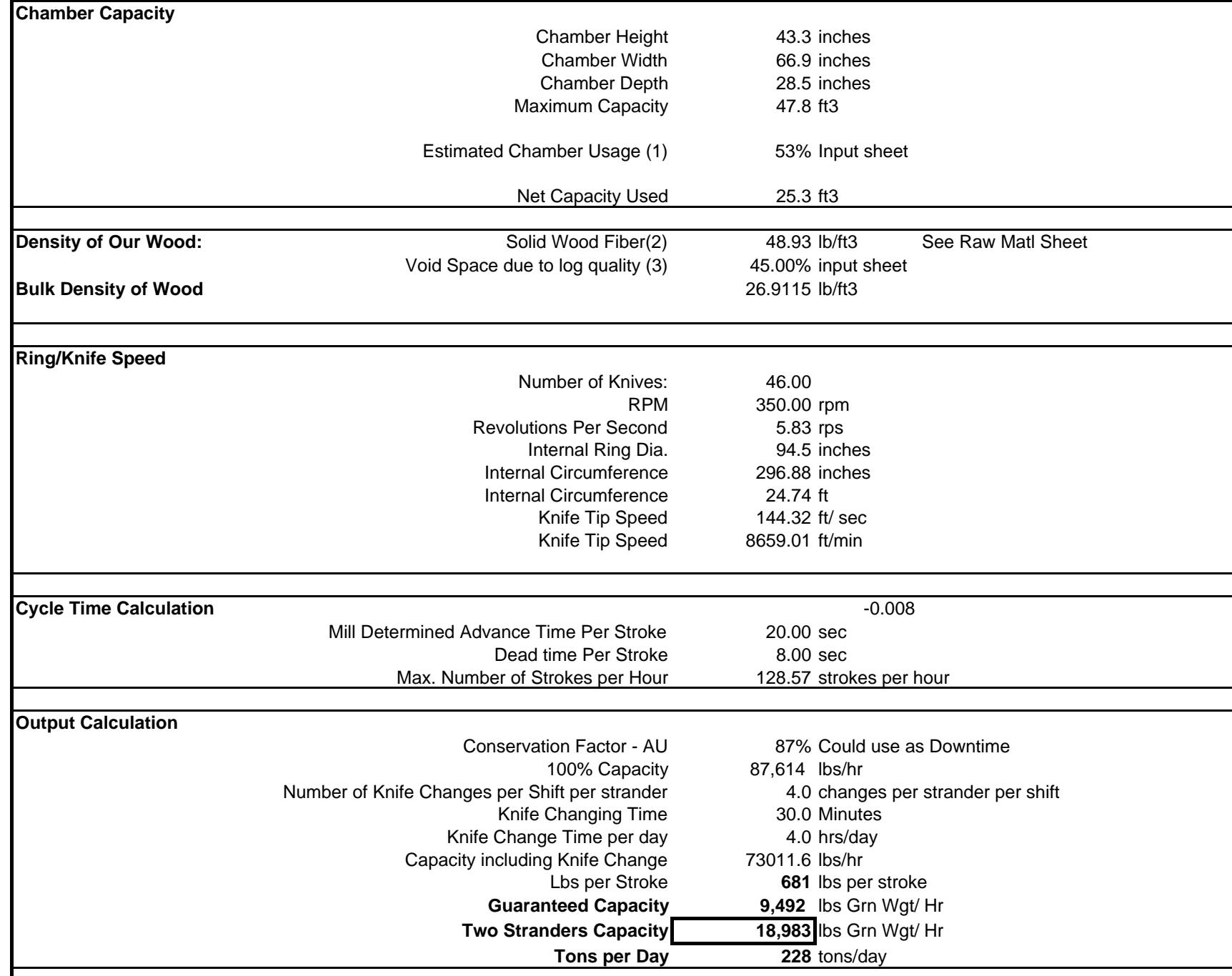

(1) The more the conveyor and chamber are filled the higher the Usage number

(2) Log Density in Grn Wgt per Grn Vol and is based on weighted average of species mix

(3) The straighter and smaller the logs the less void space and lower the number

\section{Increased Allowable Stroke Count Per Shift Per Strander}

Maximum Allowable Stroke Count

Total Possible Strokes in $12 \mathrm{hr}$ shift

Strokes in 12 hrs less changes

Lost Strokes Due to Changes

Lost Weight Due to Knife Changes

Average Weight Loss Per Change

Hours between changes

\# of changes required per shift

Time to Change

Average Strokes Per Shift per Strander

Total Strokes both Stranders

Short Fall in Max Possible

Minutes Lost (Strokes*Carriage Time)

Knife Changes Possible
1000.00 Set by Technical Based on Quality

1543 Based on Carriage speeds

1444

99

67,588 Assumes no other Down Time

43,807

7.8

1.54

30.0

778.00 IP21 Data

1556

765 strokes

357 minutes

11.89777778 


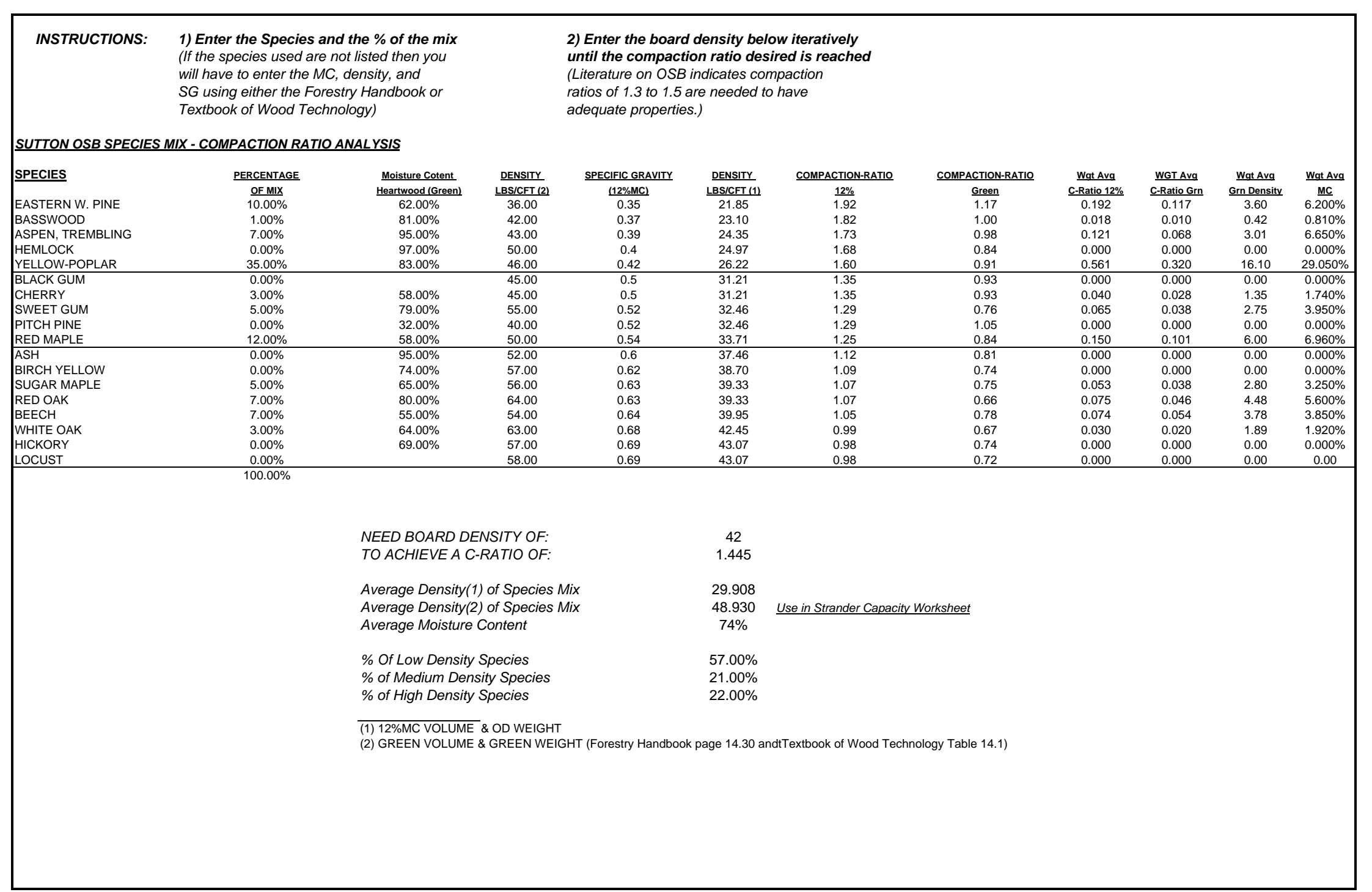

OSB Mill Model Species Mix - Compaction Ratio Analysis 


\begin{tabular}{|c|c|c|c|c|c|c|c|c|c|}
\hline \multicolumn{10}{|l|}{ Drying } \\
\hline & \multicolumn{2}{|c|}{ Needed Out of Dryer MC } & \multicolumn{2}{|c|}{ Formline MC Targets } & Green & \multicolumn{3}{|c|}{ Dryer Weights, Into Dryers } & \multirow[b]{2}{*}{ Per Dryer } \\
\hline & Surface & Core & Surface & Core & MC & Surface & Per Dryer & Core & \\
\hline \multicolumn{10}{|l|}{ Product } \\
\hline $7 / 16$ & $9.0 \%$ & $5.2 \%$ & $10.0 \%$ & $5.0 \%$ & $73.9 \%$ & 81,934 & 40,967 & 67,370 & 33,685 \\
\hline $1 / 2$ & $9.0 \%$ & $5.1 \%$ & $10.0 \%$ & $5.0 \%$ & $73.9 \%$ & 84,591 & 42,296 & 35,670 & 35,670 \\
\hline 19/32 Edge & $9.0 \%$ & $5.1 \%$ & $10.0 \%$ & $5.0 \%$ & $73.9 \%$ & 87,815 & 43,908 & 76,158 & 38,079 \\
\hline 23/32 Edge & $9.0 \%$ & $5.0 \%$ & $10.0 \%$ & $5.0 \%$ & $73.9 \%$ & 91,067 & 45,533 & 81,017 & 40,509 \\
\hline $23 / 32 \mathrm{EG}$ & $9.0 \%$ & $5.0 \%$ & $10.0 \%$ & $5.0 \%$ & $73.9 \%$ & 91,067 & 45,533 & 81,017 & 40,509 \\
\hline 3/4 P3 & $9.0 \%$ & $5.0 \%$ & $10.0 \%$ & $5.0 \%$ & $73.9 \%$ & 96,567 & 48,284 & 86,339 & 43,169 \\
\hline $7 / 8$ P3 & $9.0 \%$ & $5.0 \%$ & $10.0 \%$ & $5.0 \%$ & $73.9 \%$ & 97,719 & 48,860 & 88,285 & 44,142 \\
\hline \multicolumn{10}{|l|}{ Blending } \\
\hline & \multicolumn{3}{|c|}{ Wood Usage (Into Blenders) } & \multicolumn{6}{|c|}{ Resin Usage (lbs / msf3/8's) - Liquid Basis } \\
\hline & 1 & Per Blender & Core & Per Blender & Surface & Resin-Surface & Core & Resin-Core & Wax \\
\hline \multicolumn{10}{|l|}{ Product } \\
\hline $7 / 16$ & 47,127 & 23,563 & 42,605 & 21,303 & 13.00 & LPF & 5.875 & MDI & 12.37 \\
\hline $1 / 2$ & 48,655 & 24,327 & 44,541 & 22,271 & 12.91 & LPF & 5.907 & MDI & 12.36 \\
\hline 19/32 Edge & 50,509 & 25,255 & 46,890 & 23,445 & 12.82 & LPF & 5.953 & MDI & 12.36 \\
\hline 23/32 Edge & 52,379 & 26,190 & 49,260 & 24,630 & 12.74 & LPF & 5.992 & MDI & 12.36 \\
\hline 23/32 EG & 52,379 & 26,190 & 49,260 & 24,630 & 12.74 & LPF & 5.990 & MDI & 12.36 \\
\hline 3/4 P3 & 55,543 & 27,772 & 52,368 & 26,184 & 6.36 & MDI & 5.998 & MDI & 12.36 \\
\hline $7 / 8$ P3 & 56,206 & 28,103 & 53,277 & 26,638 & 5.98 & MDI & 5.998 & MDI & 11.65 \\
\hline
\end{tabular}




\section{OSB Mill MODEL}

This model may be used to determine: 1) the Production Mass Balance between the Dryers and Blenders

2)the effect of the process variables on recovery and production / hour

3 ) the effect of panel density on the cost of raw materials

PRODUCT

\section{SUMMARY}

(Don't Change Variables on
this worksheet use Input
Worksheet)

Uptime

Press Cycle

Press Loads Per Hour

Press Vol Per Hour

Press Vol Per Shift

Density

Formline Speed (theoretical)

Surface to Core Ratio
$100 \%$

$\begin{array}{cl}20 & \text { seconds per 16th } \\ 17.99 & \text { per hour } \\ 72,518 & \text { MSF per hour (3/8's) } \\ 870,216 & \text { MSF per shift (3/8's) }\end{array}$

$40.00 \quad$ pcf

$\begin{array}{ll}90.7 & \mathrm{fpm} \\ 460.6 & \mathrm{~mm} / \mathrm{sec} \\ 0.50 & \text { surface ratio }\end{array}$

Production Mass Balance

Blender Production I Blender (as measured by Blender Infeed Scales)

$\begin{array}{llll}\text { Surface } & \mathbf{2 3 , 5 6 3} & \text { pounds/hour across weigh belt when } \mathrm{MC}= & 10.0 \% \\ \text { Core } & \mathbf{2 3 , 2 3 0} & \text { pounds/hour across weigh belt when } \mathrm{MC}= & 5.0 \% \\ \text { Total Blended } & 93,587 & & \text { MC at Blender infeed }\end{array}$

Dryer Production by Furnish Type I Dryer (as measured by Dryer Infeed Nuclear Scales)

$\begin{array}{llll}\text { Surface } & \mathbf{4 0 , 9 6 7} & \text { pounds/hour across weigh belts when MC }= & 74 \% \\ \text { Core } & \mathbf{3 3 , 6 8 5} & \text { pounds/hour across weigh belts when MC }= & 74 \% \\ \text { Total Dried } & 149,304 & & \text { MC a }\end{array}$

Raw Material Costs

Surface LPF

Core $\quad \mathrm{MDI}$

Wax

Wood

TOTAL
Cost

$\$ 1.95$

$\$ 1.76$

$\$ 2.67$

$\$ 26.30$

$\$ 32.68$

per MSF

per MSF

per MSF

per MSF

per MSF
Weight (lbs per hr)

$\begin{array}{cl}943 & \mathrm{Ibs} / \mathrm{Hr} \text { (liquid) } \\ 426 & \mathrm{lbs} / \mathrm{Hr} \\ 897 & \mathrm{lbs} / \mathrm{Hr} \\ 87 & \text { Tons } / \mathrm{Hr} \\ 3.7 & \text { Trucks per Hr } \\ 89 & \text { Trucks per Day }\end{array}$

Recovery

0.761

MSF/Ton

\section{$74 \%$}

$74 \%$

MC at Dryer Infeed

Material Mass Balance

(Weight per Hour)

$\begin{array}{ll}\text { To Press (OD) } & \text { Surface Furnish } \\ & \text { Core Furnish } \\ & \text { FCOS and S-Trim }\end{array}$

From Blending (OD) Surface Furnish Core Furnish

To Blender (OD)

Surface Furnish Core Furnish

From Drying (OD)

To Dryer (GRN)
Surface Furnish

Core Furnish

System Five

Core Furnish

Surface Furnish

Core Furnish
Surface Furnish

$\begin{array}{cll}48,069 & \mathrm{lbs} / \mathrm{Hr} \text { (OD Wgt) } & \text { Wood + Additives } \\ 48,069 & \mathrm{lbs} / \mathrm{Hr} \text { (OD Wgt) } & \text { Wood + Additives } \\ 4,612 & \mathrm{lbs} / \mathrm{Hr} \text { (OD Wgt) } & \text { Wood + Additives } \\ 48,069 & \mathrm{lbs} / \mathrm{Hr} \text { (OD Wgt) } & \text { Wood + Additives } \\ 43,457 & \mathrm{lbs} / \mathrm{Hr} \text { (OD Wgt) } & \text { Wood + Additives } \\ 47,127 & \mathrm{lbs} / \mathrm{Hr} \text { (OD Wgt) } & \text { Wood NO Additives } \\ 42,605 & \mathrm{lbs} / \mathrm{Hr} \text { (OD Wgt) } & \text { Wood NO Additives } \\ 47,127 & \mathrm{lbs} / \mathrm{Hr} \text { (OD Wgt) } & \text { Wood } \\ 38,750 & \mathrm{Ibs} / \mathrm{Hr} \text { (OD Wgt) } & \text { Wood } \\ 3,856 & \mathrm{Ibs} / \mathrm{Hr} \text { (OD Wgt) } & \text { Wood } \\ 51,839 & \mathrm{Ibs} / \mathrm{Hr} \text { (MC Wgt) } & \text { Wood + Water } \\ 40,880 & \mathrm{lbs} / \mathrm{Hr} \text { (MC Wgt) } & \text { Wood + Water } \\ 81,934 & \mathrm{lbs} / \mathrm{Hr} \text { (Grn Wgt) } & \text { Wood } \\ 67,370 & \mathrm{lbs} / \mathrm{Hr} \text { (Grn Wgt) } & \text { Wood }\end{array}$

Per Machine

24,035

24,035

24,035

21,729

23,563

23,230

23,563

19,375

25,920

20,440

40,967

33,685 


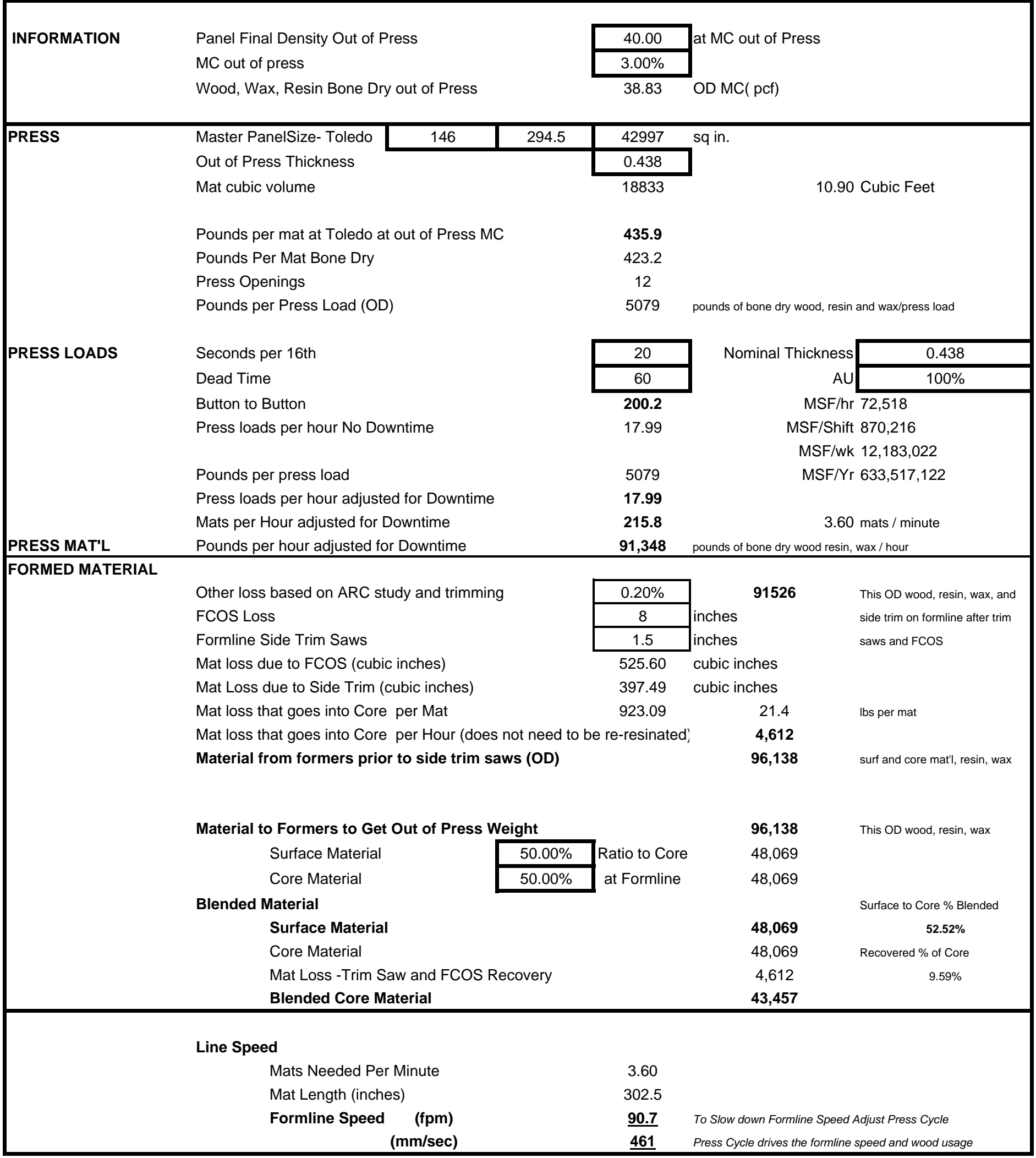


OSB Mill Model - Product (Page 3 of 4)

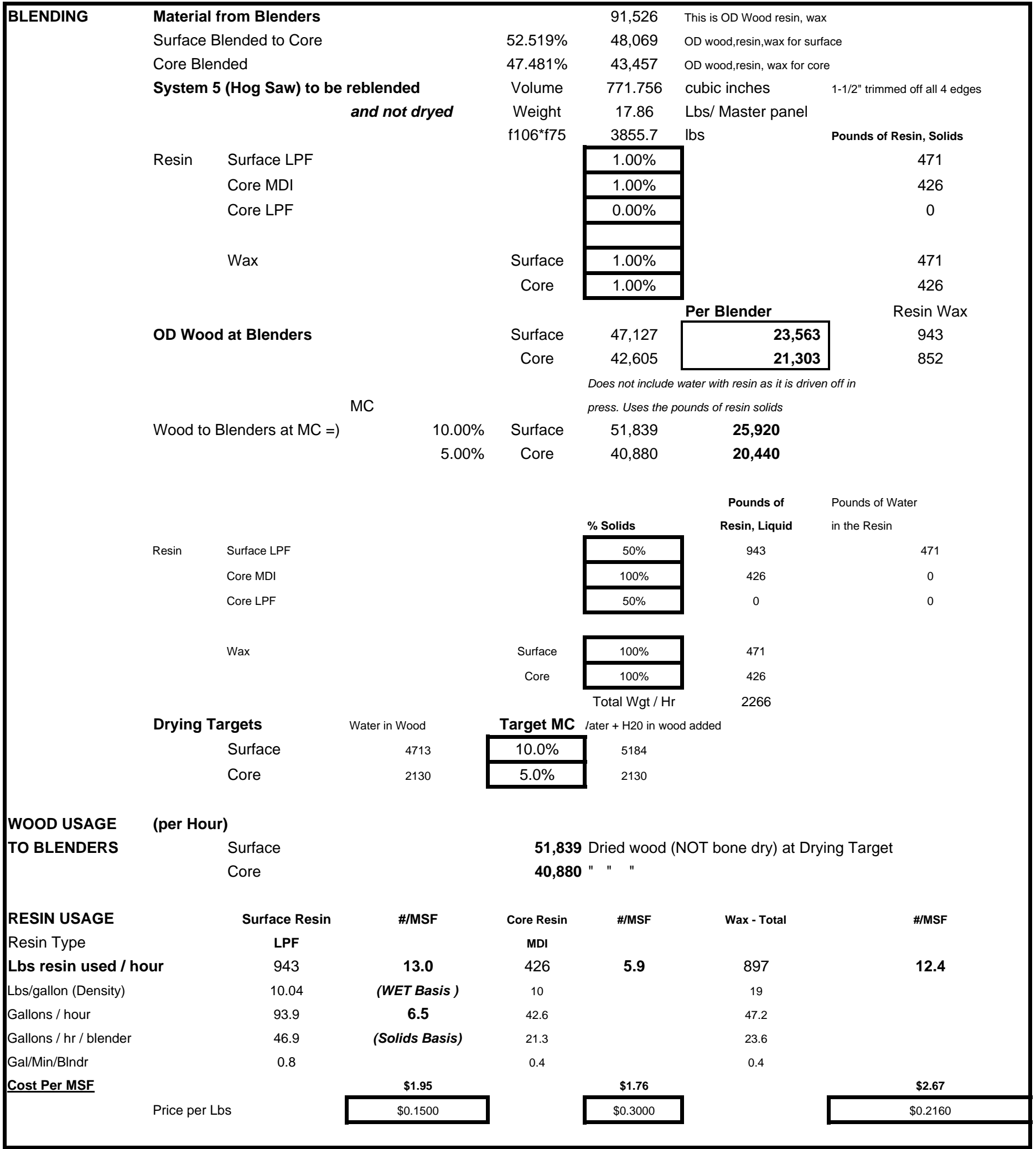


OSB Mill Model - Product (Page 4 of 4)

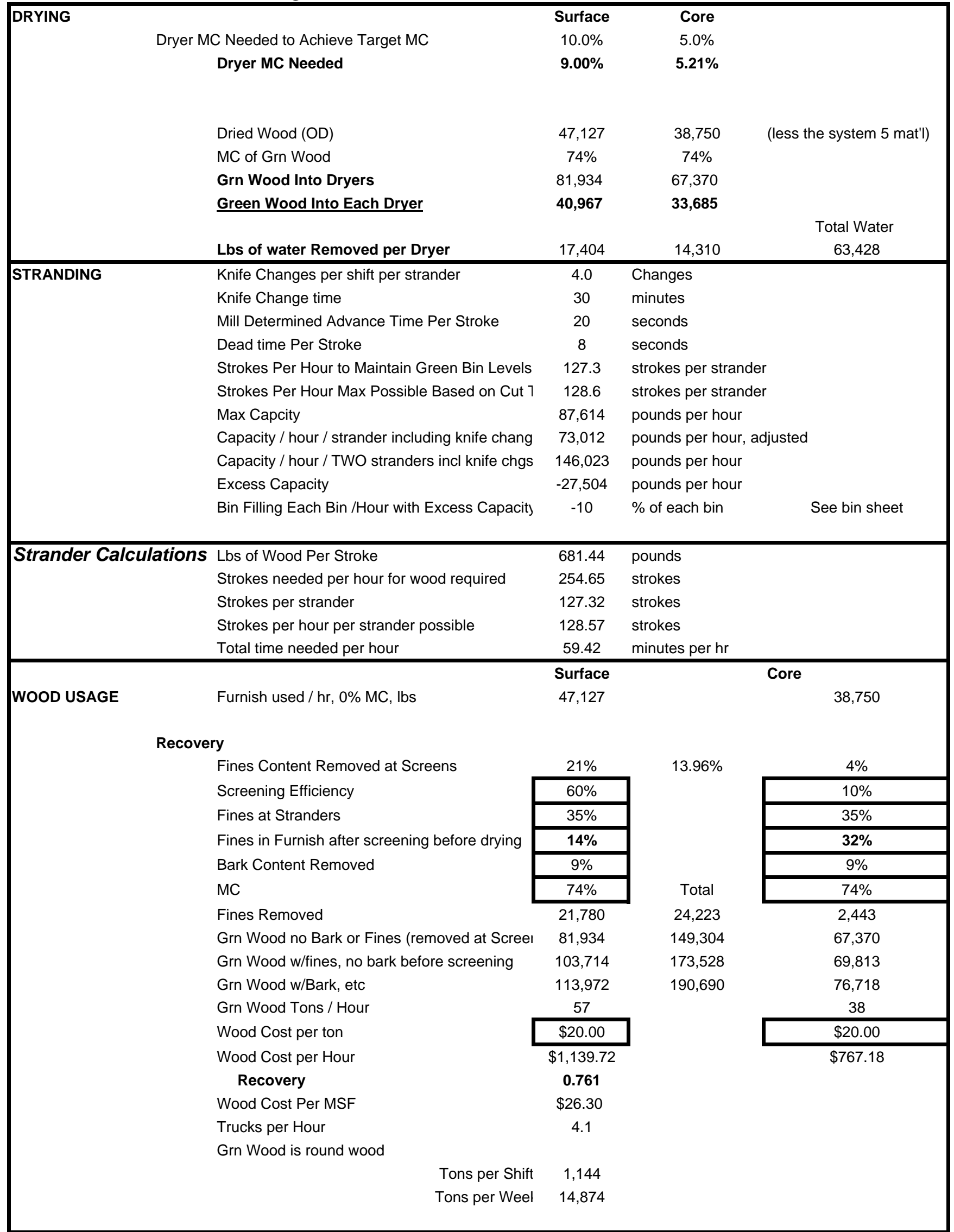




\section{APPENDIX B EFFECT OF AUTOCORRELATED DATA ON COMPOSITE PANEL PRODUCTION MONITORING AND CONTROL: A COMPARISON OF SPC TECHNIQUES (Published in For Prod J. 2002. Co-authored with R. B. Anderson)}

\section{B.1 ABSTRACT}

Traditional statistical process control (SPC) methodology is based on a fundamental assumption that the process data are independent. However, in continuous type processes, such as composite panel production, the process data are often highly autocorrelated. Under such conditions, traditional SPC techniques are not adequate and can lead to excessive searching for assignable or special causes that may not, in fact, be present in the process. A comparison of traditional and non-traditional SPC methodology for controlling the effect of autocorrelated processes in production monitoring and control are presented. The results of this comparison show that, for significantly autocorrelated data, the use of the autoregressive integrated moving average (ARIMA) control chart will provide a more consistent technique for detecting assignable or special causes in the continuous production processes.

\section{B.2 INTRODUCTION}

To improve product quality one needs to reduce product variability by keeping processes at or near their target value. This is accomplished by both process adjustment where one maintains the process as close as possible to the desired target value and process monitoring where one continually checks the stable state of the process looking

for assignable causes pointed to by using various control charts. Process adjustment is feedback control and used by control engineers. It is often referred to as engineering 
process control (EPC). Process monitoring, on the other hand is probability based, describing the probability of the point falling outside a control limit on say a Shewhart chart. It is used by statistical quality control practitioners and is referred to as statistical process control (SPC). EPC and SPC originated in different industries with SPC coming from the parts industries and EPC from the continuous process industries. SPC is focused on producing parts with the smallest possible variation around a fixed target, while EPC is focused on obtaining the highest possible mean values with the smallest variation. Box and Lucerno (1997) make the point that the lines of distinction between EPC and SPC are becoming blurred because of hybrid industries such as the computer chip manufacturer, of more conglomerate companies with both types of processes, and of the "quality revolution" where each industry is experimenting with each others control technology in efforts to improve quality. In fact they go on to state, "It is now generally accepted that activities of monitoring and adjustment should not be carried out separately, but should be performed simultaneously."

"Economic scarcity of wood fiber and the resulting raw material costs for manufacturers will be a driving force for change in the forest products industry in the $21^{\text {st }}$ Century. SPC...will help many forest products companies improve product quality, productivity, and competitive position.” (Young and Winistorfer 1999). The industry has used many of the SPC techniques to monitor the process and improve quality. This is changing now that many of the companies are conglomerates with both parts and continuous process in the same company and as pointed out above are now investigating how to use both SPC and EPC to improve product quality. This is evident in the APA, the engineered wood organization's approach to quality management 
systems with its two phases. The first is focused on SPC and product testing / adjustment, and the second is focused more on EPC and process monitoring / adjustment.

The SPC strategy for stabilizing and improving the process is to standardize procedures and to use hypothesis-generating devices (such as control charting, graphs, check sheets, Pareto charts, cause-effect diagrams, etc.) to track down and eliminate causes of trouble (Kume 1985). Control charting is designed to provide insights into the process that will lead to process improvements (Gilbert et al. 1997). Box and Luceno (1997) discuss the efficacy of several control charts for monitoring the process so that, the process is in a state of control. Where a state of control is defined as "the output of the process is varying in a stable manner about a fixed mean." They discuss the various control charts by stating "if the background disturbance is white noise, the Shewhart chart is efficient for looking for a spike, the moving average (MA) for looking for a rectangular bump, the Exponentially Weighted Moving Average (EWMA) for looking for an exponentially increasing signal (with discount factor the same as that for the EWMA statistic) and Cumulative Sum (Cusum) for looking for a step change." Investigating assignable causes or special causes is tedious and expensive. It usually makes economical sense to wait until "statistically significant" deviations occur from the stable system before investigating. Determining "statistical significance" is typically achieved by using process monitoring charts such as Shewhart charts, Moving Average, Exponentially Weighted Moving Average, and Cusum charts. The philosophy is "don't fix it when it ain't broke" - don't needlessly tamper with the process (Deming 1986). 
EPC or process adjustment strategy is focused on controlling the process when the process centerline differs from the target with control decisions typically based on each observation. Since the process is seldom on the center line, change is constant. SPC, on the other hand, waits until appropriate statistical significance is accumulated before a decision is made and therefore decision is not reached as quickly as with EPC. Process adjustment strategies are beyond the scope of this article but if one is interested in pursuing this area further Box and Luceno (1997) discuss this in greater detail.

With SPC being used more frequently by the forest products industry there is a tendency to use the tried and true traditional SPC techniques that grew out of discrete parts industries. The issue is the traditional SPC techniques will result in "detecting" more false alarms when one has a continuous parts process, which in turn will quickly create a distrust of SPC by practitioners when they cannot find the root cause for the "false alarm". It should also be pointed out that inappropriate use of any model, SPC or EPC type, can lead to inappropriate interpretations and actions. Therefore one should be warned that care should be taken in choosing a model to ensure it accurately describes the process of study. It should also be noted with SPC one important assumption is the process is stable. If it is not then it is inappropriate to use standard control limits and it may actually require some process adjustment strategy be used to reduce the variability.

The background portion is a short review of the traditional SPC techniques used with independent data and the SPC techniques used with autocorrelated data or dependent data. An example will demonstrate how autocorrelated data affect the interpretation of the various traditional control charts and increase the frequency with which false action signals are generated. Finally models will be developed from the 
example data set and demonstrate the false action signal effect based on the models. The "best" model will provide the most consistent control of the continuous production process.

\section{B.3 BACKGROUND}

\section{B.3.1 Traditional "Discrete Data" SPC}

The traditional SPC methodology, Shewhart, Cusum, and EWMA, have grown up in the discrete manufacturing industry, where typically one part at a time measurements are made. Traditional SPC methodology is based on a fundamental assumption that the process data are independent. A reasonable model for the observations from the process is:

$$
X_{t}=\mu+\varepsilon_{t}
$$

Where $\mu$ is the process mean and $\varepsilon_{\mathrm{t}}$ is a sequence of independently and identically distributed random variables. For example, if wooden furniture components are being made, the critical measurements on each successive batch of parts are naturally independent of each other. Two measurements are independent if the occurrence of one of the measurements gives no information about what the other measurement's value will be -- that is, the measurements have no influence on each other.

Unfortunately for many processes, particularly continuous processes, this may not be the case. The data may actually be dependent of each other or autocorrelated. The dependence arises from the inertia of the process that limits short-term variation (Gilbert et al. 1997). In continuous type processes such as composite panel processes OSB, MDF, and particleboard - the process data are typically autocorrelated. When a 
"process follows an adaptive model or when the process mean is a deterministic function - for example, a harmonic function or a linear or non-linear trend - the data will also be autocorrelated" (Zhang 1998). Also when the frequency of sampling is short the sequence of process observations will be autocorrelated (Montgomery and Mastrangelo 1991).

Under such conditions of autocorrelation, traditional SPC techniques are not adequate as pointed out by numerous authors. Young and Winistorfer (1999) discussed the effects autocorrelation has on the Shewhart chart which is to increase the number of false alarms and investigations for assignable causes. Johnson and Bagshaw (1974 and 1975) discussed the effects of autocorrelation on the performance of the Cusum chart. Harris and Ross (1991) discussed the impact on EWMA charts and pointed out the average and median run lengths of these charts are sensitive to the presence of autocorrelation. Montgomery and Mastrangelo (1991) stated the, "primary impact is to increase the frequency with which false action signals are generated; that is, the incontrol average run length is much shorter than advertised". They went on to say "even very low levels of serial correlation will produce dramatic disturbances in these control chart properties".

\section{B.3.2 Autocorrelated Data SPC}

To accommodate autocorrelated data, some additional SPC methodologies have been developed in recent years. These include, but are not limited to as more methodology is being developed each year:

1) Process Residual Charts Using Models and plotting the model residuals using the traditional SPC Charts, 
2) Adjusting Traditional SPC Chart Control Limits, and

3) One-Step-Ahead EWMA

\section{B.3.2.1 Process Residual Chart Using Models}

One approach is to use a process residual chart (Alwan and Roberts 1988). The procedure requires the process data be modeled using a time series model such as the autoregressive integrated moving average (ARIMA) model (see Appendix C or for a more detailed discussion see Box, et al. (1994)). Then assuming a true model, the process residuals are statistically independent and can be control charted using the traditional SPC charts. If a shift in the mean occurs, the identified model is no longer correct and the model error will be transferred to the residuals. A control chart applied to these residuals will ultimately detect the shift in the mean (Montgomery and Mastrangelo 1991). The traditional charts such as Shewhart individuals, Cusum, and EWMA charts can be applied to the residuals. Zhang (1998) elaborated on this technique.

Use of the residual chart has the advantage that it can be used even if the data are from a non-stationary process, that is a time series that wanders about with no fixed mean. When a residual chart is applied to a non-stationary process and gives a signal, one can only conclude the process has some system deviation because a non-stationary process has no constant mean and/or constant variance.

It should be noted the residual charts do not have the same properties as the traditional charts and the detection capability may be less. (Harris and Ross 1991, Longnecker and Ryan 1992, Wardell et al. 1994, and Zhang 1997) Besides the limitation on detection capability of the residual chart, another disadvantage is time 
series modeling is often awkward in the mill or SPC environment. With the increased availability of computers and statistical software such as Statgraphics ${ }^{\mathrm{TM}}$ (Manugistics 1999) this disadvantage is becoming less but is still awkward in the mill environment.

\section{B.3.2.2 Modifying Traditional SPC}

Another approach to accommodate autocorrelated data is to modify existing SPC charts by adjusting the control limits. Vasilopoulos and Stamboulis (1978) modified for some specific time series the $\mathrm{x}$-bar chart control limit. The authors acknowledged that the "computations become prohibitive..." and this approach is limited to sub-grouped data and x-bar charts. Gilbert et al. (1997) proposed adjusting individual control charts when the assumption the autocorrelation at lag 1 is significant by adjusting the $\mathrm{d}_{2}$ value. The value can be computed as:

$$
\mathrm{d}_{2}=2 \sqrt{\frac{1-r_{1}}{\pi}}
$$

The control limits are computed as:

$$
\begin{aligned}
& \bar{X} \pm 3\left(\overline{m R} / d_{2}\right) \\
& \text { where: } \quad r_{1} \text { is the autocorrelation at lag } 1 \\
& \frac{X}{m R} \text { is the process average }
\end{aligned}
$$

\section{B.3.2.3 One-Step-Ahead EWMA}

A third procedure for working with autocorrelated data is to plot one-step-ahead EWMA prediction errors on a control chart. Montgomery and Mastrangelo (1991) discuss this approach in some detail. They give an example of a process that can be modeled as ARIMA $(0,1,1)=\operatorname{IMA}(1,1)$ or integrated moving average model, say 


$$
X_{t}=X_{x-t}+\varepsilon_{t}-\theta \varepsilon_{t-1}
$$

The EWMA with $\lambda=1-\theta$ is the optimal one-step ahead forecast for this process. $\lambda$ is referred to as the damping factor. It is $1-\theta$ where $\theta$ is the discount or smoothing factor. A small discount factor, say 0.2 , will determine the forecast based on more current data, while a larger discount factor, say 0.8 , will determine the forecast based on more historical data. The damping factor to use is described by Box and Luceno (1997) on page 140 as minimizing the ratio MSE(adjusted) to MSE(unadjusted). They go on to state where the circumstances for "formal estimation of the damping factor is tedious, we can simply employ a compromise value, in the neighborhood of, say 0.2 to 0.4 ." That is, if $\hat{X}_{t+1}(t)$ is the forecast for the observation in period $t+1$ made at the end of period $t$, then

$$
\hat{X}_{t+1}(t)=Z_{t}
$$

The sequence of one-step-ahead prediction errors

$$
e_{t}=X_{t}-\hat{X}_{t}(t-1)
$$

are independently and identically distributed with mean zero and standard deviation $\sigma_{p}$ if the underlying process is really IMA $(1,1)$. Therefore control charts could be applied to these one-step-ahead prediction errors. For example, a control chart for individuals would have center line zero and upper and lower control limits at $\pm 3 \sigma_{p}$.

Montgomery and Mastrangelo (1991) went on to say, "suppose the process is not exactly ARIMA $(0,1,1)$ but is instead modeled by some other member of the ARIMA family. In general, if the observations from the process are:1) positively 
autocorrelated and 2) the process mean does not drift too quickly, the EWMA with an appropriate value for $\lambda$ will provide an excellent one-step-ahead predictor. Many processes follow a slow random walk and can be well represented by the EWMA."

\section{B.4 OBJECTIVE}

The objectives of this study were: (a) to demonstrate how autocorrelated data affect the interpretation of Shewhart, Moving Average, Cusum, and EWMA control charts; (b) to compare the use of time series models in the case of autocorrelated data with the models in (a); and (c) to develop a best model that provides the most consistent control of a production process. This will be accomplished by using an example of real continuous process data from an oriented strandboard furnish drying system.

\section{B.5 MATERIALS AND METHODS}

\section{Example - Oriented Strandboard Process}

Consider the following example of an oriented strandboard, continuous production process. Furnish is stranded from hardwood, pulpwood logs. Each strand is approximately 4 " long by 1 " wide by 0.028 " thick. The strands are dried to a target moisture content of $6.5 \%(\mathrm{MC}-\mathrm{OD})$ in a triple pass rotary dryer using outlet temperature control. An infrared meter is located on the dryer out-feed to automatically determine the furnish outlet moisture content. Operator interface is used to control the outlet moisture content by adjusting the outlet temperature and the in-feed weight. The resin and wax are applied to the strands in rotating blenders based on furnish weight corrected for strand moisture content. An infrared meter, located on the weigh belt feeding the blender, automatically determines the moisture content. The strands are then 
formed or oriented onto a moving screen as the screen passes under four forming stations. As the mat is formed, it travels over several in-line scales that monitor the amount of furnish being "laid-down". The scale values are used to automatically adjust the amount of material to lay down in subsequent forming stations. The forming system corrects or adjusts the weight depending on the amount of furnish moisture. Infrared meters located after two of the forming stations automatically determine the moisture content of the strands. These moisture content values are used in an automatic feedback loop to adjust the weight of material to laid-down.

Moisture content is a process variable that is very important in producing consistent quality OSB panels. Excessive variation in dryer furnish moisture contents increases the variation in panel weights, which have high correlation with many panel strength properties. By controlling the furnish moisture content the process variation is reduced.

The dryer furnish moisture contents were collected from a Wonderware ${ }^{\mathrm{TM}}$ process historian. The scan rate was once every 10 minutes over a 96 hour continuous time period. The data were reviewed and 74 observations were discarded due to the drying system being shut down with no wood flow. This reduced the total number of data points to 502 or $83 \mathrm{hrs}$.

\section{B.6 RESULTS AND DISCUSSION}

The data were analyzed to determine the severity of the autocorrelation. The actual data are plotted over time (Figure B.1). The plot shows considerable scatter or dispersion with the mean drifting over the time period of study. The drifting mean may indicates the EWMA One-Step-Ahead approach may not be acceptable as discussed by 
Montgomery and Mastrangelo (1991). But if one assumes the process is following as "slow random walk" then the EWMA model is acceptable (Montgomery and Mastrangelo 1991).

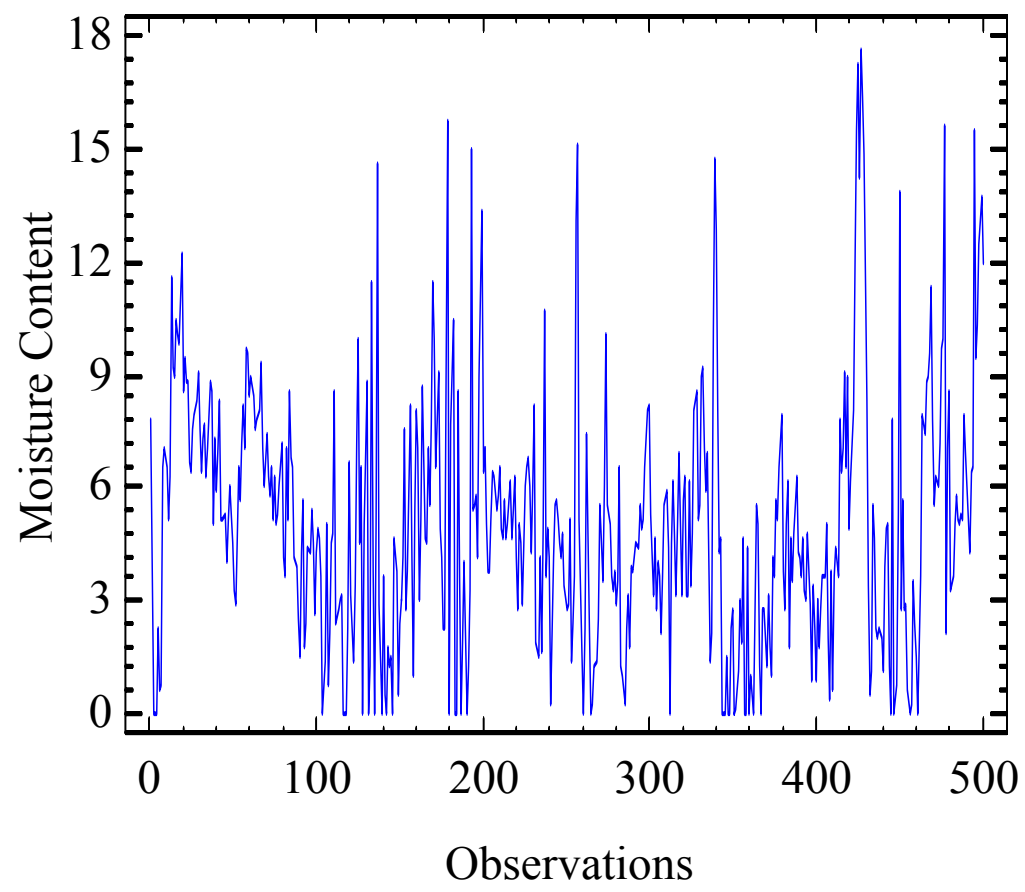

Figure B.1 Time series plot of moisture contents from a rotary, triple pass dryer.

The sample autocorrelation function was calculated where the sample autocorrelation at lag 0 is $r_{0}=1$, and $r_{0}, r_{1}, r_{2}, \ldots$ is the sample autocorrelation function. Where:

$$
r_{k}=\frac{C_{k}}{C_{0}}
$$




$$
c_{k}=\frac{1}{n} \sum\left(y_{t}-\bar{y}\right)\left(y_{t+k}-\bar{y}\right)
$$

The magnitude of the sample autocorrelation function is shown in Figure B.2. This Figure shows the data are significantly autocorrelated $(\alpha=.025)$ out to a lag of 7. It should be noted that while the authors were not considering adjusting the control limits to the traditional control charts as part of this study, the amount of autocorrelation would indicate the adjustments to the individuals control chart would not apply as discussed by Gilbert et al. (1997) and discussed in the background section above in this paper as the corrections suggested were for adjusting a process with a significant autocorrelation at a lag of only 1 .

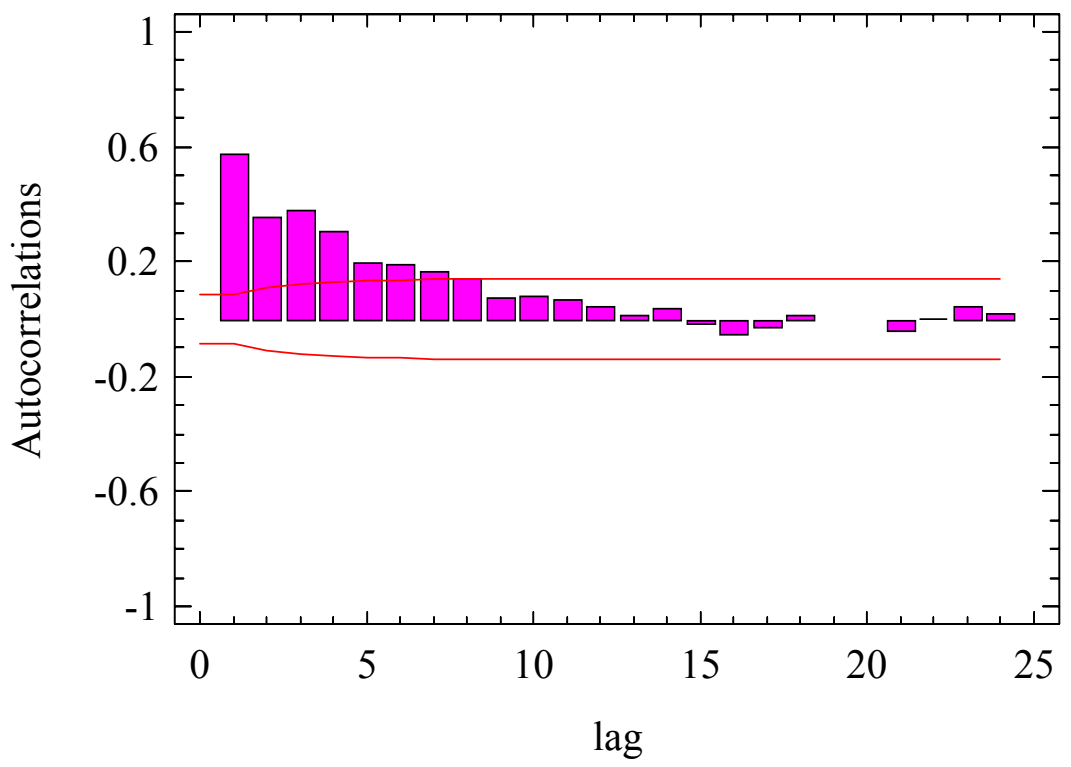

Figure B.2 Autocorrelation for moisture content time series data with a $95 \%$ confidence interval 
To determine the impact of autocorrelation the data were plotted using the Shewhart individuals chart, the moving average chart, the CUSUM chart and the EWMA chart. These are shown in Figures B.3 to B.6. The number of points out of control or special causes varies considerably by chart type. The Shewhart individuals chart indicated 27 signals for out of control conditions or special causes. The moving average chart had considerable more signals with 146 . The control limits for the moving average chart were much tighter than the individuals chart. Table B.1 shows the results for each of the four charts.

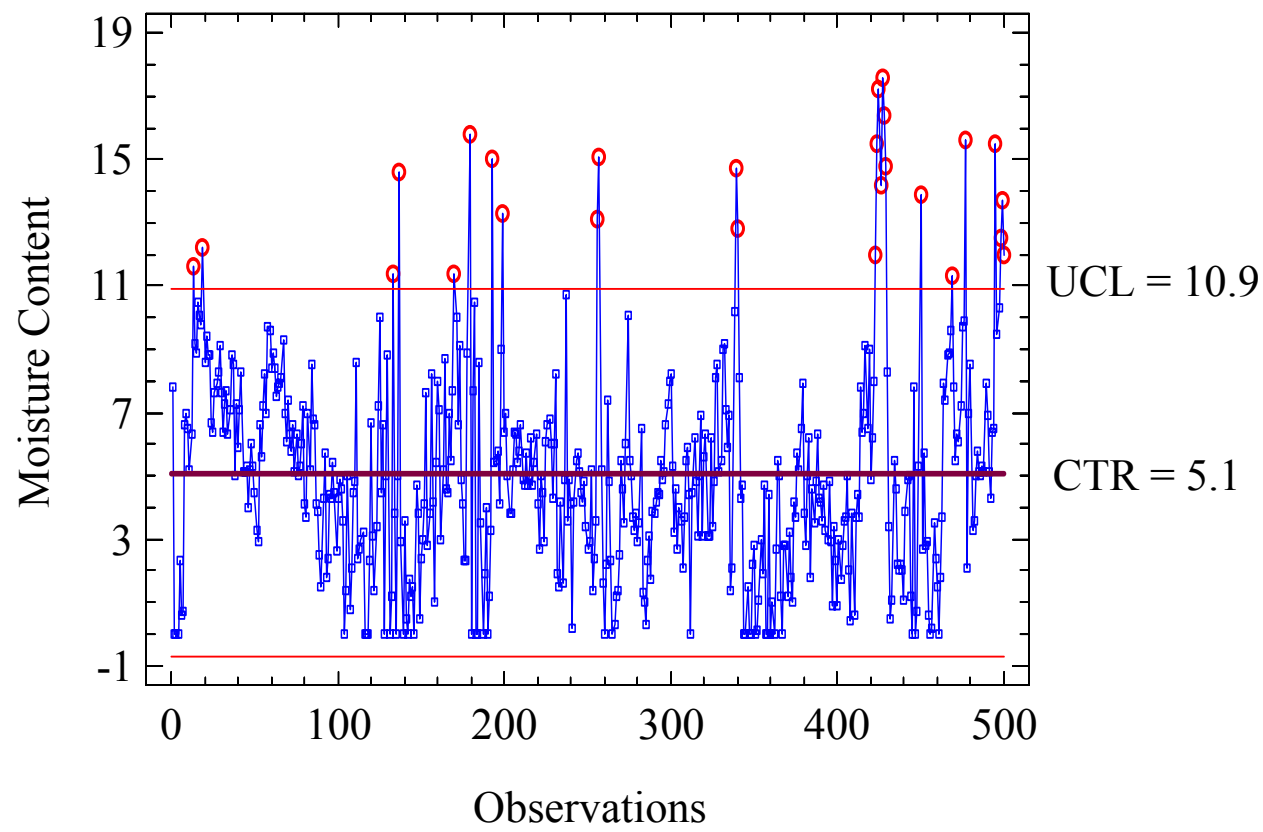

Figure B.3 Shewhart Individuals control chart for moisture contents from a rotary, triple pass dryer. 


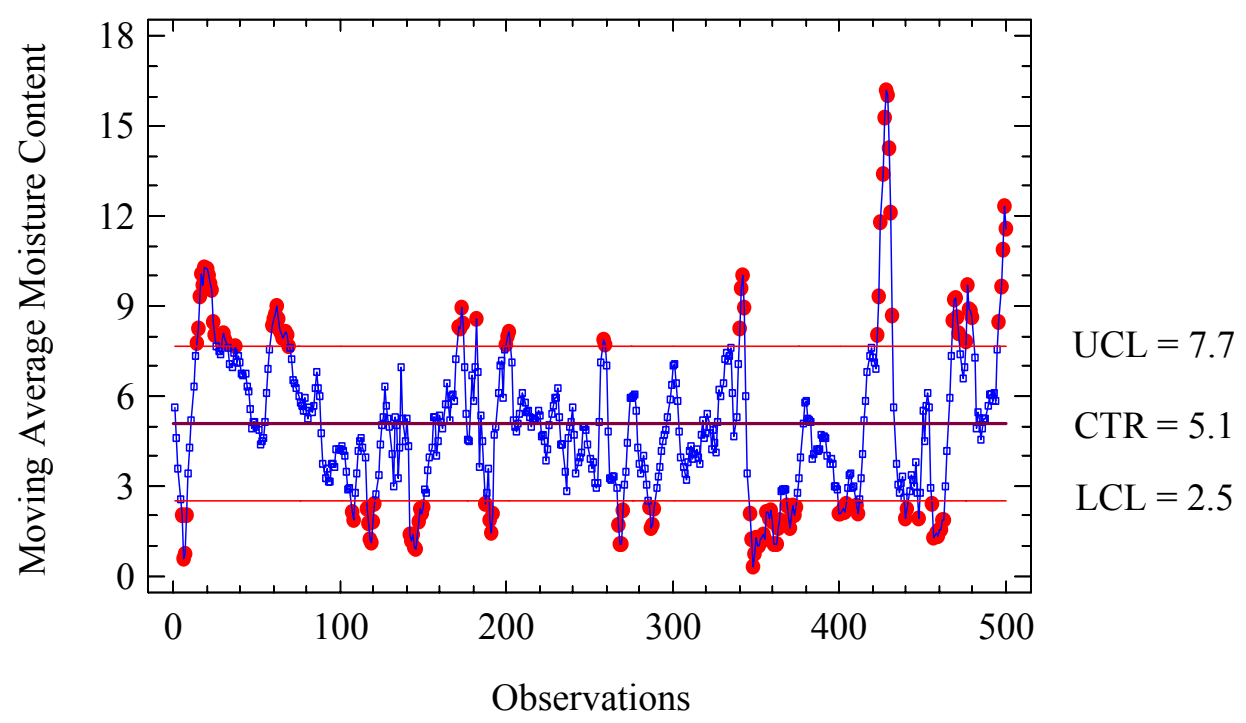

Figure B.4 Moving average control chart for moisture contents from a rotary, triple pass dryer.

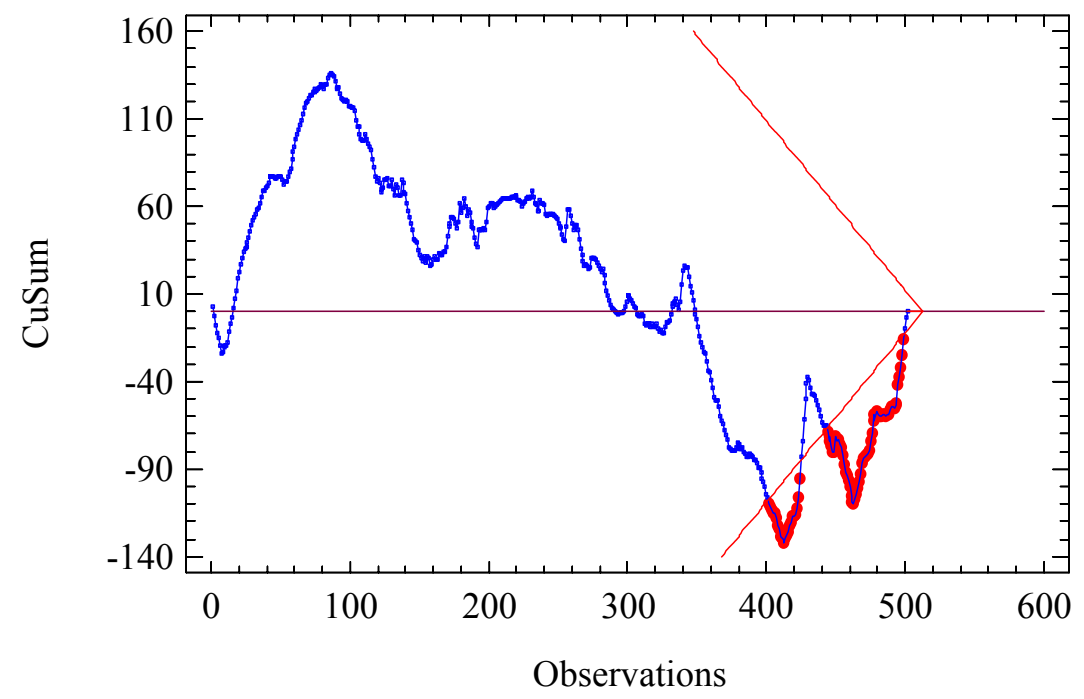

Figure B.5 Cusum control chart for moisture contents from a rotary, triple pass dryer. 


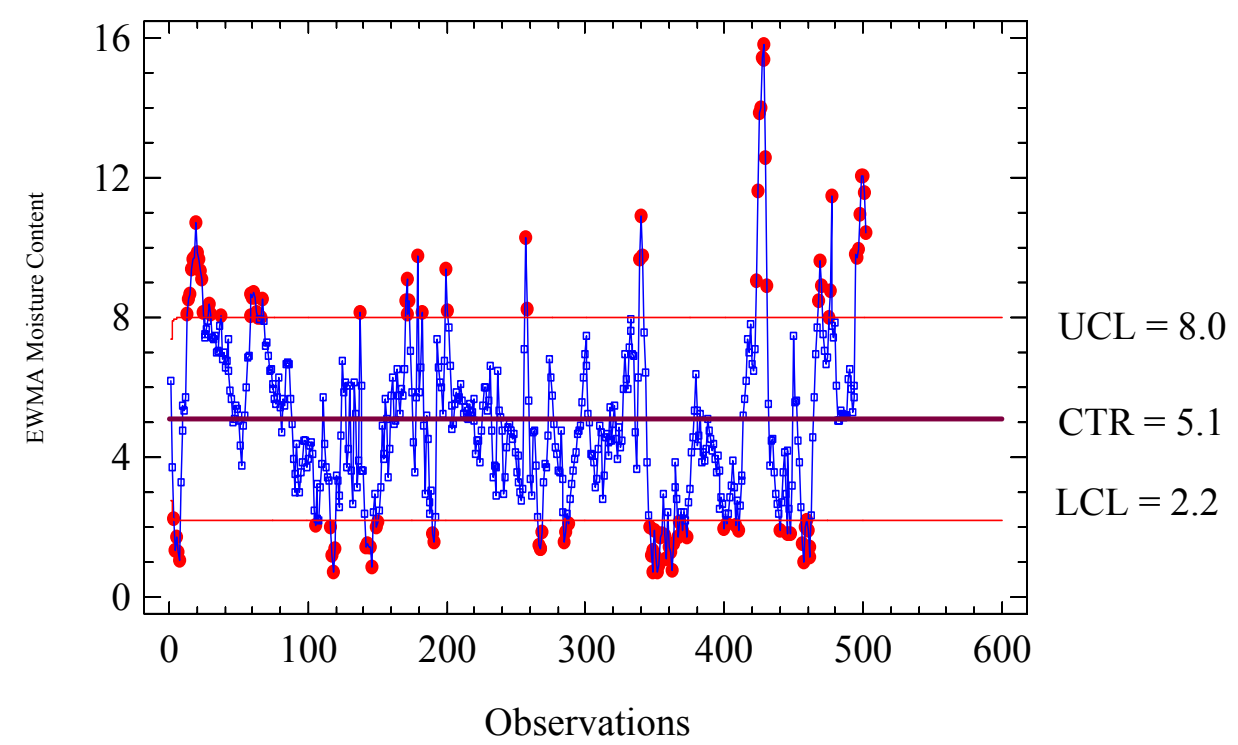

Figure B.6 EWMA control chart for moisture contents from a rotary, triple pass dryer.

Table B.1 Summary of control charts investigated.

\begin{tabular}{lccc}
\hline Chart & $\begin{array}{c}\text { Number of } \\
\text { Signals or } \\
\text { Observations out } \\
\text { of Control }\end{array}$ & $\begin{array}{c}\text { Lower } \\
\text { Control } \\
\text { Limit }\end{array}$ & $\begin{array}{c}\text { Upper } \\
\text { Control } \\
\text { Limit }\end{array}$ \\
\hline Shewhart - Individuals & 27 & N/A & 11.2 \\
Moving Average, Order 5 & 146 & 2.5 & 7.9 \\
EWMA with $\lambda=0.4$ & 111 & 2.1 & 8.2 \\
Cusum & 71 & & \\
ARIMA (1,0,2) Residuals & 11 & 2.6 & 17.9 \\
ARIMA (1,0,2) Individuals & 8 & Moving & Limits \\
\hline \hline
\end{tabular}

Depending on which chart an operator was using, incorrect adjustments to the process could result due to the number of false out of control signals. Deming (1986) in 
his funnel experiments showed the consequences of over adjusting a process, and he called it "tampering" which results in increased variation and poorer product quality.

The data were then evaluated using the ARIMA model. The best model was determined using the statistical software package, Statgraphics ${ }^{\mathrm{TM}}$ (Manugistics 1991). The ARIMA $(1,0,2)$ was chosen as it had the minimum mean square error of 5 models tried (Table B.2). Many more ARIMA models could have been tested using the Statgraphics ${ }^{\mathrm{TM}}$ software but they were not chosen to keep the model parsimonious thus eliminating many of the higher order models. Other models were investigated but they did not have the minimum mean square error values found for the 5 models shown in Table B.2.

Table B.2 Models Used to Approximate the Rotary Dryer Moisture Content Data

\begin{tabular}{ll}
\hline \hline Models & Mean Square Error \\
\hline Random Walk & 9.67 \\
MA Order 5 & 9.17 \\
ARIMA $(2,2,2)$ & 8.15 \\
ARIMA $(0,2,2)$ & 11.32 \\
ARIMA $(1,0,2)$ & 7.31 \\
\hline \hline
\end{tabular}

Figure B.7 shows the ARIMA model plotted with the actual data. The actual or raw data, shown as small squares, are the actual moisture contents from the process and are uncorrected. The data has a lot of scatter with some extreme points indicating the process is not in control. The ARIMA model, shown by the line, is an attempt to model the process. If the model is assumed to adequately represent the process by a low mean 
square error, then the residuals, model less actual, can identify when the process is out of control.

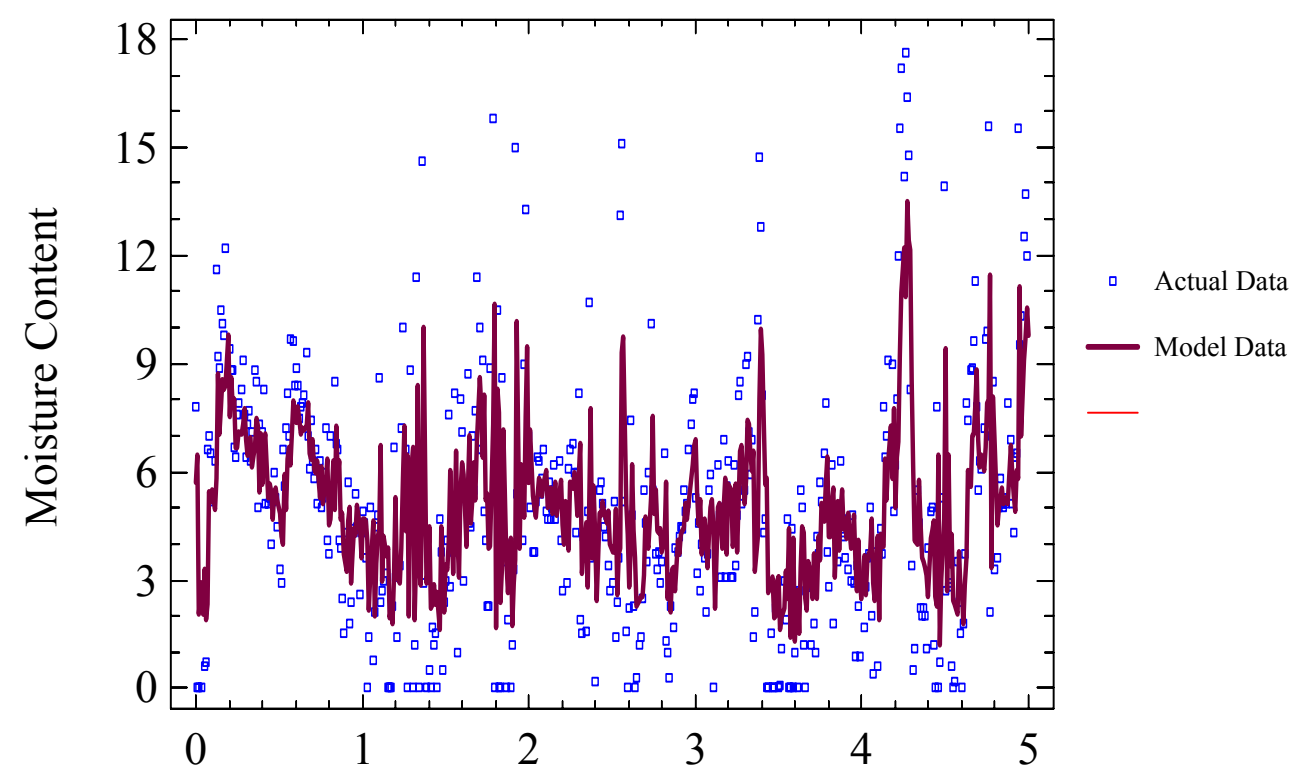

Figure B.7 ARIMA $(1,0,2)$ model with actual moisture contents from a rotary, triple pass dryer.

The residuals are then plotted using the Shewhart individuals chart. The result is only 11 signals indicating out of control situations (see Figure B.8.). The number of signals are considerably less than that shown by the four charts discussed earlier (see Table B.1). This would mean operational personnel would not make as many adjustments resulting in less tampering and better product quality. In addition it would mean an organization can be more focused on discovering the root cause(s) for the 
fewer "real" special causes and not chase after multiple phantom causes that can occur with tampering. Organizations that chase the phantoms quickly tire and may drop the idea of using control charts as it becomes too hard to discern a true cause for the phantom problem caused in many cases by tampering.

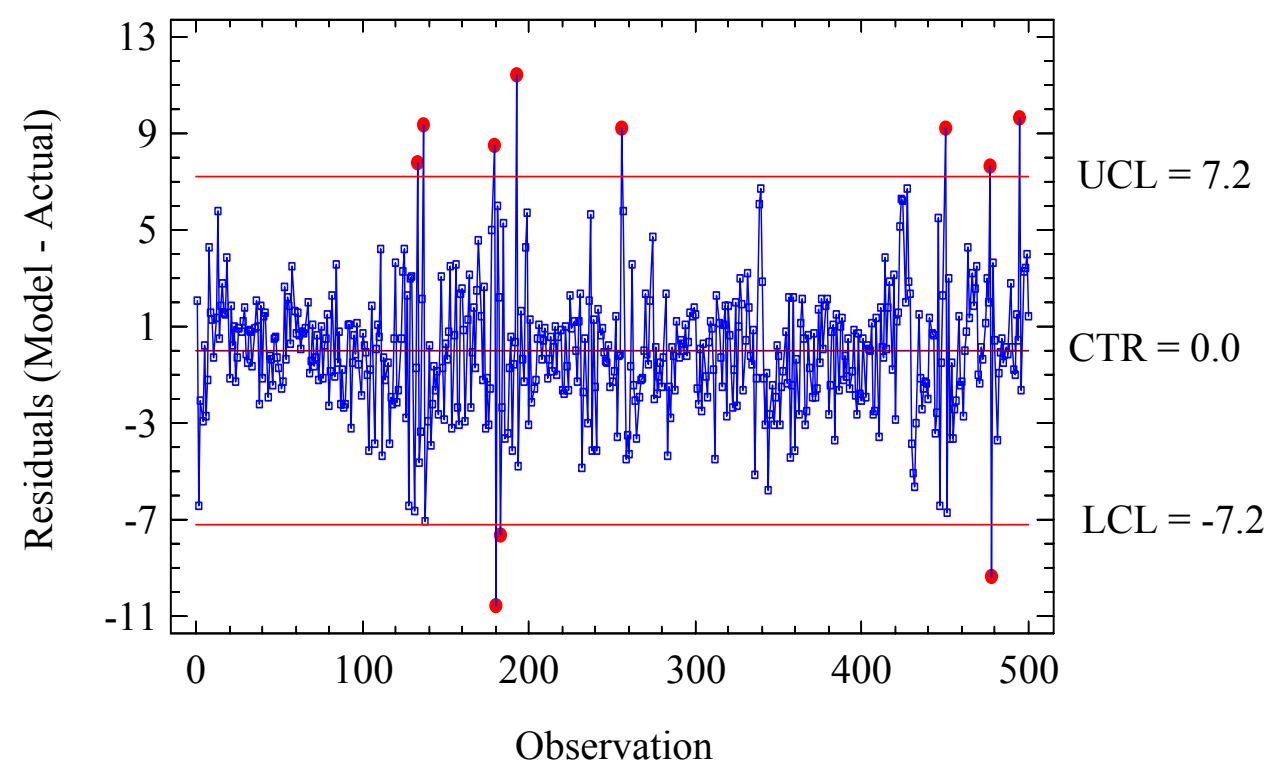

Figure B.8 Shewhart Individuals control chart of residuals from the ARIMA $(1,0,2)$ modeled moisture content data.

A stand alone ARIMA individuals charting program also part of Statgraphics ${ }^{\mathrm{TM}}$ was tried to see if a more "user friendly" approach could be derived. This chart uses moisture contents and not residuals. The operational personnel can typically identify or understand moisture content values much easier than residual values. The chart does however use a moving centerline and limits are based on one-ahead forecast errors. Montgomery and Mastrangelo (1991) discussed this approach in more detail than can be 
covered by this paper. Forecast errors are calculated by subtracting the estimate of the predicted value at time $\mathrm{t}+1$ from the actual value at $\mathrm{t}+1$. By already knowing the model ARIMA $(1,0,2)$, the chart was quickly developed. It showed only 8 signals (see Figure B.9), one less signal than the residual approach used above.

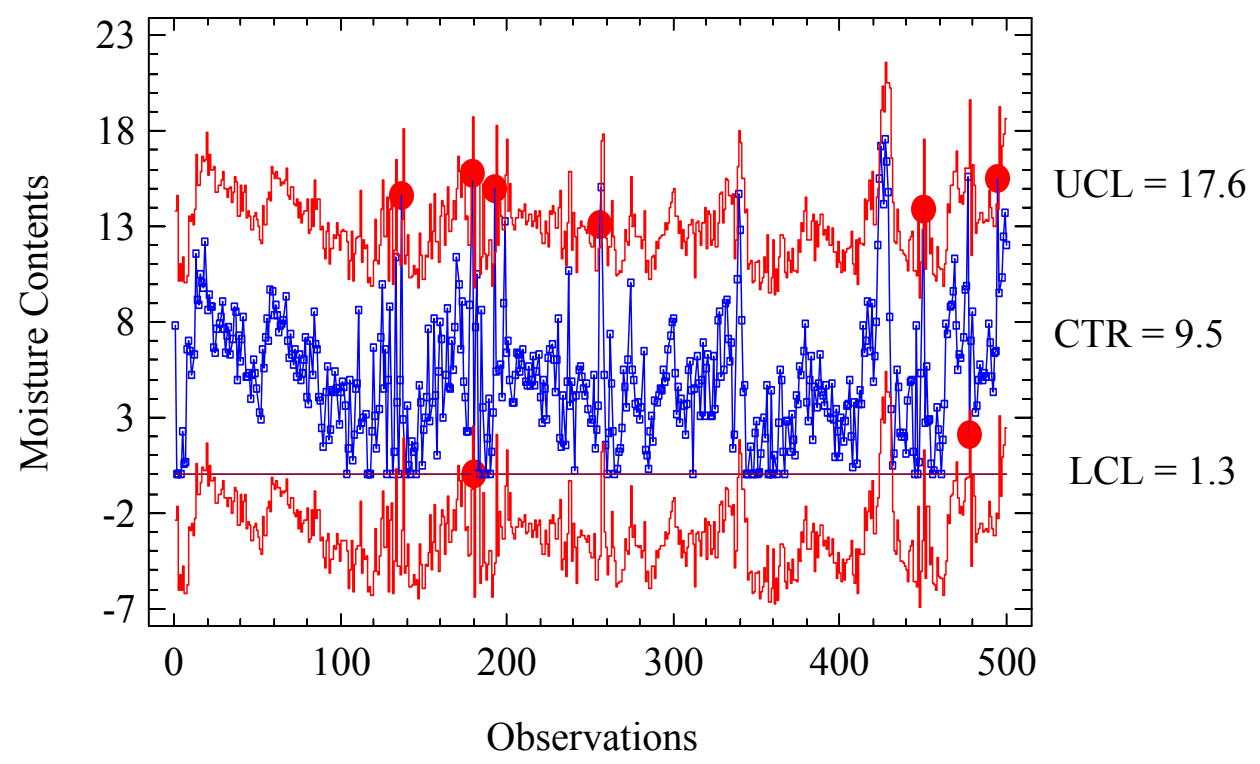

Figure B.9 ARIMA $(1,0,2)$ individuals control with limits based on the one-ahead forecast errors.

Using the ARIMA individuals chart the simplified approach was then tested on the next 12 hours of data from the drying process. The results are shown in Figure B.10. The two out of control signals were for 1) a rapid increase in the furnish feed rate (not a stepped gradual increase), and 2) a spark detect with a water mist to quench the spark. The data were also charted using the Shewhart individuals chart and 4 out of 
control signals resulted. If an operator used the individual chart, two additional adjustments to the process would have been made or tampering.

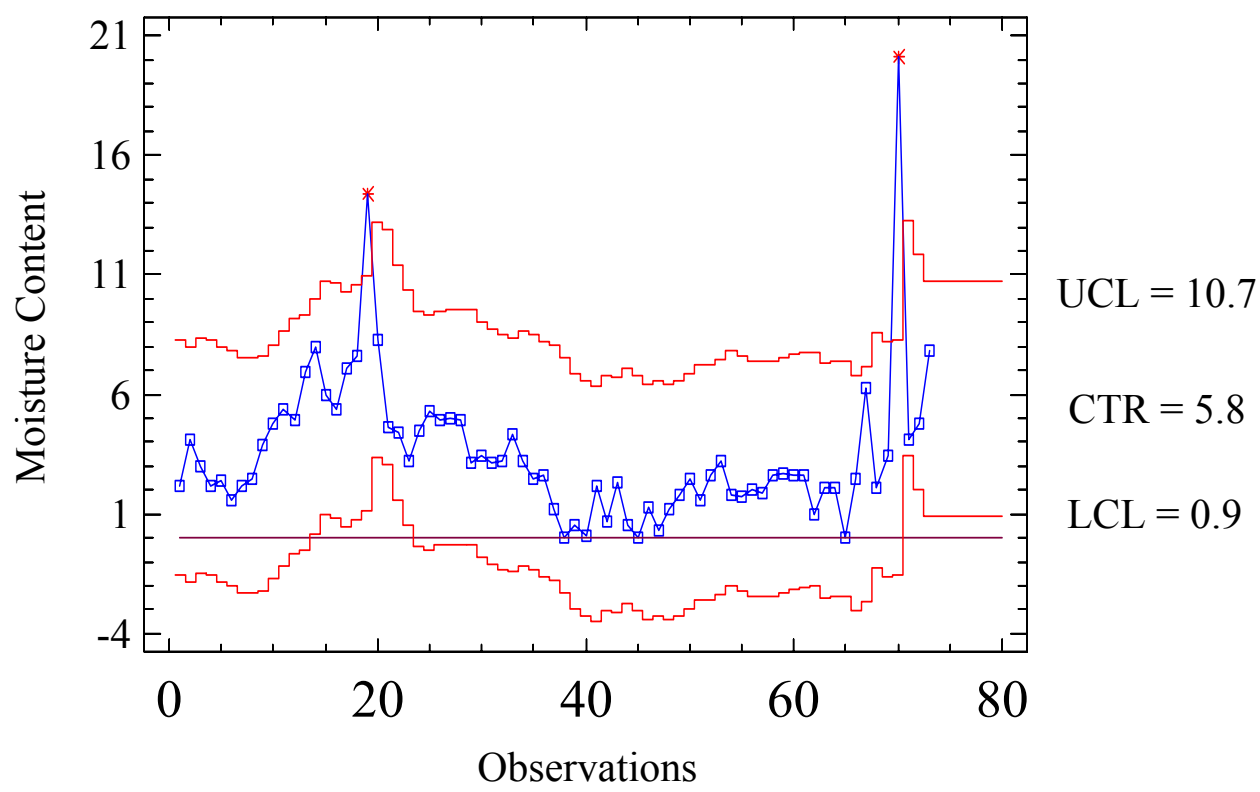

Figure B.10 ARIMA $(1,0,2)$ individuals control chart for new moisture content data from a rotary, triple pass dryer.

\section{B.7 SUMMARY AND CONCLUSIONS}

In continuous type processes, such as composite panel production, the process data are often highly autocorrelated. Under such conditions, traditional SPC techniques are not adequate and can lead to excessive searching for assignable or special causes that may not, in fact, be present in the process. The comparison of traditional and nontraditional SPC methodology show that traditional SPC techniques produce significantly more out of control signals than the ARIMA model developed for the 
furnish moisture contents from the continuous drying operation. The results of this comparison show that, for significantly autocorrelated data, the use of the ARIMA control chart will provide a more consistent technique for detecting assignable or special causes in the continuous production processes. 


\section{APPENDIX C - ARIMA MODELS}

ARIMA is an important class of stochastic models for describing time series. It provides a range of models, stationary and non-stationary ${ }^{2}$, that represent many of the time series found in practice (Box and Luceno 1997 and Box et al. 1994).

There are three general classes of the ARIMA model: Autoregressive (AR), Moving Average (MA), and Intergrated (I) and various combinations of the three. One class of ARIMA models is the autoregressive (AR) model, the current value is expressed as a finite, linear aggregate of previous values of the process and a shock $a_{t}$ or white noise. We designate the values of a process at equally spaced times $t, t-1, t-2 \ldots$

by $Z_{t}, Z_{t-1}, Z_{t-2} \ldots$. Also let $\tilde{Z}_{t}, \tilde{Z}_{t-1}, \tilde{Z}_{t-2}, \ldots$ be deviations from $\mu$ for example:

$\tilde{Z}_{t}=Z_{t}-\mu$. The autoregressive (AR) process of order $\mathrm{p}$ is represented by:

\footnotetext{
2 "The property of stationarity implies that the generated time series varies in a stable manner about the fixed mean. It can make excursions from this mean, but it will always return." (Box and Luceno 1997) They continue by stating, "In practice, many processes, if they were not appropriately controlled, would permanently drift away from the targetpossibly with disastrous results." A time series that drifts away from the target or wanders about with not fixed mean has the property of non-stationarity (Box and Luceno 1997). Control systems, i.e. PID loop control as applied to dryer control or formline weight control are set up to correct for deviations from target and adjust the process back to target thus keeping the process from permanently drifting away.
} 


$$
\tilde{Z}_{t}=\phi_{1} \tilde{Z}_{t-1}+\phi_{2} \tilde{Z}_{t-2}+\phi_{3} \tilde{Z}_{t-3}+\ldots+\phi_{p} \tilde{Z}_{t-p}+a_{t}(\mathrm{C}-1)
$$

The variable $\mathrm{z}$ is regressed on previous values of itself; hence the term autoregressive. The autoregressive model expresses the deviation of the process as a finite weighted sum of $p$ previous deviations of the process, plus a random shock $a_{t}$ (Box et al. 1994).

Equivalently it expresses $\tilde{Z}_{t}$ as an infinite weighted sum of a's.

Another class of the ARIMA model is the finite moving average (MA). Here we make $\tilde{Z}_{t}$ linearly dependent on a finite number $q$ of previous $a$ 's. Thus

$$
\tilde{Z}_{t}=a_{t}-\theta_{1} a_{t-1}-\theta_{2} a_{t-2}-\ldots-\theta_{q} a_{t-q}
$$

is called a moving average (MA) process of order $q$ (Box et al. 1994).

A third class of ARIMA models is a combination of both the AR and the MA in the same model that achieves greater flexibility in fitting time series. In practice, adequate representation of stationary time series can be obtained with autoregressive (AR), moving average (MA), or mixed models (ARMA), in which $p$ and $q$ are not greater than 2 and are often less than 2 (Box et al. 1994). In the ARMA model, the series forecast is expressed as a function of both previous values of the series and previous errors from forecasting (Manugistics 1999).

A fourth class of the ARIMA Models is the integrated (I) or difference model It is used because many series actually encountered in industry are non-stationary (see footnote 1). Although the general level about which the fluctuations are occurring may be different at different times, the broad behavior of the series, when differences in level are allowed for, may be similar. This homogenous, non-stationary behavior can be 
represented by a model that calls for the $d$ th difference of the process to be stationary. This is the integrated or difference model. In practice $d$ is usually 0,1 , or at most 2 .

Finally combining all three - the autoregressive (AR), the integrated (I), and the moving average (MA) - one obtains what Box, et. al. (1994) call the autoregressive integrated moving average (ARIMA) process of order (p,d,q). It is defined by

$$
w_{t}=\phi_{1} w_{t-1}+\ldots+\phi_{p} w_{t-p}+a_{t}-\theta_{1} a_{t-1}-\ldots-\theta_{q} a_{t-q}
$$

with $w_{t}=\nabla^{d} z_{t}$ and where $\nabla$ is the backward difference operator ${ }^{3}$.

\footnotetext{
${ }^{3} \nabla$ is defined as the difference between observations such that $z_{t}-z_{t-1}$ for a backward difference operator $\nabla_{z_{t}}$
} 


\title{
CURRICULUM VITAE
}

\section{JOHN ROGER NOFFSINGER}

\section{E Shannon Road Bridgeport, WV 26330·304-842-4394·noffsij@verizon.net}

\section{Education}

West Virginia University, Morgantown, WV

Ph.D. in Forest Resources, expected May 2004

Dissertation: “ Modeling Oriented Strandboard Process and the Continuous Rotary Drying Process for

Oriented Strandboard"

Chairman: Dr. Bruce Anderson

University of North Florida, Jacksonville, FL

Master of Business Administration, May, 1984

The Pennsylvania University, University Park, PA

Master of Science in Forest Resources, March, 1976

Thesis: "Utilization of Black Cherry in Extruded Particleboard"

Advisor: Dr. Paul Kersavage

West Virginia University, Morgantown, WV

Bachelor of Science in Wood Science, December 1969

\section{Summary of Qualifications}

Research and Development / Product Development /

Process Improvement

Quality Management - Certified Quality Auditor

Operations Management

Strategic Planning

Internal Process Consultant

\author{
Statistical Analysis - MDVA and DOE \\ Statistical Process Control - Certified Quality \\ Engineer \\ Financial Analysis \\ Teambuilding / Facilitation \\ Technical Management
}

\section{Professional Experience}

\section{5 - Present Weyerhaeuser Company, Federal Way, Washington}

1995 - Present Technical Manager, Sutton, West Virginia Led the OSB organization in the implementation of the Quality Management System with numerous projects to improve quality, reduce cost, and increase productivity. Developed new products including Performance Plus Panel® with ICC ER 6190 design values. Edge Gold and Structurwood GOLD. Managed product quality assurance. Coordinated with sales and customers. Guided the effort to improve product quality through continuous improvement task teams. Strand thickness variation was reduced by over 19\%. Resin distribution was improved through several blending changes that in turn permitted LPF resin addition rates to be reduced by $10 \%$. Coordinated pressing improvements using the Press Monitor program such that cycle times were reduced and productivity increased by $9 \%$. Core furnish was modified with the introduction of particles which resulted in better machine profiling and increased recovery by over $4 \%$. Spearheaded the effort to improve drying control through the use of "real-time" control charting. Recently implemented a Cause-for-Loss program tracking external and internal product failures along with the corrective actions taken to eliminate or minimize a re-occurrence. Lead the effort in running "all PMDI". 
1985 - 1995 Champion International Corporation, Stamford Connecticut

1988 - $1995 \quad$ Lumber Operations Manager, Abbeville, Alabama. General manager of wood products production facility and leader in the transformation to non-traditional organization using TQM and CDQ. Led the organization in Kaizen/Total Quality Management effort. Results were numerous projects with emphasis on safety, quality, production and cost. Reduced LWDIR and workers compensations costs to less than \$5,000 in 1991. Implemented "safewalks" with team leaders and team members to raise awareness about safe and unsafe acts and conditions. Improved the lumber drying, control process, $\Delta \mathrm{T}$, to reduce drying related degrade to less than $1 \%$ which resulted in a savings of $\$ 300,000$ per year. Reduced sawing target sizes through sawing process control thus increasing recovery and reducing material costs. Increased employee involvement in problem solving and decision making process. Implemented job family concept, job certification, and pay for skill which increased job satisfaction and reduced turn over to less than $8 \%$ and absenteeism to less than $2.4 \%$. Improved production scheduling and forecasting which increased on time shipments to $92 \%$.

1987-1988 Process Facilitator / Internal Consultant, Camden, Texas Coordinated the Participative Management process for the Eastern Manufacturing Operations.

1985 - 1987 Manager of Administration, Camden Texas Reported directly to the VP of Operations, Eastern Manufacturing. Managed administrative functions including: training, safety, salary administration, and organizational development for Eastern Manufacturing - 4 plywood, 3 studmills, and 5 dimension lumber mills.

1975 - 1985 St Regis Paper Company, New York, New York

1981-1985 Senior Project Engineer, Jacksonville, Florida Coordinated strategic planning, capital budgeting, project implementation and financial analysis for wood products production facilities in the Southeast.

1979 - $1981 \quad$ Project Engineer, Jacksonville, Florida. Supervised capital project justification and implementation for two wood products facilities in Georgia.

1975 - 1979 _ Process Control Engineer, West Nyack, New York. Managed research projects and process studies - developed quality systems for lumber drying and finger-jointed lumber.

\section{Honors and Fellowships}

US Regular Army ROTC Scholarship, 1968-1969

Certified Quality Engineer

Certified Quality Auditor

\section{Publications}

Noffsinger, J.R. and R.B. Anderson. 2002 Effect of Autocorrelated Data on Composite Panel Production

Monitoring and Control: A Comparison of SPC Techniques. Forest Prod. J. 52(3): 60-67

Noffsinger, J.R. 1979. St Regis Sawmill Scanners - Applications and Accuracy. Electronics in The

Sawmill. Miller Freeman Publications. San Francisco, CA

Noffsinger J.R. and J Ward. 1978 The Effect of Steaming on Drying Rate of Eastern Hemlock Sinker

Heartwood. Report Serial No. 0305-78. St Regis Technical Center. West Nyack. New York.

Noffsinger, J.R. 1978. Log Scanner Evaluation. Report Serial No. 015-78. St Regis Technical Center. West Nyack. New York.

Noffsinger, J.R. and D.D. Huson. 1978. Analyzing Lumber Degrade Due to Drying and Establishing Parameters to Reduce Lumber Degrade. Report Serial No. 005-78. St Regis Technical Center. West Nyack. New York.

Noffsinger, J.R. 1977. Evaluation of Radio Frequency Lumber Moisture Detectors. Report Serial No. 03076. St Regis Technical Center. West Nyack. New York. 
Noffsinger, J.R. 1976. Evaluation of Dry Kiln Performance. Report Serial No. 016-76. St Regis Technical Center. West Nyack. New York.

Noffsinger, J.R. 1976. Kiln Drying Eastern Hemlock Studs II. Report Serial No. 015-76. St Regis Technical Center. West Nyack. New York.

Noffsinger, J.R. 1976. Continuously Rising Temperature (CRT) Drying Process and Some Investigations Into Its Use for Drying Eastern Hemlock Studs. FPRS News Digest, File: G1.12.

Noffsinger, J.R. 1976. Kiln Drying Eastern Hemlock Studs. Report Serial No. 005-76. St Regis Technical Center. West Nyack. New York.

Noffsinger, J.R. 1976. Utilization of Black Cherry Bark in Extruded Particleboard. Master of Science Thesis.

\section{Papers Presented at Conferences}

Hardwood and OSB FPS Hardwood Utilization Conference, Harrisburg, PA. 1997

Analyzing Lumber Degrade Caused by Drying \& Establishing Parameters to Reduce It. Recent Advances in Spruce-Fir Utilization Technology. University of Maine. 1983

Spruce Stud Drying FPS Northeast Section Meeting, Durham, NH 1982

St Regis Sawmill Scanners. Electronic Workshop Sawmill and Plywood Clinic. Portland, OR. 1979

The Effect of Steaming on the Drying Rate of Eastern Hemlock Sinker Heartwood. Spring Meeting of FPRS

Northeast Section and New England Kiln Drying Association. 1976.

Continuously Rising Temperature (CRT) Drying Process and Some Investigations Into Its Use for Drying

Easterm Hemlock Studs. Spring Meeting of New England Kiln Drying Association. 1976.

Statistics

Statistical Process Control

Statistics

Statistical Process Control

\section{Teaching Experiences}

Teambuilding

Conflict Management / Interpersonal Skills

\section{Teaching Interests}

Manufacturing Processes, Forest Products

Quality Management Systems

\section{Research Skills}

Minitab Statistical software for Design of Experiments and Process Control and Analysis

Statgraphics for Response Surface Analysis

SIMCA for Multivariate Data Analysis

\section{Research Interests}

Process Improvement studies in engineered wood products using statistical process control Drying Improvement studies in solid wood

Product Development in wood products

\section{Professional Memberships}

Forest Products Society - Past chairman Drying \& Quality Technical Committees Society of Wood Science and Technology - Past Board Member American Society for Quality - Certified Quality Engineer and Certified Auditor Beta Gamma Sigma (Business Honorary) Gamma Sigma Delts (Agriculture Honorary) 